Florida International University

FIU Digital Commons

FIU Electronic Theses and Dissertations

University Graduate School

$11-14-2017$

\title{
Examining the Relationships between Gender Role Congruity, Identity, and the Choice to Persist for Women in Undergraduate Physics Majors
}

Bronwen Bares Pelaez

Florida International University, baresb@fiu.edu

DOI: 10.25148 /etd.FIDC004054

Follow this and additional works at: https://digitalcommons.fiu.edu/etd

Part of the Adult and Continuing Education Administration Commons, Gender Equity in Education Commons, Higher Education Commons, Higher Education Administration Commons,

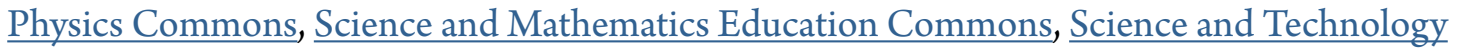
$\underline{\text { Studies Commons, Student Counseling and Personnel Services Commons, and the Women's Studies }}$ Commons

\section{Recommended Citation}

Pelaez, Bronwen Bares, "Examining the Relationships between Gender Role Congruity, Identity, and the Choice to Persist for Women in Undergraduate Physics Majors" (2017). FIU Electronic Theses and Dissertations. 3509.

https://digitalcommons.fiu.edu/etd/3509 


\section{FLORIDA INTERNATIONAL UNIVERSITY}

Miami, Florida

\section{EXAMINING THE RELATIONSHIPS BETWEEN GENDER ROLE CONGRUITY, IDENTITY, AND THE CHOICE TO PERSIST FOR WOMEN IN UNDERGRADUATE PHYSICS MAJORS}

A dissertation submitted in partial fulfillment of the requirements for the degree of DOCTOR OF PHILOSOPHY

in

HIGHER EDUCATION

by

Bronwen Bares Pelaez 
To: Dean Michael R. Heithaus

College of Arts, Sciences and Education

This dissertation, written by Bronwen Bares Pelaez, and entitled Examining the Relationships between Gender Role Congruity, Identity, and the Choice to Persist for Women in Undergraduate Physics Majors, having been approved in respect to style and intellectual content, is referred to you for judgment.

We have read this dissertation and recommend that it be approved.

$\begin{array}{r}\hline \text { Asia Eaton } \\ \hline \text { Norma M. Goonen } \\ \hline \text { Thomas G. Reio, Jr. } \\ \hline \text { Zahra Hazari, Major Professor }\end{array}$

Date of Defense: November 14, 2017

The dissertation of Bronwen Bares Pelaez is approved.

Dean Michael R. Heithaus College of Arts, Sciences and Education

Andrés G. Gil Vice President for Research and Economic Development and Dean of the University Graduate School

Florida International University, 2017 
C Copyright 2017 by Bronwen Bares Pelaez

All rights reserved. 


\section{DEDICATION}

To my sister, Alanna. You have shown me what strength is and how to dig

deeper than you or anyone else expects you to; especially when neither you, nor anyone else thinks you can. I love you.

And to all the women who lead, near and far, keep forging new pathways! 


\section{ACKNOWLEDGMENTS}

Foremost I would like to thank my committee of incredible advisors, Dr. Asia Eaton, Dr. Norma M. Goonen, Dr. Thomas G. Reio, and Dr. Zahra Hazari. I am deeply and especially grateful to Dr. Hazari whose consistent guidance, detailed feedback, cheerleading, and mentorship gave me the confidence to keep moving forward. I have enjoyed our conversations through the years, and appreciate how much I have learned about research from you. Thank you, Zahra!

I am also thankful to many other faculty who have been important to my education at this level: Dr. Linda Bliss, Dr. Haiying Long, Dr. Joy Blanchard, Dr. Bob Farrell (who is deeply missed), Dr. Leonard Bliss, Dr. Vrushali Patil and Dr. Dawn Addy. And for your incredible support, mentorship, and guidance inside the classroom and many times over outside the classroom, Dr. Cathy Akens, "thank you" cannot adequately capture how grateful I am to you for giving me guidance and time in abundance dedicated to this endeavor.

To my mentor and friend, Dr. Suzanne Onorato, who completed this degree while also serving as Director for the Women's Center at FIU. This reminded me every day that this journey I had chosen to embark on was possible; your strength and passion for college student development, safety, and success will never stop serving as a source of aspiration and inspiration for me. Thank you for all of your personal and professional support through the years.

To my mini-cohort: Nashira Williams, Sabrena O’Keefe, Michelle Castro, Yinhong Duan, Amanda Niguidula, and many others. Sharing various parts of this journey made this lonely road full of laughter, learning, and support. 
To the participants in this study who shared their stories with me, thank you. These women humbled and inspired me as I grew into this privileged position as "researcher."

Many thanks to my Women's Center staff, students, and colleagues near and far. And a special thanks to Dr. Beverly Dalrymple: When I dreamt of my career as an M.Ed. student, I wished for a job that would be fulfilling in every way; thank you for giving me the opportunity to realize that dream at FIU. And thank you for reminding me that this dissertation was an "exercise in research" and I should approach it as I would any other project; one step at a time.

I cannot thank my friends and extended family enough for their support and patience. I have sacrificed attention to, and time with, all of you the last few years while I was working on my dissertation. I cannot thank you enough for you being there for me, for rooting for me, and for listening to my boring stories about life as a doctoral student; I tried to make them as funny as possible to make up for having little else to talk about.

To my best friend, my husband, my love; Robert, you have been supportive and generous all along, no matter what. Thank you for letting me convince you to complete your MBA just so that we could "suffer" together; I'm so proud of you for accepting that challenge, and for finishing it with great success. Most importantly, we've never stopped having fun together, even when we didn't have many reasons to. I couldn't ask for a more perfect partner.

And thank you to my brother, Garrett. Your unconditional love and sense of humor always puts a smile on my face and helps me keep perspective in this life. Love you, GoGo! 
Finally, and most importantly, to my parents, John and Diane, thank you will never be enough. You instilled in me a deep value for education, hard work, and excellence; all of which have served as my compass throughout this process. Everything I am and everything I have, I owe to you. I love you!

All of you, and so many others along the way, have made this accomplishment possible and for that I am grateful. 


\title{
ABSTRACT OF THE DISSERTATION \\ EXAMINING THE RELATIONSHIPS BETWEEN GENDER ROLE CONGRUITY, IDENTITY, AND THE CHOICE TO PERSIST FOR WOMEN IN UNDERGRADUATE PHYSICS MAJORS
}

\author{
by \\ Bronwen Bares Pelaez \\ Florida International University, 2017 \\ Miami, Florida \\ Professor Zahra Hazari, Major Professor
}

Persistent gender disparity limits the available contributors to advancing some science, technology, engineering, and math (STEM) fields. While higher education can be an influential time-point for ensuring adequate participation, many physics programs across the U.S. have few women in classroom or lab settings. Prior research indicates that these women face considerable barriers. For university students, faculty, and administration to appropriately address these issues, it is important to understand the experiences of women as they navigate male-dominated STEM fields.

This explanatory sequential mixed methods study explored undergraduate female physics majors' experiences with their male-dominated academic and research spaces in the U.S. The conceptual framework consisted of physics identity, gender role congruity, assumptions about the "ideal" scientist, and self-reported plans to persist in the field (measured by bachelor's degree completion, graduate school plans, and physics-related career plans). Utilizing the American Physical Society (APS) 2016 Conferences for Undergraduate Women in Physics (CUWiP) pre-conference survey data, responses from 
900 females were analyzed using regressions followed by 18 semi-structured interviews with CUWiP sample participants.

Physics identity was highly predictive of participants' self-reported persistence plans. A factor analysis revealed that gender role congruity is comprised of three distinct social roles: extrinsic agentic (e.g., power, financial rewards, status), intrinsic agentic (e.g., self-direction, demonstrating skills, independence), and communal (e.g., working with people, helping others). Intrinsic agency was highly correlated with physics identity and long-term persistence (graduate school and career), and communal roles were negatively correlated with students' short-term persistence (undergraduate physics degree completion). Extrinsic agency was correlated with neither identity nor persistence.

The 18 interviews were phenomenographically analyzed revealing that participants experience relationships with the conceptual framework in five qualitatively different ways, called categories of experience. These categories are: The Assured, The Solitary, The Communal, The Reflective, and The Ambassadors. The categories elaborate on the quantitative results by providing nuanced explanations of how women negotiate aspects of their gender identity related to the conceptual framework.

The results provide a broad vantage point of women's experiences as physics majors which may aid university faculty and administration with gender equity goals for physics and other male-dominated STEM fields. 


\section{TABLE OF CONTENTS}

$\begin{array}{ll}\text { CHAPTER } & \text { PAGE }\end{array}$

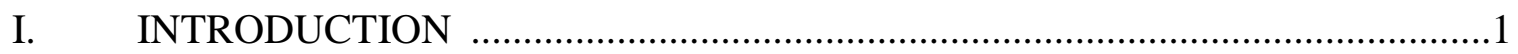

Background of the Problem …………………….........................................

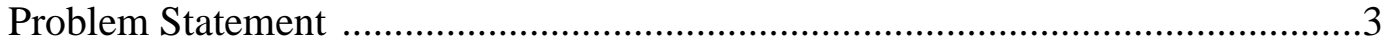

Purpose of the Study ......................................................................................

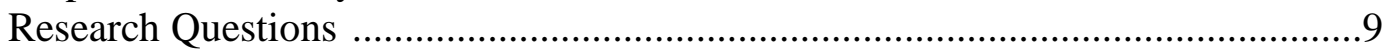

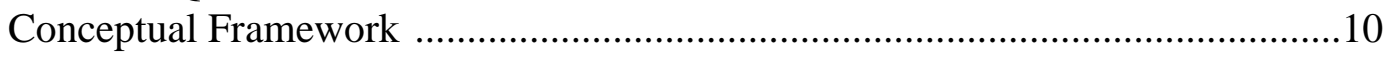

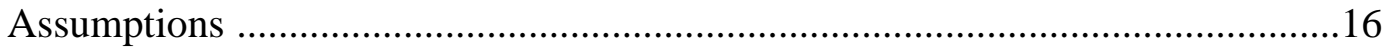

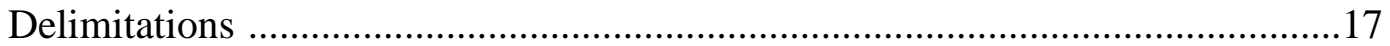

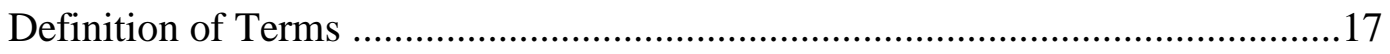

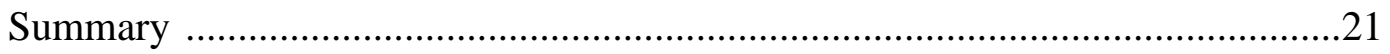

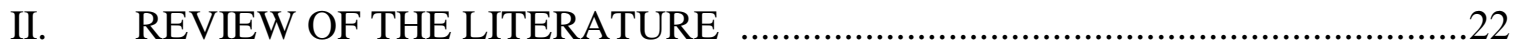

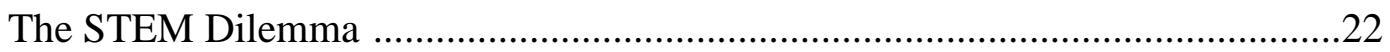

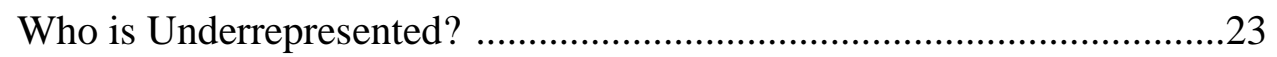

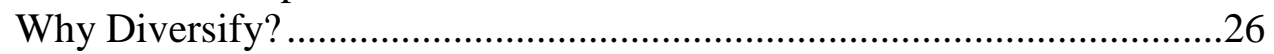

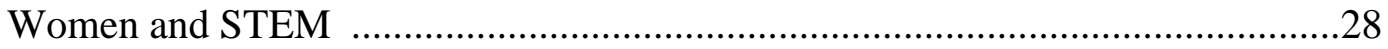

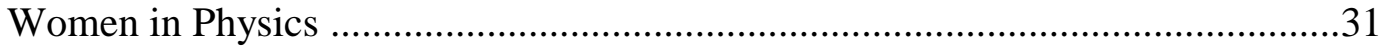

Situating the Conceptual Framework ………………...........................................32

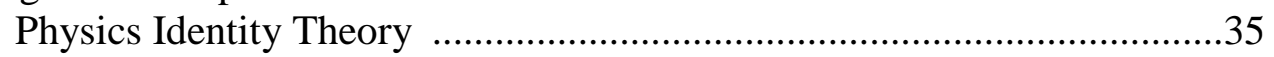

Gender Role Congruity Theory ………………………….......................40

Ideal Scientist Concept ………………………………….....................43

Women's Persistence on the STEM Pathway ………………………….....47

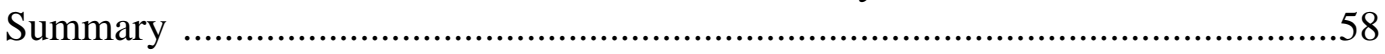

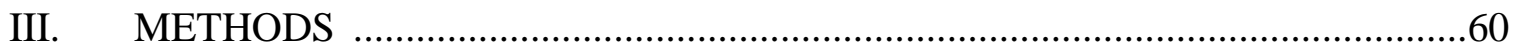

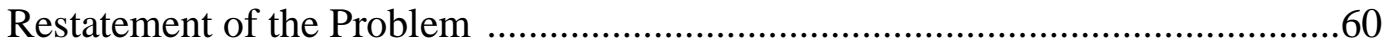

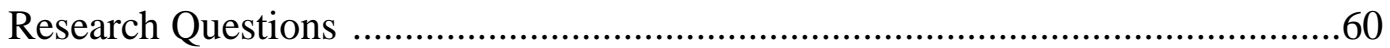

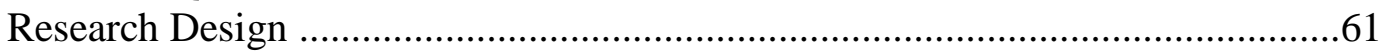

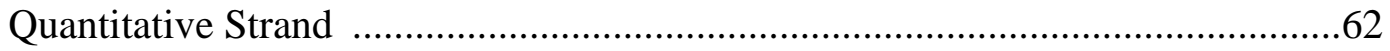

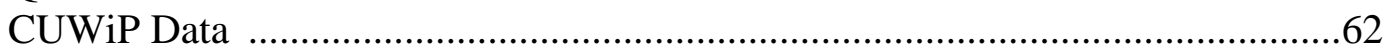

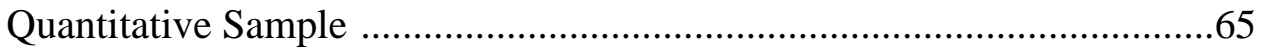

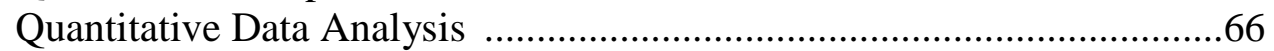

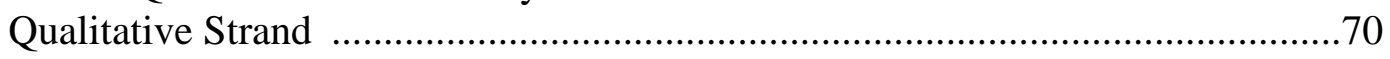

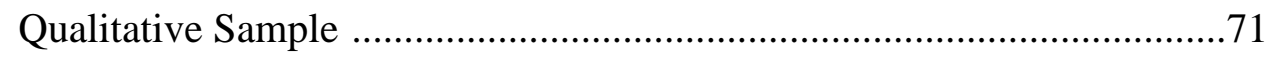

Qualitative Data Collection …………………………………….........76

Qualitative Data Analysis .....................................................................77

Reliability and Reflexivity .................................................................. 81

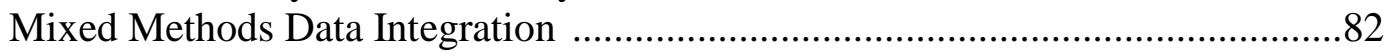

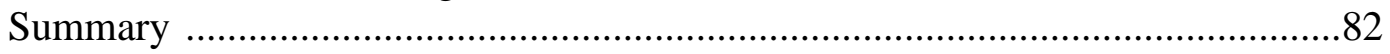




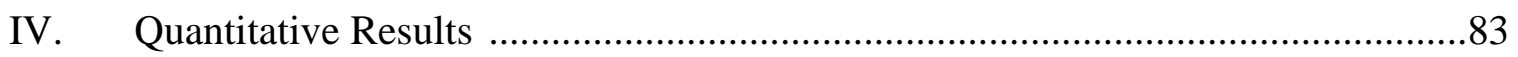

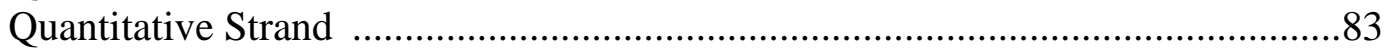

Sample Population Demographics .................................................................

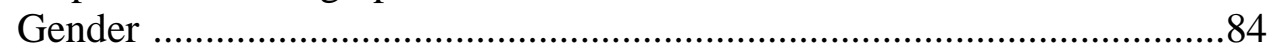

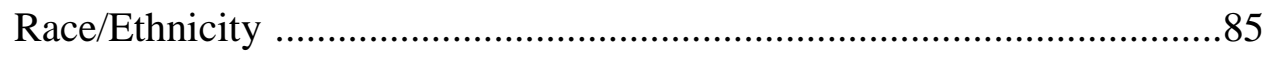

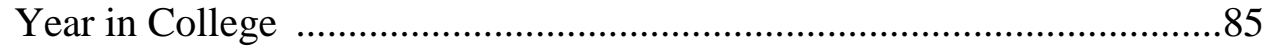

Physics Courses Taken ......................................................................86

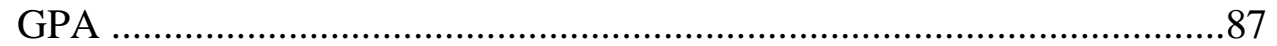

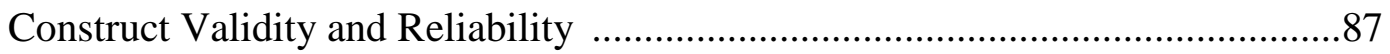

Gender Role Congruity .........................................................................8

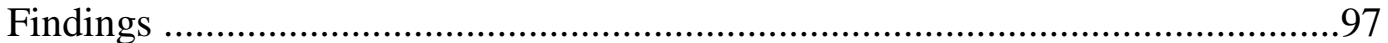

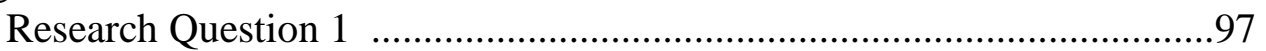

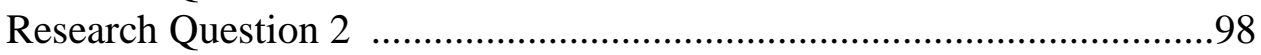

Research Question 3 ....................................................................100

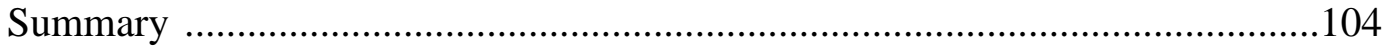

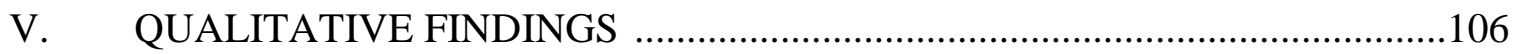

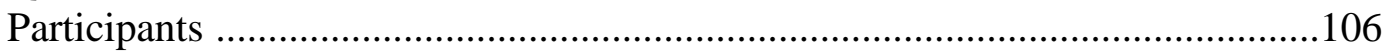

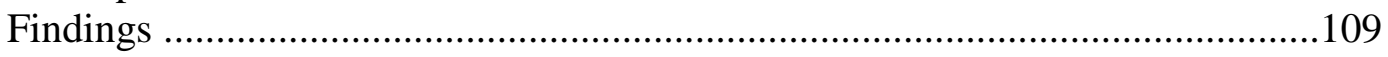

Outcome Space Structure ………………………................................110

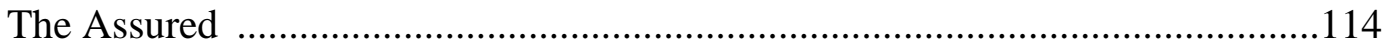

Identity Congruence ……………………………………………....114

Ideal Congruence …………………………………....................119

Gender Congruence ……………………………….....................121

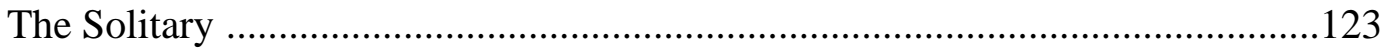

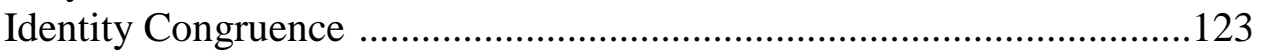

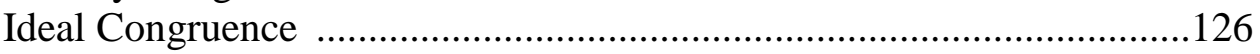

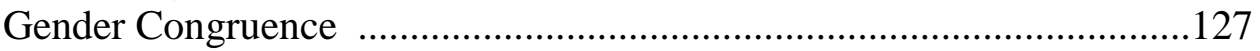

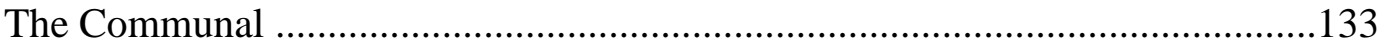

Identity Incongruence ………………………………...................135

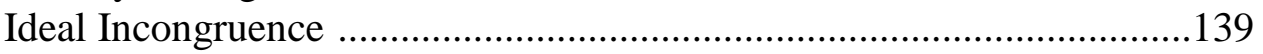

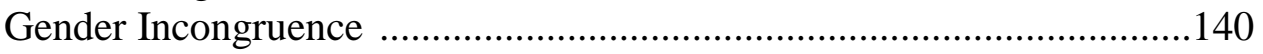

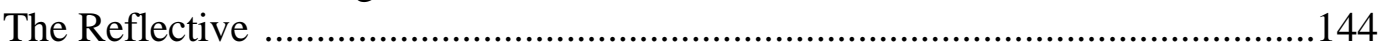

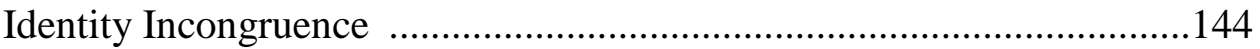

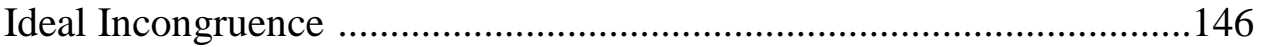

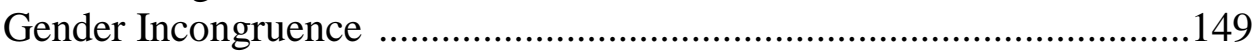

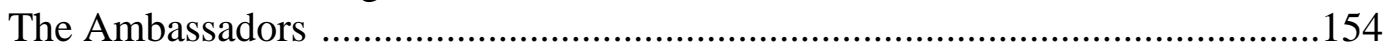

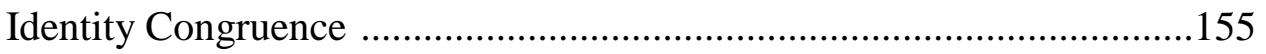

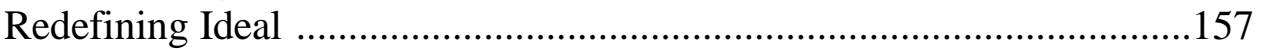

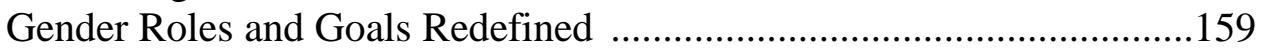

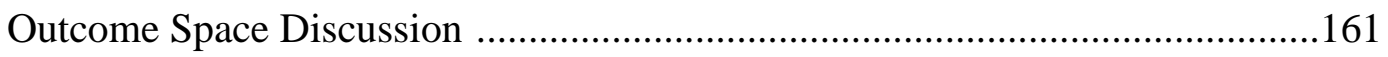

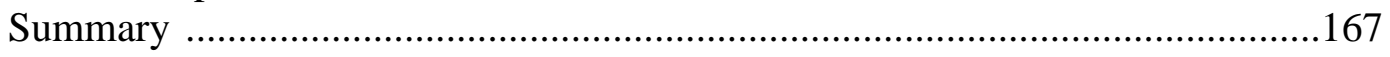




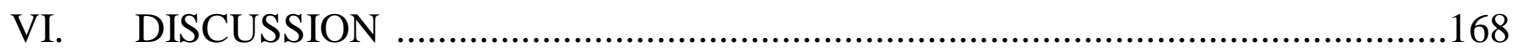

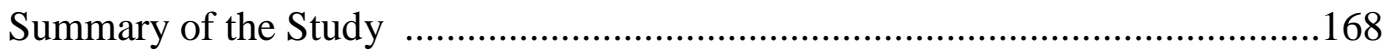

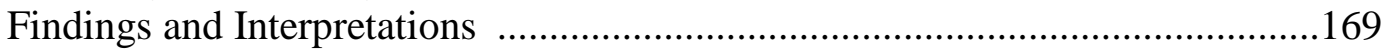

Response to Research Questions …………………..........................................169

Research Question 1 .....................................................................170

Research Question 2 …………………...........................................170

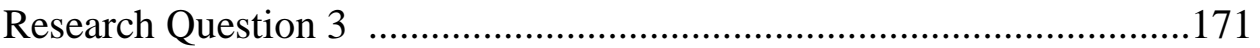

Research Questions 4 and 5 ...............................................................173

Explanatory Sequential Mixed Methods Analysis and Interpretation .................178

Research Question 1 .................................................................178

Research Question 2 ………………………………...................181

Research Question 3 ....................................................................187

Mixed Methods Discussion Summary ..............................................................194

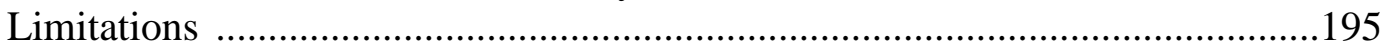

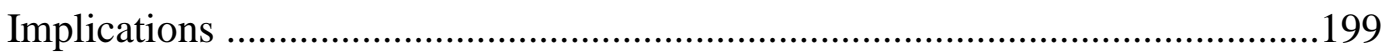

Theoretical Implications .................................................................199

Implications for Faculty and Academic Programs .................................200

Implications for University Administrators ………………..................202

Recommendations for Future Research .....................................................204

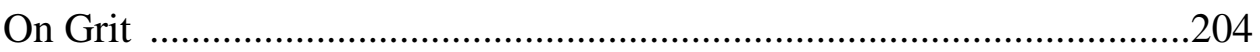

Incorporating Male and Non-Binary Gender Perspectives ......................205

Highly Communal/Highly Agentic Success in STEM ..........................206

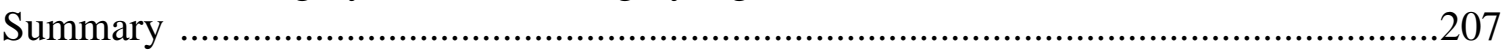

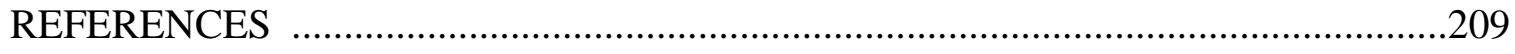

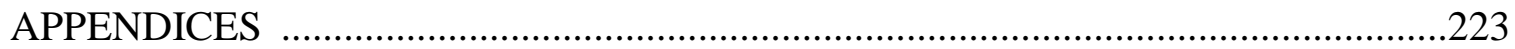

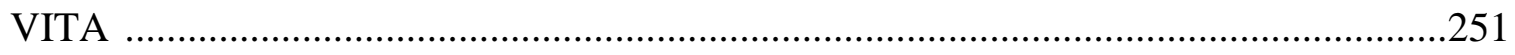




\section{LIST OF TABLES}

TABLE

PAGE

1. Qualitative Strand Participants' CUWiP Survey Scores .............................................70

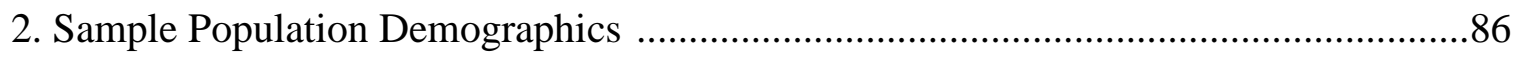

3. Gender Role Congruity Construct Validity ……………............................................90

4. Gender Role Congruity Descriptive Statistics .........................................................91

5. Factor Analysis of Physics Identity for Self …………..............................................

6. Factor Analysis of Ideal Physics Student ………………..........................................96

7. Physics Identity and the Ideal Physics Student Descriptive Statistics ...........................96

8. Perceptions of the Ideal Science Student as Predictor of Physics Identity ....................97

9. Gender Goals as Individual Predictors of Physics Identity ..........................................98

10. Linear Regression for Gender Goals and Physics Identity .......................................99

11. Persistence Self-reported as Academic and Career Plans Descriptive Statistics ......101

12. Linear Regression for Physics Identity, the Ideal Physics Student, and Gender Goals as Predictors of Self-Reported Bachelor's Degree Persistence ......................................101

13. Linear Regression for Physics Identity, the Ideal Physics Student, and Gender Goals as Predictors of Self-Reported Graduate Degree Persistence

14. Linear Regression for Physics Identity, the Ideal Physics Student, and Gender Goals as Predictors of Self-Reported Career Plans as Persistence 


\section{LIST OF FIGURES}

FIGURE

PAGE

1. Procedural diagram for explanatory design of the mixed methods study

2. Procedural diagram for the quantitative strand of the study 16

3. Framework for students' identification with physics

4. Resulting goal-endorsement factors for agentic and communal goals

5. Gender roles/goals congruity CUWiP survey question

6. Scores and corresponding tags associated with participants' responses to the CUWiP survey questions related to the conceptual framework of this study

7. Overview of the code system as displayed in MAXQDA .......................................78

8. Histogram of the number of physics courses completed by CUWiP respondents $\ldots . . . .87$

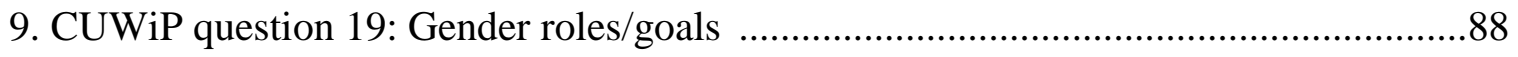

10. Scree test plot of gender role congruity variables ...........................................89

11. CUWiP pre-conference survey question 5: Identity and ideal ...............................92

12. CUWiP pre-conference survey question 6: Identity and ideal ..............................93

13. CUWiP pre-conference survey questions 1, 2, and 3: Persistence ..........................100

14. Map of approximate geographic location of qualitative participants' undergraduate university 


\section{CHAPTER I}

\section{INTRODUCTION}

One of the things that I really strongly believe in is that we need to have more girls interested in math, science, and engineering. We've got half the population that is way underrepresented in those fields and that means that we've got a whole bunch of talent...not being encouraged the way they need to.

- President Barack Obama, February 2013 (The White House Office of Science and Technology Policy, para. 1)

The most important thing I learned is that a scientist can look just like me.

- Mykel Sisk, NexGeneGirls Intern, Boys \& Girls Clubs of San Francisco

(Gardiner, 2013, homepage image)

Common themes across the research on women in male-dominated fields of work and study in higher education, specifically the areas of science, technology, engineering, and mathematics (STEM), indicate both internal and external barriers that contribute to female students enrolling and persisting at significantly lower rates than their male counterparts. Chapter I will cover the background of the problem, problem statement, purpose of the study, research questions, conceptual framework, assumptions, limitations, and definitions of terms for this proposed study.

\section{Background of the Problem}

Much of the literature references Hall and Sandler's (1982) “chilly climate" report on women's experiences in STEM fields and in the classroom (Allan \& Madden, 2003; Fassinger \& Asay, 2006; Salter, 2003; Sandler, Silverberg, \& Hall, 1996). In 2003, Urry wrote, "in physics departments around the country, women are feeling ill at ease, out of place, and not at home" (p. 12). Jorgenson (2002) also reported evidence that women who do persist in these fields are sometimes unaware of their own gender identity and do 
not see themselves as pioneers, thereby experiencing a "chilly climate" unconsciously, if at all.

Despite lower rates of enrollment and persistence in STEM fields and complex challenges faced in the classrooms and along the career paths of these fields, women are earning more bachelor's degrees in a number of STEM areas than in the past (Hazari, Tai, \& Sadler, 2007; Hughes, 2010; National Science Foundation, 2014). The 2015 National Science Foundation (NSF) report produced in collaboration with the National Center for Science and Engineering Statistics and the Directorate for Social, Behavioral, and Economic Sciences, as a compliance response to the Science and Engineering Equal Opportunities Act (Public Law 96-516) entitled, Women, Minorities, and Persons with Disabilities in Science and Engineering, outlined an overview of the current statistics on who is participating in STEM education and work in the U.S. The report presents the current status of women in what they refer to as science and engineering (S\&E) fields:

The representation of certain groups of people in science and engineering (S\&E) education and employment differs from their representation in the U.S. population. Women, persons with disabilities, and three racial and ethnic groups-blacks, Hispanics, and American Indians or Alaska Natives-are considered underrepresented in S\&E...In most fields, the proportion of degrees awarded to women has risen since 1993. The proportion of women is lowest in engineering, computer sciences, and physics. (pp. 2 \& 5)

There are a number of arguments available for why and how increasing women in STEM fields will benefit the fields which include the positive impacts of diversity, inclusivity and more robust dialogue on intersectionality (Jones \& Wijeyesinghe, 2011). Additionally, a generalized belief exists that if a field is missing vast portions of the population as active participants, it runs the risk of also missing half of the innovation 
and leadership that will usher the field toward the future (Cheryan, Master, \& Meltzoff, 2015; Hazari et al., 2007; Hendley \& Charles, 2015; Kenway \& Gough, 1998; Oh \& Lewis, 2011). There is no clear consensus among researchers regarding the sources, nor the solutions, to the complex factors contributing to low recruitment and retention rates for women in STEM fields of study and work, only pieces of the puzzle and suggestions. Many researchers contextualize the complex nature of gender disparity in the American higher education landscape because an increasing number of institutions have stated publicly that they seek to increase the number of women and minorities graduating from their STEM programs (Dyer, 2004; Espinosa, 2011; Gonsalves, 2014; Griffith, 2010; Hazari, Sadler, \& Sonnert, 2013; Johnson, 2012; McCarthy \& Wolfe, 1975; Peng \& Jaffe, 1979; Perna et al., 2009; Sader, 2011; Sax, 1994). According to researchers, there are a series of root issues that contribute to the complex nature of this disparity.

\section{Problem Statement}

Some of the root issues that contribute to gender inequity in STEM fields are imbedded within the culture and bureaucracy of higher education institutions manifesting in examples such as faculty demographics, hiring and promotion practices, family leave practices, a common lack of role models with whom a diverse student body can identify, and discriminatory practices both within and outside of the classroom (Ceci \& Williams, 2011; Cole \& Espinoza, 2014; Espinosa, 2011; Griffith, 2010; Hendley \& Charles, 2015; Johnson, 2007).

Other root issues are located within the STEM fields themselves can be found within the language used to describe those fields that lead people to assume that these fields are themselves prescriptively masculine (Bystydzienski \& Brown, 2012; Faulkner, 
2001, 2007; Johnson, 2014), or that these fields require raw intellectual talent, and women stereotypically are thought not to possess raw talent (Leslie, Cimpian, Meyer, \& Freeland, 2015; Shanahan \& Nieswandt, 2010). Furthermore, these assumptions are reinforced by other levels of education and by those outside of these fields. Bian, Leslie, and Cimpian (2017) reported that children as young as 6 years of age endorse these assumptions and are willing to assign boys as those who are more capable of taking on tasks that require high-level intellectual ability. Furthermore, the interests of children at this age are influenced by the gendered stereotypes.

Still, other root issues persist in broader societal perceptions of gender-science stereotypes such as the media representations of scientists and engineers far beyond the classroom (Cheryan et al., 2015; Miller, Eagly, \& Linn, 2015; Moulton Belec, 2015; Rosenbaum, 2013). Many people are involved in socializing children into the gender roles that match their sex, and children receive messages about what their role will be throughout their lifetime including whether or not a STEM pathway is an appropriate goal for them to set (Bem, 1981; Bystydzienski \& Brown, 2012; Carlone, 2004; Rahm, 2007). Therefore, gender role congruity, a theory that individuals are socialized into roles that are prescriptively male (i.e., agentic, having the capacity for power and responsibility over one's own life), and roles that are prescriptively female (i.e., communal, which is to be oriented toward roles and characteristics that are traditionally other-oriented; Diekman \& Eagly, 2000; Diekman \& Goodfriend, 2006; Eagly \& Diekman, 2000; Eagly \& Karau, 2002), has been applied to examine which STEM fields are widely accepted as agentic or communal thereby explaining why some fields of study have made progress toward gender equity while others have not (Diekman, Brown, 
Johnston, \& Clark, 2010). The STEM fields that are perceived as offering communal opportunities experience gender equality across undergraduate and graduate programs (e.g., medicine, biology, dentistry, zoology, etc.; Diekman et al., 2010; Diekman, Clark, Johnston, Brown, \& Steinberg, 2011; NSF, 2015). Conversely, STEM fields that are perceived to be agentic experience persistent gender inequality across undergraduate and graduate programs (e.g., physical sciences, engineering, computer sciences, economics, etc.; Diekman et al., 2010; Diekman et al., 2011; NSF, 2015). The organizational structure of roles assigned by gender-science stereotypes connects directly with the language and development of male-dominated STEM fields still present today (Diekman et al., 2010; Miller, Eagly, \& Linn, 2015).

Recent, publicity-surrounded examples of the roots of these issues include statements made by former Harvard President, Lawrence Summers, regarding the biological differences that result in "men outperforming women in maths and sciences," and his assertion that barriers or discrimination for female academics is no longer an issue (Goldenberg, 2005, para.1). These sentiments have been reiterated as recently as August 2017 when an internally circulated "manifesto" entitled, Google’s Ideological Echo Chamber, written by a senior software engineer at Google went viral; in the memo, the male author posited that the company does not need "programs to recruit racial minorities" and women because women are "less well suited for engineering work" (Barman, 2017, para.1), and that "We need to stop assuming that gender gaps imply sexism" (Conger, 2017, para.2).

In 2015, statements made at an international conference for science journalists in South Korea by Nobel Prize winning scientist, Tim Hunt, went viral after he publicly 
detailed his ideas on the "trouble with girls" stating: "Three things happen when they are in the lab, you fall in love with them, they fall in love with you, and when you criticize them they cry" (BBC News, 2015, para. 7). Still more recent examples demonstrating a broken accountability system for gender discrimination in the sciences include three high profile sexual harassment cases in the field of astronomy (Feltman, 2016). These cases became high profile in response to an initial case that went viral in October, 2015 when BuzzFeed journalist, Azeen Ghorayshi covered the sexual harassment investigation against Nobel laureate contender and astronomer, Geoff Marcy. The sanctions he faced as a result include clearly defined behavior expectations and a warning that another report of this nature will result in his suspension or firing. Ghorayshi interviewed a wellestablished astronomy professor from Harvard, David Charbonneau, to better relay the implications of this case:

"Geoff Marcy is undeniably the most prominent exoplanet researcher in the U.S." he said, referring to the study of planets beyond our solar system. "The stakes here couldn't be higher. We are working so hard to have gender parity in this field, and when the most prominent person is a routine harasser, it threatens a major objective nationally." (Ghorayshi, 2015)

With these $21^{\text {st }}$ century examples of prominent men who feel comfortable using their positions of power, both behind closed doors and in public, to position the place and competence of women in STEM fields as inferior, the issue of a "chilly climate" culture within academic programs and within classrooms across the country is considered an issue worthy of continued attention. 


\section{Purpose of the Study}

The present study focused on undergraduate women majoring in a maledominated STEM area of study, specifically the physical sciences, their physics identity development, their perceptions of the "ideal" scientist, how they understand gender roles within their chosen field, and how they use this information to inform their decisions to persist in their major. Undergraduate students can elect to take certain science classes beyond related requirements of secondary and higher education, and many students remove themselves from STEM pathways as they enter college (Clark Blickenstaff, 2005; Chen, 2009; Griffith, 2010; Sadler et al., 2012; Shapiro \& Sax, 2011). Therefore, I wanted to learn more about how women make these decisions to major in maledominated science subjects such as physics in college (NSF, 2015), how female students navigate these spaces, and how they think about their decisions about persisting in their major and make future education and career plans.

The dissertation utilized an explanatory sequential mixed methods approach, following frameworks provided by Tashakkori and Teddlie $(1998,2003)$ and Creswell $(2014,2015)$. The research design involved collecting and analyzing quantitative data first, followed by a second phase during which qualitative data was collected and analyzed, and finally a phase during which the qualitative findings were used to further explain the quantitative findings. Utilizing the qualitative data to explain how the variables tested interact served as a source of strength for this design.

In the first quantitative phase, or strand, of the study, survey data were collected by the American Physical Society (APS) from 953 college students who registered to attend the 2016 Conferences for Undergraduate Women in Physics (CUWiP), which is an 
annual conference held on the same weekend at nine locations nationally. The male and gender non-binary respondents were removed from the sample leaving 900 female respondents in the dataset that was analyzed. These data were analyzed to test the correlational factors of women undergraduates' physics identity, their perceptions of the "ideal" scientist, their perceptions of gender role congruity in their STEM field, and their plans to persist in their chosen science major.

The second qualitative strand of the study was conducted as a follow up on the quantitative results to help further explain them. In this exploratory follow-up, semistructured interviews were conducted to explore perceptions of gender role congruity and gender equality in their chosen science major with purposefully selected CUWiP participants who exemplified specified demographic criteria, extreme or outlier cases, and significant relationships between the four variables making up the conceptual framework for this study. Furthermore, I gathered qualitative data about how these students understand the impact of these factors on their plans to persist in their chosen field. Figure 1 outlines the steps of this mixed methods study: 


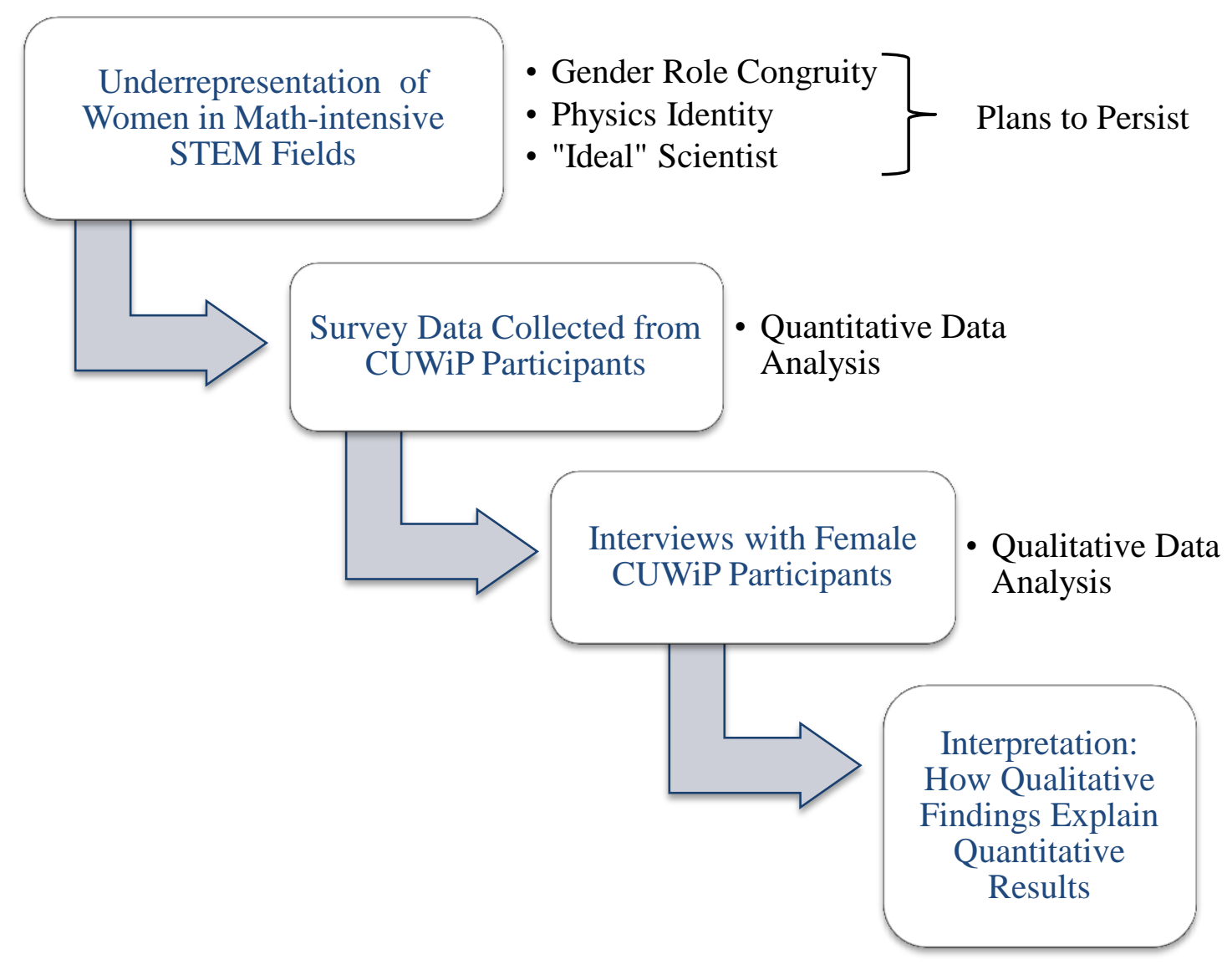

Figure 1. Procedural diagram for explanatory design of the mixed methods study. Chapter 3 will outline these steps with a detailed description of the methods and phases employed for this study.

\section{Research Questions}

This study is framed by five research questions, three of which were explored during the quantitative strand and two of which were explored during the qualitative strand. Particularly in the qualitative strand and mixed methods discussion, the qualitative data collection and analysis was used to integrate understanding across both 
strands. All of these questions were responded to by the participant population under investigation for this study. The research questions are as follows:

1. Quantitative: Is the way undergraduate women in physics see the "ideal" physics student related to their physics identity?

2. Quantitative: How does gender role congruity relate to physics identity?

3. Quantitative: What factors (i.e., physics identity, gender role congruity, perceptions of the "ideal" science student) relate to plans to persist?

4. Qualitative: How do the experiences of undergraduate women in physics majors delineate their perspectives related to their physics identity, the "ideal" physics identity, and gender role congruity within their chosen field of study?

5. Qualitative: How are the experiences of the participants similar, or different, based on the theoretical framework that guided this study?

\section{Conceptual Framework}

For the purposes of this study, the conceptual framework consisted of the following four pieces: Physics Identity Theory (Hazari et al., 2010), Gender Role Congruity Theory (Diekman et al., 2010; Eagly \& Diekman, 2005), the "Ideal” Scientist concept (Bian et al., 2017; Leslie et al., 2015; Sader, 2011; Shanahan \& Nieswandt, 2010), and persistence (Chang, Eagan, Lin, \& Hurtado, 2011; Espinosa, 2011; Griffith, 2010; Shapiro \& Sax, 2011; Toven-Lindsey, Levis-Fitzgerald, Barber, \& Hasson, 2015). These theories and concepts were chosen because the use of multiple theories and concepts within a study has been well documented in the literature as a means to adequately convey the complexity and contextualization of issues related to underrepresented populations in STEM. Furthermore, this combination of theories and concepts provided new opportunities to advance our understanding of the topic. The approach was well justified by research questions that explored not only these individual 
variables, but also the points at which they intersect, if any, and the relational factors among them.

\section{Physics Identity}

Physics identity serves as a helpful lens when gathering and analyzing data about why some women and minorities persist in this field despite nuanced barriers (Carlone \& Johnson, 2007; Cleaves, 2005) Likewise, physics identity, or a lack of physics identity development, can help researchers understand why many women and minorities opt out (Carlone, 2004; Hazari, Sonnert, Sadler, \& Shanahan, 2010; Hazari et al., 2013; Jones, Howe, \& Rua, 2000; Shanahan, 2007) because a strong link has been found between “physics identity and physical science career choices" (Hazari et al., 2010, p. 994). To better understand how students learn their way into the knowledge and cultures of STEM fields, many researchers find it useful to look at groups of people who are opting out, which in some STEM fields continues to be overrepresented by groups other than White and Asian males (Carlone \& Johnson, 2007; NSF Report, 2014). But physics identity is about more than who opts in and out. Physics identity provides a framework beyond what and how people understand their own learning of physical science concepts; an identity lens also captures the cultural aspects of developing interest in a science field that is great enough to allow underrepresented students to persist despite the barriers (Brickhouse, 2001; Hazari et al., 2010). When looking at women pursuing undergraduate degrees in science, four constructs of a physics identity have been identified: feeling recognized, interest, performance, and competence (Hazari et al., 2010; see also Carlone, 2004). For a student to be willing and able to make plans to persist in their chosen 
physical science field, they must have developed conceptions of all four of these identity constructs. Furthermore, in physics, women (across both majority and under-represented groups) consistently report "lower self-perceptions toward physics" (Hazari et al., 2013), which impacts their willingness to make plans to persist in this field of study. For these reasons, a physics identity framework was critical to the foundation of this study.

\section{Gender Role Congruity}

Gonsalves (2014) and Danielsson (2009) present an argument for another dimension beyond that of identity, specifically in the field of physics, which calls for further exploration of gendered roles within the field. As described by Gotschel (2014), in recent years, "a shift or extension in the research on the gendered culture and image of physics (education) can be noted that stretches from a more static picture of 'having gender' to a more dynamic understanding of 'doing gender' in physics" (West \& Zimmerman, 1987 as cited in Gotschel, p. 532). Both Danielsson's and Gonsalves' research emphasized that in addition to identity, it is important to recognize the "complex negotiation" women must navigate to successfully pursue a career as a physicist. Therefore, in addition to the theoretical framework of a physics identity, Role Congruity Theory (Eagly \& Diekman, 2005) will serve as a complementary lens through which to analyze the data I collected. In their work on how stereotypes and prejudices impact the perceptions of an individual in ways that the individual may or may not have control over, Eagly and Diekman reflect deeply upon, and in some ways challenge, the use of the term prejudice as defined by Gordon Allport as "an antipathy based upon an inflexible generalization" (1979, p. 9). Eagly and Diekman provide dimensions to further understand the complexity, accuracy, and contextualized nature of the application of 
prejudice as a social problem through the advances in sociocultural sciences since

Allport's definition:

[A] member of a group whose stereotypical attributes are thought to facilitate performance in a role is ordinarily preferred over a member of a group whose stereotypical attributes are thought to impede performance, even in the absence of objective differences between the two individuals. Such incongruity between stereotypical characteristics and social roles does not necessarily lead to a generalized hostile attitude toward the mismatched individual but to a decline in evaluation relative to a matched individual in the context of the particular role. (Eagly \& Diekman, 2005, p. 19)

Using these constructs of role congruity expectations for individuals as the premise, both researchers have done extensive work to develop applications of this theory specifically for gender roles in leadership, as well as gendered roles and goals in STEM fields (Diekman \& Goodfriend, 2006; Diekman et al., 2010; Diekman, Clark, Johnston, Brown, \& Steinberg, 2011; Johnston \& Diekman, 2015; Eagly \& Karau, 2002). What Diekman and her colleagues have discovered is that there are specific dimensions of gender commonly accepted at the group level that can create prejudices that impact women's interest and participation in roles and goals that embody the dimensions of what it means to be male, therefore causing these roles to be categorized as best performed by males.

To better understand why people pursue STEM careers, Diekman et al. (2010, 2011) used these dimensions to further define the social roles they theorize to impact persistence toward goals in these fields. The researchers have defined agentic goals as those men have traditionally occupied which focus on, "agency, or self-orientation" and define communal goals as those women have traditionally filled through, "caretaking roles associated with communion, or other-orientation" (Eagly, Wood, \& Diekman, 2000 as cited in Diekman et al., 2010, p. 1052). These gender role dimensions are oriented 
around sources of power; therefore, an individual's ability to be successful in reaching their goals is impacted by whether the roles they pursue as logical steps towards their goals allow the individual to receive and exert power in a manner congruent to their stereotypical gender role assignment. Gotschel (2014) cited the work of Knorr-Cetina (1999) to best capture the ways in which women pursuing physical sciences may be perceived as pursuing roles incongruent by Diekman et al.'s definitions: “[Knorr-Cetina] compared gender practices in molecular biology laboratories and high energy physics communities, and noticed that physicists (with the exception of Italians) exhibited a kind of 'mono-gender' that is closer to masculinity than to femininity" (p. 532).

\section{Ideal Scientist}

Shanahan and Nieswandt (2010) recommend the consideration of an additional perspective when research specific to science education employs student agency and identity variables. Their research shows a fairly consistent social structure element is present in science classrooms regardless of geographic location or instructor. Additionally, they found that students can articulate role understandings among students in science classes, "characterized by references to expectations of intelligence, experimental skill, scientific mindedness, and appropriate classroom behavior" (Shanahan \& Nieswandt, 2010, p. 367). Therefore, the concept of the "ideal" science student was utilized to round out the framework for this study. In addition to serving as the focus of Shanahan and Nieswandt's work, the topic of the "Ideal" Scientist is also a theme identified by Sader in a study of female computer scientists (2011), as well as the focus of the much discussed 2015 article by Leslie, Cimpian, Meyer, and Freeland, Expectations of Brilliance Underlie Gender Distributions Across Academic Disciplines 
and the 2017 article by Bian, Leslie, and Cimpian, Gender Stereotypes about Intellectual Ability Emerge Early and Influence Children's Interests.

In Sader's qualitative study, she found that for women in the male-dominated field of computer science, their gender identity and their STEM identity, could not be mutually exclusive, and thereby "must fit together" (Sader, 2011, p. 125). Therefore, Sader used this finding to make observations about how her participants understood their gender and STEM identity, and to theorize that for a woman to choose to pursue this male-dominated field, she must use the same language to define a successful computer scientist as she does to describe herself. The idea of congruence between an individual's definition of self and her definition of the "ideal" (Sader, 2011) served alongside the Shanahan and Nieswandt defined expectations of the science student role to round out the paradigmatic context of this study.

The three concepts of gender role congruity, physics identity, and the "ideal" scientist were utilized to determine the relationships between these specific constructs, and then examined their relationships with female undergraduate's self-reported plans to persist in a male-dominated STEM field such as physics or engineering. Figure 2 represents these procedures: 


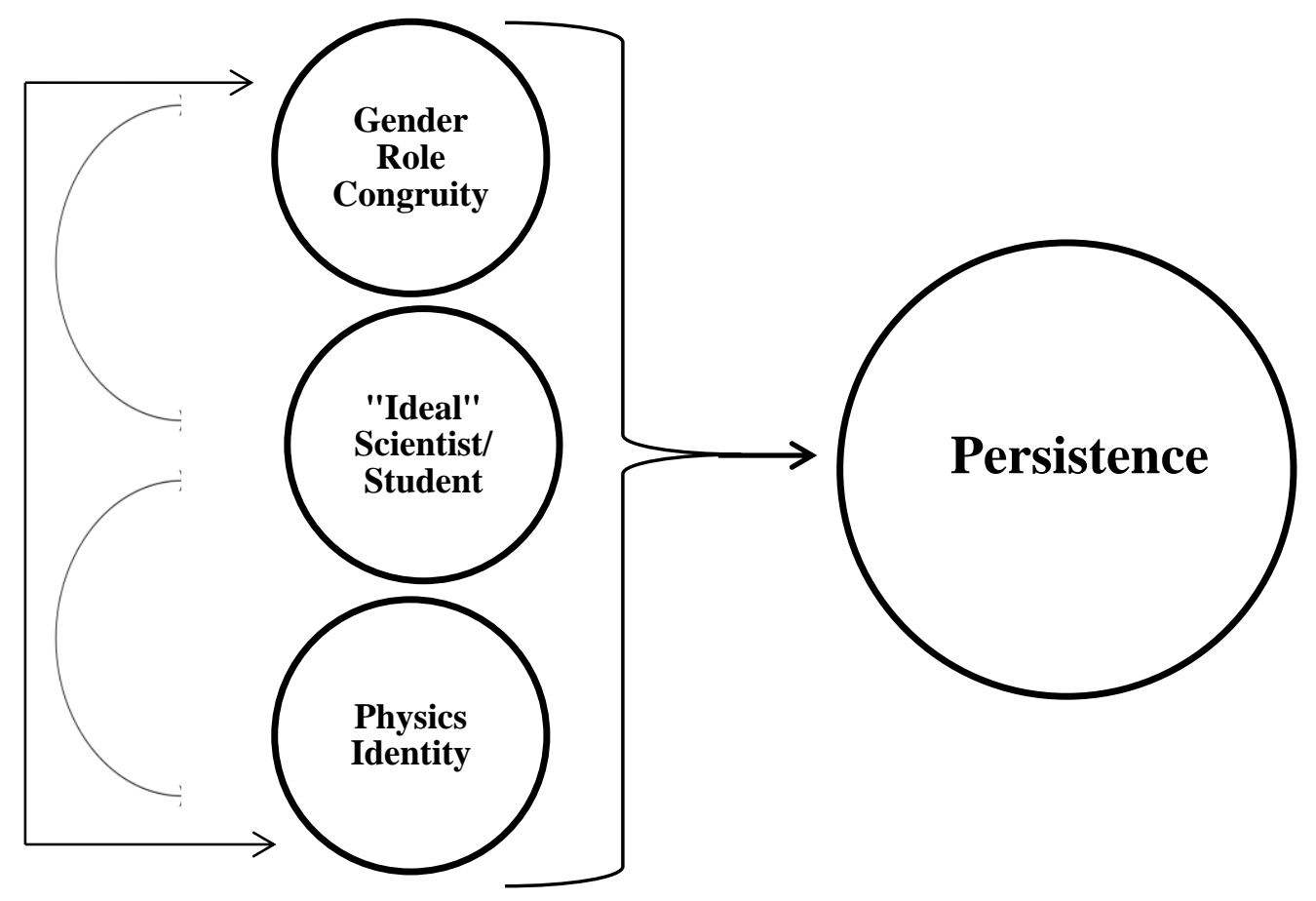

Figure 2. Procedural diagram for the quantitative strand of the study.

\section{Assumptions}

Assumptions are imbedded into all research studies, and this study was no different. One of the assumptions that underpinned this study was that gender equity across all fields of study and professional work, including STEM fields in which women are currently underrepresented, is a valid and appropriate line of inquiry. The welldeveloped and growing body of literature on the topic was utilized to bolster the assumption. Although dissenting voices continue to state otherwise in popular discourse, such as the examples of Larry Summers and Tim Hunt, consensus across the research is that women are equally capable of math-intensive STEM work despite the fact that their underrepresentation specifically in these fields persists (Ceci \& Williams, 2010; NSF, 2015). The present study was also supported by the assumption that the students who 
participated in the study have experienced the factors that influence gender roles as they are constructed in STEM classrooms and professional spaces, whether or not they are conscious of these factors and these roles.

\section{Delimitations}

The study considered 900 undergraduate female students who were majoring in math-intensive STEM majors such as physics and physics-related fields (i.e., engineering, astrophysics, advanced mathematics) at an American university at the time when they completed the APS sponsored 2016 CUWiP pre-conference survey. This national dataset was facilitated by the American Physical Society (APS) and approved by the University of Maryland College Park IRB (Project \# 505475-10; see Appendix A). Furthermore, this study was limited to the 18 qualitative participants who were purposefully selected from the 9002016 CUWiP respondents. I chose not to include students in other STEM majors such as biology, chemistry, nursing and other health sciences because gender equity in those STEM areas is comprised of different dynamics (e.g., gender equity at the academic levels juxtaposed with gender inequities in professional leadership positions and tenured faculty positions in the same fields) (NSF, 2015).

\section{Definitions of Terms}

The terms defined below were used throughout this work:

- Agentic: as defined by Diekman et al. (2010) is having agency over one's own life, role, and actions; this term is used to describe traditionally male roles and characteristics such as power, achievement, independence, and selforientation. 
- Communal: as defined by Diekman et al. is oriented toward roles and characteristics which are traditionally other-oriented, such as helping others, serving the community, and working with people; this term is used to describe traditionally female roles and characteristics (2010).

- AAAS: American Association for the Advancement of Science, http://www.aaas.org/

- AAUW: American Association of University Women, http://www.aauw.org/

- APS: American Physical Society, http://www.aps.org/

- CUWiP: Conferences for University Women in Physics, sponsored by APS. These conferences take place on the same dates at nine locations across the U.S. to expose female undergraduate physics majors to professional development and networking within the field of physics. http://www.aps.org/programs/women/workshops/cuwip.cfm

- Gender: the socially constructed identities that further define a person's biologically assigned sex of male and female to manifest as masculine and feminine (Bem, 1981).

- Gender Roles: the socially constructed roles prescribed to people based on their sex and perceived gender (Bem, 1981; Diekman et al., 2010).

- Gender Role Congruity: specific dimensions of gender, such as agency and communal, commonly accepted at the group level that can create prejudices impacting women's participation in roles that embody the dimensions of what it means to be male, and vice versa (Eagly \& Diekman, 2005). 
- Higher Education: Any institution offering degrees beyond the equivalent of the high school diploma (e.g. associates degrees, bachelor's degrees, undergraduate certificates, graduate certificates, master's degrees, medical degrees, law degrees, doctoral degrees).

- “Ideal” Scientist/Science Student Concept: Adapted from Sader's (2011) work that theorized that for a woman to choose to pursue a male-dominated science field, she must use the same language to describe herself as she does to define a successful scientist, as well as from Shanahan and Nieswandt's (2010) work which found that students and teachers alike view "ideal" science students and scientists as suitable roles for those with certain characteristics, skill level, and sense of agency.

- Intersectional/Intersectionality: A term used to describe the phenomenon that examination of a social issue from the perspective of only one identity model (e.g. violence against women through an exclusively gendered lens) is inadequate (Cho \& Crenshaw, 2013; Crenshaw, 1991). The term itself is attributed to legal scholar Kimberlé Crenshaw, and has been widely accepted by researchers as an integral point to consider when conducting research related to oppression, violence, discrimination, and other social injustices (Collins, 2009; Jones \& Wijeyesinghe, 2011; McCall, 2005). While this lens was not the focus of this study, it has been touched upon occasionally.

- IRB: The Internal Review Board, a process by which research protocol and procedures are reviewed to ensure the safe and ethical treatment of study participants. 
- Math Intensive STEM Fields: the fields of science that involve high skill levels and competence in advanced mathematics including physics, engineering, and computer science (Ceci et al., 2009; Ceci \& Williams, 2010; NSF, 2015).

- NSF: National Science Foundation, http://www.nsf.gov/

- Persistence: The continuous pursuit in an academic program of study, or career path. For the purposes of this study, persistence will be measured by participants' self-reported intentions to persist in their academic endeavors and their willingness to self-report related future education and career plans (i.e., plans to persist).

- Physical Sciences: The sciences concerned with the study of inanimate natural objects, including physics, chemistry, astronomy, and Earth sciences.

- Physics Identity: The four dimensions commonly utilized to predict whether or not a student will self-identify as a physics person: feeling recognized, interest in science, performance, and competence (Carlone, 2004; Hazari et al., 2010).

- STEM: An acronym used as an umbrella term to describe both academic and professional pathways for science, engineering, technology, and mathematics.

- STEM Major: Indicates the specific major area of study a student can choose to pursue such as: Mathematics, Statistics, Computer Science, Information Technology, Information Systems, Computer Engineering, Civil Engineering, Biomedical Engineering, Mechanical Engineering, Environmental 
Engineering, Electrical Engineering, Physics, Biology, Chemistry, Earth Science, Geoscience, and Astronomy.

\section{Summary}

Chapter I provided a brief overview of the background of the problem, the problem statement, the purpose of this study, the research questions, the conceptual framework, the assumptions and delimitations, and the definition of terms used. In the next chapter, a more comprehensive review of the literature focused on women and minorities in STEM, women in physics, and the conceptual framework will be provided. 


\section{CHAPTER II}

\section{REVIEW OF THE LITERATURE}

\section{The STEM Dilemma}

Increasing attention has been paid to the education of students engaged in science, technology, engineering, and math (STEM) for decades. The body of literature expressing a collective desire to emphasize recruitment and retention of "others" into math intensive fields traditionally dominated by White and Asian males such as engineering, physics, and computer science has increasingly focused on specific points of engagement where recruitment and retention may be problematic. For instance, Brotman and Moore (2008) synthesized the literature on the "gender gap" in these particular areas of science and engineering into four themes: "equity and access, curriculum and pedagogy, the nature and culture of science, and identity" (p. 971). Variations on these four themes have been examined by researchers whose interests in closing this gap are as diverse as the causes. Ceci, Williams, and Barnett (2009), and later Ceci and Williams (2010, 2011) categorized the various academic areas that have focused on this specific research endeavor the most into the follow seven categories: "endocrinology, economics, sociology, education, genetics, cognitive neuroscience, and psychology" (2010, p. xiii).

However, for the purposes of this analysis, since understanding the role higher education plays in closing equity gaps in STEM is among the desired outcomes, the majority of the literature utilized derives from the fields of education (i.e., higher education, educational leadership, science education, curriculum and instruction, and educational policy), and areas of study related to sociology and psychology (e.g., women's and gender studies, feminist justice research, psychosocial and cognitive 
identity formation and development, cultural and environmental influences, etc.).

Reasons for focusing the review of the literature in this manner include the intended outcomes of examining these factors, influences, and theoretical frameworks while simultaneously seeking opportunities to provide programmatic best practices for higher education administrators, student affairs practitioners, and faculty seeking informed ways to support and retain women in STEM majors.

Chapter II will cover who is underrepresented in STEM, and asks the question: Why diversify? Second, women's STEM pathways will be covered including: primary and secondary education, higher education, career and work; and women and physics. The final section of this chapter will situate the conceptual framework within this context in more detail and will close with a summary of this chapter and an overview of the next chapter.

\section{Who is Underrepresented?}

In a 2002 report, the NSF (National Science Foundation) noted that STEM fields were expected to grow by three times before 2010. In their Science and Education Indicators 2014 report, the NSF dedicates the second chapter to the role higher education plays in meeting this demand for an educated, skilled STEM workforce. Over the last 15 years, the number of science and engineering bachelor's degrees awarded has risen steadily, but the proportion of bachelor's degrees in these majors to other bachelor's degrees has remained consistent at 32\% (NSF, 2014). Thus, undergraduate degrees in STEM have risen at the same rate as all undergraduate degrees. Additionally, degreeseeking students in these areas at the master's level have increased by over $50 \%$ in the same time span, and this is in large part a result of the increase in student enrollment in 
these areas since the economic downturn, with the largest increases taking place in engineering, psychology, political science and public administration. The NSF also reported that international students on temporary visas earned $56 \%$ of all engineering doctorates, $51 \%$ of all computer science doctorates, $44 \%$ of physics doctorates, and $60 \%$ of all economics doctorates in 2011. In their collaborative 2015 report, the NSF, the National Center for Science and Engineering Statistics, and the Directorate for Social, Behavioral, and Economic Sciences reported that those who are continually underrepresented in some science and engineering fields include the three racial and ethnic groups, "blacks, Hispanics, and American Indians or Alaska Natives” as well as persons with disabilities and women (p. 2). They go on to report that despite women earning well over $50 \%$ of all bachelor's degrees for nearly 20 years, and about half of all science and engineering bachelor's degrees since the late 1990's, there is persistent gender disparity in engineering, computer sciences, and physics fields of study and work (p. 5). The University of Oklahoma's Center for Institutional Data Exchange and Analysis (C-IDEA) reported retention rates for underrepresented college students, specifically racial minorities, to be approximately $24 \%$ at a six year graduation rate as compared to $40 \%$ of White students (2000).

Among the researchers who seek to understand the complex issues of underrepresented groups in STEM, and the ways in which they intersect with fields of study and practice that are themselves complex, consensus exists in a few areas. First, there is agreement that the data reported by the NSF (2007, 2014, 2015), AAUW (Dyer, 2004), and other similar reporting bodies accurately reflects the demographics of students who pursue a STEM major beyond the required classes offered precollege, as well as the 
demographics of underrepresented students who complete these degrees at the bachelor's, master's and doctoral levels. Second, there is consensus that women have reached parity among degree seeking students in some STEM fields of study such as biology, medicine and other health fields that require a science background, as well as many of the social sciences (except economics where women are still underrepresented at 30\%, and psychology where they are overrepresented at 70\%) (NSF, 2015). In 2015, the AAUW dedicated an entire volume of their publication, Outlook, to the current state of this persistent disparity stating: "Most people seem to agree that there's a gender diversity crisis in the [STEM] fields - and the situation is especially alarming in engineering and computing. What people don't agree on is how to fix the problem” (Moulton Belec, 2015, p. 19).

Despite the often quoted and relatively recent 2005 speech by Harvard President at the time, Larry Summers, in which he speculated that perhaps the underrepresentation of women in these fields (especially at elite schools) is due to innate abilities of women which do not afford them the opportunities to participate in these fields, there is general acceptance - although not universal - that women have the same aptitudes, innate abilities, and potential to succeed in math intensive fields of study (AAAS, 2005). As a consequence of the fact that the majority of those who research the STEM gender gap do not consider the cause to be rooted in ability differences, there is an unspoken consensus across the literature that the contributing factors of the underrepresentation of women and minorities in certain STEM fields are rather more closely related to the themes identified by Brotman and Moore (2008), such as "the nature and culture, and identity" (p. 971); in other words, a series of internal and external factors that steer underrepresented groups 
away from these fields across their lifetime (Leslie et al., 2015; Rosenthal, London, Levy, \& Lobel, 2011). As many who have offered theories and research to explain these internal and external factors, an equal number of researchers have offered suggestions for closing the gap. As an example, according to Diekman et al.'s (2010) study:

It is ironic that STEM fields hold the key to helping many people, but are commonly regarded as antithetical (or at best, irrelevant) to such communal goals. However, the first step toward change is increasing knowledge about this belief and its consequences. (p. 1056)

\section{Why Diversify?}

The fact that there is persistent underrepresentation of certain groups in math intensive fields of science such as computer science, physics, and engineering (Ceci et al., 2009; Ceci \& Williams, 2010; NSF, 2015) means that individuals from these groups do not have access to all of the opportunities available for them to reach their full potential to contribute to these fields. Among the commonalities throughout the various feminist theories applied to this line of inquiry, many of which are latent or implied in the majority of this research (Beddoes \& Borrego, 2011; Nicholson \& Pasque, 2011; Niskode-Dossett, Pasque, \& Nicholson, 2011), is the sentiment that women who do not participate in the workforce at the same rates as men miss out on economic and professional benefits and ultimately: "The result of this exclusion is that the true potential of women's contributions to society is unfulfilled" (Nicholson \& Pasque, 2011, p. 5; also in McPherson \& Fuselier-Thompson, 2013; Tong, 2009).

According to Hughes (2010), "the past two decades of efforts to increase the number of women in STEM majors at the university level have not been completely successful” (p. 431). In fact, the persistent underrepresentation of women in some STEM 
areas also indicates that higher education has been complicit in perpetuating this gender gap. Despite increased research and scholarship on the varied and complex causes of this gender gap in some STEM areas, higher education has not made an across-the-board commitment to closing the gap. Support programs, curriculum reform, and interventions are being implemented on a campus-by-campus basis, which will be discussed in depth later in Chapter II, but there are few best practices yet to be widely accepted. The National Science Foundation (NSF) is committed to highlighting ways in which these gaps present themselves in STEM fields in higher education due to the institutions' responsibility and active engagement in creating knowledge, maintaining knowledge, and transferring knowledge to students and other constituents (2014).

The final topic of relative consensus in this area of study argued by researchers is that without equal representation in these fields, opportunities for innovation, creativity, and other advances are omitted (Shapiro \& Sax, 2011; Sadler et al., 2012; Oh \& Lewis, 2011; Obama, 2009). When a workforce does not adequately reflect the greater population, and everyone does not have equal opportunities to reach their full potential within those fields, it may not be possible for those fields to reach their full potential either. Therefore, the reasons to incorporate more diversity into STEM fields go far beyond a desire or need for increased inclusion, but rather are considered a matter of need for continued innovation and positive socio-economic impact (Chang, Eagan, Lin, \& Hurtado, 2011; Nasir, 2002; Carlone \& Johnson, 2007; Rahm, 2007). Aside from the well documented, and persistent, disparity of women in specific STEM fields, there is a growing body of literature focused towards increased attention to diversity, access, and inclusion efforts in these fields. 


\section{Women and STEM}

Gender difference in participation in many of these fields has been documented for decades (NSF, 2015; Solomon, 1997). With the increase in demand for a skilled STEM workforce and an output that has not kept pace with demand, issues of disparity and inequality in STEM have increasingly been brought to the forefront. Historically, research conducted by Clancy (1962), Kilson, (1976), Peng and Jaffe (1979), and Martin and Irvine (1982), and the American Association of University Women (2004, 2015) has reported on the issue of gender disparity in STEM fields for degree earners and professionals; what Faulkner deemed the, "Why so few?" question (2001, p.79). Turning our attention to the contemporary discourse, Shapiro and Sax have called for changes in the direction of research related to higher education and women in STEM because they argued that continued research on the underrepresentation of women persisting in STEM programs by numbers alone will not influence policy and teaching practices enough to adequately increase participation by women in these fields (2011).

The literature indicates that researchers today increasingly make inquiries and provide insights that expand the depth and complexity of these issues in ways that build upon the early questions of disparity while appearing vastly different from their predecessors. For example, Clancy (1962) documented observations of environmental factors that he thought might hinder women's participation in science, such as social pressures and feeling like an "outsider," thus impacting reduced numbers. In 1982, Hall and Sandler's research contemporized Clancy's claims when they produced a report on women's experiences in STEM classrooms and professional fields describing the climate 
as "chilly." Researchers today thoroughly explore specific constructs within this realm to uncover and articulate these nuances.

An area explored at length in this line of inquiry is that of the internal and external factors that contribute to the development of self-efficacy for women who choose to pursue STEM. Self-efficacy is a social cognitive theory which provides insight into the self-beliefs persons have about their capabilities when it comes to knowledge, skill, application, and performance (Bandura, 1997; Zeldin \& Pajares, 2000). Much of the selfefficacy research focuses on the individual and how their concept of self-efficacy forms, or fails to form. Some researchers have spent a great deal of time seeking the most influential source of self-efficacy for women in STEM fields. For example, Zeldin and Pajares (2000) conducted a case study of women who have successful careers in maledominated STEM fields to determine commonalities across their self-efficacy and selfconcept within their chosen fields. In general, they found that "women's perceptions of their capabilities to succeed in mathematics and related areas are significantly lower than those of men in the same areas" (p. 218). The finding has recently been confirmed by Hazari, Sadler, and Sonnert (2013) who found that women reported, "significantly lower self-perceptions toward physics, and Hispanic females tended to be the most disempowered in their views of themselves with respect to science" (p.82).

The themes reported in the Zeldin and Pajares findings included the importance of what they called 'vicarious experiences' and 'verbal persuasions' (2000, p. 227), and that the participants were able to recall those influences more often and in greater detail than their own personal accomplishments in their education and work. For every participant, they recalled exposure to the concepts of their fields early in their childhood (vicarious 
experiences), and the profound influences of encouragement from others (verbal persuasions), especially males such as family members and teachers. The finding is also echoed in the study conducted by Hazari et al. (2007) who listed among their findings the statistically significant influence of a father's encouragement on a female's interest and willingness to pursue physics.

Because of the "chilly climate" felt by women and minorities in these fields of study and work (Allan \& Madden, 2003; Fassinger \& Asay, 2006; Hall \& Sandler, 1982; Salter, 2003; Sandler, Silverberg, \& Hall, 1996; Urry, 2003), self-efficacy has served as a helpful backdrop for a number of studies on gender and STEM. It has been theorized that women who pursue these fields of study and work do so despite this chilly climate, and therefore exhibit aspects of self-efficacy within their chosen field that other women cannot access. Thus, self-efficacy serves as an example of an effective framework for researchers when other theories do not adequately account for the nuanced causes of retention and attrition rates of women in some STEM fields.

Other researchers have found different dimensions as helpful frameworks for these issues, such as identity development (Barton \& Tan, 2010; Callahan, Pyke, Shadle, \& Landrum, 2014; Chang et al., 2011; Faulkner, 2007; Kane, 2012; Potvin, Hazari, \& Lock, 2013), environmental factors ranging from stereotype threat (Chang et al., 2011; Perna et al., 2009; Steele, James, \& Chait Barnett, 2002) to reformed curriculum (Carlone, 2004; Hazari, Tai, \& Sadler, 2007), and support programs aimed at retention of women in these fields (Bystydzienski \& Brown, 2012; Johnson, 2012; Soldner, Rowan Kenyon; Inkelas; Garvey \& Robbins, 2012; Szelenyi, Desnon, \& Inkelas, 2013). Although the increasingly nuanced studies alone have not achieved equality across these 
fields, they provide contextual and dialogic space for a deeper discourse about the gender inequity challenges faced by STEM fields of study.

\section{Women in Physics}

As mentioned earlier, merely examining the gap between the number of men and women engaged in STEM work, or even the root causes of that gap, cannot be enough (Shapiro \& Sax, 2011); nor can examination of the women who have successfully persisted into STEM fields alone. Instead, Shapiro and Sax recommended more focused research on sub-populations with particular success or challenges within these fields. Research focused on women's participation in physics, for example, has noted the singularly objective concepts and work of physicists, leading many to describe the field as an equally cultureless, genderless field, juxtaposed with the gendered roles and need to neglect their gender identity or expression often described by female physicists in examples of their lived experiences in the classrooms, labs, and work places (Carlone, 2004; Gonsalves, 2014; Götschel, 2014).

Interestingly, within the field of physics, built upon objectivity so crystalized that inequality is considered impossible, many participants do not directly articulate the masculine nature of the field until prompted for specific examples (Gonsalves, 2014). In her study of female physics doctoral students, Gonsalves noted that nearly all of her female study participants defined themselves in opposition of femininity (2014).

Gonsalves also observed that in order to feel competent and recognized within their field, in addition to their credentials and experiences as physicists, the female participants upheld underlying cultural norms within the field such as sexist, heteronormative, and essentialist language. Many of the gendered roles and norms built into the culture of 
physics, which Gonsalves (2014) and Danielsson (2009) have agreed does in fact exist, are not considered consciously by those who elect this pathway. These specific concerns within this specific context have led this study to integrate the four-part conceptual framework chosen for this study.

\section{Situating the Conceptual Framework}

Many studies highlight the need to increase diverse representation among STEM students and the workforce in order to safeguard against homogeneity of ideas and innovations, which could potentially thwart progress in these areas of research and practice. Additionally, many studies focused on the "pipelines" and "pathways" for women in these fields indicate loss of interest and STEM identity development often times before they even have the opportunity to choose a STEM major in college (Brickhouse et al., 2000; Carlone, 2004; Espinosa, 2011; Faulkner, 2001, 2007). A consequence of the highly interdisciplinary, intersectional (Cho, Crenshaw, \& McCall, 2013; Crenshaw, 1991; Gee, 2000; Jones \& Wijeyesinghe, 2011; Mcall, 2005), and epistemological facets of this body of literature, an equally diverse set of theoretical frameworks are utilized to adequately convey the nuanced issues and the complexities of the findings. In order to convey thoughts and ideas at an abstract level with clarity, Anfara and Mertz (2006) described the diversity of frameworks available to researchers as a structured range which on one end has the concrete sensations and experiences, and on the other end the abstract propositions, ideological relationships, and theories. The majority of contemporary research on gender disparity in specific STEM fields relies on multiple frameworks in combination either within studies, or across a researcher's body of work. Although concrete explanations for the use of multiple concepts and theories 
within a study are not present in the most comprehensive reviews of the literature (Brotman \& Moore, 2008; Faulkner 2001, 2007), it can be theorized that the multiplicity in approaches researchers have utilized in this area is yet another indicator of the complex nature of the causes of the STEM gender gap.

And yet, there are some commonalities among the theoretical concepts underpinning the axiological issues of science education when gender equity is the focus. For example, some researchers utilize theoretical frameworks chosen to address the ideological and cultural contributors to gender disparity in STEM (Brotman \& Moore, 2008; Bystydzienski \& Brown, 2012; Carlone, 2004; Griffith, 2010; Solomon, 1997) while others have been focused on conceptualizing the overt and covert manifestations of bias and discrimination (Brickhouse et al., 2000; Ceci \& Williams, 2011; Diekman et al., 2010; Johnson, 2012; Oh \& Lewis, 2011) which discourage girls and women from participating, or cause them to believe that they are opting out of STEM fields of study. Because of these nuanced goals, coupled with the diverse contributors to the underrepresentation of women in STEM, it is not surprising that there are nearly as many combinations of frameworks available as there are studies.

Despite these complexities embodied in the disparity of women in STEM research, another commonality across the literature is the categorical tendencies of STEM research which in general represents a persistent desire for the concrete. A bifurcation of the complex issue to adequately categorize the concepts as a common theme can be observed in the line of inquiry. For example, the juxtaposition of men and women, and the majority and "minority" engaging in STEM are among the simplest dichotomies that create the foundation of what is being examined. Other examples of how researchers 
have accomplished bifurcation through theoretical frameworks include the common use of agentic and communal roles (Diekman \& Eagly, 2000; Eagly \& Karau, 2002), the examination of role congruity or incongruity for women in STEM (Diekman \& Goodfriend, 2006), and the internal and external factors influencing women's participation in these fields (Rosenthal et al., 2011; Zeldin \& Pajares, 2000). There is even a sense of tension between the dichotomy of social justice and tradition across these fields, and the invisible pull of subversion and obedience across the literature. A fascinating aspect of these bifurcated abstractions used to examine gender disparity in STEM is that the arguments silently mirrors the existential feminism prescribed by Simone de Beauvoir (1949/2009) and many other feminist theorists and researchers who have spent their careers defining the dichotomy of the public and private spheres in which gender roles are constructed. Interestingly, many studies examining underrepresented groups in STEM use science, postpositivism, and pragmatism to concretize the dialectic nature of disparities caused by intersecting components. Perhaps researchers are using these concrete, postpositivist approaches any scientist in these STEM fields would use, know, and understand to describe the pervasive dichotomies that frame these issues.

Beyond these observations about the use of theoretical and conceptual frameworks in this line of inquiry, there are a few concepts utilized regularly. For example, identity formation and development is the focus of many researchers (Brickhouse et al., 2000; Callahan, Shadle \& Landrum, 2014; Carlone \& Johnson, 2007; Chang et al., 2011; Cob, 2004; Faulkner, 2007; Hazari et al., 2010; Potvin, Hazari, \& Lock, 2014; Rahm, 2007; Rosenthal et al., 2011). Other examples of common frameworks include: gender role congruity (Archer et al., 2012; Archer et al., 2013; 
Diekman et al., 2010; Johnston \& Diekman, 2015; Gonsalves, 2014; Gotschel, 2014), self-efficacy (Bandura, 1997; Zeldin \& Pajares, 2000), environmental factors and motivation (Carlone, 2004; Espinosa, 2011; Griffith, 2010; Hazari, Cass, \& Beattie, 2015; Sansone \& Harackiewicz, 2000; Salter, 2003), sense of belongingness (Goodenow, 1993; Kawachi \& Berkman, 2001; Lee \& Robbins, 1995), and the effectiveness of support programs and interventions (Hughs, 2010; Potvin, Hazari, \& Lock, 2014; Salto, Riggs, Delgado De Leon, Casiano, \& De Leon, 2014; Rosenthal et al., 2011; Szelenyi et al., 2013). As mentioned in Chapter I, a more detailed account of the composition of the conceptual framework including the use of a physics identity theory as defined by Hazari et al. (2013), role congruity theory as defined by Eagly and Diekman (2005), a concept of the "ideal" scientist (Sader, 2011; Shanahan \& Nieswandt, 2010), and the hypothesized, combined impact of these on persistence will be expounded upon below.

\section{Physics Identity Theory}

Extensive research has been done to better understand identity development for female college students, as well as the impact this can have on whether women choose a STEM major in college or not. In 2007, Faulkner published a piece about the extensive identity "co-construction" work women in male-dominated STEM fields must do in order to persist in the field and be seen by others as competent experts. She wrote: "[I]t seems the gender authenticity issue never quite goes away for women in a male-dominated occupation - even though women engineers clearly are building new co-constructions of gender and engineering identities" (p. 349). The reasons for bringing attention to this issue specifically she explained as: 
Significantly, whereas these women are, in effect, creating new gender identities as women engineers, their male colleagues do not have to do equivalent gender work....For women engineers, tensions can flow from the very "gender inauthenticity" of the woman engineer, which means that women engineers have a constant struggle to prove that they are not only "real engineers" but also "real women." (p. 350)

To represent the intersectional nature of identity development, some of the well-known gender identity development theoretical models often cited in this work include Carol Gilligan's theory on women's moral development (1982/1993), and the theory of women's ways of knowing offered by Belenky, Clinchy, Goldberger, and Tarule (1986). The theories can be applied to illuminate contextual perspectives for women in maledominated STEM fields such as physics. If women's identity development of morality and knowledge form in connection to both external and internal influences as these theories posit, then this is aligned with arguments for identity as the focus in STEM and gender work also. In lieu of using either of these gender theories as a cornerstone of this study, I have instead chosen to take the conceptual model of this study in a direction that echoes the sentiments of Faulkner (2007): Namely, to highlight the physics identity model proposed by Hazari et al. (2010) because it offers natural points of intersection with the underrepresentation of women in this particular STEM field, as well as the concept of gender role congruity, and the concept of the "ideal" scientist.

Utilizing a science identity lens is an approach familiar to researchers addressing various aspects of STEM education such as curriculum and instruction, underrepresented students, and engagement (Carlone, 2003, 2004, 2007; Cobb, 2004; Nasir, 2002; Tonso, 2006; Rahm, 2007). Since science identity captures a more comprehensive view of how a person becomes a scientist, an engineer, or a mathematician, beyond the teaching and 
learning of knowledge, skills, and abilities in these fields, but also including their experiences and perceptions of themselves and who they can become in their chosen field, it is a useful theoretical framework for capturing the cultural aspects of STEM fields that can serve as barriers for underrepresented students (Brickhouse, Lowery, \& Schultz, 2000; Carlone \& Johnson, 2007; Hazari et al., 2010). Additionally, unlike theoretical frameworks focused on the curriculum development, or the learning process, an identity lens, "allows us to ask questions about the kinds of people promoted and marginalized by science teaching and learning practices" (Carlone \& Johnson, 2007, p. 1189).

Identity formation and development encompasses many qualities within the context of gender and STEM in higher education including cognitive and psychosocial; some themes transverse the literature on points of identity intersection such as gender, race, ability, college student identity, and STEM (Gee, 2000; Hazari et al., 2013). On the one hand, researchers use identity development to understand the ways students develop (or fail to develop) a STEM identity (Brotman \& Moore, 2008; Callahan, Pyke, Shadle, \& Landrum, 2014; Faulkner, 2001 \&2007; Potvin, Hazari, \& Lock, 2014). Others have built upon this work in detail to determine what exactly influences the creation of a STEM identity, and whether or not there are interventions available that can positively influence the STEM identity development of women in ways that encourage them to pursue the field. For example, Hazari, Sonnert, Sadler, and Shanahan (2010) utilized a national data set from the Persistence Research in Science and Engineering (PRiSE) project $(n=3,829)$, which surveyed students enrolled in a bachelor's level introductory English course at 34 US colleges and universities about their high school science 
experiences and attitudes towards science to determine how a student's physics identity might be shaped by their high school physics education experiences and their future career goals. Multiple regression was used to find that the presence of a physics identity could be used to predict a students' intentions to pursue a physics career, and furthermore to determine if there are any additional factors that affect the development of a physics identity for female students. Another interesting detail of their findings is that they found a positive impact on the physics identity of female students when they were exposed to open discussion of the under-representation of women in science. These same discussion topics had no impact for the male students (Hazari et al., 2010).

In another study utilizing a larger PRiSE data set $(n=7,505)$, Hazari, Potvin, Lock, Lung, Sonnert, and Sadler (2013) went on to test the findings discussed above to determine which of the following factors impact female students' interest in a career in the physical sciences: having a single-sex physics class, having a female physics teacher, having female scientist guest speakers in physics class, discussing the work of female scientists in physics class, and discussing the underrepresentation of women in physics class. Again, Hazari et al. found that talking about underrepresentation was the only factor with a significant positive effect on the female respondents' intentions to consider a physics major or career. Therefore, there may be benefits to exposing students to this information on a large scale because it may have a positive impact on the physics identity development of female students. Even if large-scale implementation of this practice does not yield a significant effect for the male students, these studies also confirm that this practice does not have a negative effect on male students' ability to form their own physics identity (Hazari et al., 2013). 
According to Hazari et al. (2010) specific constructs can lead either to the successful, or unsuccessful, development of a physics identity. The researchers frame physics identity as the self-perception of a student as a physics "person" (Hazari et al., 2013), because they do not use this term to mean a, "physics practitioner identity, or a physicist identity but rather how students designate themselves with respect to physics in the context of their experiences (mostly as physics students)" (Hazari, Cass, \& Beattie, 2015). Utilizing the identity constructs of performance, competence, and recognition reported by Carlone and Johnson in 2007 as the basis, and then contributing an additional construct of interest (Hazari et al., 2010), they have found that a physics identity can best be predicted by the following three constructs: feeling recognized, their interest in science, and feeling competent (Hazari et al., 2013). Figure 3 provides a visual depiction of these dimensions for additional understanding of this specific look at identity:

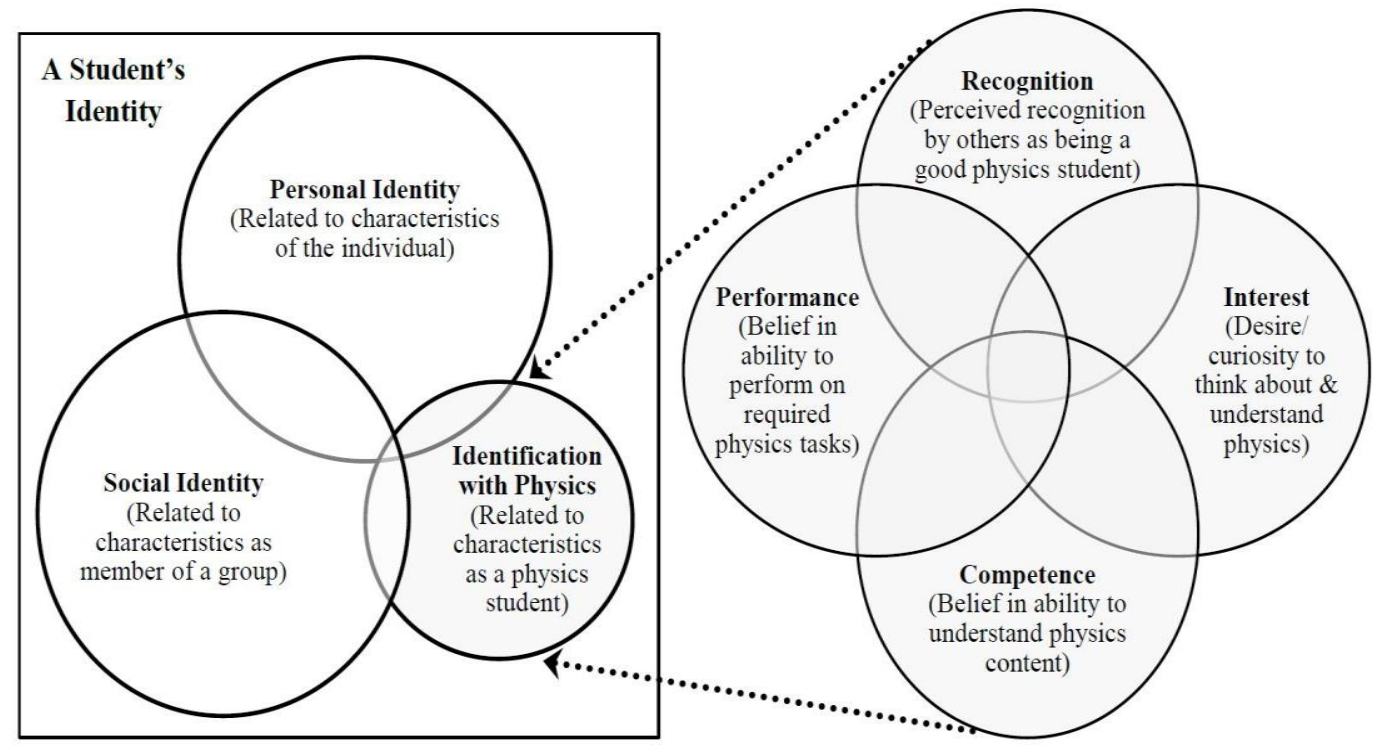

Figure 3. Framework for students' identification with physics. 
Placed in context, Hazari et al. (2010) found that the constructs of a physics identity have different implications for female and male students. For example, their data analysis showed that a physics identity, "correlated positively with a desire for an intrinsically fulfilling career and negatively with a desire for personal/family time and opportunities to work with others" (2010, p. 978). If female students are seeking a career field and academic major that allows them to have an intrinsically fulfilling career and time for opportunities to work with others and fulfill personal and familial goals, then an inherent barrier exists for female students when it comes to the development of a physics identity. However, because female students do pursue physics as a major and as a career path despite this and other barriers, additional frameworks in combination with the student's physics identity aids in conveying critical nuances that make up many of the other barriers for women entering the field of physics in particular.

\section{Gender Role Congruity Theory}

Role congruity theory (Eagly \& Diekman, 2005) can be applied to the prejudice against women in any context that is traditionally male-dominated including executive leadership (Eagly \& Karau, 2002), and STEM (Diekman, Brown, Johnston, \& Clark, 2010; Diekman \& Goodfriend, 2006). Similar to the identity conflicts examined by

Faulkner (2000, 2007), role congruity examines the:

[P]erceived incongruity between the female gender role and leadership roles [that] leads to 2 forms of prejudice: (a) perceiving women less favorably than men as potential occupants of leadership roles and (b) evaluating behavior that fulfills the prescriptions of a leader role less favorably when it is enacted by a woman. (Eagly \& Karau, 2002, p. 573)

Figure 4 provides a list of these dimensions of gender defined: 


\begin{tabular}{|l|l|}
\hline Agentic goals $(\alpha=.87)$ & Communal goals $(\alpha=.84)$ \\
\hline Power & Helping others \\
Recognition & Serving humanity \\
Achievement & Serving community \\
Mastery & Working with people \\
Self-promotion & Connection with others \\
Independence & Attending to others \\
Individualism & Caring for others \\
Status & Intimacy \\
Focus on the self & Spiritual rewards \\
Success & \\
Financial rewards & \\
Self-direction & \\
Demonstrating skills or competence & \\
Competition & \\
\hline
\end{tabular}

Figure 4. Resulting goal-endorsement factors for agentic and communal goals. A factor analysis of goal-endorsement items supported two distinct factors: agentic goals and communal goals. Cronbach's alphas indicate high internal consistency within each scale. The items in the agentic goals column represent characteristics of traditionally maleoriented goals commonly accepted at the group level. The items in the communal goals column represent characteristics of traditionally female-oriented goals commonly accepted at the group level. Adapted from "Seeking Congruity Between Goals and Roles: A New Look at Why Women Opt Out of Science, Technology, Engineering, and Mathematics Careers," by A. B. Diekman, E. R. Brown, A. M. Johnston and E. K. Clark, 2010, Psychological Science, 21, p. 1054. Copyright 2010 by Sage Publications.

The examination of gender roles as constructed within this theoretical context provides insight into the "normative" expectations of the roles that manifest from the constructs. Thus, Eagly and Karau (2002) reference Cialdini and Trost's (1998) terms to further define these concepts: "descriptive norms, which are consensual expectations about what members of a group actually do, and injunctive norms, which are consensual expectations about what a group of people ought to do or ideally would do" (p. 574). These dimensions provide theoretical context for the expectations that, "a group will be positively evaluated when its characteristics are perceived to align with the requirements of the group's typical social roles" (Diekman \& Goodfriend, 2006, p. 369). Applying this 
to math intensive, male-dominated science fields such as physics, it is clear that the male roles and measures of success fall into what Eagly and Karau (2002) categorized as "agentic" (p. 574); successful participants in these fields are primarily ambitious, independent, self-confident, assertive, controlling, and dominant. However, women tend to either self-select into, or be encouraged into roles that more social, and what the researchers categorized as "communal" (p. 574) which includes being concerned with the welfare of others, a desire to help others, and a tendency toward being affectionate, sympathetic, and nurturing.

Although we know that not all men can be described as being agentic, and that not all women feel comfortable in roles categorized as communal, Eagly and Karau argue that these bifurcated roles are innately gendered, thereby causing gendered implications when applied in educational, professional, or research contexts in many fields. The authors reference Hall and Carter (1999) who, "showed that as behaviors become more sex differentiated in actuality (as assessed by meta-analytic data), people judge them as increasingly appropriate for only one sex" (p. 574). It is particularly helpful for this study because if both men and women consider physics to fulfill agentic roles, and lack examples that support communal roles in these fields, women who choose to pursue these fields must either co-create their gender and science identities as Faulkner argued (2001, 2007), approach their work with pre-determined congruities existing between their identities as Sader observed (2011), or reject their gender identity altogether as some of the participants in Jorgenson's study expressed (2002). Furthermore, women may find participating in these decidedly agentic spaces such as required physics classes in high school, or required calculus courses for pre-med majors, very uncomfortable for reasons 
beyond their perceptions of a "chilly climate" (Bystydzienski \& Brown; 2012; Carlone, 2004; Diekman, Brown, Johnston, \& Clark, 2010; Johnson, 2007; Sax, 1994). Women may be experiencing their own internal "chilly climate" due to their own awareness of role incongruity in spaces they perceive to be meant for someone else such as their male peers (Diekman et al., 2010). For these reasons, Diekman et al. wrote:

We propose that women's communal goal orientation intersects with beliefs that STEM careers do not involve helping or working with other people, with the results that even scientifically talented women frequently choose other careers - ones they believe will allow them to fulfill their communal goals. (2010, p. 1052)

Therefore, further exploration of the connections between these theories warrants attention. For example, there is an intersecting point between the role congruity theory (Diekman et al., 2010), and the physics identity theory (Hazari et al., 2010, 2013), and that is recognition. Although recognition is listed as a goal-endorsement factor for agentic goals, there is a consistency between this point and the construct of feeling recognized which impacts a student's physics identity development. The consistency across these theories leads to the research questions posed in this study: Is the way undergraduate women in physics see the "ideal" physics student related to the way they see their physics identity? And, how does gender role congruity relate to physics identity? Furthermore, following this train of thought a priori, another research question is posed to tie all of the concepts together: What factors (i.e., science identity, gender role congruity) relate to plans to persist?

\section{"Ideal" Scientist Concept}

Agreement that factors impacting the underrepresentation of women in some STEM fields are internal to the individual (e.g., their science identity), external to the 
individual (e.g., messages that define who is meant for science, and who is not), and at times both (e.g., gender role congruity is defined externally and manifests internally in different ways for different individuals) exists in this literature (Cobb, 2004; Diekman et al., 2010; Hazari et al., 2010; Leslie et al., 2015). Therefore, the third concept which will be used in this study captures the external messages students receive through the educational and socialization process regarding which academic disciplines are appropriate for whom through a lens different from gender role congruity theory. The "ideal" scientist (and therefore the "ideal" science student who is capable of becoming the "ideal" scientist) is known in different ways by different researchers. For Leslie et al., they have termed this, "the field-specific ability beliefs hypothesis" (2015, p. 262), which represents a shared understanding that some academic disciplines require inherent aptitudes. In addition to Leslie et al.'s response to this phenomenon, Shanahan and Nieswandt (2010) and Sader (2011) have also explored this concept.

Shanahan and Nieswandt (2010) recommend the consideration of this paradigm when research specific to science education employs student agency and identity variables, as this study does. In their mixed methods study of 95 qualitative participants and 157 quantitative participants (in that order), students were asked what factors make up the definition of the role of a science student. Participants were able to articulate the role of the science student well, regardless of whether they were enrolled at the same school as other participants, or had taken the same science curriculum as other participants. Because of this finding that neither the instructor, the curriculum, nor the geographic location changed how students described the role of the science student, Shanahan and Nieswandt's data provide evidence that science classrooms are governed, 
however implicitly, by social structures that create framework for, and stereotypes about, the role a successful science student plays in that environment. Additionally, they found that students can articulate role understandings among students in science classes, “characterized by references to expectations of intelligence, experimental skill, scientific mindedness, and appropriate classroom behavior" (Shanahan \& Nieswandt, 2010, p. 367). The findings have been confirmed by Bian et al. (2017) in that students become conscious of related implicit social expectations and assumptions as early as 6 years old. In the same vein, Jennifer Sader shared her findings from interviews with 10 doctoral students pursuing computer science degrees at four different higher education institutions in the chapter she authored in Empowering Women in Higher Education and Student Affairs (Pasque \& Nicholson, 2011), (2011). Sader utilized a theory of gender construction to express how "gender schemas" (Bem, 1981; Valian, 1999) impact the way people see themselves and others in relation to their gender: "These ideas shape our lives, including what we imagine as possibilities for ourselves, what our relationships are like, and what others expect of us" (Sader, 2011, p. 122). Among her findings, Sader noted that for women in the male-dominated field of computer science, specifically at the graduate level, their gender identity and their STEM identity seemingly had to be congruent in order for the women she interviewed to persist toward their goals. Sader went beyond the concept of identity co-construction to posit that there could be implications if a relationship exists between the language a woman uses to describe a successful scientist and the language she uses to describe herself, thus the link between identity and persistence. 
For all of her participants, Sader observed that their STEM identity (particularly as a computer scientist in this case) and their gender identity "had to fit together," otherwise the participant would not have chosen to pursue the field (Sader, 2011, p. 125). These findings align with two of the three types of science identity as defined by Carlone and Johnson (2007), the "research scientist" and the "altruistic scientist," which are commonly referenced in this field. However, it is important to note that Sader's findings are not representative of all women in computer science, or even STEM, as evidenced by the contradictory experiences of those with the third type of science identity, the “disrupted scientist” (Carlone \& Johnson, 2007). The disrupted scientist identity is experienced by those women who do persist in their chosen STEM field - although not always - but when they describe who they are as scientists or science students, they often focus on, "experiences where they felt overlooked, neglected, or discriminated against by meaningful others" (p. 1202). The disrupted scientist identity was not among the voices shared through Sader's findings, but is noteworthy nonetheless.

The present study will build upon the intersecting point of congruence between the way a student defines themselves and the way they define successful role models in their field as the "ideal" scientist concept will be utilized. The concept will also be used interchangeably with the "ideal" science student, because logically students perceive "ideal" science students to aspire to, and be well suited for the role of "ideal" scientist in the future. According to Leslie et al., female students are impacted by messages about the inherent aptitudes required for certain academic disciplines differently than male students, resulting in their underrepresentation in fields perceived to have inherent aptitude requirements. 


\section{Women's Persistence on the STEM Pathway}

When does the typical female leave the STEM pipeline, or what is commonly accepted now as the "pathway" (Espinosa, 2011)? Is it during childhood when the toys of girls and boys begin to construct their skills and gender role expectations? Or, is it in middle and high school when girls formally enter new developmental stages of gender role expectations and identity formation, become aware of beliefs (mythologies) of which fields support work-family balance, and are exposed to their first conscious experiences with the "chilly climate" perpetuated by instructors, classmates, or parents (Farenga \& Joyce, 1999; Jones \& Wijeyesinghe, 2011; Joyce \& Farenga, 1999)? The developmental process into a college major or career was traditionally called a pipeline, but in this context of persistence and an enduring gender gap in STEM fields, it has recently been framed instead as a pathway. Some of the reasons for this include references in the literature to how little the route women take to become STEM professionals resembles a pipeline, such as when Epstein described the science pipeline in the U.S. as, "leakier than warped rubber tubing" (2006, p. 1). A pipeline is something followed in response to the forces of gravity and pressure of what is flowing within, and many researchers agree that this hardly describes the experience of women and other underrepresented groups in STEM. Comparatively, a pathway is a route laden with choices, options, and those who have navigated the options before you; the symbol of the pathway calls upon a decidedly more agentic image.

Although women leave STEM pathways as they change their majors in college, as they decide whether or not to attend graduate school, and as they make decisions about their career fields, there is consensus across the literature that the largest number of 
women leave the STEM pathway, or choose fields that require less math intensive preparation, as they enter college (Clark Blickenstaff, 2005; Chen, 2009; Griffith, 2010; Sadler et al., 2012; Shapiro \& Sax, 2011). Many students remove themselves from the STEM pathway as they enter college before they have had the chance to learn about all of the career options or whether they would actually be successful in those fields (Hazari et al., 2010; Sadler et al., 2012). Because researchers have identified significant exit points for women in STEM at all of these stages, exit points on the pathway aside from the high school to college transition can be challenging to pinpoint making decisions about how to focus resources to facilitate intervention efforts aimed at retention for women in these fields equally challenging (Clark Blickenstaff, 2005).

Primary and secondary education. An example of the complexity of these issues faced by students prior to higher education can be seen in Carlone's (2004) discussion of the female students who participated in her study who resisted an Active Physics curriculum in an upper-middle class high school because of the level of risk the students must take to be successful in this new curriculum, and the ways in which these risks directly challenge the "good girl as good student" identity that many of the female students brought to the class. This "good girl" identity as equivalent to the "good student" expresses how challenging it may be for female students to take more risks because risks require failure, and failure may not be comfortable for "good students." Particularly if the academic discipline is stereotyped as not for the student, this additional risk may create anxiety internally which may manifest as resistance externally. For these reasons, it is assumed that many of the female students who do go on to choose a STEM 
major in college have already challenged themselves beyond these gendered barriers (Archer et al., 2012, 2013; Baker, 2013; Carlone, 2004).

In a 2012 mixed-methods study, Bystydzienski and Brown articulated the specific ways that women and girls "perform gender" in a high school engineering program called, the Female Recruits Explore Engineering (FREE) project, which identifies "tenthgrade girls with strong academic records in mathematics and science at ten high schools" in the mid-west as predictors of their success in engineering (p. 6). The researchers found that engineering as a major, and as a career choice, is entrenched in hegemonic masculinity before engineers enter the workforce. Furthermore, these spaces are designed by, for, and around masculine values of success and achievement as early as high school engineering projects for school and competition.

Bystydzienski and Brown (2012) also found that the way that academic programs attempt to recruit more females into their ranks is perceived as unrealistic or forced to the girls in the FREE program, thereby creating yet another barrier. It may not be possible to increase the number of women in engineering fields unless major changes to the roles they play in these areas are seen as viable.

Social construction of gendered identities and portrayals of STEM work contribute significantly to the ways female students choose to major in these areas in college. Shapiro and Sax (2012) argued that research and literature points to the way that these choices are often made long before college. When these choices are made throughout the middle and high school education process, women who do choose a STEM major often find themselves ill equipped for the competitive structure of the students' evaluations within the spaces of the classroom and through coursework. 
Some of the challenges women face within these majors connect with broader expectations placed on women and girls from a cultural perspective. As Carlone (2004) saw evidence of the "good girl as good student" perspective from the girls in the high school Active Physics class she studied, many women are faced with doubts about their choice of STEM major when they receive grades that are considered "normal" for STEM majors (i.e., it is common practice for STEM professors to grade on a curve in which a letter grade of "C" is average, and therefore assigned to the majority of the class regardless of how they score on the exams; Griffith, 2010), but considered "abnormal" for good girls who are also good students. As Griffith (2010) noted, women who experience these tough grading scales may perceive this as a threat to their competency which may engage symptoms similar to The Imposter Syndrome (Clance \& Imes, 1978), and may cause them to retreat to areas of study and work that reduce their exposure to criticism that is perceived as critical of them as individuals (Jones, 2010).

Another gender-specific challenge female students may face within STEM include what both Faulkner (2007) and Bystydzienski and Brown (2012) articulated as innately masculine spaces as evidenced by how women participate in them; namely, women who find themselves in "boys clubs" when working on engineering projects often take on, or are assigned, traditionally feminine roles such as team management and organization (i.e., team secretary, note taker, report writer), and "rarely complete mechanical tasks" (Bystdzienski \& Brown, 2012, p. 15-16). Results of playing these roles in STEM experiments and team work over time can result in reduced skill levels, reduced self-efficacy within these spaces, underdeveloped STEM identities, and reduced interest, to name a few examples (Carlone, 2004; Bystdzienski \& Brown, 2012; Jorgenson, 2002; 
Perna et al., 2009; Zeldin \& Pajares, 2000). With these strikes against them, it may not be mysterious at all that many women do not choose math intensive STEM fields such as engineering, computer science, or physics, where they may expect these issues to persist regardless of whether or not they are conscious of the effects of role incongruity discussed by Diekman et al. (2010).

Higher education. Focusing attention on the strategies higher education administrators and faculty choose to utilize when addressing this phenomenon provides insight into how many colleges and universities have focused less on dictating the culture of the classroom and more on resources and programming that support the persistence of their female students in STEM majors (Brower \& Inkelas, 2007; Griffith, 2010; Perna et al., 2009; Soldner, Rowan-Kenyon, Inkelas, Garvey, \& Robbins, 2012; Szelenyi et al., 2013). According to Griffith (2010), some of the institutional factors that contribute to this choice include academic freedom, the tenure and promotion process for faculty, an emphasis on resource allocation to undergraduate-focused STEM education, and whether or not a significant focus on undergraduate education is present as measured by the ratio of undergraduate students to graduate students, as well as the amount of time and effort faculty devote to developing and reforming undergraduate education. Some researchers have chosen to look at the persistence of female STEM majors within single-sex programs to determine if there are factors that contribute to success in that context that can be applied elsewhere. For example, Perna et al. (2009) utilized the setting of Spelman College, an all-women HBCU to analyze gendered constructs within STEM majors in order to offer alternative perspectives on these issues as compared with studies that utilize samples from co-ed and predominately white institutions. 
The four major themes identified by Perna et al. (2009) through analysis of the data collected from focus groups of students and faculty included students' intentional choice of Spelman because of its reputation in supporting women in STEM fields, students' high level of interest in pursuing a STEM field at Spelman, the "academic, psychological, and financial barriers" experienced by the students, and finally the negative impact of these barriers for Black and African American women in STEM fields (p. 8). Among the most interesting findings of the case study were the intense levels of support faculty provided women in STEM majors at Spelman which ranged from small class sizes, to personalized directed research opportunities for students, to individualized advising recommendations which may be unique to students at Spelman due to its size and high-achieving student body. Using the all-female model of Spelman as a guide, research and practice at other institution-types may benefit from the issues raised through this example.

In another example of a single sex program at a mid-sized university in the northeast of the U.S., the Women in Science and Engineering (WISE) was the source of a sample of students studied to determine which internal and external factors affecting their self-efficacy in their chosen STEM major (Rosenthal et al., 2011). The two internal factors identified were the gender and STEM identities developed by the students, and whether these identity development processes were congruent, or in conflict. The external factors measured were the level of social support the students received through WISE. The authors note their particular interest in the identity development for the students both as women and as STEM students, because they posited that these two identities are often incongruent in U.S. culture, and therefore may either develop in 
incomplete ways, in competition with each other, or may be performed in compartmentalized ways by the students, thereby not forming concrete points of intersection between the identities. The level of perceived external support available may facilitate or hinder the creation of these points of intersection between their gender and STEM identities for female students working in the male-dominated spaces of the STEM classrooms and research facilities.

According to their data analysis, Rosenthal et al. (2011) stated, "single-sex programs might successfully focus on identity compatibility and social support to increase engagement of college women in STEM majors" (p. 733). The sociological approach to this study captured the identity development pieces often central to the program development and implementation goals sought by university administration. By articulating the points of identity conflict and dissonance, and the lack of intersectionality for students with a gender and a specific STEM identity, Rosenthal et al. have clearly addressed some of the internal root barriers for women in these majors beyond lack of self-esteem or self-efficacy: "Perceiving an incompatibility between one's gender and STEM identity can be a significant impediment to sustained achievement and engagement in pursuing a STEM career for women over time" (2011, p. 727).

In another example of the factors influencing the ways in which women persist to graduation in STEM majors, Cole and Espinoza (2008) explore the influential factors through conceptual frameworks focused on cultural competence and cultural capital gained prior to the students' college experience. Through this lens, they sought to determine what internal and external factors could be utilized to predict Latina success in STEM majors. They identified factors that positively influence the success of Latina 
students in STEM fields, and in the case of factors such as studying with other students and attending campus diversity events, they found factors that negatively influence the success of these students in STEM majors. Some of the factors Cole and Espinoza (2008) found to be most influential in positively predicting the success of these students were faculty support, a feeling of affinity and acceptance on campus, and a high GPA in STEM coursework in High School. When it came to their level of "parental education as a pretest variable, there was no significant impact on GPA" in college for Latina students (p. 296). Of those variables with statistically significant impact on the persistence and success of the students, the authors point out that while preparation and success in STEM coursework is often high for Latina students - meaning that those who choose a STEM major are well-prepared, highly interested in pursuing their chosen degree, and are often successful in completing the coursework at a high standard - it is important to note the correlation between their ability to persist in their STEM major and external factors connected with their own levels of cultural capital they bring with them to college environments.

One thing that is considered unique to the experiences of Latinas is the often strong sense of familial ties, and the ways that these ties shape their support systems through academically rigorous experiences such as STEM courses. According to Cole and Espinoza (2008), this nuance is somewhat unique to this subculture, because while they may have the support systems necessary, the skills and abilities, and even the selfefficacy to persist to graduation in a STEM major, external factors such as racism and sexism serve as consistent barriers. This finding is echoed in the work of Hazari et al. (2013) who found that of all underrepresented minorities, "Hispanic females may face the 
greatest challenges in developing a science identity" (p. 89). It is important to consider these unique needs, especially for practitioners and faculty at Hispanic Serving Institutions who may find these nuances either magnified for some subcultures within the Hispanic subculture, or conversely, may find some aspects of these issues invisible when Hispanic females are already a part of a dominant culture on campus (Cole \& Espinoza, 2008).

One final category of factors that may contribute to why female STEM majors in higher education persist in their major or not is noted by Griffith (2010). A large number of female faculty members in a STEM department might appear to role model gender equality for students thereby positively impacting female students' persistence; however, on average female faculty are more likely to hold positions at lower-levels than their male counterparts. According to Etzkowitz, Kemelgo, and Uzzi (2000), both male and female advisors can be unsupportive of female students; therefore the number of female faculty alone is not a solution to the problem of underrepresentation in STEM fields. A high number of inexperienced female faculty members and instructors and adjunct positions that are not tenure track may be the consequence of cultural barriers present in the department causing these faculty members to be less involved in the future direction of the department, and less invested in recruitment and retention (Griffith, 2010).

Career and work. Some women may find it difficult to reconcile future career goals that they perceive to require 12-16 hour days with future family planning goals that also require significant time commitments (Bystydzienski \& Brown, 2012; Szelenyi et al., 2013). Barriers such as internalized fear of failure, perceptions of tough grading scales in STEM (which may impact women's persistence more than men), and difficulty 
getting hands-on experience are other examples of how female students may not find STEM areas of study accessible (Griffith, 2010); however, barriers can also be as nuanced as the "chilly climates" of the classroom and beyond (Hall \& Sandler, 1982) and the lack of role congruity (Diekman et al., 2010). Students may be unaware of how gender inequality in their chosen field can influence their decisions to adjust their career goals and change their educational paths. Zeldin and Pajares (2000) explored the experiences of women in male-dominated career fields, specifically interviewing 15 female STEM professionals. Their goals included understanding various facets of the participants' self-efficacy, such as the sources of their self-efficacy, and the strategies they use to maintain their self-efficacy. The researchers hoped to capture their understanding of their own successful careers positioned in male-dominated fields. Although a contrasting approach was used, some similar goals to the approach of Zeldin and Pajares were sought by Jorgenson (2002), who explored the case that in maledominated fields such as engineering, awareness of gender constructs was often ignored, or considered unworthy of recognition or discussion; gender was not reported as a component contributing to the participants' understanding of their self-efficacy and success. These are just two of the many studies that highlight the many barriers listed thus far, and expand on the fact that these may not be the only reasons why women do not persist to leadership and decision-making positions at the same rate as men do in STEM fields of work.

Similar to the findings of Gonsalves (2014) in physics, interviews with 15 participants yielded stories about women in engineering fields who believed that by taking a non-gendered identity it became easier to take on male standards of success and 
styles of work (Jorgenson, 2002). Because of this positioning by many of the participants, they did not see themselves as "gender nonconformists in a male dominated field" as Jorgenson (2002) describes it. Furthermore, Jorgenson analyzed the narratives of the participants and revealed a series of connections to master narratives for women in male dominated fields. Among the common narratives shared by a number of participants, "engineering is a gender-neutral field" and, "positioning the self as a nonfeminist" (p.367) were reiterated consistently. Taking on these aspects of the master narratives of their field, and making them their own was seen as beneficial to them in their professional endeavors.

Across these studies, many of the participants expressed a learned interest and early understanding of their self-efficacy in math and science due to family members and teachers providing role modeling and in-depth support to them at an early age:

[I was] brainwashed by a high school physics teacher. I found that, if you were a female who was good in math and science, this particular teacher really believed in getting women into scientific degrees. So every year for 2 years that I was in his physics class, he said, "Marry a doctor, be an engineer." When I came to college and I was pre-med, I hated physics, though that is what I had planned to major in. Well, somewhere at this point, this saying kept going through my head...just marry a doctor and be a chemical engineer, and I went into chemical engineering. (Zeldin \& Pajares, 2000, p. 230)

Many of the participants expressed specific memories that influenced their decisions to believe that STEM was an acceptable path for them. And yet, many of them also expressed a distinct knowledge that they had forgone any other understanding of success through work other than the standard set by and for the male-dominated field they entered (Jorgenson, 2002): "Given that positions can be realized in intricate ways, occasionally the subject position not taken up by an individual is especially significant. 
Such choices can serve to mute or amplify the significance of a particular category such as gender in relation to other dimensions" (Jorgenson, 2002). In this way, Jorgenson used the data collected through the narratives to ask whether or not women who opt out of these fields are really opting out. It can be theorized that if there is room in STEM work spaces for limited variations of feminine gender identity consciousness, then the curriculum and policy changes affecting student persistence at earlier points on the pathway might be far more crucial than proposals to change those already in the STEM workforce.

\section{Summary}

New directions in research concerning the underrepresentation of women in STEM have been called for by many contributors to this discourse. For example, Perna et al. (2009) noted specifically that future research on women as STEM majors in college should focus on data collected from different sizes and types of institutions to enhance the depth and breadth of the information available related to institutional factors. This level of nuance and this attention to detail is what is required to adequately bring clarity to the complex, intersecting points of gender, race, ethnicity, and other identity concepts within STEM spaces. Therefore, it becomes pertinent to ask how higher education institutions can impact the direction of this discourse. Some higher education institutions have implemented support programs that are impacting persistence rates for women underrepresented in STEM majors while simultaneously providing an increasingly critical evaluation of the campus and program climates in which women must navigate both the academic and the cultural challenges inherent in these majors. In this study, analysis of data beyond the metrics of persistence will be bolstered by incorporating other 
conceptual frameworks such as physics identity, the "ideal" scientist, and gender role congruity, all of which expound on the complex nuances of the continued gender gap issue in some STEM fields (Johnson, 2012; Shapiro \& Sax, 2011).

This chapter presented a review of the literature concerning the STEM dilemma on inequality and underrepresentation by women, and the question, why diversify?

Women and STEM, and specifically, women and physics were also addressed, followed by an in-depth framing of the conceptual framework for this study within these contexts. The following chapter will cover the method proposed for this study. 


\section{CHAPTER III}

\section{METHODS}

\section{Restatement of the Problem}

As outlined in the first and second chapters, the literature examining the underrepresentation of women in math intensive STEM fields of study, such as physics, highlights the critical need to diversify within these fields. Given the fact that there is considerable attrition of students in these majors during college, it is important to learn more about how women persist in these majors and how they navigate these spaces. The purpose of this study was to further understand the experiences of women in physical science majors in higher education by examining the relationships between gender role congruity, physics identity, and beliefs about the "ideal" scientist with students' plans to persist in their chosen field. Employing an explanatory sequential mixed methods approach, quantitative data from students across the U.S. was collected to examine these primary relationships, while subsequent qualitative data was collected and utilized to explain the quantitative results. This study specifically focused on female undergraduates' physics identity (both as they see themselves and the "ideal"), when and how they understand gendered roles within their chosen field, and how they use this information to inform their decisions to persist in their major.

\section{Research Questions}

This study was framed by five research questions, three of which were explored during the quantitative strand and two of which were explored during the qualitative strand. The development of the qualitative data collection and analysis, and the subsequent mixed methods discussion were integrated to expand understanding across 
both strands. The participant population under investigation for this study and the data

collected were utilized to answer the research questions, which are as follows:

1. Quantitative: Is the way undergraduate women in physics see the "ideal" physics student related to their physics identity?

2. Quantitative: How does gender role congruity relate to physics identity?

3. Quantitative: What factors (i.e., physics identity, gender role congruity, perceptions of the "ideal" science student) relate to plans to persist?

4. Qualitative: How do the experiences of undergraduate women in physics majors delineate their perspectives related to their physics identity, the "ideal" physics identity, and gender role congruity within their chosen field of study?

5. Qualitative: How are the experiences of the participants similar, or different, based on the theoretical framework that guided this study?

\section{Research Design}

The explanatory sequential mixed methods design of this study involved collecting and analyzing quantitative data first and then utilizing in-depth qualitative data to further explain the findings.

As a result of the inherent weaknesses in isolated quantitative and qualitative approaches to research (Creswell, 2007, 2015), a mixed methods approach is appropriate in this case to address the complex nature of gender inequity in physical science fields of study. Creswell (2015) stated: "A core assumption of this approach is that when an investigator combines statistical trends with stories and personal experiences, this collective strength provides a better understanding of the research problem than either form of data alone" (p. 2). The complementary strengths of each approach allowed a look at a larger population for generalization and precision purposes while simultaneously maintaining the contextual voices of a sample of undergraduate women majoring in physical sciences. 


\section{Quantitative Strand}

In the first quantitative component of the study, survey data was collected by the American Physical Society (APS) from undergraduate students as part of the online registration process for the APS sponsored 2016 Conferences for Undergraduate Women in Physics (CUWiP). The survey included questions about gender roles and goals, behaviors of, and perceptions about, the "ideal" physics students, participants' own physics identity, and plans to persist in their chosen field. Other information was collected through the CUWiP survey, but only demographics and questions related to the four constructs focused on in this study were analyzed.

\section{CUWiP Data}

Since its inception in 2006, CUWiP takes place annually in January on the same dates at nine universities across the U.S. (see Appendix B for APS CUWiP 2016 Advertisement) involving approximately 1,000 participants each year. Host institutions have changed throughout the years, and universities interested in serving as a host site can submit applications to APS through their website. The locations for the 2016 conferences were: Black Hills State University; Georgia Institute of Technology; Ohio State University; Old Dominion University/Jefferson Lab; Oregon State University; University of California, San Diego; University of Texas, San Antonio; and, Wesleyan University. According to APS:

The primary goal of the Conferences for Undergraduate Women in Physics (CUWiP) is to increase recruitment and retention of undergraduate women in physics by:

1. communicating the breadth of education and career paths open to physics majors; 
2. disseminating information and advice on applying for summer research, graduate school and professional employment;

3. providing opportunities to share experiences, advice and ideas with women at different stages of their education or career paths. (www.aps.org, para.2)

Undergraduate students from universities located across the country attend the conference by first submitting an application through the host site at the location where they intend to attend CUWiP, and if selected, then they complete the online registration process and take the pre-conference survey. The survey was originally designed by a team of researchers on behalf of APS including external evaluators, Dr. Eric Brewe and Dr. Zahra Hazari of Florida International University, in 2013 to collect data from CUWiP participants in a pre- and post-conference format; to date, this is still the case. I was invited by Drs. Hazari and Brewe to design the survey questions for the 2016 PreConference CUWiP survey related specifically to the conceptual framework of the present study related to gender role congruity and perceptions of the "ideal" science student. Survey questions related to self-reported persistence plans and physics identity were already included in the survey based on iterations of the survey previously administered. The 2016 CUWiP pre-conference survey was distributed by APS in November, 2015, and was available to participants online until registration for the January, 2016 conference closed in December, 2015.

Examples of questions from the CUWiP survey that related to gender role congruity include: Do you believe that physics careers allow the fulfillment of the following goals? And, [h]ow important are each of the following kinds of goals to you personally? After each of these questions, the following list of gender role dimensions utilized by Diekman et al. (2010) to look at congruity between goals and roles for women 
in STEM careers is provided to the survey respondents for them to self-report the way

they see physics as a field that can fulfill these goals, as well as providing them an

opportunity to consider the way they personally value each of these goals (see Figure 5):

\begin{tabular}{|c|c|c|}
\hline & Not at all 01 & 234 Very much so \\
\hline Serving community & $\mathrm{OO}$ & $\mathrm{OOO}$ \\
\hline Working with people & $\mathrm{OO}$ & $\mathrm{OOO}$ \\
\hline Altruism (selflessness, self-sacrifice) & $\mathrm{OO}$ & $\mathrm{OOO}$ \\
\hline Helping others & $\mathrm{OO}$ & $\mathrm{OOO}$ \\
\hline Connecting with others & $\mathrm{OO}$ & $\mathrm{OOO}$ \\
\hline Serving humanity & $\mathrm{OO}$ & $\mathrm{OOO}$ \\
\hline Attending to others & $\mathrm{OO}$ & $\mathrm{OOO}$ \\
\hline Caring for others & $\mathrm{OO}$ & $\mathrm{OOO}$ \\
\hline Spirituality & $\mathrm{OO}$ & $\mathrm{OOO}$ \\
\hline Intimacy & $\mathrm{OO}$ & $\mathrm{OOO}$ \\
\hline Power & $\mathrm{OO}$ & $\mathrm{OOO}$ \\
\hline Recognition & $\mathrm{OO}$ & $\mathrm{OOO}$ \\
\hline Achievement & $\mathrm{OO}$ & $\mathrm{OOO}$ \\
\hline Status & $\mathrm{OO}$ & $\mathrm{O} \mathrm{OO}$ \\
\hline Focus on the self & $\mathrm{OO}$ & $\mathrm{OOO}$ \\
\hline Success & $\mathrm{OO}$ & $\mathrm{O} \mathrm{OO}$ \\
\hline Financial reward & $\mathrm{OO}$ & $\mathrm{OOO}$ \\
\hline Self-direction & $\mathrm{OO}$ & $\mathrm{OOO}$ \\
\hline Mastery (command of & $\mathrm{OO}$ & $\mathrm{OOO}$ \\
\hline knowledge/skills) & $\mathrm{OO}$ & $\mathrm{OOO}$ \\
\hline Self-promotion & $\mathrm{OO}$ & $\mathrm{OOO}$ \\
\hline Independence & $\mathrm{O} \mathrm{O}$ & $\mathrm{OOO}$ \\
\hline Individualism & $\mathrm{OO}$ & $\mathrm{O} \mathrm{OO}$ \\
\hline Demonstrating skill & $\mathrm{OO}$ & $\mathrm{OOO}$ \\
\hline Competition & $\mathrm{OO}$ & $\mathrm{OOO}$ \\
\hline
\end{tabular}

Figure 5. Gender roles/goals congruity CUWiP survey question. The questions related to these dimensions featured on the CUWiP 2015-2016 survey were adapted from "Seeking Congruity Between Goals and Roles: A New Look at Why Women Opt Out of Science, Technology, Engineering, and Mathematics Careers, " by A. B. Diekman, E. R. Brown, A. M. Johnston, and E. K. Clark, 2010, Psychological Science, 21 (8), p. 1054. Copyright 2010 by the Association for Psychological Science. Copyright for the CUWiP survey 2015 by the American Physical Society.

These questions were previously found reliable according to Diekman et al. (2010).

Previous factor analysis of the goal-endorsement items listed above revealed two distinct 
factors described as agentic goals and communal goals: “Cronbach's alphas indicate high internal consistency within each scale (agentic $(\alpha=.87)$ and communal $(\alpha=.84))$ " (p. 1054). CUWiP survey questions five and six addressed the dimensions of science identity and perceptions of the "ideal" science student (i.e., interest, recognition, performance, and competence), and questions one, two, and three ask the respondents to self-report their plans to persist in the field. For access to the complete 2016 CUWiP pre-conference survey, see Appendix C.

While most of the CUWiP survey questions were drawn from previously validated and reliable instruments developed for college students (Diekman et al.; Hazari et al., 2010), further reliability and validity testing of the items have been performed. To establish face and content validity, focus groups with 6 female undergraduate students were held by Drs. Brewe and Hazari to determine whether the questions are interpreted and responded to appropriately and whether the breadth of necessary content is included for the constructs. Content validity was further supported by the in-depth literature review provided here on the theorized constructs which contributed to the development of the questions specific to this study. Finally, construct validity was established through factor analysis of the constructs on CUWiP survey data from previous years. For reliability, this same data was used to calculate coefficient alpha for the constructs to ensure that alpha was well above the 0.7 recommended cutoff. Additional testing of the 2016 CUWiP data and variables pertinent to this study will be presented in Chapter 4 .

\section{Quantitative Sample}

The sample population consisted of 900 respondents who completed the registration survey to attend the $2016 \mathrm{CUWiP}$ in one of the nine locations. All 
respondents who self-identified as male or a gender other than female were removed;

therefore the sample consisted only of students who self-identified as female. Participants were attending universities located in a number of different areas across the U.S. The majority of participants were undergraduate students at the time when they took the survey; a few participants were graduate students. In addition, most of the students were physics majors with a few engineering majors also represented. Note that nationally only 1,162 women graduated with bachelor's degrees in physics in 2015 (NSB, 2016). Thus, the CUWiP data represents a large fraction of the women undergraduates in physics.

\section{Quantitative Data Analysis}

After the survey results were collected, the data was input into R, which is a programming language and software environment for statistical computing and graphics outputs. A series of tests were run on the data which included linear regression and multiple linear regression analysis to determine if relationships exist between the students' understanding of the "ideal" student in their field and how they perceive their own identity in physics, as well as to understand if there is a relationship between their perceived gender role(s) and identity. A multiple regression was run to determine how the three independent variables ("ideal" scientist, physics identity, gender role congruity) relate to the dependent variable (plans to persist). Collinearity issues were addressed by checking variance inflation factors on all regressions with more than one independent variable and were found to be below 2 in all cases (ranging from 1.04-1.28).

In addition to the analysis of the CUWiP data to answer the quantitative research questions of this study, responses to CUWiP questions related to the conceptual framework were scored to facilitate the stratification of participants in relation to the 
conceptual framework. For example, the third CUWiP survey question asks: Do you intend to complete a bachelor's degree in physics? The Likert scale response options are: Not at all 01234 Very much so. Responses of 0 and 1 were scored as "Low Bachelor's Degree Persistence Plans." The score was indicated in the database with an "LB." Responses of 2 were labeled as "Mid Bachelor's Degree Persistence Plans" and marked in the data base with “ ." Responses of 3 and 4 were labeled "High Bachelor's Degree Persistence Plans" indicated by "HB" in the database. Scoring responses in this way was repeated for graduate school plans $(\mathrm{Q} 2)$, career plans $(\mathrm{Q} 1)$, physics identity and the “ideal” science student (Q5 and Q6), and gender role congruity dimensions (Q19), and the same labels were applied to all 900 quantitative sample participants. For questions with multiple variables making up the concept, the responses for all related variables were averaged to determine each participant's score. For example, all scores for variables related to the communal gender roles and goals (Q19) were averaged to determine whether their overall communal gender roles/goals score was low, medium, or high. I decided it would be easier to make decisions about who to invite to participate in the qualitative phase of the study if I had as much information about participants' scores on these variables as possible. Therefore, I further stratified scores on questions measuring multiple variables by indicating "very high" and "very low" scores. Therefore a "very high" score on communal gender roles/goals meant that all of the participant's responses averaged to the highest score available to them, "4." A "very low" score indicated that the average of their responses to all of the communal variables fell below 1 . A "low" score was assigned to anyone whose average of their responses to the communal variables was between 1 and 1.99. A "mid" score indicated by " " in the database 
represented an average of their responses equaling 2-2.99. Finally, a score of "high" was assigned to every participant whose average responses to the communal items was between 3 and 3.99. The same score system was then applied to all other variables of the questions related to the conceptual framework (e.g., physics identity, the "ideal" science student, extrinsic agentic gender roles/goals, and intrinsic agentic gender roles/goals). Figure 6 lists all of the scores and their corresponding tags that were inserted into the database next to the respondent's name and demographic responses: 
2016 CUWiP Data Score Key

HB High Bachelor's Degree Persistence Plans (Q3) (scores above 3)

LB Low Bachelor's Degree Persistence Plans (Q3) (scores below 2)

HG High Graduate Persistence Plans (Q2) (scores above 3)

LG Low Graduate Persistence Plans (Q2) (scores below 2)

*Hca Very High Career Persistence Plans (Q1ABCD) (Score of 4 on all items)

Hca High Career Persistence Plans (Q1ABCD) (average between 3-3.99)

Lca Low Career Persistence Plans (Q1ABCD) (average between 1-1.99)

*Lca Very Low Career Persistence Plans (Q1ABCD) (average below 1)

HID High Identity (Q5 \& 6)

LID Low Identity (Q5 \& 6)

HIS High "Ideal" Scientist (Q5 \& 6)

LIS Low "Ideal" Scientist (Q5 \& 6)

*HC Very High Communal (Q19) (average communal score of 4)

HC High Communal (Q19) (average communal score between 3-3.99)

LC Low Communal (Q19) (average communal score between 1-1.99)

*LC Very Low Communal (Q19) (average of communal variables below 1)

*HAE Very High Agentic-Extrinsic (Q19) (average extrinsic agentic score of 4)

HAE High Agentic - Extrinsic (Q19) (average extrinsic agentic score between 3-3.99)

LAE Low Agentic - Extrinsic (Q19) (average extrinsic agentic score between 1-1.99)

*LAE Very Low Agentic - Extrinsic (Q19) (average extrinsic agentic score below 1)

*HAI Very High Agentic - Intrinsic (Q19) (average of intrinsic agentic variables equaled the highest score of 4)

HAI High Agentic - Intrinsic (Q19) (average intrinsic agentic score between 3-3.99)

LAI Low Agentic - Intrinsic (Q19) (average intrinsic agentic score between 1-1.99)

*LAI Very Low Agentic - Intrinsic (Q19) (average intrinsic agentic score below 1)

This symbol represents an average of scores in any of the above categories $\sim$ between 2-2.99e

Figure 6. Scores and corresponding tags associated with participants' responses to the CUWiP survey questions related to the conceptual framework of this study.

What the scoring and tagging system allowed me to do was then see a visual representation of all participants' responses to the CUWiP survey questions specific to the present study at a glance. Table 1 (below) exemplifies the list of scores on the CUWiP survey questions specific to the conceptual framework for this study as 
represented by the tags for the $18 \mathrm{CUWiP}$ participants who participated in the qualitative phase of this study:

Table 1

Qualitative Strand Participants' CUWiP Survey Scores

\begin{tabular}{|c|c|c|c|c|c|c|c|c|}
\hline $\begin{array}{l}\text { Qualitative } \\
\text { Participants }\end{array}$ & $\begin{array}{l}\text { Bach } \\
\text { Pers }\end{array}$ & $\begin{array}{l}\text { Grad } \\
\text { Pers }\end{array}$ & $\begin{array}{c}\text { Career } \\
\text { Pers }\end{array}$ & Identity & $\begin{array}{c}\text { "Ideal" } \\
\text { Scientist }\end{array}$ & $\begin{array}{l}\text { Com- } \\
\text { munal }\end{array}$ & $\begin{array}{l}\text { Extrinsic } \\
\text { Agentic }\end{array}$ & $\begin{array}{l}\text { Intrinsic } \\
\text { Agentic }\end{array}$ \\
\hline Participant 1 & HB & $\mathrm{HG}$ & HCA & HID & HIS & $\sim \sim$ & HAE & *HAI \\
\hline Participant 2 & HB & $\mathrm{HG}$ & $\mathrm{HCA}$ & HID & HIS & $\mathrm{HC}$ & $\sim \sim$ & HAI \\
\hline Participant 3 & HB & $\mathrm{HG}$ & $\sim \sim$ & $\sim \sim$ & HIS & $\sim \sim$ & $\sim \sim$ & $\sim \sim$ \\
\hline Participant 4 & $\mathrm{HB}$ & $\mathrm{HG}$ & $\mathrm{HCA}$ & HID & $* \mathrm{HIS}$ & $* \mathrm{HC}$ & $* \mathrm{HAE}$ & $* \mathrm{HAI}$ \\
\hline Participant 5 & $\mathrm{HB}$ & $\mathrm{HG}$ & $\sim \sim$ & HID & $\sim \sim$ & $\sim \sim$ & $\sim \sim$ & HAI \\
\hline Participant 6 & $\mathrm{HB}$ & $\mathrm{HG}$ & $\sim \sim$ & HID & HIS & $*^{2} \mathrm{LC}$ & $\sim \sim$ & *HAI \\
\hline Participant 7 & HB & $\mathrm{HG}$ & LCA & HID & HIS & $\sim \sim$ & HAE & $* \mathrm{HAI}$ \\
\hline Participant 8 & HB & $\mathrm{HG}$ & $\sim \sim$ & LID & *HIS & $\mathrm{LC}$ & $\sim \sim$ & $\sim \sim$ \\
\hline Participant 9 & HB & $\sim \sim$ & $\mathrm{HCA}$ & HID & HIS & $* \mathrm{LC}$ & LAE & HAI \\
\hline Participant 10 & HB & $\mathrm{HG}$ & $\sim \sim$ & HID & HIS & $* \mathrm{HC}$ & $* \mathrm{LAE}$ & LAI \\
\hline Participant 11 & $\mathrm{HB}$ & $\mathrm{HG}$ & $\sim \sim$ & $\sim \sim$ & $* \mathrm{HIS}$ & $\mathrm{HC}$ & $*$ LAE & LAI \\
\hline Participant 12 & $\mathrm{HB}$ & $\mathrm{HG}$ & $\sim \sim$ & HID & HIS & $\mathrm{HC}$ & HAE & HAI \\
\hline Participant 13 & HB & $\mathrm{HG}$ & $* \mathrm{HCA}$ & HID & *HIS & $\mathrm{HC}$ & LAE & HAI \\
\hline Participant 14 & HB & $\mathrm{HG}$ & $\mathrm{HCA}$ & HID & HIS & $\sim \sim$ & $\sim \sim$ & HAI \\
\hline Participant 15 & HB & LG & $\sim \sim$ & LID & HIS & $\sim \sim$ & LAE & $\sim \sim$ \\
\hline Participant 16 & HB & $\mathrm{HG}$ & $\sim \sim$ & HID & *HIS & $* \mathrm{HC}$ & $* \mathrm{HAE}$ & *HAI \\
\hline Participant 17 & HB & $\mathrm{HG}$ & $* \mathrm{HCA}$ & $\sim \sim$ & HIS & $\mathrm{HC}$ & LAE & $\sim \sim$ \\
\hline Participant 18 & $\mathrm{HB}$ & $\mathrm{HG}$ & $\sim \sim$ & $\sim \sim$ & HIS & $\mathrm{HC}$ & $\sim \sim$ & HAI \\
\hline
\end{tabular}

Chapter 4 provides additional details for the quantitative data analysis findings of this study.

\section{Qualitative Strand}

The second, qualitative strand was conducted as a follow-up to the quantitative results to help further explain the quantitative findings. Utilizing demographics, progress in their physics major (i.e., year in school and total number of physics courses completed as reported on the survey), GPA, and their scores on the variables related to conceptual 
framework as selection criteria, qualitative participants were purposefully selected to facilitate and expand understanding of the quantitative findings (Bogdan \& Biklen, 2007; Creswell, 2015; Seidman, 2013). Only participants from the data set utilized in the quantitative strand were contacted to participate in the qualitative strand. The qualitative phase of the study was used to explore participants' perceptions and understanding of gender role congruity, physics identity, and their perceptions and assumptions about the "ideal" expectations in their major. Furthermore, qualitative were was utilized to learn how these students articulate relationships between these factors when discussing their academic and career persistence plans. A semistructured interview protocol was developed with questions derivative of the qualitative research questions and the various dimensions of the conceptual framework (Rubin \& Rubin, 2012). The interview protocol was also developed with the results of the quantitative analysis in consideration with the goal being that these questions would further explore and explain the quantitative findings. For a complete list of the interview protocol questions, please see Appendix D. The semistructured interviews were conducted over the phone (and one via Skype) utilizing a general interview guide approach (Gall, Gall, \& Borg, 2003; McNamara, 2008; Turner, 2010), which allowed the research questions and protocol to provide considerable structure while simultaneously offering flexibility to collect data from each interviewee in the manner most appropriate to the direction and flow of the interview.

\section{Qualitative Sample}

In mixed methods research, Creswell (2014) suggests planning and choosing qualitative participants carefully, and that these decisions can be based on any number of opportunities such as: "extreme or outlier cases, significant predictors, significant results 
relating variables, insignificant results, or even demographics" (p. 224). Sample selection criteria for this strand of the study included capturing variation in the demographic data, representative cases of the relational factors among the four constructs under investigation for this study, and extreme or outlier cases. The sample for this phase of the study consisted of 18 participants. This number of participants was sufficient because although I was prepared to add additional participants, the data saturated at this point (i.e., I noticed repeating trends across the data such as similarities in experiences as female physics majors, as well as differences that aligned with the conceptual framework that guided the present study) (Akerlind, 2012; Marton \& Booth, 1997). Additionally, according to Creswell (1998), and Tashakkori \& Teddlie (2011), qualitative sample sizes typically range from six to 25 participants. All 18 participants met the sampling criteria.

Specifically considering demographic criteria, a number of CUWiP survey items were used to create a list of participants to invite to participate in this strand of the study. First, all participants who reported being in their third, fourth, or fifth year of their undergraduate degree were prioritized in order to collect data from students who had most likely had opportunities to experience some or all of the aspects of the conceptual framework. The list of participants who had completed their first and second undergraduate year was set aside as a backup in the event that not enough participants meeting this criteria volunteered to participate; however, this was not necessary as everyone who completed interviews met this criterion. As a consequence of the fact that the common practice of undergraduate physics majors engaging in research experiences either at their university, through the National Science Foundation's (NSF) Research 
Experiences for Undergraduates (REU), or through other organizations, seeking participants who were further along in their studies likely meant that they had already had opportunities to engage in research which served as an experience to examine with respect to the conceptual framework. Identifying students in their third, fourth, or fifth year was also determined to be a sufficient threshold because then participants would have likely gone beyond merely declaring a physics major and had completed courses in their major, thus serving as additional experiences to examine. All 18 qualitative participants were in their third or fourth year of their undergraduate work.

Other criteria used to achieve similar goals of speaking with students who had already had opportunities to experience the academic work and culture of their physics majors was that their major was a physical science, and that they had taken physics courses (i.e., CUWiP survey asks the respondent to self-report their major and the number of physics courses they completed in college out of nine common options e.g., Intro Physics I, Modern Physics, Quantum Mechanics I, etc. (see Appendix C, Q15 for the complete list)). All 18 qualitative participants had completed a minimum of two physics courses from the list, with the average being 5.2 physics courses completed. All 18 qualitative participants were physics or physics related majors, with more than half of the sample also having a second major or minor (only one participant in the qualitative sample had a second major in an area of study outside of STEM).

A GPA of 3.0 or higher was initially assumed to be a useful criterion for qualitative sample selection. This was due to an assumption that GPA would positively (or negatively) predict plans to persist. However, a test was run including GPA to determine if this would be an acceptable criterion to include but no significant effect was 
observed for GPA on plans to persist for the CUWiP sample. Therefore, GPA was not included in the selection process for qualitative participants.

Finally, race and ethnicity categories were prioritized and carefully considered when selecting those who would receive an invitation to participate in this phase of the study. A preponderance of studies in the past have been conducted with entirely White samples, which in turn has made it challenging, if not impossible, to apply the findings to the experiences of students in other racial and ethnic identity groups. These perspectives are important because prior work has revealed additional experiential factors for these groups such as racial prejudice, discrimination, and systemic oppression (Espinosa, 2011). Additionally, the NSF has identified Asians as represented at a higher rate in STEM than in the population (NSF, 2015); therefore, all female CUWiP respondents who reported a racial or ethnic identity other than White and Asian (i.e., Hispanic, Latina, Black, Native American or Alaskan Native, Native Hawaiian or Pacific Islander, or "other") and who met the above criteria, were included in the first round of invitations to participate in the study.

A list of participants meeting all of the above criteria was created to extend invitations to participate. A total of 119 participants made up this list (13 Black, 10 Native American or Alaskan Native (NAAN), 7 Native Hawaiian or Pacific Islander (NHPI), 15 Other, and 74 Hispanic). The invitation was distributed to the email addresses provided by the participants when they completed the CUWiP survey. This message introduced me as the researcher, explained that I was inviting their participation because they had completed the CUWiP survey, and explained what I was asking of them, including their total estimated time commitment. Additionally, the FIU IRB approval 
number and contact information for Dr. Hazari was provided. Mail merge, a Microsoft Word tool which allows for the automatic addition of information from a database to an email message, was utilized to personalize the email message greeting line (e.g., Dear Dakota, or Dear Gabriella, etc.). Additionally, Starbucks gift cards were offered as incentive to students to participate in this strand of the study. Participants were promised \$20 in Starbucks gift cards for completing the interview with me. For the complete text of the email invitation to participate in this phase of the study, please see Appendix F. Chapter 6 provides a discussion on the limitation of utilizing the gift cards as incentive.

After an adequate amount of time was given to respond to the invitation, a second round of invitations was sent to 134 White and Asian participants. Because there were many more than 134 White and Asian participants in the CUWiP data set, additional criteria was used to determine who would receive an invitation such as geographic location, institution type (e.g., public, private, large, small, etc.), and diversity in their responses to the CUWiP questions specific to this study. The CUWiP scores (e.g., $* \mathrm{HC}$, LID, etc.) allowed me to look for diversity opportunities, and even to target missing scores. For example, I had received responses from participants whose scores across the gender roles/goals variables were all "high" or "very high." Therefore I extended invitations to participants with "low" and "very low" communal scores, and/or "low" and "very low" agentic scores. I noticed that I had few or no participants with either "low" physics identity scores, or a combination of scores representing "high" or "very high" extrinsic agentic goals and "low" or "very low" intrinsic agentic goals. I was able to then follow up with participants with these scores who had responded, or identify additional participants to whom I extended invitations. 
Therefore, after contacting a total of 253 participants from the quantitative sample who met the criteria for the qualitative strand in the manner outlined above, a total of 60 responses were received for a response rate of $23.7 \%$. With a higher number of responses than the 15-20 goal originally proposed, participants who responded to my initial email invitation and represented diversity in the conceptual framework and other criteria discussed above were sent a follow up email either requesting to schedule an interview, or a notification that a high volume of responses an interview may be scheduled at a later date if needed.

\section{Qualitative Data Collection}

As detailed in the previous section, women who met the qualitative strand selection criteria were contacted via the email address they provide when they registered for CUWiP and invited to interview individually for 60 minutes. Because of the importance of the opportunity to ask probing questions in qualitative research afforded by an interview with a participant (Bogdan \& Biklen, 2007; Rubin \& Rubin, 2012; Seidman, 2013), and taking the location of the students who participated in this strand of the study into consideration (students were spread across the U.S. since the locations of the CUWiP are spread across the U.S.), a total of 18 interviews averaging 60 minutes in length were conducted, 17 of which were conducted by phone, and one interview was conducted through Skype. An additional four participants were scheduled for interviews but did not complete the interview or follow up further. All interviews were audio recorded using an iPhone application called, Call Recorder, which allows the call to be recorded while the phone is in use, a feature not available through the iPhone by default. The audio recordings were then transferred to the Rev.com app and transcripts of the recorded 
interviews were obtained. When the completed transcripts were received from Rev, I reviewed each one thoroughly to make edits and check if any "inaudible" points could be clarified by comparing to the original recording of the interview. After it was determined that sufficient data was collected from the 18 interviews, the 18 participants interviewed were contacted two additional times each, once by email with a copy of the transcript for their review and feedback, and once by United States Postal Service mail to provide them with a thank you note and the Starbucks gift cards promised in the original email invitation. For the complete text of this follow up email, please see Appendix G. Two participants sent feedback on their transcripts, and three confirmed that they approved the transcript without edits.

The qualitative data collected provided many examples of students' experiences as well as thought and language patterns that participants utilized to express their perspective (Creswell, 2014; Seidman, 2013; Van Manen, 1990).

\section{Qualitative Data Analysis}

Using the transcripts of the interviews, a Computer Assisted Qualitative Data Analysis System (CAQDAS), called MAXQDA, was utilized to code the interviews to identify attributes, significant ideas, and essence-capturing language (Saldaña, 2013). The first round of coding employed a number of coding methods including the simultaneous coding method (Miles \& Huberman, 1994) to take advantage of the strengths of both structural coding based on the conceptual framework (MacQueen, McLellan, Bartholow, \& Milstein, 2008; Saldaña, 2013) and initial coding opportunities (Charmaz, 2006). These coding methods were utilized to capture participants' experiences and perspectives about other related concepts typical to this line of inquiry (e.g., chilly climate, 
mentorship, discrimination, etc.). Coding the transcripts this way provided the opportunity for open-ended aspects of the latter approach to be used to capture related experiences emergent in the data. Therefore, although an initial set of codes based on the conceptual framework was utilized from the beginning of the coding process, the list of codes was not considered complete until all 18 transcripts had undergone the first round of coding and the emergent codes were revealed. A total of 51 codes were identified throughout this analysis. Codes were assigned to passages simultaneously, meaning that however many codes were deemed appropriate for a passage they were assigned to the passage simultaneously. An effort was made to be parsimonious when assigning multiple codes to a passage which might therefore have one code, or might have multiple codes (e.g., in a few cases passages were assigned over 10 codes). Figure 7 provides a visual of the code system in MAXQDA with the Physics Identity code expanded to show the subcodes titled according to the four dimensions of the physics identity framework:

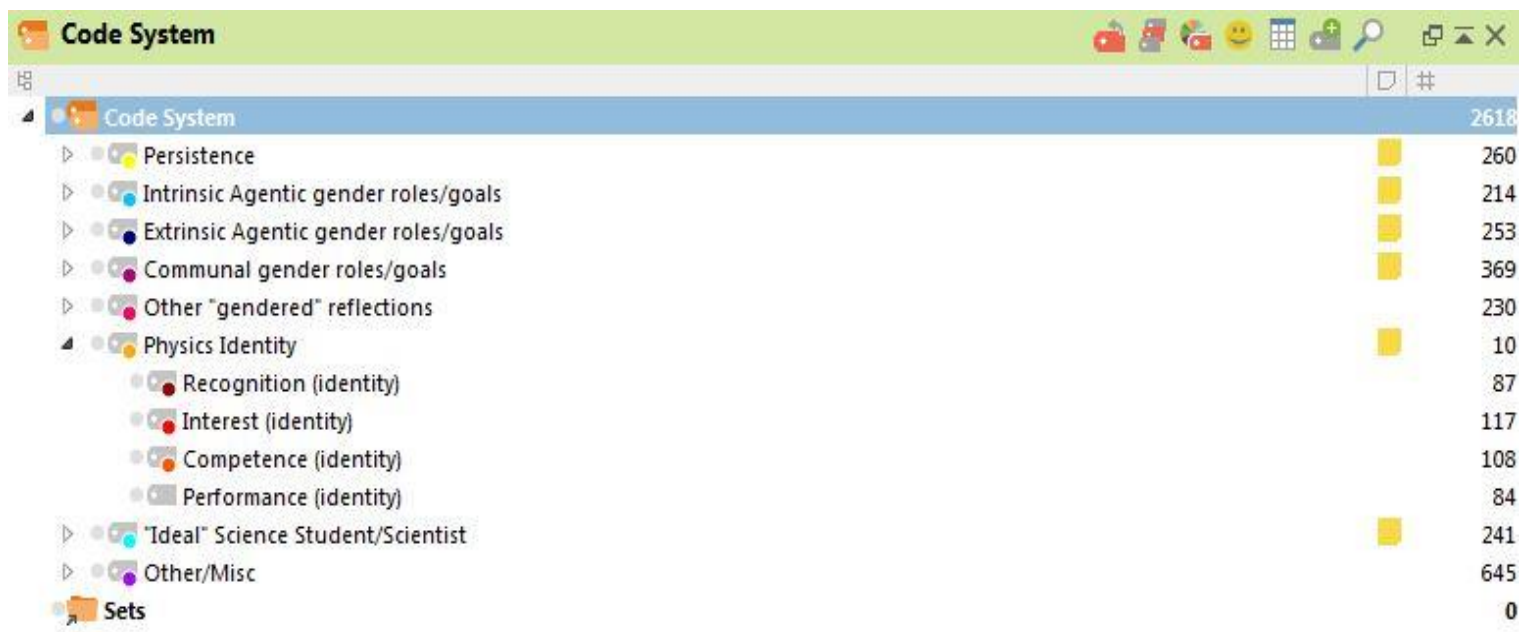

Figure 7. Overview of the code system as displayed in MAXQDA.

For additional visuals providing expanded views of the subcodes underneath all codes listed above, please see Appendix H. In addition to codes that represent the conceptual 
framework, and the codes identified through initial coding, passages that seemed to be typical and atypical exemplars of the conceptual framework were noted at this stage (Saldaña, 2013).

Following this first round of coding, a phenomenographic approach was utilized to conduct the remaining qualitative analysis cycles. The complex coding query feature of MAXQDA allowed me to pull passages coded with specific combinations of codes and subcodes. For example, passages coded with at least one persistence code or subcode and at least one communal gender role/goal code or subcode were examined in a constant comparison fashion (Glaser, 1965) looking for opportunities to consolidate the codes assigned to the passages based on the depth and breadth of the differences and similarities of passages that were assigned those overlapping codes during the first round.

Throughout this process I was coding and categorizing the qualitatively different ways the participants were experiencing the nuances of the phenomena that make up the conceptual framework. I was also identifying the criteria that caused different passages to be placed in one category or another, with many cycles of testing the criteria as recommended by Marton $(1981,1986)$. Initially I attempted to present these categories according to whether or not the participants had articulated plans to persist in a physics field beyond their bachelor's degree or not, or if they articulated an "undecided" plan. However, after extensive effort to delineate the phenomenographic categories of understanding from these three perspectives, a number of categories of understanding were not able to withstand the rigorous and constant "testing" resulting in an inability to adequately condense the information in to the most sparing number of categories, as Marton and Booth (1997) recommend. 
Therefore, an additional round of focused coding analysis (Charmaz, 2006) was conducted by reviewing all transcripts again, seeking to reframe the categories that represented the qualitative differences across the data based on the underlying meaning of each transcript as a whole (Akerlind, 2012). I noted characteristics, participant CUWiP scores, and the most sparing description of how the participant was positioned in relation to the conceptual framework. Once I had this brief profile describing the underlying meaning of the whole transcript, I then grouped coded segments of participant's common relationships with the framework. For example, I would examine coded segments of all participants with similar descriptions of the dimensions of their physics identity, or shared reflections and experiences about gender or assumptions about the "ideal" stereotypes. These segments were then coded by relationship to the conceptual framework the participants were describing (i.e., high or low competency beliefs). The depths of the similarities and differences between these shared relationships were analyzed in a cyclical fashion. At each stage of grouping concepts, experiences, relationships, and finally participants, ongoing discussions and review of the data were held weekly with the principle investigator. Ultimately, the qualitatively different ways the participants experience their relationships with the conceptual framework formed five groups of participants, called categories of experience, detailed in Chapter 5.

Marton and Booth (1997) elaborate on what they call the phenomenographic outcome space with three distinct criteria to evaluate the results: 1) distinctive categories presenting unique relationships with the phenomena as experienced by the participants; 2) these distinct categories are presented as they logically relate to each other, "frequently hierarchical;" and, 3) the fewest number of categories possible are used to represent the 
data (p. 125). These criteria were utilized to discuss the quality of the outcome space presented in Chapter 5.

\section{Reliability and Reflexivity}

Chase (2005) cautioned qualitative researchers: “[A] central question revolves around which voice or voices researchers should use as they interpret and represent the voices of those they study" (p. 652). For example, because I personally advocate for gender equity daily through my job at FIU as the Director of the Women's Center in the Division of Student Affairs, I had to make a sincere effort to utilize member checking and triangulation (Merriam, 2014) to navigate the process of presenting the information with as little bias as possible. In addition to sharing the transcripts with participants providing them an opportunity to verify that their words have been recorded correctly, the multiplicity inherent in the guiding conceptual framework, the mixed methods approach, and the phenomenographic approach to the qualitative analysis provided many checkpoints and opportunities to audit whether or not I was representing what the participants said collectively, rather than merely my interpretation or privileging of what any one participant said individually (Denzin, 1997). Although one of the goals of my study was to bring awareness and consciousness to a critical equity issue in higher education, the goal was not to manipulate or privilege any data that confirmed the equity argument because my beliefs were confirmed. In fact, bias of this nature within the study could have discredited the voices of the qualitative participants, or discredited my results (Denzin \& Lincoln, 2003). Therefore, it was important that I always kept the authoritative nature of my own voice in this context at the fore of my reflections so as not to influence the validity of the study. All of these practices contributed significantly to constructing 
trustworthiness and validity for the qualitative portion (Clandinin \& Connelly, 2000; Merriam, 2014; Tracy, 2010; Trahar, 2009).

\section{Mixed Methods Data Integration}

With these goals in mind, the qualitative strand of the study was designed to gather data that would further explain the quantitative results of the study; the qualitative results also highlighted the voices and experiences of underrepresented women majoring in physics in the U.S. The integration of the two data sets and findings to further understand the results of the first phase of the study will be presented in Chapter 6 .

\section{Summary}

To achieve the goals of this study, I have chosen an explanatory sequential mixed methods design, the purpose of which is for the quantitative strand to frame the qualitative strand, and the qualitative phase to in turn explain the quantitative results further. This type of study will be beneficial to those interested in understanding whether relationships exist between these complex concepts for women engaging in math intensive, male-dominated fields of study in higher education and how they are exemplified and explained by the lived experiences of the women. 


\section{CHAPTER IV \\ QUANTITATIVE RESULTS}

As discussed in previous chapters, the purpose of this study was to examine the relationships between the constructs of gender role congruity, physics identity, the “ideal" physics student, and plans to persist in a physics or engineering major for undergraduate female students via an explanatory sequential mixed methods design. The quantitative results of this study are presented in this chapter for the purpose of providing higher education researchers and practitioners with findings that may be useful when considering the retention and graduation rates of undergraduate female students engaging in STEM majors that are persistently male-dominated, specifically, the physical sciences. The increased emphasis for faculty and administrators alike to commit to, and engage in, practices that increase diversity along the STEM pathway is supported by the results of my study, which aimed to further illuminate the complexities contributing to the gender gap in physics, rather than to prescriptively provide a road map for closing this gap. With this information, higher education institutions can further their understanding of their role in broadening the participation of women in particular STEM fields.

This chapter presents the quantitative results of the study and is organized into three major sections followed by a summary: sample demographics, construct validity and reliability, and the findings.

\section{Quantitative Strand}

Survey data was collected online by the American Physical Society (APS) from college students across the U.S. who registered for the 2016 annual Conferences for Women in Physics (CUWiP), which took place in nine locations across the country and 
was sponsored by the APS. The survey items used for constructs studied in this work were described previously in Chapter 3. The purpose of analyzing this data was to further understand the relationships between the constructs, thereby addressing the research questions for this phase of the study.

\section{Sample Population Demographics}

A total of 1338 students submitted applications to APS to attend the 2016 CUWiP. APS then distributed the online pre-conference survey to those students who were accepted as part of their registration process, resulting in 953 students who completed the pre-survey.

\section{Gender}

Of the 953 student respondents to the 2016 CUWiP pre-survey, $94.4 \%(n=900)$ of the sample identified as female, $3.3 \%(n=31)$ of the sample identified as male, and $<1 \%(n=5)$ of the sample identified as a gender other than female or male. A total of $1.8 \%$ did not respond to this question $(n=17)$. For the remainder of the sample demographics presented, participants of all genders were included in the summaries because the differences in demographic distributions when including all genders or only females were nominal. However, for the GPA and year in school overview (because these were both criteria for choosing participants for the qualitative phase of the study and used in subsequent statistical analysis described in this chapter), the factor analysis and all subsequent statistical analyses described in this chapter, data from only students who identified as female was used (see R code in Appendix I cuwip16 vs. cuwip16f). 


\section{Race/Ethnicity}

A frequency analysis of race and ethnicity indicated CUWiP participants' race and ethnicity were reported in the following breakdown: $15.3 \%$ Hispanic $(n=146), 4.1 \%$ Black $(n=39), 76.2 \%$ White $(n=726), 16.5 \%$ Asian $(n=157), 2 \%$ Native American or Alaskan Native $(n=19),<1 \%$ Native Hawaiian or Pacific Islander $(n=9)$, and $2.6 \%(n=$ 25) reported other, which included write-ins such as mixed-race, mixed-ethnicity, specific indigenous groups not listed, specific ethnicities not listed, and nationalities from around the world. Furthermore, because the CUWiP survey allowed respondents to "mark all that apply" to the list of races provided (i.e., Black, White, Asian, Native American or Alaskan Native, Native Hawaiian or Pacific Islander) 17\% $(n=162)$ respondents utilized this option, thereby reporting a mixed race/ethnicity.

\section{Year in College}

Utilizing a frequency analysis of the year in college of CUWiP participants, $12.1 \%(n=115)$ of respondents reported being in their first year of their undergraduate degree, $20.1 \%(n=192)$ in their second year, 30.7\% $(n=293)$ in their third year, $23.6 \%$ $(n=225)$ in their fourth year, 6.7\% $(n=64)$ in their fifth year, and <1\% $(n=2)$ reported being a graduate student. A total of $6.5 \%(n=62)$ of respondents left this question blank. For a complete summary of the sample population demographics, see Table 2 below: 
Table 2

Sample Population Demographics

\begin{tabular}{|c|c|c|}
\hline Demographic & Frequency & Percentage \\
\hline Female & 900 & 94.4 \\
\hline Male & 31 & 3.3 \\
\hline Other (Gender) & 5 & $<1$ \\
\hline Hispanic & 146 & 15.5 \\
\hline Black & 39 & 4.1 \\
\hline White & 726 & 76.2 \\
\hline Asian & 157 & 16.5 \\
\hline Native American or Alaska Native & 19 & 2 \\
\hline Native Hawaiian or Pacific Islander & 9 & $<1$ \\
\hline Other (Race/Ethnicity) & 25 & 2.6 \\
\hline Mixed Race/Ethnicity & 162 & 17 \\
\hline First Year & 124 & 13 \\
\hline Second Year & 198 & 20.7 \\
\hline Third Year & 306 & 32.1 \\
\hline Fourth Year & 233 & 24.4 \\
\hline Fifth Year & 69 & 7.2 \\
\hline Graduate Student & 2 & $<1 \%$ \\
\hline
\end{tabular}

\section{Physics Courses Taken}

CUWiP participants were asked to report the physics courses they have taken and completed in college; they were given the option to mark all that apply from the following list: Intro Physics I, Intro Physics II, Modern Physics, Classical Mechanics (not intro), Thermodynamics (Stat. Mech.), Electromagnetism I, Electromagnetism II, Quantum Mechanics I, Quantum Mechanics II. Approximately 5\% of the sample $(n=48)$ reported taking and completing no physics courses in college, and less than $1 \%$ of the sample $(n=9)$ did not answer this question. Because the remaining demographic data was used to identify qualitative phase participants, all remaining results are based on the data set with the male and other participants removed. Figure 8 provides a histogram view of the number of physics courses taken: 


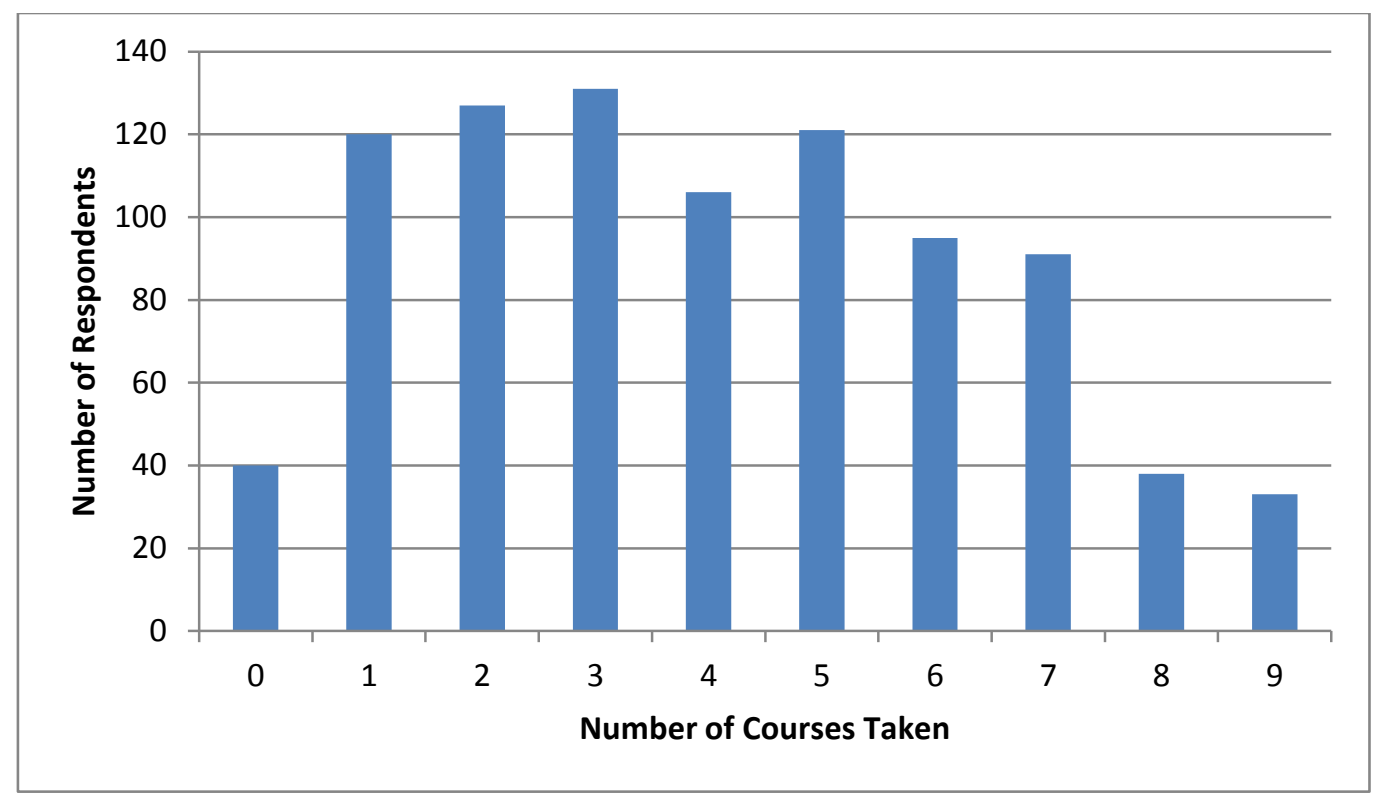

Figure 8. Histogram of the number of physics courses completed by CUWiP respondents.

\section{GPA}

CUWiP participants reported their approximate grade point average (GPA) in the physics courses listed above based on the following scale: $4.0=\mathrm{A}, 3.0=\mathrm{B}, 2.0=\mathrm{C}, 1.0=\mathrm{D}$, $0=\mathrm{F}$; they were given the opportunity to report this number up to two decimal points. The mean GPA was 3.4 with a standard deviation of 0.6 .

\section{Construct Validity and Reliability}

The data was first analyzed using a factor analysis to determine the construct validity of the measures for this population (Osborne, Costello, \& Kellow, 2008). The internal consistency reliability was then tested for the items in each construct.

\section{Gender Role Congruity}

Figure 9 displays the survey question CUWiP participants answered related to gender roles and goals, and which items were removed after loading below the 0.4 cutoff (Tabachnick \& Fidell, 2007): 


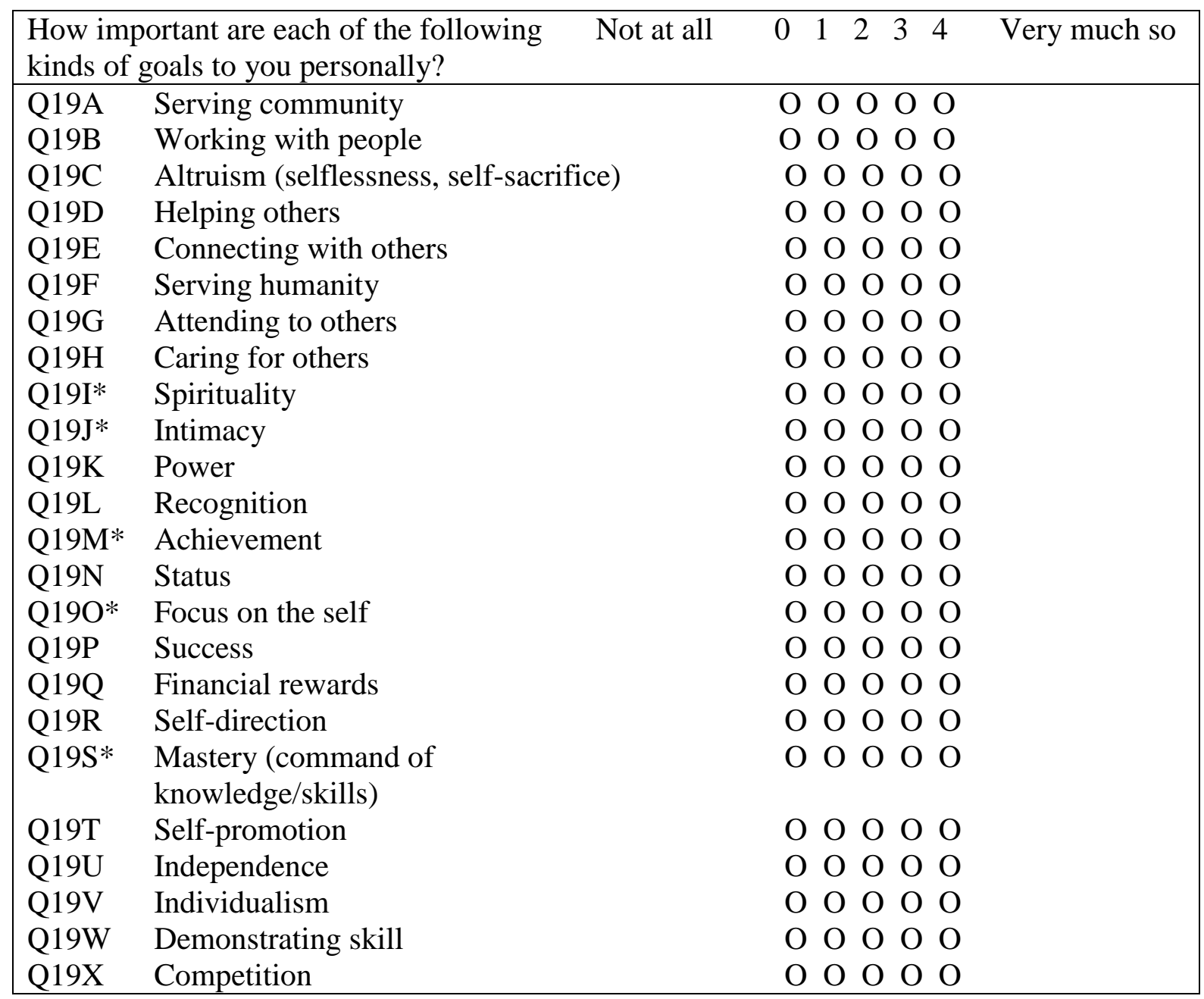

Figure 9. CUWiP question 19: Gender roles/goals. How important are each of the following kinds of goals to you personally?

*indicates item was removed after loading below the 0.4 cutoff during the factor analysis

I initially ran the factor analysis on these constructs and oblique promax rotation allowing the factors to correlate as a two factor model to align to the theoretical framing of

Diekman et al. (2010, 2017), representing the communal and the selected agentic factors.

However, since a high number of ostensibly related agentic dimensions of the gender role congruity theory were loading too low, further analysis using a scree plot and parallel 
analysis were utilized to determine the factors necessary to include in the study (Tabachnick \& Fidell, 2007). Scree test and plot results are featured in Figure 10:

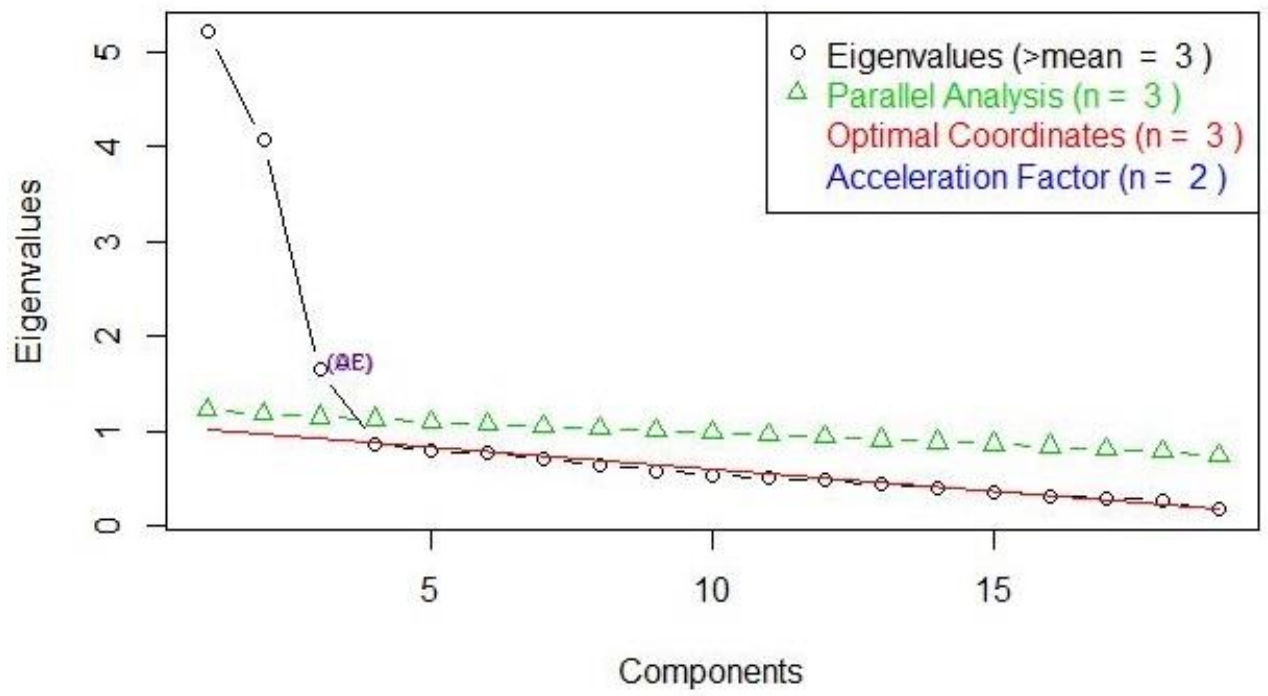

Figure 10. Scree test plot of gender role congruity variables.

The optimal number of factors suggested by the results of the scree plot was three, as opposed the two commonly utilized by Diekman et al. $(2010,2017)$. The factor analysis results using a three-factor solution revealed an additional agentic dimension. The original agentic factor was therefore split into two factors as represented by what can be described as "extrinsic agentic" factors, and "intrinsic agentic" factors. For the remainder of this study, reference to Diekman's gender role congruity will consider the three factors of communal, extrinsic agentic, and intrinsic agentic roles rather than the more simplified two factors theorized in the first three chapters based on prior work. Furthermore, five items were also removed that did not load above 0.4 even in the three factor solution (Osborne, Costello, \& Kellow, 2008; Tabachnick \& Fidell, 2007). These items were: two communal dimensions of "spirituality" (Q19I) and "intimacy" (Q19J), and three agentic dimensions of "achievement" (Q19M), "mastery" (Q19S), and "focus 
on the self' (Q190). It is interesting that these items were removed because these are values that often show large gender differences (Jones et al., 2000). It may be that these items are not agentic for women the way that they are for men, or that these items are not important for women causing them to load differently in this data set comprised solely of females. The final factor analysis results for the remaining gender role congruity items are presented in Table 3:

Table 3

Gender Role Congruity Construct Validity

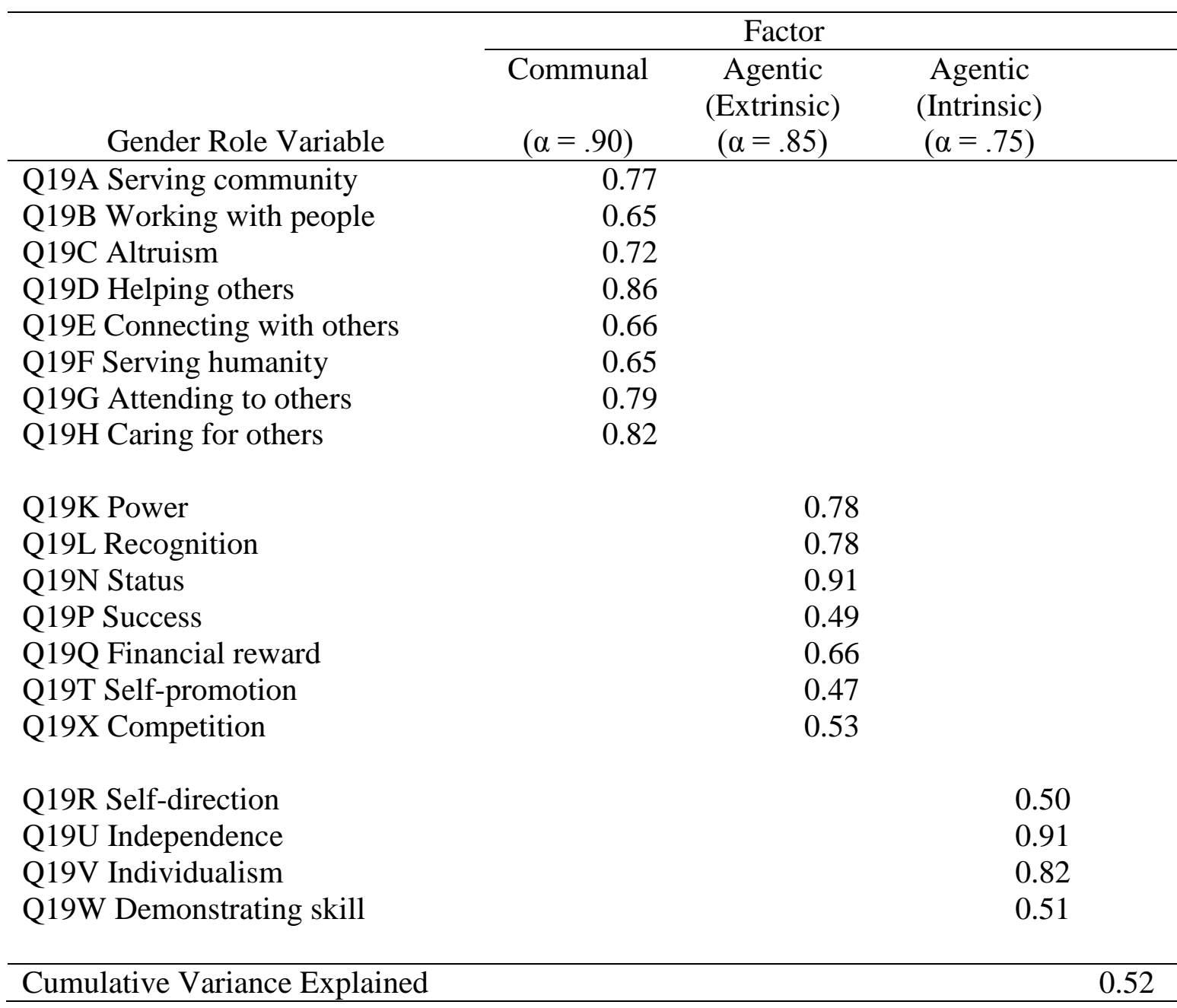


The results support the construct validity of the measures because the remaining items align appropriately to the newly theorized dimensions of communal, extrinsic agentic, and intrinsic agentic factors and the overall effect size over 0.5 is large (Tabachnick \& Fidell, 2007). Furthermore, the communal and agentic items theorized originally by Diekman and colleagues still loaded on separate factors. In terms of reliability, Cronbach's alpha was above the cutoff threshold of 0.7 for all three constructs. Specifically, for communal it was 0.90 , for extrinsic agentic it was 0.85 , and for intrinsic agentic it was 0.75 .

According to the list in question 19 of the CUWiP survey, communal gender roles can be defined with the following variables: serving the community, working with people, altruism, helping others, connecting with others, serving humanity, attending to others, caring for others. Extrinsic agentic can be described as: power, recognition, status, success, financial reward, self-promotion, and competition. Intrinsic agentic can be described as: self-direction, independence, individualism, and demonstrating skill. Descriptive statistics for the communal, extrinsic agentic, and intrinsic agentic gender roles are shown in Table 4:

Table 4

Gender Role Congruity Descriptive Statistics

\begin{tabular}{lcccc}
\hline Gender Role Variable $^{\mathrm{a}}$ & $M$ & $S D$ & Min & Max \\
\hline Communal & 3.11 & 0.75 & 0 & 4 \\
Agentic (Extrinsic) & 2.60 & 0.72 & 0 & 4 \\
Agentic (Intrinsic) & 3.30 & 0.63 & 1 & 4 \\
\hline${ }^{\mathrm{a}} n=900$ & & & &
\end{tabular}




\section{Identity and "Ideal" Science Student/“'Ideal" Scientist}

CUWiP participants answered the pre-conference survey questions 5 which

addressed the identity and the "ideal" physics student dimension of recognition. These

items are summarized in Figure 11:

If applicable, to what extent do you believe the following people...

\begin{tabular}{|c|c|c|c|}
\hline & Not at all & $\begin{array}{lllll}0 & 1 & 2 & 3 & 4\end{array}$ & Very much so \\
\hline ....see & u as an exemplary physics student? & & \\
\hline Q5A & Yourself & $\mathrm{O} O \mathrm{OOO}$ & \\
\hline Q5B* & Your HS physics teacher(s) & $\mathrm{O} O \mathrm{OOO}$ & \\
\hline Q5C & Other physics undergraduates & $\mathrm{OOOOO}$ & \\
\hline Q5D & Your physics professors/faculty & $\mathrm{OOOOO}$ & \\
\hline $\begin{array}{l}\text {...see } \\
\text { exemp }\end{array}$ & $\begin{array}{l}\text { lother physics student you know as an } \\
\text { ary physics student? }\end{array}$ & & \\
\hline Q5E & Yourself & $\mathrm{OOOOO}$ & \\
\hline $\mathrm{Q}_{5} \mathrm{~F}^{\wedge}$ & Your HS physics teacher(s) & $\mathrm{OOOOO}$ & \\
\hline Q5G & Other physics undergraduates & $\mathrm{O} O \mathrm{OOO}$ & \\
\hline Q5H & Your physics professors/faculty & $\mathrm{O} O \mathrm{OOO}$ & \\
\hline
\end{tabular}

Figure 11. CUWiP pre-conference survey question 5: Identity and ideal.

*indicates item was removed after loading below the 0.4 cutoff during the factor analysis $\wedge$ indicates item was removed in order to compare variables consistently, and corresponding variable was removed due to loading below the 0.4 cutoff

CUWiP participants answered the pre-conference survey questions 6 which addressed the identity and the "ideal" science student dimensions of interest, competence, and performance, featured in Figure 12: 


\begin{tabular}{|c|c|c|}
\hline \multicolumn{2}{|c|}{$\begin{array}{l}\text { To what extent do you believe the } \\
\text { following people... }\end{array}$} & $\begin{array}{lllllll}\text { Not at all } & 0 & 1 & 2 & 3 & 4 & \text { Very much so }\end{array}$ \\
\hline \multicolumn{3}{|c|}{$\begin{array}{l}\text {...have a personal interest in physics course } \\
\text { topics/concepts? }\end{array}$} \\
\hline Q6A & Yourself & $\mathrm{OOOOO}$ \\
\hline Q6B & Most exemplary physics student you know & $\mathrm{O} O \mathrm{OOO}$ \\
\hline \multicolumn{3}{|c|}{$\begin{array}{l}\text {...have a personal interest in physics topics outside } \\
\text { of courses? }\end{array}$} \\
\hline Q6C & Yourself & $\mathrm{OOOOO}$ \\
\hline Q6D & Most exemplary physics student you know & $\mathrm{O} O \mathrm{OOO}$ \\
\hline \multicolumn{3}{|c|}{...have interest in conducting physics research? } \\
\hline Q6E & Yourself & $\mathrm{OOOOO}$ \\
\hline Q6F & Most exemplary physics student you know & $\mathrm{O} O \mathrm{O} O \mathrm{O}$ \\
\hline \multicolumn{3}{|c|}{...have interest in things other than physics? } \\
\hline 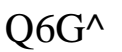 & Yourself & $\mathrm{OOOOO}$ \\
\hline Q6H* & Most exemplary physics student you know & $\mathrm{O} O \mathrm{O} O \mathrm{O}$ \\
\hline \multicolumn{3}{|c|}{...understand physics topics/concepts well? } \\
\hline Q6I & Yourself & $\mathrm{OOOOO}$ \\
\hline Q6J & Most exemplary physics student you know & $\mathrm{OOOOO}$ \\
\hline \multicolumn{3}{|c|}{...understand physics research/experiments well? } \\
\hline Q6K & Yourself & $\mathrm{O} O \mathrm{OOO}$ \\
\hline Q6L & Most exemplary physics student you know & $\mathrm{OOOOO}$ \\
\hline \multicolumn{3}{|c|}{$\begin{array}{l}\text {...have the ability to do physics } \\
\text { research/experiments well? }\end{array}$} \\
\hline Q6M & Yourself & $\mathrm{OOOOO}$ \\
\hline Q6N & Most exemplary physics student you know & $\mathrm{O} O \mathrm{O} O \mathrm{O}$ \\
\hline \multicolumn{3}{|c|}{$\begin{array}{l}\text {...have the ability to do well in difficult physics } \\
\text { courses? }\end{array}$} \\
\hline 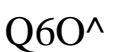 & Yourself & $\mathrm{OOOOO}$ \\
\hline Q6P* & Most exemplary physics student you know & $\mathrm{OOOOO}$ \\
\hline \multicolumn{3}{|c|}{$\begin{array}{l}\text {...have the ability to explain/communicate physics } \\
\text { to others well? }\end{array}$} \\
\hline Q6Q* & Yourself & $\mathrm{O} O \mathrm{OOO}$ \\
\hline Q6R* & Most exemplary physics student you know & $\mathrm{OOOOO}$ \\
\hline
\end{tabular}

Figure 12. CUWiP pre-conference survey question 6: Identity and ideal.

*indicates item was removed after lower loading compared to other items during the factor analysis

$\wedge$ indicates item was removed in order to compare variables consistently, and corresponding variable was removed due to loading at or below the 0.4 cutoff

As shown in Table 5, the following items were pulled out of the factor analysis for science identity because they were loading too low, or to include variables 
consistently across identity and "ideal" physics student ( ${ }^{\wedge}$ was used to indicate variables whose factor analysis loaded at or above the 0.4 cutoff (Tabachnick \& Fidell, 2007), but following the factor analysis and promax rotation analysis, the corresponding variable for the "ideal" science student was below, therefore both were removed): High School physics teacher seeing student as exemplary (Q5B), interest in things other than physics $(\mathrm{Q} 6 \mathrm{G})$, ability to do well in difficult physics courses $\left(\mathrm{Q6O}^{\wedge}\right)$, and ability to explain/communicate physics to others well (Q6Q). The remaining items all loaded as expected onto the theorized sub-constructs of recognition, interest, and performance/competence beliefs. Table 10 displays the final factor analysis of participants' responses to identity-related prompts in questions 5 and 6 as follows:

Table 5

Factor Analysis of Physics Identity for Self

\begin{tabular}{lccc}
\hline \multicolumn{1}{c}{$\begin{array}{c}\text { Physics Identity } \\
\text { Variable }\end{array}$} & $\begin{array}{c}\text { Recognition } \\
(\alpha=0.80)\end{array}$ & $\begin{array}{c}\text { Interest } \\
(\alpha=0.82)\end{array}$ & $\begin{array}{c}\text { Competence } \\
(\alpha=0.79)\end{array}$ \\
\hline Q5A Recognition & 0.69 & & \\
Q5C Recognition & 0.79 & & \\
Q5D Recognition & 0.94 & 0.82 & \\
Q6A Interest & & 0.80 & 0.42 \\
Q6C Interest & & 0.59 & $1.10^{*}$ \\
Q6E Interest & & & 0.65 \\
Q6I Competence & & & \\
Q6K Competence & & & \\
Q6M Performance & & &
\end{tabular}

\footnotetext{
Cumulative Variance 0.63

*Note: Factor loadings greater than 1 are possible with promax rotations

This supports the construct validity of the measures. To create an overall physics identity measure, each sub-construct was first created by averaging the items loading in the
} 
aligned factor and then all three sub-constructs were averaged for the overall physics identity measure.

In terms of reliability, Cronbach's alpha was above the cutoff threshold of 0.7 for the items in the overall physics identity measure. Specifically, the median alpha was 0.82 indicating a high degree of internal consistency. Thus the reliability of the measures for physics identity are supported by the data.

As shown in Table 5, the following items were pulled out of the factor analysis for the "ideal" science student because they were loading too low, or in order to compare variables consistently $(\wedge$ was used to indicate variables whose factor analysis loaded at or above the 0.4 cutoff, but the corresponding variable for identity was below, therefore both were removed): High School physics teacher seeing another physics student as exemplary $\left(\mathrm{Q} 5 \mathrm{~F}^{\wedge}\right)$, interest in things other than physics for the most exemplary physics student they know (Q6H), ability to do well in difficult physics courses for the most exemplary physics student they know (Q6P^), and ability to explain/communicate physics to others well for the most exemplary physics student they know (Q6R). The remaining items all loaded as expected onto the theorized sub-constructs of recognition, interest, and performance/competence beliefs, which supports the construct validity of the items. Table 6 displays the final factor analysis results for the students' assessment of the "ideal" student as follows: 
Table 6

Factor Analysis of "Ideal” Physics Student

\begin{tabular}{lccc}
\hline \multirow{2}{*}{ “Ideal” Variable } & $\begin{array}{c}\text { Recognition } \\
(\alpha=0.78)\end{array}$ & $\begin{array}{c}\text { Interest } \\
(\alpha=0.79)\end{array}$ & $\begin{array}{c}\text { Competence } \\
(\alpha=0.77)\end{array}$ \\
\cline { 2 - 4 } Q5E Recognition & 0.69 & & \\
Q5G Recognition & 0.89 & & \\
Q5H Recognition & 0.84 & 0.72 & 0.63 \\
Q6B Interest & & 0.70 & $1.03 *$ \\
Q6D Interest & & 0.54 & 0.59 \\
Q6F Interest & & & \\
Q6J Competence & & & 0.57 \\
Q6L Competence & & & \\
Q6N Performance & & & \\
\multicolumn{2}{c}{ Cumulative } \\
$\quad$ Variance
\end{tabular}

*Note: Factor loadings greater than 1 are possible with promax rotations

The "ideal" physics student measure was created by combining the items in the same way they were combined for the self-physics identity measure. In terms of reliability, Cronbach's alpha for all the "ideal" physics student items was above the cutoff threshold of 0.7. Specifically, it was 0.80 across all factors; thus, the reliability of the measures for the "ideal" physics student is also supported by the data.

Table 7 features descriptive statistics for identity and the "ideal" physics student:

Table 7

Physics Identity and the "Ideal" Physics Student Descriptive Statistics

\begin{tabular}{lcccc}
\hline Variable & $M$ & $S D$ & Min & Max \\
\hline Identity & 3.05 & 0.54 & 0.78 & 4 \\
"Ideal" Physics Student & 3.53 & 0.41 & 1.78 & 4 \\
\hline
\end{tabular}

${ }^{\mathrm{a}} n=900$ 


\section{Findings}

To conduct the statistical tests for this study, the raw data from the CUWiP preconference survey were uploaded in R. To reference the code written to analyze this data, please refer to Appendix I. A series of linear regression analyses were run to determine the significant relationships between the four concepts making up the framework for this study. Multicollinearity using variance inflation factors were conducted on all regressions with more than one independent variable, and were found to be below 2 in all cases (ranging from 1.04-1.28) (Tabachnick \& Fidell, 2007). The remainder of this section contains the results of the statistical analysis for each of the three research questions addressed by this phase of the study.

\section{Research Question 1}

The first of the research questions governing this study was: Is the way undergraduate women in physics see the "ideal" physics student related to their physics identity? The results of this linear regression showed that a significant correlation at the $p<0.001$ level exists between physics identity and the "ideal" science student. Table 8 contains the regression coefficients for the linear regression:

Table 8

Perceptions of the "Ideal" Science Student as Predictor of Physics Identity

\begin{tabular}{lcccc}
\hline \multicolumn{1}{c}{ Predictor } & $\beta^{\wedge}$ & $S E$ & $p$ & $R^{2}$ \\
\hline $\begin{array}{l}\text { Perceptions of the "Ideal" } \\
\text { Physics Student }\end{array}$ & 0.20 & 0.04 & $2.78 \mathrm{e}-09 * * *$ & 0.04 \\
\hline
\end{tabular}

$* * *$ Correlation is significant at the level of 0.001

$\wedge$ Note: For a single linear regression $\beta$ is the same as the Pearson Correlation $r$

The correlation between these constructs is significant; however, the effect size is small. (Tabachnick \& Fidell, 2007). 


\section{Research Question 2}

The second research question was: How does gender role congruity relate to identity? To appropriately look at these relationships, first a correlation test was run to determine the relationships between identity as the dependent variable and the following as independent variables: communal goals, extrinsic agentic goals, and intrinsic agentic goals. Table 9 contains the regression coefficients for the linear regression run on these variables separately:

Table 9

Gender Goals as Individual Predictors of Physics Identity

\begin{tabular}{lcccc}
\hline \multicolumn{1}{c}{ Predictor } & $\beta^{\wedge}$ & $S E$ & $p$ & $R^{2}$ \\
\hline Communal Goals & 0.14 & 0.02 & $2.11 \mathrm{e}-05^{* * *}$ & 0.02 \\
Extrinsic Agentic Goals & 0.16 & 0.03 & $1.52 \mathrm{e}-06^{* * *}$ & 0.03 \\
Intrinsic Agentic Goals & 0.28 & 0.03 & $<2 \mathrm{e}-16^{* * *}$ & 0.08 \\
\hline
\end{tabular}

*** Correlation is significant at the level of 0.001

${ }^{\wedge}$ Note: For a single linear regression $\beta$ is the same as the Pearson Correlation $r$

In this case, a significant correlation exists at the $p<0.001$ level between each of the three gender goals and identity when these relationships are examined individually (i.e., identity-communal, identity-extrinsic agentic, identity-intrinsic agentic), with the largest effect for intrinsic agentic goals. Although each of these effect sizes is relatively small, they are noteworthy because there are many factors that contribute to identity (Tabachnick \& Fidell, 2007).

Additionally, a multiple linear regression was run to look at the relationship between all three gender goals combined and identity. In this case the results showed that a significant correlation at the $p<0.001$ level exists between the intrinsic agentic goals and identity for the undergraduate female physics majors in this study. Additionally, a smaller 
but significant correlation at the $p<0.01$ level exists between the communal goals and identity for these same participants. No significant correlation exists between the extrinsic agentic goals and a physics identity for women majoring in physics or engineering in college. This correlation is depicted in Table 10:

Table 10

Linear Regression for Gender Goals and Physics Identity

\begin{tabular}{lcrrr}
\hline \multicolumn{1}{c}{ Variable } & $\beta$ & \multicolumn{1}{c}{$S E$} & $p$ & $R^{2}$ \\
\hline Communal Goals & 0.11 & 0.02 & $0.0011^{* * *}$ & \\
Extrinsic Agentic Goals & 0.05 & 0.03 & 0.13 & \\
Intrinsic Agentic Goals & 0.25 & 0.03 & $<2.32 \mathrm{e}-11^{* * *}$ & 0.09 \\
\hline
\end{tabular}

*** Correlation is significant at the level of 0.001

** Correlation is significant at the level of 0.01

Examining these relationships between gender roles and identity individually and in combination allows us to see the ways in which each of these gender roles have a significant relationship with identity for undergraduate women majoring in physics, but highlights the particularly critical nature of intrinsic agency for these women, and the relatively insignificant relationship between extrinsic agency and identity when tested in combination with the other two dimensions of gender roles. Similar to the earlier discussion about the correlation tests between physics identity and the gender goal dimensions individually, although an overall effect size of 0.09 is considered small, seeing gender as nearly $10 \%$ of the contributing factors of a physics identity for women is helpful to understanding identity development in this context (Tabachnick \& Fidell, 2007). 


\section{Research Question 3}

The final research question in focus for this phase of the study was: What factors (i.e., physics identity, "ideal" scientist, gender role congruity) relate to plans to persist? Persistence served as the dependent variable measured in three self-reported levels (career intentions, graduate school intentions, and bachelor's degree attainment intentions), while identity, the "ideal" scientist, and gender role congruity served as the independent variables. To accomplish this, data reported on the CUWiP pre-conference survey was pulled from questions 1, 2, and 3, as indicated by Figure 13:

\begin{tabular}{|llllllll|}
\hline $\begin{array}{l}\text { 1. To what extent would you consider } \\
\text { pursuing the following careers with a }\end{array}$ & Not at all & 0 & 1 & 2 & 3 & 4 & Very much so \\
background in physics: & & & & & \\
\hline Q1A & Professor & O O O O O & \\
Q1B Industry Scientist & O O O O O & \\
Q1C & Research/lab scientist & O O O O O & \\
Q1D & Engineer & O O O O O O \\
\hline
\end{tabular}

2. Do you plan to attend graduate school in physics?

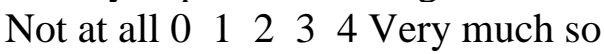

3. Do you intend to complete a bachelors degree in physics?

Not at all $0 \begin{array}{lllll}1 & 2 & 3 & 4 & \text { Very much so }\end{array}$

Figure 13. CUWiP pre-conference survey questions 1, 2, and 3: Persistence.

The options professor, industry scientist, research/lab scientist, and engineer were combined to create one "career plans" variable based on the maximum value of students' responses to these career plans. Table 11 features descriptive statistics for persistence broken down by the three questions above in the categories of bachelors degree completion, plans to attend graduate school in physics, and career plans: 
Table 11

Persistence Self-reported as Academic and Career Plans Descriptive Statistics

\begin{tabular}{lccccc}
\hline Persistence Category & $n$ & $M$ & $S D$ & Min & Max \\
\hline Bachelor's Degree & 898 & 3.79 & 0.72 & 0 & 4 \\
Graduate School & 897 & 3.15 & 1.13 & 0 & 4 \\
Career Plans & 887 & 3.72 & 0.64 & 0 & 4 \\
\hline
\end{tabular}

A series of linear regressions were run to address the third research question. A significant correlation between identity and self-reported plans to persist to bachelor's degree graduation in physics exists at the $p<0.001$ level. Additionally, communal goals have a significant negative correlation with bachelor's degree persistence at the $p<0.05$ level. This is an important finding because it confirms the premise of this research question. Intrinsic agency, extrinsic agentic goals, and perceptions of the "ideal" scientist have no significant correlation with self-reported plans to persist to graduation with a bachelor's degree in physics. These results are displayed in Table 12:

Table 12

Linear Regression for Physics Identity, the "Ideal" Physics Student, and Gender Goals as Predictors of Self-Reported Bachelor's Degree Persistence

\begin{tabular}{lcccc}
\hline \multicolumn{1}{c}{ Predictor } & $\beta$ & $S E$ & $p$ & $R^{2}$ \\
\hline Physics Identity & 0.21 & 0.05 & $9.69 \mathrm{e}-09 * * *$ & \\
"Ideal" Physics Student & -0.02 & 0.06 & 0.63 & \\
Communal Goals & -0.09 & 0.03 & $0.012^{*}$ & \\
Extrinsic Agentic Goals & -0.07 & 0.04 & 0.07 & \\
Intrinsic Agentic Goals & -0.04 & 0.05 & 0.27 & \\
& & & & 0.04 \\
\hline
\end{tabular}

$* * *$ Correlation is significant at the level of 0.001

* Correlation is significant at the level of 0.05

Examining the same set of constructs for their relationships with self-reported plans to persist to a graduate degree in physics resulted in a significant correlation 
between identity and graduate school plans at the $p<0.001$ level, and a significant correlation between intrinsic agency and grad school plans at the $p<0.05$ level. No significant relationships were identified between communal goals, extrinsic agency, nor perceptions of the "ideal" science student and plans to persist to graduate school in physics. Table 13 displays these results:

Table 13

Linear Regression for Physics Identity, the "Ideal" Physics Student, and Gender Goals as Predictors of Self-Reported Graduate Degree Persistence

\begin{tabular}{lcccc}
\hline \multicolumn{1}{c}{ Predictor } & $\beta$ & $S E$ & $p$ & $R^{2}$ \\
\hline Physics Identity & 0.35 & 0.07 & $<2 \mathrm{e}-16 * * *$ & \\
"Ideal" Physics Student & -0.03 & 0.09 & 0.33 & \\
Communal Goals & -0.04 & 0.05 & 0.28 & \\
Extrinsic Agentic Goals & -0.05 & 0.06 & 0.20 & \\
Intrinsic Agentic Goals & 0.09 & 0.07 & $0.024 *$ & \\
& & & & 0.13
\end{tabular}

$* * *$ Correlation is significant at the level of 0.001

* Correlation is significant at the level of 0.05

Finally, an examination of the same set of constructs for their relationships with self-reported plans to persist to a career in a physics-related field of work resulted in a significant correlation between identity and all self-reported career plans at the $p<0.001$ level. A significant correlation exists between intrinsic agency and career plans at the $p<0.05$ level. No significant relationships were identified between communal goals, extrinsic agency, and perceptions of the "ideal" scientist and the physics-related careers available in CUWiP question 1 on career plans. These results are displayed in Table 14: 
Table 14

Linear Regression for Physics Identity, the "Ideal" Physics Student, and Gender Goals as Predictors of Self-Reported Career Plans as Persistence

\begin{tabular}{lcccc}
\hline \multicolumn{1}{c}{ Predictor } & $\beta$ & $S E$ & $p$ & $R^{2}$ \\
\hline Physics Identity & 0.29 & 0.04 & $8.18 \mathrm{e}-16 * * *$ & \\
"Ideal” Physics Student & -0.01 & 0.05 & 0.79 & \\
Communal Goals & -0.02 & 0.03 & 0.47 & \\
Extrinsic Agentic Goals & 0.03 & 0.03 & 0.37 & \\
Intrinsic Agentic Goals & 0.09 & 0.04 & $0.0232 *$ & \\
& & & & 0.11 \\
\hline
\end{tabular}

$* * *$ Correlation is significant at the level of 0.001

* Correlation is significant at the level of 0.05

This examination of each of the pieces of the conceptual framework and their relationships to the self-reported plans to persist therefore indicates that identity always has a significant correlation for female undergraduate students majoring in physics. Additionally, the regression beta weights ranged from -0.08 to 0.74 , supporting the empirical and theoretical relevance of understanding the impact of gender role congruity, science identity, and perceptions of the "ideal" science student on intentions to persist for undergraduate female physics majors.

Although gender roles and goals do also have a relationship with the students' plans to persist, the significance of these relationships is less than that of identity. Overall, the effect sizes of these factors on long-term goals, while small at 0.13 and 0.11 , are still important to the overall findings of this portion of the study (Tabachnick \& Fidell, 2007). In no instances under examination for this study did the students' perceptions of the "ideal" science student indicate a significant relationship with their self-reported plans to persist. 


\section{Summary}

As stated earlier, this study examined data reported to APS by 900 undergraduate female students majoring in physical sciences in colleges and universities across the U.S. to better understand the relationships between physics identity, perceptions of the "ideal" scientist, gender role congruity, and plans to persist, as self-reported in the CUWiP preconference survey. A total of $94.4 \%$ of the sample self-identified as female, all of whose answers were used for the subsequent statistical analyses described throughout this chapter. After running a factor analysis on each of the items under investigation for this study, the items were determined suitable for a series of linear regressions. A notable finding from the factor analysis portion of this phase was the discovery that the previously examined binary gender congruity roles should in fact be examined in the ternary through the splitting of agentic roles into two separate dimensions of gender role congruity, that of extrinsic agency and intrinsic agency.

This chapter also provided the results of the series of linear regression analyses that were conducted to answer the three research questions under examination for this quantitative phase of the study. It was noted that identity plays a significant role for students planning to persist at any level in physical science fields. The results also indicated that intrinsic agentic gender roles and goals are highly predictive of participants' self-reported long-term persistence plans $(p<0.001)$, such as graduate school and career plans beyond their bachelor's degree completion. Furthermore, communal roles were negatively correlated with students' short-term persistence defined by physics bachelor's degree completion, which raises questions about whether or not communal women feel like they fit in, or that physics will fulfill their future needs, and thus whether 
or not they are more likely to consider quitting. Extrinsic agency was correlated with neither identity nor persistence. Considering relationships (or lack thereof) of the three concepts with the students' plans to persist in the field could significantly aid higher education faculty and administrators in prioritizing approaches and resources to increasing persistence of gender diversity in male-dominated STEM fields such as physical sciences. Additionally, considering the significant correlations between female undergraduates' physics identity and communal, extrinsic agentic, and intrinsic agentic gender roles and goals could provide faculty and administrators with additional tools to determine effective interventions aimed at closing the gender gap in physics.

This chapter covered all aspects of the quantitative strand of this study, including the population demographics for the 2016 CUWiP data, construct validity and reliability, and the findings for the series of regressions. A more in depth interpretation of these results and how they can be used in the future by higher education faculty, administration, and researchers will be discussed in greater detail in Chapter VI. The next chapter, Chapter V, will outline the details of the qualitative strand of the study. 


\section{CHAPTER V}

\section{QUALITATIVE FINDINGS}

As discussed in previous chapters, the purpose of this study was to examine the relationships between the constructs of gender role congruity, physics identity, the "ideal" scientist, and plans to persist in a physical science major for undergraduate female students via an explanatory sequential mixed methods research design. This chapter presents the qualitative results of the study and is organized into three major sections followed by a summary: participants, a presentation of the findings which has been organized into five phenomenographic categories of experience, and a discussion of the outcome space as defined by these five categories. For the quantitative results of this study, please see Chapter 4; and, for a complete discussion of the research questions (first presented in Chapter 3) as well as the interpretation of the mixed methods findings, please see Chapter 6.

\section{Participants}

As discussed in the third chapter, to collect a diverse qualitative sample, a sincere effort to consider multiple aspects of participant diversity in this phase of the study was made. After contacting a total of 253 participants from the quantitative sample who met the criteria for the qualitative strand (e.g. female gender identity, year in school, minimum number of physics courses taken, etc.), a total of 60 responses were received (a response rate of $23.7 \%$ ). With a higher number of responses than the 15-20 goal for this strand of the study, I was able to be somewhat selective when it came to racial and ethnic diversity, geographic diversity, institution type diversity, and diversity in their responses to the CUWiP survey questions related to this study. After participants responded to my 
initial email invitation, they were sent a follow up email either requesting to schedule an interview, or a notification that due to a high volume of responses an interview may be scheduled at a later date if needed. A total of 18 interviews averaging 60 minutes in length were conducted, and an additional four participants were scheduled for interviews but did not complete the interview or follow up further. Seventeen of the 18 interviews were conducted by phone, and one interview was conducted through Skype. All 18 interviews were transcribed and analyzed. While I was prepared to conduct more interviews and the sample was available, the broad patterns observed in the constructs were saturated with 18 interviews indicating that these interviews were sufficient to represent the phenomenographic categories. After it was determined that sufficient data was collected from the 18 interviews, the 18 participants interviewed were contacted two additional times each, once by email with a copy of the transcript for their review and feedback, and once by United States Postal Service mail to provide them with a thank you note and the Starbucks gift cards promised in the original email invitation. For additional details regarding the qualitative participant recruitment and selection processes, please see Chapter 3 and the appropriate appendices.

Of the 18 students who participated in the interviews, all students were female undergraduate students majoring in physics, astrophysics, or engineering physics with 11 participants also carrying a double major or minor in fields such as engineering, astronomy, mathematics, or another academic area not under investigation for this study. All students attended a university in the U.S., with one student attending a university outside of the contiguous U.S. The interviews were conducted throughout the summer of 2016, therefore four participants in this phase of the study had recently graduated with 
their bachelor's degree, with varying plans ranging from graduate school in their field, graduate school in a STEM field other than physics or engineering, job searching, and life planning. The remainder of the qualitative participants were conducting research through the NSF funded Research Experiences for Undergraduates (REU) program in the U.S. and abroad, or had other summer engagements such as internships and jobs. Figure 14 provides a visual representation of the approximate geographic locations of the universities attended by participants in the qualitative strand of this study:

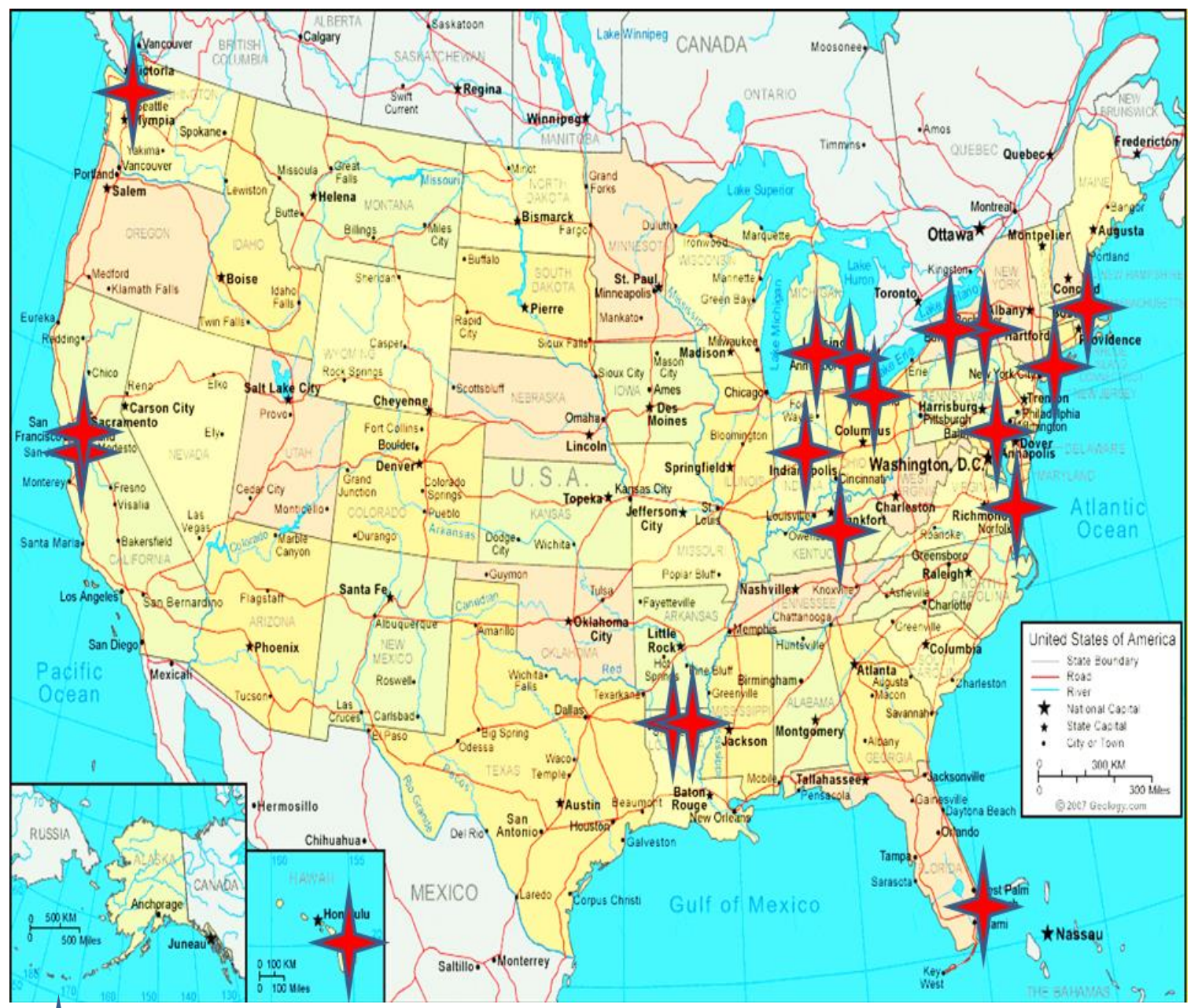

The approximate university location attended by participants.

Figure 14. Map of approximate geographic location of qualitative participants' undergraduate university. 
In addition to the geographic location of the university attended by the participants, I knew a series of other identifying pieces of information about each participant as I conducted the interviews. For additional details about the qualitative sample, please refer to Chapter 3 and the appropriate appendices.

The 18 participants featured throughout the findings of this chapter were assigned pseudonyms and reasonable attempts have been made to mask identifying information found within direct quotes using brackets to replace proper nouns with generic references in order to protect the identity of the participants. Ellipses have been used to remove redundant or excess words and phrases. An overview of the participants' pseudonyms, race/ethnicity, major(s), and other information that will be used in this chapter is presented in the next section.

\section{Findings}

Utilizing a phenomenographic approach to analyze the qualitative data related to gendered roles and goals, physics identity, and the "ideal" science student in physics, categories describing the qualitatively different ways these participants experience these phenomena began to emerge (Marton, 1981 \& 1986; Svensson, 1997). In later works on the phenomenographic approach to qualitative research, Marton and Booth stated:

The world is not constructed by the learner, nor is it imposed upon her; it is constituted as an internal relation between them. There is only one world, but it is a world that we experience [...] we are all different, and we do experience the world differently because our experience is always partial. (1997, p.13)

Beyond understanding this deeply constructivist paradigm bolstering the epistemological foundation of the method, Akerlind (2012) has further clarified the "variation of phenomenographic practice" (p. 115). This includes guiding procedures meant to 
delineate what constitutes the various phases of the analytic process and structuring the outcome space.

\section{Outcome Space Structure}

As discussed in Chapter 3, following five rigorous, multi-layered coding and analysis phases, a structured set of outcomes emerged from the data defining the outcome space (Akerlind, 2012). Akerlind (2012) suggests a series of collaborative steps to confirm that the set of outcomes represents, "the full range of possible ways of experiencing the phenomenon in question, at this particular point in time, for the population represented by the sample group collectively" (p. 116). Diekman et al. (2017) contextualize this further in that, "individuals also continually create their environment by entering, engaging in, and exiting specific roles" (p.142). Therefore, this outcome space was designed to categorically, holistically, and succinctly present the gamut of qualitatively different ways different participants experience their relationships with various aspects of the conceptual framework at the time of the interviews. This was achieved by grouping coded segments of participants' common physics identity dimensions such as interest, as well as competency and performance beliefs, and shared reflections about gender and the "ideal" across their experiences. These experiences with the conceptual framework were then grouped by relationship dimensions shared among participants, such as high competency beliefs or low competency beliefs. The depths of the similarities and differences between these shared relationships were explored, then organized by participants, forming five groups of participants whose relationships with the conceptual framework are defined as categories of experience. At each stage of grouping concepts, experiences, relationships, and finally participants, ongoing 
discussions and review of the data were held weekly with the principle investigator. Akerlind recommends this process take on a cyclical format by reviewing the data in full and framing a potential outcome space followed by collaborative discussions to clarify the outcome sets and subsets. This process is then conducted continuously until an approved set of outcomes endorsed by all researchers delineates a "more accurate" outcome space (Akerlind, 2012).

Even with this thorough, iterative analytic process, it must be acknowledged that this outcome space is complete but not absolutely accurate because, "any outcome space is inevitably partial, with respect to the hypothetically complete range of ways of experiencing a phenomenon" (p. 121). Therefore, the categories of experience presented below are defined as more or less, a "complete outcome space" based on the qualitative data, not generalizable as an absolute outcome space (Akerlind, 2012). Additionally, participants within each category of experience presented below may share some commonalities with participants in other categories; however, placement in a specific category of experience was based on the majority of their characteristics and reflections and the, "underlying meaning of virtually the whole transcript" rather than whether an individual quote or experience fit within a category of experience (Akerlind, 2012, p. 120).

For these reasons, the findings of the qualitative portion of the study, otherwise referred to throughout this chapter as the outcome space, are organized and displayed in the following sections through categories of experience entitled: The Assured, The Solitary, The Communal, The Reflective, and The Ambassadors. The nuances of the overall outcome space, including how borderline cases were decided, will be discussed 
immediately after the detailed delineation of these categories of experience. It is important to note here that the order in which the categories of experience are presented is hierarchically structured in terms of increasing complexity and inclusivity in the relationships participants experience with the conceptual framework, as recommended by Marton and Booth (1997). In other words, the fact that The Assured is presented first, for example, should not signify any rank or privilege to participants who experience this type of relationship with the conceptual framework, rather that this category is marked by a more straightforward manner by which participants experience their relationship with the conceptual framework. A brief overview of the 18 qualitative participants including identifying information that will be utilized throughout the remainder of this chapter is featured in Table 15 below (listed in the same order as the CUWiP survey scores list in Chapter 3): 
Table 15

Qualitative Participant Demographics

\begin{tabular}{|c|c|c|c|c|}
\hline Pseudonym & $\begin{array}{l}\text { Race/ } \\
\text { Ethnicity }\end{array}$ & $\begin{array}{l}\text { Major(s)(M)/ } \\
\text { Minor(s)(m) }\end{array}$ & $\begin{array}{l}\text { Category of } \\
\text { Experience }\end{array}$ & Institution Type/Description \\
\hline Hilary & White, NAAN** & $\begin{array}{l}\text { Physics }(\mathrm{M}) \\
\text { Astronomy }(\mathrm{m})\end{array}$ & Assured & Mid-sized*, public university \\
\hline Madelyn & White, Hispanic & Physics(M) & Assured & $\begin{array}{l}\text { Large*, public, research, Hispanic } \\
\text { Serving Institution (HSI) }\end{array}$ \\
\hline Samantha & White, Hispanic & $\begin{array}{l}\text { Physics(M) Classical } \\
\text { Civilizations(M) }\end{array}$ & Assured & $\begin{array}{l}\text { Small*, private, liberal arts, women's } \\
\text { college }\end{array}$ \\
\hline Stella & White & $\begin{array}{l}\text { Astrophysics(M) } \\
\text { Advanced Math(M) }\end{array}$ & Assured & Large, public, research university \\
\hline Brooklyn & White, Hispanic & Physics(M) & Solitary & Small, public, state university \\
\hline Dakota & White & Physics(M) & Solitary & Small, public, liberal arts college \\
\hline Delores & White, Hispanic & Physics(M) & Solitary & Large, public university \\
\hline Gloria & White & $\begin{array}{l}\text { Physics(M), } \\
\text { Mathematics(M) }\end{array}$ & Solitary & Mid-sized, public, research university \\
\hline Matilda & White & $\begin{array}{l}\text { Physics }(\mathrm{M}), \\
\text { Chemistry }(\mathrm{m})\end{array}$ & Solitary & Small, private, liberal arts college \\
\hline Gabriella & White & Physics(M) & Communal & Small, private university \\
\hline Marion & White & Physics(M) & Communal & Large, public, research university \\
\hline Naomi & Black & $\begin{array}{l}\text { Physics(M), } \\
\text { Mathematics(M) }\end{array}$ & Communal & $\begin{array}{l}\text { Small, public, Historically Black } \\
\text { College or University (HBCU) }\end{array}$ \\
\hline Carly & White, Hispanic & $\begin{array}{l}\text { Physics(M), } \\
\text { Mathematics(m) }\end{array}$ & Reflective & $\begin{array}{l}\text { Small, private, religiously affiliated } \\
\text { university }\end{array}$ \\
\hline Dahlia & Asian & Physics(M) & Reflective & $\begin{array}{l}\text { Mid-sized, public/private research } \\
\text { university }\end{array}$ \\
\hline Vanessa & White & $\begin{array}{l}\text { Physics(M) } \\
\text { Electrical } \\
\text { Engineering(M) }\end{array}$ & Reflective & Mid-sized, public, university \\
\hline Yvette & White & $\begin{array}{l}\text { Engineering Physics(M) } \\
\text { Mathematics(M) }\end{array}$ & Reflective & $\begin{array}{l}\text { Mid-sized, private, religiously affiliated, } \\
\text { research university }\end{array}$ \\
\hline Bethany & $\begin{array}{l}\text { Asian, Hispanic, } \\
\text { NPHI** }\end{array}$ & $\begin{array}{l}\text { Physics(M), } \\
\text { Astrophysics(M) }\end{array}$ & Ambassador & Large, public, research university \\
\hline Stephanie & $\begin{array}{l}\text { Black, } \\
\text { Hispanic }\end{array}$ & $\begin{array}{l}\text { Physics(M) } \\
\text { Mechanical } \\
\text { Engineering(M) }\end{array}$ & Ambassador & Small, private university \\
\hline
\end{tabular}

\footnotetext{
*Small-fewer than 6,000 undergraduates; Mid-sized-6,001-15,000; Large-greater than 15,000

**Native American or Alaskan Native (NAAN), Native Hawaiian or Pacific Islander (NPHI)
} 


\section{The Assured}

Contextualizing the experiences of the assured within the conceptual framework of this study reveals congruent language depicting a well-developed physics identity based on high competency and performance beliefs, robust recognition examples, and longstanding and deep interest in their major. The assured also describe high levels of grit, ambition, and a work ethic to match; however, the ways the assured experience and attribute their success is as unique as each of the four participants identified for this group. Some attribute their success to hard work, or their environment, while some humbly attribute their success to their interests and their ability to "grow into the community." For all of the assured, their major choices seem obvious, as if the path had been set for them and they are following it with confidence.

\section{Identity Congruence}

The well-developed physics identity of the assured serves as a foundation for congruence with other aspects of the conceptual framework, such as gender and "ideal" assumptions. Each of the participants in this category of experience: Hilary, Madelyn, Samantha, and Stella shared multiple examples of the dimensions of their physics identity, marked by early interest, high competency and performance beliefs, and a variety of types and sources of recognition.

Early interest. As far back as she can remember, Samantha has always wanted to major in physics, making her decision to double major in physics and classical civilizations at a small, private, liberal arts, women's college in the northeast a logical next step; this combination of early interest and environment influences her experiences as a physics major. Madelyn shared, "I really liked math and science from the beginning. 
... I was like, 'I have fun doing this stuff. . .so why should I stop having fun doing something. . . that I enjoy?"” Stella thinks she deserves to study what excites her; she expressed deep interest in her studies: "Every time I look at the night sky, I just feel really happy that I'm learning about it and doing my own research on it." And despite never having taken a physics class prior to college, Hilary realized that she loved the Discovery Channel and had fond memories of learning about physics concepts from her father, so she took physics her first semester and now is confidently working towards her bachelor's degree in physics and astronomy.

High competence and performance beliefs. Both Hilary and Madelyn are willing to describe themselves as among the top in their classes. Hilary cites herself as the exemplar when asked to describe the most successful student in her class: "Okay, this is going to sound a little bit arrogant, but I am the good student in my physics classes." When asked if she considers herself at the top of the class, Madelyn replied, "Yeah, I'd say so. I do put a lot of work into it, so yeah, I'd say I'm among the top." Hilary also cites her hard work, commitment, and time management as the source of her high performance outcomes in the form of good grades. When probed to elaborate on characteristics possessed by the best students, Hilary responded:

Not necessarily characteristics, good students do tend to have advantages. For instance, I don't have a child to care about. I'm lucky that I have a scholarship and I don't need to work full time. That frees up a lot of time for studying that other people don't have. Another thing that really helps, I find, is that I have a good relationship with all of my professors. I go and talk to them after class. I research with them. I also do a lot of things outside of school for my career, because I believe that grades aren't really as important as research.

I've done two REUs now. I spent half of last year in [another country] doing research. I've been working with a professor - not even for 
independent study, just to do research - for the last three and a half years. Yeah, it really helps to know your professors well and get them to respect you. . .Yeah. That helps a lot.

Hilary's examples go beyond stereotypical assumptions about brilliance required for physics, or merely putting in more time and hard work than her peers by also capturing her ambition to explore and utilize resources around her, and her willingness to actively build a network from which she can learn, grow, and benefit; her well-developed physics identity coupled with her high levels of intrinsic and extrinsic agency come across as clearly articulated confidence in her words and her choices. Not every participant in this category of experience expressed this level of agency, but they all described high levels of competence belief, even if they attributed the outcomes to different sources such as environment and interest.

For example, Samantha knows that her environment is an important part of her science identity development, her success, and her decision to continue in her major:

I definitely think that going to an all-girls school helped me gain the major and feel like I can do it. . . not being in a male dominated environment and not being told that I don't understand a concept that everyone else understood and blaming that on me being a woman, I think that really helped me stay in physics. It helped me feel more confident that if I don't understand something, it's not because I'm a woman, it's because I just didn't understand it and I can learn it. That really helped me.

Samantha expresses confidence in her interest, competence, and performance throughout our discussion, but she specifically credits her environment for that extra support she needs; support she implies women at other schools may not be getting.

In contrast to Samantha's confidence in her environment and Hilary and Madelyn's confidence in their commitment, competence, and work ethic, Stella's considerable modesty helps her connect with others while majoring in astrophysics and 
advanced math at a large, public, research university in the mid-west, a path she admits makes it difficult to connect. At times Stella's modesty borders on language implying imposter syndrome, and yet further analysis reveals Stella's high competency and performance beliefs: "I do research and I think I have four-pointed every astro class I've taken. I understand it pretty quickly, I get the homework done pretty fast, and I understand the homework." And yet most of the time she doesn't express her science identity directly like this, rather she buries these statements within humble contexts such as the way she describes others' reaction to her double major:

When I tell people my major, I usually just say math, or I just say physics. They're kind of like, "Oh, that's cool," or "Wow, you're so smart." It depends on the person, I guess. I try to, not necessarily discount that, but I try to say, "No, I just really like it, I'm pretty good at it," then I try to pick something that they're really good at. Maybe I'm trying to make them not feel not smart, I guess. I don't like the description of: "Since you're in math or since you're in physics, you're so smart."

... I try to make it sound less smart than it is.

Stella's desire to connect with others despite the barrier her STEM majors create causes her to select when she will disclose only one of her majors to others. Further, Stella uses the interest and competency dimensions of her identity to connect with others when she says that she just "really like[s] it" and is "pretty good at it," and how she then works with others to identify their interests and areas of competence. Although she does not self-promote much, Stella's confidence is apparent when she describes how she approaches her studies, her level of engagement in her research and academic programs, and her confidence when interacting with her peers.

Recognition. The assured shared many examples of the various types of recognition they experience. Their ability to accept recognition in many forms, and to 
recognize their own competence and performance successes, sets them apart from participants in the other categories of experience. Furthermore, the assured articulate the ways recognition goes beyond their own success as acknowledged by those of authority, but also includes their reputation among their peers as successful and their sense of community within their major. For example, Madelyn described her experience with recognition in this way:

I've gotten comments from professors and from people, affirming the skills that are required to be a physics major. People always told me, "Oh, you're really good at problem-solving" or "You're really good at approaching a problem, and you're really creative with doing this and that." That's what you need to be a physics major. Yeah, people have commented that towards me, and it's kind of unusual, it's like, "Yeah, okay, I can do this." It makes me feel like I belong here.

In the way Madelyn and others in this group are willing to look beyond traditional types of recognition such as presenting research, being invited to serve as a teaching assistant or tutor, or winning an award for research or academic achievement, Stella shared many examples of these types of recognition she has received:

You're recognized in the sense that the students know you as the one who will answer the questions, or the students know you as the one who will ask interesting questions and not just silly ones like, "Is that h-bar, or is that just h?"

Stella's humility and reluctance to self-promote by using the second person in this example is apparent; however, her willingness to perceive recognition in various forms such as her reputation among her peers serves as a mechanism to further support and uphold her confidence in her competence and performance beliefs. 


\section{"Ideal" Congruence}

The assured identify ways in which they are fulfilling the "ideal" physics student expectations, such as Hilary's list of reasons why she considers herself the best student in her program. Additionally, those assured about their experiences as a physics major seek reconciliation when they may not be considered "ideal" by themselves or others.

For example Madelyn, who comes across as an unstoppable student and leader who enjoys her studies, her research, her campus involvements, and working with others, knows she doesn't fit the stereotype of the "ideal" and she's not bothered by this in the least. However, it's not the case that Madelyn has never had doubts:

I think I had a moment during my sophomore year where I felt like. . .I wasn't meant to be a physics major. . .Because I feel [sic] like you had to be naturally gifted at math and science. I grew into the community and I got more self-confidence in my ability so. . .I no longer feel that way. I just love the field for what it is now.

Madelyn cites grit as the source of her ability to work hard through these doubts or concerns about not fulfilling the stereotypes: "I realized later on that the one thing you need, whether or not you are naturally gifted in math and science, is having grit, and a lot of it." It's not surprising that Madelyn experienced this level of doubt in her sophomore year since it was around that time when she used to lie about her major after experiencing others' negative reactions when she disclosed her major, such as Madelyn's recounting of a faculty member's reaction: "Well, that's different. I've never seen a woman in STEM before." Madelyn's grit motivates her internally to strive for excellence in her studies and her research, and to push past external barriers:

When I was 15, I wanted to do astronomy, but I didn't know I could pursue a physics major, so I emailed this professor in [a large, research university]. I remember this like it was yesterday. I asked him, "How can I 
become an astronomer?" He told me, "Well, I mean, it's really hard. You can major in physics, but that's really, really hard. You should just take it as a hobby and not even try to pursue it." I'll never forget that. I think about it to this day. I remember how it made me feel like I wasn't good enough to do it since I was a girl. I wasn't equipped with the right mind for math and science. I actually look back and I'm like, "oh, he was totally wrong."

In contrast to Madelyn, it may or may not be the case that Hilary ever has doubts, but it never came up; she unapologetically exudes self-assurance. The remainder of the assured do experience doubts and setbacks from time to time, but others may not know this based on their descriptions of their competency levels and their performance. This may also be a result of another commonality across the experiences of the assured and the "ideal." Participants in this category of experience described a heightened awareness of perceptions of the "ideal" from within STEM in comparison to others outside of STEM. The assured don't often experience expectations of themselves compared with "ideal" stereotypes from others within their major, or at least not in ways that they perceive as detrimental. However, they do regularly experience perceptions of "ideal" incongruence from others outside of STEM. Samantha summed up the reactions of others in this way, "Most of the comments I get are surprise and shock. . . It's kind of weird."

Despite the consistent reactions of shock when they disclose their major to others outside of STEM, their grit and well-developed physics identities allow them to reconcile these experiences with relative ease, such as Madelyn's willingness to say that the professor she emailed was "totally wrong." In these ways, the assured are aware that some people believe that women do not fulfill the "ideal" of who is best suited for physics, but their assurance about their own abilities and interests compensates for any incongruence others might project onto them. 


\section{Gender Congruence}

Similar to perceptions and experiences with "ideal" expectations, the assured are aware of gendered expectations, gendered obstacles and disparities in their field; however, their experiences are not described in terms that indicate gender incongruence as a female in a male-dominated major. Madelyn's grit motivates her to push past barriers, and Samantha's women's college environment serve as examples of the different ways participants in this group have reconciled any incongruence and instead experience congruence. Awareness of the "chilly climates" and assumptions about the "ideals" within STEM resonate differently with the assured who may notice these potential barriers, but rarely describe themselves as being hurt or deterred by them. The assured also do not think gender makes a difference in their major, and they don't often notice a

difference when working in co-ed groups. Samantha simply stated, "I think anyone could do physics, if you put the time that it takes into it, it doesn't really matter what gender you are."

Perhaps Stella is subconsciously influenced by the way her major sets her apart as simultaneously not fulfilling the "ideal" scientist and the "ideal" female goal affordances discussed by Diekman et al. (2017). Through Stella's attempts to connect with others even when she interprets their reactions to her majors as a barrier to connect, she speaks with assurance when she discloses that she's, "never felt discriminated against for being a woman [by male peers or professors]."

Hilary knows that gendered biases exist in physics, but she cannot personally recall any examples when she's experienced this. Instead she seeks gendered reflections in this way: 
When I was doing my internship in [another country] last year, we were all of the students assigned mentors. Mine was a woman, and that was pretty cool because all of the other ones were men. There were not many women there. She worked with that observatory with her husband and their kids. It was just kind of like a really good thing for me to see that, yes, I can get married and have children, and still have a career as a professional astronomer, work in an observatory; I can have all of this at the same time. It's doable. It was really good to see an example of this woman not having to sacrifice one aspect of her life for her career.

Only Madelyn broached the topic of being bothered by interactions that could be motivated by unconscious bias, but then quickly reverted:

Madelyn: I have felt moments where it was just such an obvious answer that

like, their thinking that I was wrong could have possibly been just because I'm a woman. There's also a billion more times where it's just because none of us know what the answer is... [S]ometimes there is that gray area where I'm like, "Are you really just being stubborn because I'm a woman? Or are you just trying to find the right answer?" It's always a hit or miss.

Bronwen: When you notice things like that, and you feel like it is maybe because they're being stubborn, do you ever bring that up, or it's unsaid?

Madelyn: No. I usually never bring it up because it seems to be a sensitive

topic to people. I generally just let it go because it's not that big of a deal to begin with.

Bronwen: What does that mean that it's sensitive to people?

Madelyn: If I was to put myself in a conversation and be like, "Oh, you're not

doing this or you're not agreeing with me because I'm a woman," it's kind of like I'm pointing fingers at people when I don't have enough evidence to actually make that claim, so I'd rather not jump to conclusions that could be very wrong. They could be just as confused as I am. I don't jump to conclusions, so I just let it go, even if I assume that might be the case that they're being sexist. 
Similar to the others in this category of experience, Madelyn's grit and assurance allow her to move past visible and invisible forms of sexism quickly.

The way the assured navigate and reconcile gendered incongruence serves as another example of the precision with which the assured describe their willingness to take on responsibilities and to seek challenges outside of their comfort zones. The assured also experience their approach to reconciling incongruences by making what they describe as conscious choices to put aside their concerns related to sexism and gendered disparities in their majors and focus on the work, their success, and their own ambitions. This approach to norms and expectations in their major allows the assured to experience congruence between their identity and their experiences, creating a sense of resilience and confidence not experienced by some of the other categories.

\section{The Solitary}

Marked by high levels of interest and high levels of intrinsic agency, participants in this category of experience are independent individuals who appreciate and accept competition, practice pragmatic competency beliefs, and value communality only as far as it benefits them. Dakota's description of her experience in her major as "very solitary" unintentionally captures the congruence described by these participants who find the independence and individualistic features of the culture of physics reaffirms their solitary preferences and experiences.

\section{Identity Congruence}

The solitary experience congruent physics identities marked by their deep interest levels, their pragmatic performance and competence beliefs, and their limited experiences with recognition due to their insulated positioning. Despite this congruence experienced 
by the solitary, because of few recognition experiences and the resulting pragmatic competence and performance beliefs, their physics identity development is less defined than the assured.

Interest commitment. Dakota expressed early interest, "ever since I was really little, like in kindergarten when we would go around the classroom like, 'oh, what do you want to be?' And my dream was, 'I want to get my PhD.'” This was echoed by all participants in this category, with words like, "passion" and "enjoyment" and "fun" used often to describe how they experience the interest aspect of their physics identity. In fact, this deep sense of interest and enjoyment in their learning and research was continually cited by solitary participants as what motivates them in spite of few examples involving high competence or performance beliefs and recognition. Dakota said that her enjoyment of a very challenging research course that she had heard "horror stories" about was, "a good sign that I might be on the right path."

Pragmatic Performance Beliefs. Pragmatic to low competency beliefs appear to be normalized for this category more so than for other categories despite never expressing doubts about their major or career plans, which might instead be supported by their interest level. When asked directly, Dakota commented, "No, I never have second thoughts." The solitary question their abilities, but consistently share an awareness that this is common. And her graduating $3^{\text {rd }}$ in her high school class in high school, Dakota has experienced what might be considered the typical drop in grades in college STEM courses. This drop in grades, coupled with all exams and class grades posted, she now describes herself like this: "I'm pretty middle of the pack." 
Three of the five participants in this category are non-traditionally aged students who cite various reasons for performance issues either presently or in the past. Take Delores, for example, who has returned to complete a bachelor's degree in physics in her 60's after a bachelor's, master's and successful career as a dietician:

My brain doesn't work the same as when I was young. That's a big challenge because of my age. In fact, that's very frustrating for me because it takes me longer to remember something. I think that's the most frustrating thing, but then the professors don't let me have that excuse either, which is good and bad. I get frustrated that I can't keep up with the other students sometimes, but at the same time, I'm encouraged that they don't give me that excuse, because they have high expectations of me.

Brooklyn's experience is similar in that she is, "not blind to my obstacles. I also know that I won't be satisfied doing anything else. So why wouldn't I keep going?” Both Delores and Brooklyn have found the curriculum challenging, and their grades have reflected that at times, but they persist because they identify with the major regardless.

Recognition. This identity dimension serves a critical role for the solitary, as two out of five participants in this category had no examples of feeling recognized in their major whether asked for this directly or indirectly. A third participant in this category was able to identify an experience with recognition but only after considerable probing. Instead, most of their examples featured others being recognized by faculty and department administration when they felt that they were deserving, or that recognition was always reluctant. In lieu of some traditional or obvious forms of recognition, participants in this group utilized positive self-talk and identified other ways to recognize their own efforts. For example:

I think that there should be an award for people whose GPA has improved ... I felt very discouraged when I didn't get one of the academic awards at the awards thing. Even though I definitely know that my GPA didn't show 
that I could win this award. . .but then when I got hired [as a TA], it was saying that the faculty felt comfortable enough, more than comfortable. That they're going, "Hey, we trust you to teach."

This ability to recognize and evaluate their own performance and competence for themselves was consistent across the solitary group. This could be due to a combination of their individualized work style and position within their courses and research experiences.

\section{"Ideal" Congruence}

"I'm not a total genius. . . .At one point, when I was much younger, I thought, 'Well, I'm not bright enough to get a degree in physics."' The solitary often described acceptance of assumptions and stereotypes about who is ideally suited for physics. As exemplified by Delores' quote at the opening of this section, the non-traditionally aged students in this group in particular experienced concerns or doubts about being able to major in physics in earlier phases of their lives. Additionally, normalized assumptions about who is best suited for physics is congruent with normalized assumptions about gender. Solitary students often cited male peers as the best students in their classes, and equated speed and performance outcomes with the highest levels of success. Dakota shared a specific example of why she considers one of her peers to be fulfilling the ideal:

Like one guy who is not particularly good at physics concepts but gets really good grades because he's super good at math, and can do complex computations in his head, and he's just really fast at it; but, not necessarily great at physics concepts.

Despite experiencing acceptance of ideal expectations for others, participants in this group also experience acceptance and congruence through their awareness that they may not fulfill ideal stereotypes related to physics and gender. The solitary do not seek to 
reconcile what others might perceive as incongruence because they do not perceive or experience this personally. To understand this further, the next sections will explore in depth the gender congruence experienced by the solitary.

\section{Gender Congruence}

Although they are aware of sexism and gender disparity in their majors, the solitary are unbothered by this, believing: "It's definitely not based on gender at all. There's really, really successful girls and really, really successful guys." Matilda is not bothered by gender disparity in her major, "For me, [gender] wasn't a social impediment, really. I just sort of flew over it." However, from time to time she does feel guilty about not actively supporting the other woman in her class who is outspoken on the issue:

My reaction was a little weird because I wasn't involved in that stuff, so I felt like maybe I should be, but I didn't have the energy or interest to invest. I supported what she was saying, but I wasn't really backing her up actively any more than the other students. I felt a little guilty about that, but I don't have an obligation. . .

The physics department at [my school] is so small that you know everyone in it by the time you graduate. ... When you know everyone, it stops feeling like a bunch of men and it starts feeling like individuals, and that causes gender to fade into the background for me.

Instead of supporting the "active feminist" as she calls her peer, Matilda takes what she considers a more subtle approach to raising awareness about gender disparity in STEM:

If some girl entered the room who hangs out with one of the guys but isn't a physics major, I'd be like, "Wow, I'm not the only girl in the room anymore." Not in an aggressive way, but just making sure people noticed.

Although the solitary students all described a keen sense of the gendered issues faced by others in their field, one of the defining criteria placing these students in this category was their consistent descriptions of gender congruence for themselves personally, which often focused on a heightened sense of intrinsic agency. 
Well-developed intrinsic agency. Delores' assertion, "I prefer to work alone[,]" and Dakota's description of herself as the person who didn't want to be let down by her peers, so she would "take control of the whole project, and pretty much do the entire thing myself" epitomize the way participants in the solitary category of experience articulate high intrinsic agentic roles and goals marked by independence, individualism, and self-direction. The solitary prioritize their interests and needs in the present and in the future such as the way Brooklyn conceptualizes her future: "What I am super interested in is just diving into research. Even if it's stuff in a small basement room in a cold building by myself for hours, and hours, and hours." Dakota, who used the word "solitary" to describe the culture of her academic environment, utilizes independent, selfdirected terms to describe her work style, even when in a group:

I think I took a much stronger leadership position than [her male teammate] was expecting, because it was my project idea, and I had the whole idea for it in my head. So I was just kind of like, "Here, this is how this is going to work, and... the professor says it's ok and all good to go, so [pauses], here we go."

Dakota's willingness to self-direct their group project including concept development, discussing the proposal with the professor, and the way she shares this with her teammate illustrates the way the solitary prioritize independence and individualism, even in a team. Dakota, Delores, and Brooklyn, all articulated varying degrees of barriers to developing their social and professional networks in their major; their examples demonstrate how their well-defined intrinsic agency is integrated across their roles and goals.

Despite these examples of reluctance to work with others, participants in the solitary group can work in teams as they often must; some solitary participants even enjoy group work and organize it when working in teams will benefit them directly. 
Matilda described herself as an informal leader in her program and, "the face of the department," and studies with her peers regularly because: "Our problem sets were, they were just too difficult to do on your own." Beyond this necessity, Matilda articulates a number of examples of her working well with others as she does here:

I felt competent when I was working in the [study] group a lot. I felt like I tended to be more on top of the material than most of the students, and often I could help explain something to somebody else, and really liked trying out my explanation from different approaches so that in some ways they would understand and see it as I did, and see why it would work.

Matilda's example illustrates the enjoyment she experiences with her study group based on a mutually beneficial endeavor in which she gains, feels competent to contribute, respects others and feels respected. Gloria takes responsibility for organizing and facilitating regular study sessions because, "I was really worried about the Physics GRE." For both Matilda and Gloria, group organizing is motivated by their desire to succeed, not necessarily marked by their desire to connect with others personally.

Solitary students value, participate in, tolerate, or at minimum appreciate the motivating aspects of another of Diekman et al.'s dimensions of agency, competition. However, the motivating factors of competition can be limited when a student feels solitary and bears the pressure of that competition alone:

[T] he constant pressure for grades and how that's the whole focus, it's incredibly stressful, and not healthy. There's a feeling of, real or imagined, feeling of competition to compete with fellow classmates, and I think it probably comes from that encouragement to do solitary work.

It's important to note that Dakota described an environment that valued solitary work above and beyond any other participant in this strand of the study. For a student who already feels intrinsic agency at a level that might go beyond independence to resemble 
something more like academic isolation, Dakota does not articulate awareness that her solitary environment magnifies the pressure of competition that other participants in other environments find tolerable, and even motivating. Dakota's intense relationship with independence and individualism serves to clarify the way this category prioritizes intrinsic agency and simultaneously de-prioritizes communality.

Low communal goals and roles. Low levels of interest in communal efforts such as connecting with others, helping others, and serving their community contrasts the welldeveloped individualism, independence, and self-direction of the solitary. Both Brooklyn and Dakota had trouble building and maintaining a strong social and professional network through their academic endeavors, specifically citing the transitions from freshman to sophomore year, and sophomore to junior year, as detrimental to maintaining a consistent network: "[P]eople just wash out super-fast in the first few years, which means that whatever friend base you had is kind of like not secure." When probed to elaborate on why connecting with others can be challenging, Brooklyn cited competition for benefits beyond grades as among the barriers to her connecting with others and building her social and professional network:

I will say that as junior and senior year progressed, I was really uncomfortable with the competition sort of state of things. People got very competitive with brown-nosing and trying to sound smart around people who they thought mattered. It became more politics than it did people being interested in learning about the subject....That really kind of started breaking us apart. A lot of us ended up becoming closer friends with the geology department than the...astronomy and physics department - it's combined - because we were at each other's throats, trying to look good for everybody. I just find the whole thing silly, and I can't really compete with it because I'm not very good about bragging about myself. 
This low level of communality Brooklyn experiences is common among participants in the solitary group, and the difference within this group may be a product of their environment as much as it may be something they identify with personally and is therefore not fixed.

One of the ways some participants in this phase of the study articulated their gender roles and goals as congruent came from incorporating teaching in some ways into their future plans. Gloria elaborates on why teaching is not an option for her: "I'm terrible at teaching people. I don't have any patience for it at all and it just frustrates me and makes me angry when people don't understand things for the first time. I would be a terrible professor." Brooklyn agrees: "If it was minor teaching, I would be fine with that. I love spreading knowledge.” Teaching goals commonly motivated by a desire to help students learn and grow are absent for any related goals held by participants in the solitary category.

Of all the solitary participants, Matilda was most willing to articulate the communal goals of connecting with others:

I started [studying in a group] my second semester in electricity and magnetism. I didn't really need to, I was just doing it because it was more fun, a little bit faster and I could help the other people, and I liked the other people.

This level of communality could be influenced by the culture of Matilda's program. Just as Dakota's program promotes solitary work, which further highlights her own individualism and isolates her, Matilda's program promotes communality through daily hangouts in the department when most of the faculty and students take a break for a snack and fellowship. This level of connection between the students may encourage a sense of 
community, even among students for whom connecting is not as natural, making the daily group study sessions a natural extension of the community built by the department. And yet, in a detailed description of the way that study group works, again individualism and independence are prioritized rather than communality: "People would be working on their set alone or with one or two other people, and people were in different places working on different problems, so you'd work on it like that." And this is what Matilda had to say about those who don't hang out in the Physics Building or attend the regular study group sessions:

There were a few majors who just maintain other friend groups and aren't very present in the department, and sometimes show up to work on problem sets with the rest of the people and sometimes don't. They're kind of mysteries to the rest of us. We gossip about them. "Are they geniuses and figuring it all out on their own, or are they just turning in problem sets half complete?" We don't really know.

The solitary participants do not view their non-communal behavior as incongruent to society's expectations of them as women; rather they view their behaviors as congruent to their own sense of self and their science identities.

One of the distinct features differentiating the solitary from the other categories of experience is that they see other equality issues such as ageism, ableism, and intersectional LGBTQ related issues as more critical than gender equality alone. It is possible that interest and personal experience with these identities further marginalizes these participants beyond their underrepresented gender status, further amplifying their solitary pathways. For example, when Dakota was asked to present to the department about her takeaways from the CUWiP conference, this is how she experienced her 
decision to incorporate one of the conference speaker's expertise on LGBTQ members of the physics community:

Generally, it's a pretty accepting community, I like to think so anyway. . . . Well, I brought this up [in the presentation] and there was dead silence. Like, "Uh, why would you bring that up? That's a difficult topic." Like, capital letters, Difficult Topic.

Across these various experiences with gender and other identity issues, the solitary do not articulate incongruent experiences between their gender roles and goals, therefore neither do they articulate any related dissonance. They all described their academic work as "fun" and expressed sincere interest in persisting in a STEM field as important to their career plans. Therefore the well-developed interest dimension of their physics identity and their well-developed dimensions of intrinsic agentic roles and goals guide them. This category of experience confirms previous research findings that, "the scientist is characterized as a person who prefers 'to be left to himself, to be left alone with his mind and his books"' (Parsons, 1997, p. 758) which has been confirmed since (Diekman et al., 2010; Hazari et al., 2010).

\section{The Communal}

Marked by language distinctly expressing thoughts and behaviors embodying the dimensions of Diekman et al.'s (2010) communal gender roles and goals, participants in this category of experience have relationships with the conceptual framework that experience incongruence when communality is not afforded, and anticipate future goals marked by communal consistency and congruence. Prioritizing communality influences the way participants in this category seek and maintain this congruence, accepting that this may require career plans outside of the straightforward set paths the assured and 
solitary participants navigate. As Diekman et al. (2010) stated in their discussion on communal motivations and why women opt out of STEM careers: "It is ironic that STEM fields hold the key to helping many people, but are commonly regarded as antithetical (or, at best, irrelevant) to such communal goals" (p. 1056). The discussion goes on to state: "Indeed, science-related fields with the greatest influx of women are those that are most obviously involved with helping people such as psychological science and the biomedical sciences" (Snyder et al., 2009 in Diekman et al., 2010, p. 1056). Naomi, a physics and mathematics major at a small HBCU in the south shares this sentiment: "My passion is always helping others, I love helping others. . Whether it be math, physics, or bio-medical science, I have that background that I can put forth some use in helping someone." Helping others, a dimension of communal gender roles and goals in Diekman et al.'s work, is among the critical relationships of this category of experience.

Experiences and goals for participants in this category most closely mirror Diekman et al.'s (2017) general model of the goal congruity process, which posits that past experiences and anticipated (in)congruity shape future goals and roles sought by women. For example, Naomi wants to pursue a Master of Business Administration (MBA) after graduating with her bachelor's degree in physics. Gabriela has enrolled in a master's program focused on medical physics so that she can help people in more "tangible" ways. Marion's plans are the most traditional, as she is pursuing a masters in physics and eventually a $\mathrm{PhD}$ in physics, but as will be discussed, this is due to her highly communal environment. This goal congruity, either through communal career choices or environments that make physics appear more communal, afford each participant a 
concrete path to address anticipated incongruence as they seek and prioritize communal congruence.

\section{Identity Incongruence}

For communal participants, their physics identity is experienced through communal connections. Each dimension of identity for participants in this category tends to be developed through others, takes place outside of physics spaces, or is motivated and maintained by communal dimensions such as helping others, connecting to and serving others, and help from others. The way identity is framed through communality for this category stands in stark contrast to the assured and the solitary whose physics identities are more clearly defined and independent of their experiences with gender roles and goals.

Interest. Although each participant in the communal category expressed interest in their physics major, these interests were attributed to chance and the influence of others, rather than something developed early in their lives. For example, Marion uses words like "fun" and "exciting" to describe her major, but her interests always tie back to communal goals such as when she said, "I have a lot of very flighty sounding things about why I am excited about physics, and of course, at the core of them all is because it's furthering human knowledge." And: "It's just a terrific network of people and a fun curriculum of course. The subject matter was always exciting, but the people really made it." In comparison, Naomi and Gabriella expressed low levels of interest in physics. When asked to share a time when she was most interested in physics, Naomi stated she could not think of an example, and instead said, "I don't think it would be one of my physics classes, I would have to say it would have been during one of my organic 
chemistry labs, or even just in chemistry lab altogether.” And although Gabriella was more clear about her interest when she stated, "I like what I do and I really work hard at it[,]" she went on to state later, "all the students in my year were really good and supportive.

Competence and Performance. Similarly, physics identity dimensions associated with competence and performance were muddled, and even disrupted, at times. Participants in this category of experience shared an unwillingness to discuss their competence and performance directly, or with confidence. Gabriella said, "it's not very natural for me. . .I can definitely work hard at some things, that's the best I can do.” In another example, Naomi discussed competence in other skill sets rather than in physics:

I'm really big on writing. I would study what they were doing and I would write it as we go along, I really love writing even though, probably, I'm as good at math, my strength is writing. I love to write. I would say, "All right, all of the writing, all of the lab reports, that's mine. All of the figuring out the numbers for the reports, I would do that."

Although Marion does not cite other skill sets as those she feels more competent in, she does cite grades as evidence that she is what she describes as, "aggressively average." This aspect of Marion's physics identity appears disrupted because later in the interview she discusses the reasons why she was accepted to a master's in physics program and promised a spot in the $\mathrm{PhD}$ program at the same institution if she passed the qualifying exam at the end of the year; she states clearly that her grades were good enough to get into the $\mathrm{PhD}$ program, but that they could not accept her because she was missing a prerequisite exam.

Each of the communal participants were reluctant to discuss their competence and performance in clear terms throughout the interview. The few times when they were more 
direct, as we saw with Marion's description of herself as aggressively average, they were often unaware, or unwilling to give themselves more credit for their own hard work and success.

Recognition. Communal participants framed nearly all of their examples of recognition by communal dimensions, such as helping others, or help from others. Furthermore, the communal participants identified recognition they received that was not specifically performance-based.

Naomi's passion for helping others motivates her inside and outside of the classroom, as evidenced by nearly every example she shared throughout the interview being framed in this way. Across the times when she felt recognized in her major, helping others was always the focus: "I don't know which professor it was, but they directed two students to me, for me to help them out in math. They trusted me enough to send somebody to seek help from me."

Marion often cites recognition of others through coursework and research when we explored this topic. Additionally, the clearest example she shared was not related to her competence or performance in physics:

One of our professors works on the long-range plans in nuclear physics for the Department of Energy. He helps write it and put it together and get it ready for publication. . . someone in the Department of Energy, puts a photo collage together for the back cover. When it was published our Department of Energy professor came in with it and said, "If any of you are interested, I'm working on the long-range nuclear physics plan and our very own student's on the back here." They had included my picture on the collage. And he was just so excited to tell people I made it onto the long-range nuclear physics plan. 
Although Marion sees it as an honorable form of recognition for her photo to be featured on the back cover of the long-range physics plan, it is interesting that this is her only example of recognition that is solely about her throughout the interview.

Gabriella also did not have many examples of recognition relating to her performance, stating that the time she felt most recognized was when a research mentor told her that he enjoyed working with her; recognition that is again framed in communal terms. A more traditional interpretation of Gabriella's assertion might find her desire for likability antithetical to her competence beliefs, but this incongruence is explored in depth by Diekman's work, resonating with Gabriella's goal affordances and social role selections as she makes her future education and career plans.

Beyond that example, Gabriella discussed negative experiences with recognition in the form of benevolent sexism such as the time someone said to her, "All the guys you'll work with will want to take you out. There aren't many women here." And her internal response to this was, "Really, you're telling me this now? It's just silly."

The only time I've felt a little, I don't know if I've felt discouraged, but a little bit "cute-ified" or something. . I was shadowing someone who worked at NIST - National Institute of Standards in Technology. It seemed like everyone who worked there was an older man, so everyone I got introduced to, they didn't say it with words, but it just felt like they were like, "Aww, you want to be an engineer? That's cute."

Recognition has been found to be a significant predictor of female physics students' persistence, and therefore it may not be surprising that participants in this group experienced little, negative, or unclear, examples of recognition. This coupled with their highly communal orientation influences the way each participant in this category makes plans to persist in the future. 


\section{“Ideal" Incongruence}

Perceptions and assumptions about the "ideal" physics student did not emerge as clearly for this category as it did for the assured and the solitary. Perhaps this is due to communal participants' orientation towards communal goals and roles which are assumed to position them incongruently with the ideal expectations. Furthermore, others' reactions to this incongruence appears to influence their comfort in physics spaces and their willingness to make plans to pursue the path of physicist. For example, when discussing the behaviors and characteristics of the "ideal" physics students, Naomi was unwilling to identify an exceptional student in her class: "I wouldn't say that we have too many that stand out because we all work together - it would be a collective. . We don't try to outshine one another." This highly collaborative perspective is specific to this category and was not discussed by participants in other categories when describing physics culture. However, Marion used the term multiple times, and when she reflected on the external perceptions of her major in comparison to her own description, again she solicits the term collaborative as what best describes her major in her experience:

I do get the impression that [others think physicists are] somewhat isolated and - not introverted, but you tend to work by yourself, and it's all theory and formulas and - there's no real collaboration. But in reality it is almost 100 percent collaboration.

Marion elaborates further on other's assumptions about physics and the way she conceptualizes her own position within this ideal:

It's kind of fun, honestly, it's a boost to the self-esteem when people go, "Oh, that's an impressive major." And you go, "Oh. It kind of is, thanks.". . . Most of the time I'm inclined to tell them, "No, it's not the Einsteins and all of that. It's more struggling through homework at 2:00 in the morning." But the perception is nice. 
For that moment, Marion acknowledged the status of her physics major and her own satisfaction in this status. For the remainder of the interview however, her experiences were framed in communal terms at all times.

Gabriella's poignant example summarizes the reasons why she believes she is perceived as incongruous to the ideal: "I'm a woman who cares about how I dress and I wear make-up, and I think people are surprised about that. Superficially, I don't fit their stereotype of physics majors.” Beyond the superficial incongruence Gabriella experiences, the assumptions and stereotypes about the ideal position participants in this category as inherently incongruent due to their highly communal orientation. The following sections will elaborate on the way participants in this group seek reconciliation and congruence as scientists and as women.

\section{Gender Incongruence}

Two of the three participants in this category of experience scored very low on extrinsic agency, low on intrinsic agency, and very high on communality; the third participant in this group scored high across the three gender roles on the CUWiP survey. Despite some variation in their gender role scores, participants in this group focused their examples and goals on communal dimensions throughout these interviews.

Helping others (tangibly). For the participants in this group, communality is valued, and for Gabriella and Naomi in particular, the communal dimension of helping others is among their top priorities; both participants seek to reconcile perceived incongruity through their future plans. Gabriella has strategically sought a graduate degree program that allows her to build on her physics foundation while helping others: 
In my junior year I was trying to figure out where I wanted to go with my degree and I was shadowing a few people. I shadowed a medical physicist at the hospital ... and I just really like it and thought it was a great way to use science to help people tangibly, to see it immediately.

When asked if remaining on a more traditional physics career path, including a $\mathrm{PhD}$ and research, could help people in the same way, Gabriella reiterated her goal to know when and how she's helping others: "Yeah, I think, of course, people can advance knowledge in society by doing research as well. I decided that it was more for me to do something hands-on." Even within a research context, Gabriella frames her outcomes as helpful to others: "I did a couple summers of research and I was proud of what the outcome was. Both times I was able to do something that felt substantial to me and seemed to be helpful to my professors."

Naomi's desire to help people tangibly is voiced as clearly as Gabriella's, but has her setting goals that take her away from the path of a physicist to an MBA degree in order to start a non-profit organization:

I will most definitely branch--well not branch off--but tie my bachelor's degree and my MBA in to kind of open up a facility; open up a non-profit organization and have it where I not only help the homeless but also helping them get them back on their feet.

This goal to utilize her problem solving and critical thinking skills developed through her physics major as a foundation to create an organization focused on helping others and serving the community might appear tangential to some, but according to Diekman et al. (2017), Naomi may be setting goals in accordance to a construct of goal affordances which serves as the foundational belief and subsequent selection of social roles that, "afford or impede valued goals" such as communality or agency (p.21). Despite describing the culture of the physics department, faculty, and students at her university as 
highly collaborative and team-oriented across all contexts, Naomi seeks goals that will further align her skills, experiences, and communal values in ways that are unmistakable to her and others. Naomi knows this transition will require strategic planning, therefore she is tapping into and building her network to accommodate her goal affordances: "I'm currently reaching out to people that have started where I started from."

Connecting with others. The three participants in the communal category of experience clearly articulated robust forms of connecting with others through their coursework, research, campus involvements, network building, and social outlets. Marion reflects often on the support network she experiences in her academic program, and appreciates this aspect of her major so much that she has opted to continue her graduate studies at the same school in order to continue to enjoy the quality relationships she has built throughout her undergraduate experience. Among her many examples, perhaps that of the two female faculty in her physics department hosting bi-semesterly dinners at their homes for the female undergraduate and graduate physics students is the most poignant. At these informal dinners, discussion topics include research, current and future goals, and enjoying each other's company: “It was just nice. It really fostered a great sense of camaraderie." Beyond enjoying company and connecting, the hosting professors would take time to speak with every student who attended to, "make sure that we were getting everything we needed to be successful." In lieu of a sense of intrinsic agency, these opportunities to connect with peers and the faculty in an informal, supportive environment provide Marion with an external, communally-focused source of agency: "[I]t was very comforting to go into one of those dinners and know that if you 
were having a problem and wanted to casually, conversationally talk about it, that it was probably going to get resolved."

Attending to others. Power is an extrinsic form of agency that can be challenging for some traditionally aged college women in leadership positions. In Gabriella's example as president of a physics club on her campus, by her description, she was presenting competent leadership for the group and making sound decisions across a number of team projects; at one point Gabriella described herself as the "negotiator" for the team. However, Gabriella's communal values, especially her desire to attend to others, superseded her value for her position of leadership. Gabriella described feeling pressure in the leadership position after hearing that a group member - who was slated to succeed her as president - was unhappy with a decision she had made, which caused her to step down prematurely and remove her activity from the group permanently:

Gabriella: I found out from another member that she wasn't coming [to club meetings] because she was mad, so I decided that I would let her take leadership from that point so she could do it the way she wanted to.

Bronwen: How did she receive that news that you made that decision?

Gabriella: I didn't tell her that's why I left. I just told her that I was busy and I thought it would be a good time to transition while I was still there and she could ask me questions about how to do things. It wasn't an angry transition, she didn't know that's why.

In this scenario, Gabriella is unconsciously attending to her peer's feelings as a higher priority than her own feelings and desires to lead the group. According to the framework provided by Diekman et al. (2017), Gabriella is self-selecting a social role she perceives 
to afford her more congruence with her communal goals and values, which includes attending to others.

Across Gabriella's leadership example, Marion's strong sense of community within her program, and Naomi's goals to open a homeless shelter, we see three distinct examples of how highly communal undergraduate female physics majors will seek reconciliation of their incongruent identities, "ideal" perceptions, and gender roles.

\section{The Reflective}

Of the 18 participants in the qualitative strand of this study, a category of participants who articulated relationships marked by acute awareness of gendered roles and goals for women in STEM as described by Diekman et al. $(2010,2017)$ emerged. In addition to their conscious navigation of gendered expectations, these students are equally reflective about how they navigate assumptions about the "ideal" physics student. It's not the case that this group experiences gender-based bias, pressures, or sexism more than participants in other category of experiences - in fact, two of the four participants in this group articulated their own version of "I've not experienced this personally, therefore I'm not bothered by this directly,"- but, the ways that they manage and articulate their own assumptions, and are critical of the assumptions of others, about gender and the "ideal" manifests differently from other participants in this study.

\section{Identity Incongruence}

Interest, competence, and performance beliefs. Each of these dimensions of a physics identity emerged slowly for the reflective participants. Many of them did not experience authentic interest in their physics major until they were in college, or after completing a bachelor's degree in another field. This level of interest also impacts 
participants' willingness to articulate clear goals for the future; as Yvette stated: "For right now, I really just want to get some experience. I don't really know what my dream job would be. I'm just searching." And Dahlia places emphasis on her many interests prior to her declaration of a physics major, and her persistent interests outside of this major. Since she wasn't fixing or building things growing up as she perceives her peers were, Dahlia sees her interest in physics as secondary to her willingness to work hard and build goals that satisfy expectations she attributes to her parents and her culture. Some participants in this group found their interest in seemingly whimsical ways, such as Vanessa's assertion:

I wanted to do a double major in math, because of my previous degree, I didn't have to take any liberal arts classes really, and they encouraged me to do physics instead. So I was like, "Alright, I'll try physics, what the hell?" And I ended up loving it.

Beyond their interest in physics, reflective participants connect their competence and performance beliefs to experience and expectations. Participants often cited completing the work as evidence of their competence; this made them proud rather than the grades they received.

Recognition. The recognition dimension of physics identity is the piece that truly sets the reflective participants apart from the other categories of experience. Many of their examples of recognition were negative forms of recognition. The reflective consider the subtle ways gender is performed in their majors through their examples of recognition, such as when Carly noticed in lab settings that, "the guys would look at each other when they were talking. . .it was hard getting their attention and even eye contact 
sometimes was difficult." Vanessa has also observed recognition in the classroom to be gender-based:

So this professor, when we were in our very first lab, he went around and checked the circuits of all of the groups, and only my group had women in it, me and my friend. And when [other groups'] circuits didn't work, he assumed that it was the equipment, like the philoscope wasn't working right, the signal generator wasn't hooked up to the wall correctly. Only our circuit, did he check the circuit to make sure that we had put everything in the right place and that everything was connected. Only ours. Which was infuriating.

Vanessa goes on to say that this professor nominated her for an award; however, in both of these examples, the level of detail to which the reflective participants are attuned when it comes to gender is clear through their examples of recognition. Beyond their observations, another notable feature common to participants in this category is their reaction, which ranged from the frustration articulated by Carly to the anger articulated by Vanessa. Participants in other category of experiences have observed gender disparity and discrimination, but how the reflective articulate the emotional impact these scenarios have on them sets them apart from their peers. Dahlia stated, "many of my women friends did not get the same recognition as our male peers, even though they ended up being good [in physics]." The perceptive nature of the reflective plays an important part in how they reconcile "ideal" expectations and gender and goal affordance in their studies and research environments.

\section{“Ideal" Incongruence}

Beyond being highly conscious when gendered roles and goals are performed, the reflective are also articulate and highly aware of the stereotypically "ideal" physics student assumptions and roles. For example, Yvette feels gender and looks play a part in 
the surprised reactions she always receives from others when she discloses her engineering physics and mathematics majors: "In a female, I don't really look like the stereotypical physicist, whatever that is." Carly echoed this when she recalled that she has often been told, “[y]ou just don't look like a physics major." Furthermore, Yvette experiences this surprise from people both in her major, as well as those outside of her major, such as when she disclosed her major during an introductory class meeting of a non-science class that was comprised of female students only: "Once I said my major, everyone got like dead quiet and looked around really awkwardly...It was really weird." Amply aware that she does not fit the ideal within her major, and does not fulfil the ideal female to others outside of her major, "awkward" and "weird" are just a few of the words she and other reflective participants use to describe their relationship with incongruent "ideal" scientist stereotypes.

Reflective participants are also often bothered by "ideal” assumptions and gendered roles; they articulate an active decision-making process when faced with these issues in context, choosing quickly whether to reject, ignore, or address the issues as they arise. Vanessa summarizes her reaction to a professor's assertion of the "ideal" by way of rejecting the notion altogether:

I was actually talking to a female physics professor, the one at our school, and she said to me, "If you ever struggle with a problem, or you can't figure it out, you're one of these people who can't figure it out, then maybe you shouldn't be a physics major." And I thought, "You are a bitch." I'm sorry for my language, but I was furious because, it really made me mad, because of course I struggle, it's never easy. And I don't think that because I struggle with it, that doesn't make me. . .that's like the typical stereotype, that you have to be a genius to be a physics major, and I simply don't think that's true. 
Although Vanessa rejects this notion, she is disturbed by the fact that the only female physics professor at her institution reinforces unrealistic stereotypes about who is best suited for physics.

In addition to their observations of external expectations for themselves and others, and their emotive manner of processing this information, reflective participants also consider the way they themselves perpetuate stereotypes about the "ideal" in their majors. For example, Dahlia's opinions on this topic have evolved through the years:

When I was younger, it made me feel a little bit special, you know, like I'm smart or better. But getting older, reading and learning more, has made me realize that's not such a good way to think about your field. It puts a lot of people off that I'm in this field, so I try to make it sound normal, because it is...A lot of it is what you learn, how much you invest and put in, and- Yeah it really is how much you work and, I think that saying it's something innate makes it very unhelpful for people.

Although not yet articulating a developmental stage in which they see themselves as ambassadors for their chosen fields, the reflective do see themselves as willing to contribute to their field beyond their science. Despite being acutely aware of the roles, goals, and potential barriers she may face in her future career, Dahlia does consider the significance of her identities as she makes plans to continue on her path towards becoming a physicist: "As a woman, and a person of color, I'm maybe one more person." Some of these participants may develop beyond this acute level of awareness in the future by processing their reactions to their observations and roles in different ways, or building more robust behaviors grounded in agency to address the bias-based behaviors they observe. However, it is important to note that at no time did members of the reflective group articulate a desire to move beyond this acute level of awareness into actions that would encourage different outcomes in the future. It should not be assumed that 
development beyond the unique characteristics of this category of understanding is required, or desired, in order for any individual participant to be successful in a maledominated STEM field.

\section{Gender Incongruence}

Marked by a comparatively heightened awareness about gendered roles and goals, participants in the reflective group articulated examples early and often throughout the interviews of when and how they see gender playing out in their classroom and lab experiences. For example, Dahlia's awareness level causes her to practice this: "Anytime I walk into a room, I just do an instinctive count of how many women are in the room." Beyond the gendered examples shared by participants in other categories of experience, reflective participants articulated a heightened awareness about the nuances of these examples, and the way they can influence women and other minorities trying to enter and remain in physical science majors.

Agentic gendered reflections. Specific to the reflective group, participants value communal experiences, and are challenged by experiences that require both extrinsic and intrinsic agency. The reflective group did not articulate feeling powerful, or having full agency, to resolve the gendered issues they observed on their own. In the examples shared, participants in this category practice agency in that they will notify someone else of the discriminatory behaviors they observe; however, they almost always cede power to someone in a position of authority, such as a professor, department chair, or even a graduate student. The reflective participants shared a number of related scenarios that were ultimately resolved, or left unresolved, by these others. At times, even this level of agency - sharing their observations with someone in a position of power who might have 
more influence - is seen as out of reach. For example, when Dahlia was asked if she had ever shared her observations about the culture of the physics department at her school which she described as highly competitive, full of "drama," and "toxic" - with someone in a position to influence that culture, she responded:

I've talked to [some older grad students] just to kind of vent, but I've never talked to the professor, no. . .I don't want to necessarily influence the production of either me or the graduate students. . .Although, I do talk to him when I have plans about different things and then about my life. I try not to bring his other students [into it] because that's just tricky territory.

Vanessa had an experience at her internship with another student colleague she labeled a "jerk," but after speaking with her internship supervisor about it, she reflected on the experience differently. Her supervisor explained to her that there is a place at the company for someone like him, and a place at the company for someone like her, and then he "showed" her an example of how to manage someone like her colleague the next time that student interrupted the supervisor during a team meeting. This resolution was satisfactory to her, and Vanessa felt equipped to address similar behavior and feelings in the future, despite a lack of actual resolution in that example. Here's how it went when she tried to apply her supervisor's advice:

Like, one time [the other male student intern] didn't believe me that I knew what the adapter for the computer looked like. Like a VGA versus an HDMI. And he made me show him. So after that I talked to him, I said, "Look, I don't care what your opinion of me is, we have to work together, which means we must treat each other with respect, and you're not treating me with respect." And he was like, "Oh well, I'm sorry if you're reading something into what I'm saying that isn't there." And I was like, "Well, you know, if it's inadvertent, I apologize for bringing this up, but I'm going to be honest on how you're making me feel." It didn't get better after that[.] 
Vanessa's example echoes Madelyn's observation shared during the assured category of experience discussion that confronting these concerns directly can be "sensitive" to people, particularly male students who are not consciously performing sexist behaviors. When Carly served as the TA for an introductory physics course, the female professor decided to spend the first few class meetings discussing diversity in STEM and disparity in physics for women and other minorities. This approach was received positively by Carly at first. After overhearing one of the groups discussing in a loud manner their opinion that, "there's quotas for women, and non-white people have to be in, and they are taking spots away from people that might actually be eligible." Carly shared this example with her professor because, "just from looking at some of [the other students'] reactions. . .It was just really obvious that there are some people who are uncomfortable and they are hurt. . [by] having their peers tell you that you don't belong here." However, Carly was met with what she considered an unsatisfactory resolution. Her professor highlighted all of the positive discussions that took place during those class meetings and did not highlight, or to Carly's knowledge follow up on, the discussion Carly overheard. This lack of resolution shaped Carly's opinion about the activity overall: "I really liked that she did that, but I just don't think...it was executed very well." In the example where Carly noticed that the males in her group were not making eye contact with her while they were working, or they would interrupt her when she spoke, I asked her if she ever brought this up to others in the group. She said she didn't have to because the professor noticed the behavior in a number of groups and made an announcement to the class: "Really pay attention to who's in your group who is talking most. . .and why that person was getting privilege to talk when other people weren't 
getting privilege to talk." Carly was glad that the professor addressed the issue this way. However, Carly noticed that the effects were brief, "I could see that they were trying a little bit to acknowledge everybody but then it went right back to how it was." After experiencing frustration and doubts about her competency beliefs, Carly opted to work alone to finish the project, her rationale being, "they are not listening to me, I am not going to waste my time trying to get their attention. I am going to try and learn something and work." This level of reflection, and the way Carly relies on her professor to resolve her concerns ultimately weighs on her emotionally, and in this case, impacts her ability to work with her group to complete the lab.

There were less-ambiguous examples when ceding power to those in authority positions seemed like the only option, such as when Vanessa gave her phone number to a post-doc thinking they could discuss physics and research opportunities. The post-doc began text messaging her with what she considered inappropriate frequency and content. She approached the department chair about her concerns after the post-doc told her she looked "sexy" one day. She knows that the department chair spoke with the post-doc because the post-doc has never contacted or spoken to her again.

Ranging from resolved to unresolved, participants in the reflective category are acutely aware of the nuanced gender roles performed in their physics spaces, and their approach to seeking resolution is to cede this power to others whom they perceive to have the position and ability to be responsible for the resolution. Even when these participants attempt to take a more active role in resolving the issues, there is often someone else involved, or in the position of power to ultimately decide the outcome. These participants do not hold a relationship with agency that allows them to personally resolve 
an observation or scenario of gender-based bias, often choosing to let the observed behavior go unresolved.

Communal gendered reflections. Students in this category of experience also report positive experiences with gender in their major; just as reflective participants know unsupportive or sexist scenarios when they observe them, they also know supportive, respectful, collaborative relationships when they experience them. For example, Yvette recalled one of her lab partners, another female, was her favorite because throughout the year they, "would just kind of work together the whole time. We didn't really split it up. We just worked on everything at the same time together." This level of collaboration might be viewed as inefficient by some, but for Yvette and her partner, this provided them with confidence and ensured that they both learned every skill and theoretical aspect of each lab, leaving neither to outshine nor fall behind the other: "If we didn't know what we were doing, we felt comfortable asking each other...and asking questions to the professor." Dahlia feels similarly about her friend group:

[F]ifty percent of what we talk about is women in science... And the other fifty percent is science. Okay, that's an exaggeration. We talk about other stuff too, like friends and life and stuff. The two main topics outside of your own everyday life, is like physics itself, and being a woman in physics.

This connection Dahlia feels with her friends who care about both science and gender is reinforced later when she shared her reaction to the actions taken by someone in her lab in response to the public comments of Nobel Prize winning scientist, Tim Hunt, that "girls" don't belong in the lab because, "you fall in love with them, they fall in love with you, and when you criticize them they cry." A male member of her research lab posted a sign on the entrance to the lab with a tongue-in-cheek response to Tim Hunt's quote, a 
defiant statement that encouraged Dahlia to reflect further: "I was really comforted, and in the end it made me feel like, 'okay, I can stay in physics with these people. Like, this is a community I can bear to be a part of."”

In an example of the way professors can show their support for women in physics, Vanessa shared that role modeling and taking an active role in educating themselves sends strong messages to students; messages that are resonating loud and clear:

I think the head of the physics department, he's the one that encouraged me to go to CUWiP my first year, and provided funding, he knows there's a problem; he wants more women and minorities in the major and as professors. I think he's acting to fix the problem. He went with us to CUWiP last year....He was like, "It was a huge change, I have learned so much about how women feel in physics, and minorities." And next year he's going to send another professor to go with us, just so that the professors are more aware of the issues.

In addition to the acute awareness the reflective participants have about gendered roles and goals in their physics majors, they are also acutely aware of those who are leading the change, and what behavior supportive of change looks like.

\section{The Ambassadors}

A Google search for the definition of the word "ambassador" yields: "A person who acts as a representative or promoter of a specified industry." This definition summarizes the interests and willingness of the two participants in this category of experience well. This category of experience is presented last because of its complexity and inclusivity of the hierarchical comparative presentation of the previous categories. For example, the ambassadors are acutely aware of the stereotypically "ideal" scientist and the incongruence in the fact that they don't fit this mold; but, their relationship with this concept is to actively pioneer their own definition and be the "ideal" scientist. Due 
to their awareness and ability to connect with different types of women in STEM, both participants in this category of experience clearly articulated goals to serve as ambassadors for STEM, particularly in the recruitment and retention of women in STEM, throughout their careers. The data indicate that both participants in this category fulfill this role in part because they share many characteristics across the five categories of experiences previously discussed, making their efforts to connect with and mentor other women in STEM plausible.

\section{Identity Congruence}

Despite occasional doubts, like the assured, Bethany and Stephanie articulate high performance beliefs, grit, and confidence in their chosen paths. In addition to sharing many experiences with the assured when it comes to their physics identity, participants in this category of experience also see their ambassador identity as seamlessly integrated into their physics identity.

Stephanie and Bethany's goals of supporting recruitment and retention efforts in physics appear to be important to them over the course of their lifetime. Bethany stated:

I like volunteering and helping other young females, and of course STEM majors, and maybe helping them choose STEM. . I want to be encouraging for other people. I'd like to do some sort of outreach project like that throughout my life.

Stephanie has concrete strategies already in place that guide her ability to serve as a selfproclaimed woman of color physics ambassador:

I'm trying to be openly geeky about my major. . .Anyone in a physics class loves that stuff. They have so much passion about it and they're so happy to be there that I want to share that with everyone else. I will talk to anyone. If we get on a subject that I don't know anything about it and I can tie it back to Physics, I definitely will. 
This example not only highlights Stephanie's goal to serve as an ambassador for her field, but also indicates her high level of interest; she is also willing to recognize physics potential in others through these conversations, which takes her beyond an identity development phase in which she is only requiring recognition from others.

Another experience both ambassadors felt confident about in terms of their identity development is the positive impact their experience at the CUWiPs has had on them. For both participants in this category, professional development opportunities such as CUWiP serve as recognition and support their ability to persist in their majors, and their ability to see their responsibility in contributing to the recruitment and the retention of women in physics now in the future. Bethany noted that in lieu of well-known successful female physicists who can serve as role models at her own university, the opportunity to meet the other women at CUWiP allowed her to see them as her inspirational role models, rather than only looking to male role models she had access to at her own campus, or seeing nothing when she looked for role models. Bethany said that it was, "nice to see that in my peers, and also see that in my university." Feeling this sense of camaraderie extend across their field provides Bethany and Stephanie with a "big-picture" view of their field, which allows them to seek role models and forms of recognition in what others might deem unlikely places. These experiences also allow ambassador participants to articulate what membership in the community is like to other physics students, and to people outside the major. For their ability to see beyond their own performance, or even the culture of their individual academic program, and their ability to relate to and connect with a variety of individuals, Bethany and Stephanie's experiences at CUWiP support their goals to serve as ambassadors throughout their 
careers and their concrete career goals which include R\&D in industry and a PhD in astrophysics.

\section{Redefining "Ideal"}

I'm Black, and I'm a female, and I'm an Engineer, and I'm in Physics. I'm combating everything right now. I'm the one percent outlier. I try to be as cognizant as possible just because of how people may view me because of that; how they may view that as a weakness even though I don't view that as a weakness at all. I'm just constantly aware of the fact that I will probably be the only female in this lab group. I'm constantly aware that I might be the only Black person in this class, or that if I have an opinion and it has anything to do with socioeconomical [sic] status, I'm going to be looked at funny; trying to be more than that girl and definitely trying to be more than just that Black girl.

Stephanie articulated her awareness that the "ideal" scientist stereotypically looks like someone else and possesses a level of brilliance and skill typically reserved for someone else who looks and acts "more" like a scientist. Bethany has experienced this also: "[S]ome people are very rude about it and they'll say like, 'Oh, you're a girl studying physics?' Or like, 'You dress too nice,' or, 'You're too pretty to do physics.'” Bethany goes on to call this a "restricted" view of physics and other science majors in the US. Reactions like this have caused Bethany to feel embarrassed at times to disclose her major to others, "I don't want to state it proudly all the time. Which is weird because it's my major, right? It defines a lot of my life." This fear of judgment influences whether or not she feels embarrassed to disclose her major especially when performing stereotypically feminine activities such as getting a manicure. Stephanie echoes the way assumptions about the "ideal," particularly from those outside of STEM, influences how she understands this restricted view of physicists and engineers: "I feel like it is so far away from what is normally expected of people that their reaction is astonishment; but 
it's like, is that a bad thing?" Despite feeling badly about those reactions from time to time, Bethany is quick to share examples of comments that are more aligned with the way she sees her role in the field such as, "you're helping even out the gender gap in physics and STEM." And, "you must be a hard worker."

The ability to question assumptions about the "ideal" affords Stephanie the opportunity to examine the stereotypes, incorporate what works for her, and reject what does not. For example:

[I] make sure that I'm not in the bottom percent of anything just because I don't want to give any fuel to that fire. If I am, I try not to be as hard on myself and try to remind myself that I am dealing with a lot more than what that guy over there is dealing with.

Understanding the intricacies of the "ideal," questioning it, at times rejecting it, and utilizing selected aspects to motivate and inform allows both Stephanie and Bethany to redefine the "ideal" for others in non-disparaging ways. Consider the way Stephanie accomplishes this when others react to her major: "[S]ome of the times I get like, 'Aren't engineers supposed to be boring?' Or, 'Aren't physicists supposed to be, like, scared of people?’ I'm like, 'No, I'm pretty normal. . .We're just really excited about really boring things." Not only is Stephanie aware that she does not fit the "ideal" - her comments signal that she accepts this, allowing her to act when provided opportunities to influence others' perception of the "ideal."

What is also interesting about the way Stephanie navigates her inability to fulfill the "ideal" scientist stereotype is that like Bethany, she finds herself aware that she also does not fulfill the assumed female ideals: 
I'm not the type of person who needs to plan out how my career is going say around me having kids, or getting married, or having a house. I'm not that type of person at all. I don't see it so much as such a step-by-step process. More so because that ideal scares me. Thinking about that is kind of creepy. [laughs] I think identity is not just race but is age, and experience, and background. If I'm ever at a point where I do want to get married, I do want to have kids, these type of platforms are in place, rather, I know what the plan is for this.

Stephanie operates in comfort knowing that although she does not fit the "ideal" for her physics majors, nor perhaps for her gender, these roadmaps can be helpful to her regardless. Just as sailors have utilized celestial navigation both to sail set routes and create new ones, Stephanie views notions of gender and science ideals not as binding, but rather as a guiding resource as she creates her own path: "I try to - not to combat the stereotype, just because the stereotype is so wrong that all I have to do is show up to the classroom to beat that - I try to go a bit further."

\section{Gender Roles and Goals Redefined}

In addition to their willingness to redefine the ideals of gender and STEM, a fascinating dimension of this category of experience is how much they have in common with the other category of experiences, which fulfills their communal values. Both Stephanie and Bethany are highly communal, often framing their examples as participants in the communal category in terms that highlight connecting with others, working with others, and helping others. The ambassadors are also both highly reflective and aware of gender in STEM expressing a similar level of thoughtfulness as those in the reflective category of experience about experiencing visible and invisible forms of discrimination. However, whereas we saw some of the ways the incongruent identities of the communal and reflective participants impacted their ability to reconcile their 
communal roles in the present and their science goals in the future, Stephanie's examples often epitomize the way her congruent identity and communal values guide her interactions with her peers. During a class project when she was working with three other male peers, she said one of them took the lead, would not accept her suggestions and ideas, and suggested to her, "Why don't you go start working on the PowerPoint." Stephanie said:

I did eventually step up and say, "Hey, why don't we try this?" And they weren't understanding how I was explaining it. I was like, "Alright, well then let's get up and actually go find something that looks like it." I took us out of that area where our conversation was happening and we walked around and found something and physically put it together. In that type of dynamic, I think the other two guys was like, "Oh, I now understand what you're trying to do. This makes way more sense. Let's do it like how she's doing it."...We ended up going with my idea.

As Stephanie reflected further on the continued resistance of her peer who had originally taken charge even after the other group members agreed to pursue her approach to the project, Stephanie said she was patient, yet firm with him because: "We're all Physicists. We all know what we're doing. We all have different strengths. Yes I can make things look gorgeous but I also can do the calculations." In this way, Stephanie's confidence in her own physics identity, as well as her values for team work and connecting with others allowed her to take the lead while showing her teammates respect.

Although Stephanie and Bethany express more in common with the communal category of experience than with the solitary category of, the way they articulate their independence, individuality, and their reflections on some of their isolating experiences in their majors indicates shared experiences with that category as well. 
The ambassadors spoke of roles and goals both now, and in the future, that they hope to hold that will afford them the ability to pursue their science interests while also contributing to their fields in communal ways. Bethany and Stephanie are performing a form of "cognitive flexibility" according to Diekman et al. (2017) by building roles for themselves into their future goals that will meet their communal needs, particularly if their science goals may not afford these communal goals in their work spaces. In this way, Bethany and Stephanie have identified ways to create congruence for themselves in spaces where they perceive these goals and accompanying roles to not be readily available.

It is unclear if Stephanie and Bethany's physics identities also include an interest in serving as ambassadors for the field because they can personally relate with many different types of people, or if because they relate with many different types of people on a personal level because they are able to adjust their communication style in real time making them attracted to the role of ambassador. Regardless, both Stephanie and Bethany share commonalities across all of the categories of experience, which may aid them in their goals to recruit, mentor, and retain women and other minorities in physics.

\section{Outcome Space Discussion}

Marton and Booth (1997) elaborate on three distinct criteria to evaluate a phenomenographic outcome space: 1) distinctive categories presenting unique relationships with the phenomena as experienced by the participants; 2) these distinct categories are presented as they logically relate to each other, "frequently hierarchical;" and, 3) the fewest number of categories possible are used to represent the data (p. 125). These criteria will be utilized to discuss the quality of the outcome space presented here. 
First, the different relationships participants experience with the conceptual framework were used to clearly delineate the distinctions between the categories. For example, the assured, the solitary, and the ambassadors experience high levels of congruence between their social roles and their science goals. However, the relationship each of these groups experience with congruence differs. The well-developed physics identities of the assured allow them to both anticipate and experience internal and external barriers with confidence and perseverance. The low communal goals and welldeveloped intrinsic agency of the solitary supports their preferences to work alone, thereby fulfilling stereotypes about the ideal scientist congruently. And the ambassadors create their own relationships with congruence as they redefine the ideal through themselves and prioritize the bird's eye view of their field and helping others over their individuality thus fulfilling communal goals.

The communal and the reflective experience relationships with incongruence, marked by unique differences across the two categories. For example, the communal want to connect with others, attend to others, and help others tangibly. The communal take an "all or nothing" approach to this incongruence thereby impacting their goal affordances in distinct ways, either by choosing a physics program with a highly communal atmosphere, choosing an application of physics that allows for more tangible ways to help, or by exiting the field to take up an endeavor that does afford higher levels of communality. The reflective had the most complex relationship with incongruity, marked by a heightened awareness of the ideal assumptions the gendered roles. Lower competence and performance beliefs contribute to the differences between the reflective and the ambassadors; their lack of belief in themselves and their ability to cope results in 
the reflective having trouble resolving scenarios, unlike the ambassadors who give themselves a break.

The second criteria Marton and Booth (1997) suggest in determining the quality of the outcome space is that the presentation of the categories follows a logical order organized hierarchically by complexity and inclusivity. Ordering the categories of experience that make up this outcome space by the assured, the solitary, the communal, the reflective, and the ambassadors accomplishes this. This is because the assured and the solitary experience relatively straightforward, congruent relationships with the physics identity, gender role and goal congruity, and assumptions about the ideal. Although in different ways, participants in both of these groups hold congruent beliefs about these concepts, and practice congruent behaviors. For example, the way the assured experience a set path for themselves towards their short term and long term goals is by believing that with hard work, dedication, and the right environment, they will be able to achieve their goals in physics and astrophysics. And the solitary are fulfilling some of the assumptions about the ideal scientist in that they prefer to work alone and rely on their own independence to achieve their goals.

The communal and the reflective are presented next because these two groups experience incongruent relationships between their social role affordances and their goal affordances. The communal are presented first because their relationship with this incongruence is directly influencing the way they anticipate these affordances and adjust their future goals in order to afford increased congruence. The reflective category was presented after the communal group because the relationship participants in this group have with the conceptual framework is more complex, and participants in this group did 
not articulate a change in goals or behaviors as influenced by the incongruence they experience. Rather, they articulated searching for examples of how they might find congruence in the future either through their science community, or through their roles as ambassadors in the future. However, at this time, the reflective group have not made choices or enacted decisions that resolve this incongruence.

The ambassador category was presented last because from a hierarchical standpoint, this group is the most inclusive of the other categories and experiences the most complex relationship with congruence. For example, participants in the ambassador category resolve the incongruence felt by the reflective by envisioning and carving out definitive new ideals, and by seeking new roles to be taken on in traditional physical science settings.

The five categories of experience are presented in hierarchically structured terms of increasing complexity and inclusivity in the relationships participants experience with the conceptual framework, as recommended by Marton and Booth (1997). In other words, the fact that the assured is delineated first, for example, or that the ambassadors are presented last, should not signify any rank or privilege to participants who experience these types of relationships with the conceptual framework. Rather, these categories are presented in this manner because they are marked by sub-outcomes representing relationships with the conceptual framework that range from relatively straightforward to complex and inclusive of outcomes from other categories.

Finally, the five categories delineated above have been determined to be the fewest number of categories to adequately present all of the outcomes and sub-outcomes within this outcome space, as per Marton and Booth's (1997) recommendations. An 
example of how this decision was made can be best described through the analysis and presentation of the ambassador category because this example holds the most robust borderline cases from other categories, as well as opportunities to place participants from this category in other categories.

Borderline cases were not many, but those in question caused a number of conversations and multiple cycles of analysis to ensure that they were placed in the category that most appropriately captures their relationship with the experienced phenomenon at the time of the interview. Stella, for example, was considered a borderline case between the assured and the communal categories; the way she masked much of her high competency and performance beliefs within communal language meant to create connection with others made it challenging to place her. After conducting the interview, I had made field notes stating how impressed I was by her confidence and the clarity with which she described her abilities and interests. However, when I read through the transcript the first couple of times I was struck not by the confidence I remembered from the interview, but rather noticed how imbedded this confidence was in language I deemed at the time as symptoms of "imposter syndrome." She studies in a group setting often, and shared no examples that placed her in the solitary or the reflective categories; to break this tie and place the data into the appropriate category, Akerlind's (2012) recommendation that the "underlying meaning of virtually the whole transcript" became critical. The underlying meaning of Stella's transcript was marked by confidence, grit, and a sense of assurance despite some of the flowery language she uses to convey her assurance. 
Carly served as another important borderline case. Carly mentioned an interest in ambassador-like involvement in the future, and she had already taken on some of those roles in her academic program like the TA position she held during some of the examples shared in the reflective group. However, the reason she was placed in the reflective category rather than the ambassador category was because of the heightened, and unresolved nature of her awareness of gender roles and goals in physics. This underlying meaning was determined as more important than the singular quote in which she expressed interest in serving as an ambassador to women and other minorities in physics in the future. It is possible that in the future she will take up this role, but at the time of the interview and the context within which she framed her experiences, it was determined that her placement in the reflective group was more appropriate.

Carly's borderline status in particular caused dialogue about whether or not participants could, or more importantly, should be placed in multiple categories for the sake of this outcome space. The decision to keep participants in one category of experience only was intentional in order to follow the "parsimoniousness" in the number of categories, and to define the categories with as much clarity as possible.

The two ambassadors also served as potential borderline cases because both Bethany and Stephanie shared many common experiences and relationships with experiences across the other four categories. I could have easily placed Stephanie in the assured or reflective categories, and Bethany could have fit in the communal or reflective categories exceptionally. It was intentionally determined to highlight this unique aspect of their experiences, coupled with their express goals to aid their fields in recruitment and retention efforts as a separate category that was inclusive of aspects of the other 
categories, rather than place these individuals in other categories and present only four within the outcome space.

These examples of the nuances found within the data and the decisions made throughout the analysis provide context for the richness in the descriptions of the five categories of experience making up this phenomenographic outcome space. Therefore this outcome space meets the criteria provided by Marton and Booth (1997) as well as Akerlind (2012).

\section{Summary}

This chapter delineated the five phenomenographic categories of experience used to describe the qualitatively different relationships the 18 participants in this strand of the study have with the conceptual framework which were: (a) The Assured, (b) The Solitary, (c) The Communal, (d) The Reflective, and (e) The Ambassadors. These categories of experience provide the rich detail to answer the qualitative research questions which will be addressed in the next chapter in greater detail, followed by a presentation of the explanatory sequential mixed methods data analysis and interpretations and discussion. 


\section{CHAPTER VI}

\section{DISCUSSION}

This chapter includes a summary of the study followed by an overview of the answers to the quantitative and qualitative research questions and a presentation of the explanatory sequential mixed methods findings and interpretations. A look at the limitations of this study, the implications for theory, research, and practice, and the recommendations for future research will also be presented. This chapter will close by discussing the conclusions of the study and a final summary.

\section{Summary of the Study}

Persistent gender disparity in some STEM fields indicates that advancement in those fields is limited to a fraction of available contributors. Increased research and discussion focused on the reasons why girls and women choose male-dominated STEM fields such as physics, engineering, and computer science less than their White and Asian male peers, include the ways this phenomenon influences, and is influenced by, higher education. Focusing research on this phenomenon contextualized in higher education settings provides an important perspective on a particular moment in a student's life when they experience the culture of these communities, as well as the recruitment and retention efforts of community members. In other words, the recruitment and retention efforts for these fields are both formal and informal, and take place over the course of a student's childhood, and arguably her lifetime, with higher education serving as an influential time point along this pathway.

According to the National Science Foundation (NSF) and American Physical Society (APS) IPEDS Completion Survey data, women earned approximately $20 \%$ of the 
approximately 8,000 bachelor's degrees awarded in physics in 2015 (NSF, 2015). This explanatory sequential mixed methods study utilized a national data set of participants who completed the pre-conference registration survey for the 2016 Conferences for Undergraduate Women in Physics (CUWiP) for both the quantitative and qualitative research phases. The CUWiP data used for this study $(n=900)$ therefore represents a sizeable portion of women engaged in physics majors in the U.S. overall.

This study was designed to examine the way women who have chosen to major in these male-dominated fields of study experience this pathway. The theories and concepts chosen as the framework guiding this study consisted of: physics identity, (in)congruent gender roles and goals, perceptions and assumptions about the "ideal" scientist, and how participants use (or do not use) this information to inform their decisions to persist in their major.

\section{Findings and Interpretations}

Chapter 4 presented the findings from the first phase of this study: A series of statistical analyses of the 2016 Conferences for Undergraduate Women in Physics (CUWiP) pre-conference registration survey data collected by APS. Chapter 5 presented a rich description of the second, qualitative phase of the study. The qualitative data was collected through 18 phone and Skype interviews with participants from the 2016 CUWiP data set, resulting in five phenomenographic categories of experience: The Assured; The Solitary; The Communal; The Reflective; and The Ambassadors.

\section{Response to Research Questions}

Due to the explanatory sequential mixed methods design of this study described above, the first three research questions were designed to be addressed by the quantitative 
phase of the study, and the final two questions were designed to be addressed by the qualitative phase of the study. The statistical analyses presented in Chapter 4 and the categories of experience presented in the phenomenographic outcome space of Chapter 5 helped to answer these five research questions discussed in the following section.

\section{Research Question 1}

The first question focused on the quantitative data was: Is the way undergraduate women in physics see the "ideal" physics student related to their physics identity? A linear regression was utilized to answer this research question, finding a significant correlation at the $p<0.001$ level exists.

\section{Research Question 2}

The second research question focused on the quantitative data was: How does gender role congruity relate to physics identity? To adequately answer this research question, a series of tests were run on the data to determine the relationships between physics identity and gender goals overall, as well as the relationships between physics identity and the three individual levels of the gender goals: communal, extrinsic agency, and intrinsic agency.

The linear regressions run on the variables separately (e.g., physics identitycommunal goals; physics identity-extrinsic agentic goals; physics identity-intrinsic agentic goals) yielded significant correlations at the $p<0.001$ level for each of the three gender goals. The relationship between intrinsic agentic goals and identity had the largest effect.

The multiple linear regression run with all three gender goals on identity revealed that intrinsic agentic goals showed the most significant relationship with physics identity 
at the $p<0.001$ level. This test also revealed a smaller but significant correlation between communal goals and physics identity at the $p<0.01$ level. This test revealed no significant correlation between extrinsic agentic goals and a physics identity for participants in this study. In other words, with intrinsic agentic and communal goals in the model, extrinsic agentic goals were no longer significantly correlated with physics identity.

Examining these relationships between gender roles and identity individually and in combination allows us to see the significance of the relationships each of these gender roles has with physics identity for undergraduate women majoring in physics, but highlights the particularly critical nature of intrinsic agency for these women, and the relatively insignificant relationship between extrinsic agency and identity when tested in combination with the other two dimensions of gender roles.

\section{Research Question 3}

The third and final quantitative research question focused on the quantitative data was: What factors (i.e., physics identity, "ideal" scientist, gender role congruity) relate to plans to persist? A series of linear regressions were run to address this research question. The findings reveal that physics identity is the most significant indicator of self-reported plans to persist to bachelor's degree graduation for female physics majors $(p<0.001)$. The results also indicated a significant negative correlation of communal goals with bachelor's degree persistence at the $p<0.01$ level. The intrinsic agentic goals, extrinsic agentic goals, and perceptions of the "ideal" scientist variables had no significant correlation with self-reported plans to persist to bachelor's degree graduation. 
The same series of tests were run on other levels of self-reported persistence plans such as graduate school and career plans. In addition to a significant correlation between identity and bachelor's degree plans, a significant correlation between identity and both graduate school and career plans was discovered at the $p<0.001$ level. Intrinsic agentic goals were significantly correlated with graduate school and career plans at the $p<0.05$ level. The remainder of the variables, communal goals, extrinsic agentic goals, and perceptions of the "ideal" scientist were not correlated with the graduate school and career persistence plans.

These findings are particularly interesting because of the way they clarify and expand on Diekman et al.'s findings (2010). For example, Diekman et al. framed communal-goal endorsement as negatively predicting STEM interest defined as persistence, confirmed in part by these findings. However, communal-goal endorsement was found to be positively correlated with interest as a dimension of physics identity rather than a dimension of persistence. This is meaningful because physics identity was in turn found to be the strongest predictor of all levels of self-reported persistence plans (i.e., bachelor's degree completion, graduate school, physical science-related career plans). This nuance is important because while Diekman et al. found that communal-goal endorsement negatively predicts STEM persistence plans as did this study, this study found that intrinsic agentic goals positively predict physics identity the most, confirming Hazari et al.'s (2010) findings. Communal goals positively predict physics identity, and extrinsic agentic goals do not predict physics identity. Regardless of these endorsements, physics identity was found to be the strongest predictor of persistence within the conceptual framework, and communal goal-endorsement was found to negatively predict 
bachelor's degree completion and have no correlation to graduate school plans and career plans. This level of nuance in fact supports Diekman et al.'s (2017) call to increase research on the ways STEM fields afford communal-goal outcomes and anticipated incongruities, rather than focusing on the ways in which women and other minorities can align more with science experience and self-efficacy.

\section{Research Questions 4 and 5}

The fourth research question focused on the qualitative data: How do the experiences of undergraduate women in physics majors delineate their perspectives related to their physics identity, the "ideal" physics identity, and gender role congruity within their chosen field of study? As discussed at length in Chapter 5, the data analysis determined that participants in this phase of the study experience the concepts under investigation in qualitatively different ways. These qualitative differences were organized by experiences and the underlying meaning of the transcripts, then by the relationships participants have with these experiences, and then finally by participants who shared similar relationships with their experiences of the conceptual framework. Some of the qualitative participants had well-developed physics identities, while some experienced lower performance and competence beliefs, and recognition that was perceived as absent or negative. While most also articulated versions of an "ideal," for some this ideal posed a threat to their identity and for others it did not. Finally, there were a spectrum of roles and goals related to gender articulated by participants, with intrinsic agency and communal dimensions playing more prominent roles across the sample than extrinsic agency. The resulting delineation of the qualitatively different 
ways participants experienced relationships with the conceptual framework answers the next, and final research question.

The fifth research question focused on the qualitative data was: How are the experiences of the participants similar, or different, based on the theoretical framework that guided this study? The qualitative data analysis yielded five distinct categories of experience presented in Chapter 5. These five categories of experience delineated the similarities and differences in relationships participants experience with the conceptual framework in their physics majors. These five categories of experience are: The Assured; The Solitary; The Communal; The Reflective; and, The Ambassadors.

The assured experience a relatively congruent relationship with gender roles and goals through well-developed intrinsic agency and an appreciation for communal spaces within a stereotypically non-communal major, such as Samantha's positive experience as a physics major at a women's college. The assured also experience congruence in their assumptions about the "ideal" even considering themselves the "ideal" at times such as Hilary and Madelyn's experiences. And finally, the assured experience well-developed physics identities, even when masked by humility in order to connect as in Stella's case. The physics identities of the assured are marked by early interest, high competency and performance beliefs, and many varied examples of recognition. One of the important features of the assured is that they find congruence with these identities and roles through status quo acceptance, as compared with the ambassadors who create their own congruence by redefining accepted norms and assumptions.

The solitary experience high levels of well-developed intrinsic agency such as individualism, self-direction, and independence; this group also values intrinsic agentic 
goals focused on having solitary work environments, as described by all five participants in this group. Working with others and related communal dimensions are valued when it benefits them individually, such as Gloria's and Matilda's examples. This solitary positioning can lead to absent, limited, or self-identified forms of recognition which can further isolate participants in this category and have a negative influence on their competency and performance beliefs. The solitary positioning and individualistic values lead to small networks and creates a limited frame of reference for physics identity. Although qualitative work is not representative, it is interesting to note that this group shares the most conforming position with stereotypic physics roles and emerged as the largest category of experience with five participants.

Participants in the communal category of experience prioritize connecting with others, helping others, and attending to others over their own needs in group work and leadership experiences. This resulted in participants experiencing incongruent relationships with identity, "ideal" assumptions, and particularly with gender roles and goals in physics since communal roles were not always readily available. Thus, each of the three participants in this group sought goals that they anticipate will afford them increased congruence with having communal roles in the future, such as Naomi's desire to earn an MBA and open a homeless shelter, and Gabriella's goals to pursue medical physics so that she can help people tangibly. Marion's willingness to pursue a graduate degree in physics stemmed directly from her knowledge of the highly supportive and communal culture of that specific academic program. Each participant in this group sought to reconcile the incongruence they were experiencing as communal-oriented physics majors. 
The incongruent relationships with the conceptual framework experienced by the reflective is marked by a heightened awareness about gendered roles and goals, as well as assumptions about the "ideal" and their inability to fulfill these assumptions, roles, and goals in the traditional sense. Physics identities are disrupted at times for participants in the reflective category as a direct result of their heightened awareness of negative recognition. This heightened awareness is further compounded by their deprioritized sense of extrinsic agency leading participants in this group to cede power to those they perceive to have the authority to resolve, or leave unresolved, the injustices they observe or experience. Additionally, the heightened awareness of behaviors, language, and roles that perpetuate inequalities also allows participants in this group to seek and appreciate positive experiences and relationships related to the conceptual framework.

The ambassadors were presented as the final category of experience as the two participants in this category experience complex, sometimes reconstructed, congruent relationships with the conceptual framework inclusive of aspects of the previously delineated categories. Both Bethany and Stephanie could have been placed in other categories, but articulated behaviors and values throughout their interviews that set them apart from the remainder of the participants; for example, their willingness to question the goals and roles surrounding gender, race, and the "ideal," the way the reflective do, but articulating the way they move past these concerns quickly the way the assured do, or address them directly in ways other participants were not able to articulate. Both Bethany and Stephanie articulated clear goals to incorporate mentoring and ambassadorship for women and girls in STEM now and throughout their careers. 
These categories of experience represent significant variation in the way women majoring in physics experience the phenomenon of being a female in a male-dominated academic major. Based on the statistics available about the number of women participating in physical science majors across the U.S. and the examples shared by participants in this study, these women are often one of a small handful of females in their program, and often report an equally small number of female professors teaching in their academic programs. Therefore, they may not have the opportunity to be exposed to this diversity in experiences seen within the qualitative data, which could influence the way their relationship with these experiences develops throughout their bachelor's degree and beyond.

As discussed in Chapter 5, these categories were presented in this order due to the hierarchical order ranging from relatively straightforward, congruent experienced relationships with the conceptual framework (i.e., assured and solitary), to incongruent and more complex relationships with the conceptual framework (i.e., communal and reflective), and finally, the most inclusive and complex relationships with the conceptual framework (i.e. ambassador). The categories of experience feature the ways participants experience similar relationships with the conceptual framework, as well as explore the depths of the differences across the relationships with these experiences. This presentation of the data serves as an organizing mechanism to explore the qualitatively different ways participants in this phase of the study experience the conceptual framework used to examine the phenomenon of being a female in male-dominated undergraduate major that has socially prescribed roles and goals such as physics. Additionally, this approach has contributed to the dialogue advanced by Diekman et al.'s 
(2017) findings by furthering the integrated examination of the agentic and communal orientations of successful STEM roles and goals.

\section{Explanatory Sequential Mixed Methods Analysis and Interpretation}

It is important to integrate the discussion of the quantitative and qualitative data analysis and results for the purposes of addressing the explanatory sequential mixed methods design of this study (Creswell, 2015; Morse \& Niehaus, 2009). Drawing inferences about how the qualitative results help to explain the quantitative results served as the cornerstone for the development and execution of the qualitative phase of the study including: protocol development, the participant recruitment process, and the structure of the outcome space delineating the qualitatively different ways participants experience the conceptual framework. Following the qualitative data analysis and outcome space delineation, this information can be used to infer and understand additional nuances discovered by this study.

\section{Research Question 1}

Seeing that a significant relationship $(p<0.001)$ exists between the participants' perceptions of the "ideal" physics student and their physics identity alone cannot tell us much. However, examining the qualitatively different ways participants in the second strand of this study experience this relationship does. Participants in some categories of experience accept the assumptions about the "ideal" and believe they fulfill such assumptions (e.g. the assured), they experience heightened awareness related to these assumptions (e.g. the reflective), or even actively seek to redefine these assumptions for themselves and others (e.g. the ambassador). And as Gabriella astutely observed: 
The funny thing is, the guys who were like that [i.e., brilliant in a way that they didn't have to study and could still do well on the exams] aren't going on to do anything with it, yet at least. Maybe they will later. But, the kind of students who work really hard at it, all of us are going to grad school. I think the kind of person who is naturally good at it doesn't learn how to work at it and maybe doesn't have that ethic that they need to go on in it, which is so weird.

Like Gabriella, participants across the five categories of experience their own version of this knowledge that hard work is an important component for successful physics majors. Although participants in this study articulated their value of the role hard work plays in their academics, each of the categories experience this perceived incongruence with stereotypic "ideal" assumptions in different ways. Considering the findings of Bian et al. (2017) and Carlone (2004), that girls are taught that working hard is an important component of being a "good girl" in school, and that they are aware of this as early as 6 years old, it is interesting to see how the participants in this phase of the study either accept the "ideal," live in disrupted states, or reject and redefine these assumptions.

For example, participants in the assured group reported little difference between their descriptions of the "ideal" and their own physics identity. Hilary's affirmative answer to the question, do you consider yourself to be the best student in your class, exemplifies the way some who experience an assured relationship with the conceptual framework guiding this study see themselves as the "ideal." Hilary has even expanded her own definition of the "ideal" beyond the assumption that you, "have to be a genius" to include behaviors such as work ethic and network building, privileges such as attending school on a scholarship that allows her to focus solely on her studies and research, and incorporating grit into your mindset. 
A thorough examination of the reflective category of experience shows that these participants experience a different yet meaningful relationship with the "ideal" and their identity. For example, Dahlia's reflection about the way comments from others related to how smart she must be to be a physics major used to make her feel "a little bit special," or even "better" than others. However, she says that she knows this perspective can be problematic for herself and others, and therefore she has made a conscious effort to not think or behave in ways that perpetuate the stereotype that success in her major is inherent. Dahlia's heightened level of awareness and reflection about the "ideal" assumptions and expectations of those in her major result in heightened incongruence at times, signaling the way her awareness of these issues leads to incompatibility with her physics identity.

Another example is made clear by participants in the ambassador category of experience. These participants have complex relationships with the way they perceive the "ideal" assumptions in science, and therefore they are forging their own paths and redefining the "ideal" for themselves and others. Bethany's experience of being embarrassed to disclose her major when performing highly female roles, such as getting a manicure, is confirming Gonsalves' (2014) findings that the culture of physics positions the "girly girl" as contradictory to the "ideal." The way Bethany reconciles this contradiction is by making conscious choices about when to disclose her major, and to serve as a role model for other women in physics. In another example, Stephanie's concise descriptions of the way she defies stereotypes about the "ideal" in her physics and mechanical engineering majors, which she says are "so wrong" that she redefines these assumptions easily: "all I have to do is show up." But is quick to add that for her 
this is not enough, she tries "to go a bit further," and tries "to remind myself that I am dealing with a lot more than what that guy over there is dealing with," so she fortifies the competence and performance dimensions of her physics identity.

Looking at these three examples of the qualitatively different ways participants in the second strand of this study experience the significant correlation between the "ideal" and their physics identity provides nuance and context not readily available in the statistical outcome alone. Rather, the qualitative data is important to the interpretation and discussion of the significance of this relationship.

\section{Research Question 2}

The qualitative findings further explain the findings related to the second research question in a number of ways. To discuss the ways Diekman et al.'s gender role congruity model relates to physics identity, it is important to consider the qualitatively different ways participants in the second strand of the study experience these two theories of the conceptual framework. This discussion will explore the relationships between gender role congruity theory and physics identity theory in the order of their significance: intrinsic agentic, communal, and extrinsic agentic.

First, the most significant correlation found between intrinsic agency and physics identity confirms the findings of Hazari et al. (2010): “[T]he strongest predictor of physics identity is the desire to pursue a career that would result in intrinsic fulfillment through working with knowledge, skills, or products." Participants in the qualitative strand of the study confirmed this, first through their responses on the CUWiP survey, then later in their examples shared during the interviews. Only two qualitative phase participants had scored low on the intrinsic agentic goals measure and after analysis of 
the qualitative data, both of those participants were placed in the communal category of experience. Gabriella for example, who had graduated with her bachelor's degree in physics at the time of the interview, was pursuing a graduate degree in medical physics to, "help people tangibly" therefore pursuing communal goals rather than intrinsic agentic goals.

On the other hand, participants in the remaining categories of experience scored mid-level through very high on the intrinsic agentic goals measure of the CUWiP survey, which was confirmed through the presentation of the qualitative outcome space. Among the strongest examples of this relationship between intrinsic agency and physics identity is seen in Brooklyn's summary of her goals: "What I am super interested in is just diving into research. Even if it's stuff in a small basement room in a cold building by myself for hours, and hours, and hours." The way Brooklyn explicitly ties the interest dimension of her physics identity with her goal to conduct research, including the prospect of conducting this research independently, is seen as a viable and fulfilling future.

The qualitative findings further expand our understanding of the relationship between intrinsic agency and identity in that many participants in the second phase of the study, and especially those in the solitary and assured categories of experience, work well independently and value individualism and self-direction. Participants in the solitary group value communality only as far as they see the benefits for themselves in reaching their individual goals. Finally, many of their intrinsic agentic examples were framed by identity dimensions such as interest as well as the motivation to seek competence and performance through demonstrating skill. 
A smaller yet still significant correlation between communal goals and physics identity was found at the $p<0.01$ level. This is not surprising; in fact, this finding confirms Diekman et al.'s (2017) findings that the degree to which women endorse communal goals is high, even within STEM. The CUWiP data compares women to other women, and women who are communal have slightly higher physics identity scores overall (Hazari et al., 2010). This appears counterintuitive until we consider the recognition component of identity. To develop a physics identity, female students need to feel recognized which requires greater and meaningful interactions with others related to content. Non-communal students, such as the solitary, will be less likely to engage in these interactions. Communal students will therefore engage in the community, feel recognized as a part of the community, and feel recognized through this acceptance.

The qualitative data further explains this finding in a number of meaningful ways, including the criteria utilized to determine the organization of the participants in the categories of experience. For example: The Ambassadors reconcile the incongruence by seeking communal roles integrated into their career plans that include recruitment and mentoring roles for other women and girls interested in physics. The assured and the solitary participants do participate in communal roles, even valuing these roles highly, but this is often motivated by the identity benefits such as developing competence and increasing performance. The communal, the reflective, and the ambassadors articulated varied relationships existing between their communal roles and goals and their physics identity.

For example, from the communal category, Marion's description of the culture of her physics program defies some of the stereotypes confirmed by earlier findings: 
I do get the impression that [outsiders perceive physics majors to be] somewhat isolated and ... Not introverted, but you tend to work by yourself, and it's all theory and formulas and there's no real collaboration. But in reality it is almost 100 percent collaboration.

Despite how different this perspective on physics as affording this level of communalgoal achievement, Marion's perspective ultimately provides her with a sense of anticipated and experienced communal-goal congruence, further integrating the way her communal goals are supported by her environment, which in turn supports her physics identity, "it's just a terrific network of people and a fun curriculum." Throughout Marion's examples, communality and physics identity are intertwined in this way. For Marion, the way her program defies some of the stereotypes about physics culture has facilitated the congruence she experiences between her communal-goal endorsements and her physics identity development. This manifests as a form of community acceptance and recognition for Marion that allows her to make plans to pursue a physicist career path despite her highly communal orientation.

In another helpful example with regards to the way qualitative participants navigate the relationship between communal goals and physics identity, Carly, a member of the reflective category of experience articulated the nuanced differences when working in a female-dominated group as opposed to working in a male-dominated group:

[I]t's really interesting because when there are more females in the group, I don't know if they just noticed that there's more females in the group, or it is just the females see that there are other females in the group and they make a serious effort to try and acknowledge everyone's ideas ... which we might end up talking for a little bit longer on our game plan as to how should we do this. But, we usually get the job done, we get it done just as quickly. 
Carly's performance and competence beliefs are supported by her communal-goal endorsement, rather than contradicted. She compared this congruent experience with incongruent experiences she has had in male-dominated work groups, in which male peers do not make eye contact with her while she speaks but make eye contact with each other, or they interrupt her when she is speaking. Carly perceives these behaviors by her male peers to be antithetical to her communal goals, requiring a resolution on her part:

And there was a group where there were three men and me and one other woman and the same thing was happening so she and I broke off and created our own group so.

Because she does not experience diminished competence or performance outcomes when working in a female-only work group, Carly anticipates separating herself and her female teammate from the males will resolve her experienced incongruence and realign her communal goals and her physics identity.

In the final example of the way the qualitative findings further explain the relationship between communal goals and physics identity, the way a member of the ambassador category, Bethany, anticipates communal-goal incongruity serves as an exemplar:

That's like another question that I'm thinking about because I do want to help people in my life and I know studying like an exoplanet isn't really going to, or studying the history of the universe isn't going to directly help humankind, at least right now. But, I do want to, I do really enjoy studying that, I think it's interesting. It's something that I'm still thinking about.

Despite this anticipated gender incongruity, her physics identity continues to motivate her to persist. One of the ways that Bethany reconciles this perceived incongruent goal affordance is to incorporate volunteer work to mentor, recruit, and retain young women 
and girls in STEM into her goals: "I'd like to do some sort of outreach project like that throughout my life." Her willingness to make concrete plans to proactively reconcile her physics identity with her communal goals suggests that she believes that pursuing a physical science career may or may not directly afford her communal activities, but because she knows she needs this, she will seek opportunities to incorporate this into her volunteer involvement in the STEM community rather than seeking ways to reconcile this by pursuing a different field of study or work.

Finally, the way the qualitative findings further explain the quantitative finding in response to the second research question, that extrinsic agentic goals do not correlate to physics identity, it is important to look at the way many of the qualitative participants described muddled, one-off, or nonexistent experiences with extrinsic agency. Participants in the reflective category of experience exemplified a muddled relationship with extrinsic agency with the most clarity. In a number of examples shared in Chapter 5 we saw the reflective react to their observations of gender-motivated bias with a desire to seek resolutions, however they decided to cede this power to others they perceived to have the authority to enact resolutions rather than to exercise this type of agency themselves. In other words, the reflective see roles and goals being played out that they perceive to be not only incongruent but also unjust, and yet they lack extrinsic agentic awareness and skills that afford them opportunities to influence a different outcome - an outcome they see as more gender-equal and therefore more just - themselves. The way the reflective use what extrinsic agency they can access to cede power to someone else they believe will be able to influence the injustice ultimately leaves successful resolution, or lack of resolution, in another's power resulting in missed opportunities to exercise 
their own agency. Beyond the reflective, extrinsic agency indicators related to physics identity did not show up in the qualitative data in meaningful ways beyond the occasional one-off comments (e.g., making money, status and power in their careers, etc.).

These various examples of the ways the qualitative findings further explain the quantitative findings related to physics identity and gender role congruity theory provide an overview of the complex relationships participants navigate when integrating identity and gender. The presentation of the qualitative findings in distinct categories of experience contextualized these relationships further, allowing for the depths in differences to be illuminated. In the following section, the ways in which the qualitative findings further explain the relationships between the conceptual framework and selfreported plans to persist at the bachelor's completion, graduate school, and physical science career levels will be explored.

\section{Research Question 3}

In review, the third research question asked: What factors (i.e., physics identity, gender role congruity) relate to plans to persist (as measured by bachelor's degree completion, graduate school plans, and physics-related career plans)? The ways in which the qualitative findings further explain these quantitative findings provide additional depth to this discussion and create opportunities to consider how these findings contribute to the research on increasing participation of women in STEM. Perceptions about the “ideal" science student were not correlated with any level of persistence, nor were extrinsic agentic goals, despite agentic goals being found to be important predictors of persistence by Diekman et al. (2010). This is likely due to the fact that this study split agentic goals into extrinsic and intrinsic dimensions, and intrinsic agentic goals were 
found to be predictive of graduate school and career plans, but not bachelor's degree completion, a nuance that will be explored in this section. In addition to this discussion about intrinsic agentic goals and persistence plans, the ways communal goals negatively predict bachelor's degree completion and do not predict persistence beyond graduation will be examined. Finally, this section will explore how the qualitative data further explains physics identity as the strongest indicator of persistence at all levels. This is the final mixed methods discussion presented in this study.

Intrinsic agentic goals were not identified as predictive of bachelor's degree completion, however a significant correlation was found at the $p<0.05$ level for intrinsic agentic goals and persistence plans measured by graduate school plans and physicsrelated career plans. Again, Brooklyn's description of her goals to do research even if she's isolated "in a small basement" for long periods of time epitomizes the way some participants in the qualitative phase of the study anticipate their career goals will align with their intrinsic agentic goals revolving around individualism, independence, and selfdirection. Dakota's articulation of this correlation is problematized by her awareness that inherent in her highly independent work style and program culture is a sense of competition that can further isolate, rather than motivate:

There's a feeling of...competition to compete with fellow classmates, and I think it probably comes from that encouragement to do solitary work. That pressure to do well on your own...It's something that I'm conscious of, and try...not to do, and just focus on myself and think about my own grades, and focus on learning the material for myself. Or, focus on the future and think that as long as I get the grades, I'll learn that in grad school.

Dakota makes that important leap at the end of the quote tying her current intrinsic agentic roles and experiences with her future goals to pursue graduate school. Diekman 
et al. (2017) use a general model of goal congruity to describe the three phase process Dakota is experiencing here. First, in anticipation of goal congruence or incongruence, in her case congruence (i.e., I work well alone and my program expects us to conduct solitary work), she feels a sense of belonging in the program.

"[T] he strongest predictor of physics identity is the desire to pursue a career that would result in intrinsic fulfillment through working with knowledge, skills, and products" (Hazari et al., 2010, p. 994). This correlation between intrinsic agentic goals and long term education and career goals again confirms the Hazari et al. findings, as well as the findings of Diekman et al. (2010), in that those with anticipated individualistic roles and goals are more likely to pursue a STEM career than those with anticipated other-oriented roles and goals. Dakota's example provides additional context for the goal-setting and decision-making processes a student constantly experiences as they decide whether or not to continue to include physics in their long-term future goals.

Shifting focus, other-oriented, communal roles and goals were not correlated with long-term plans, and were negatively correlated with physics bachelor's degree completion. The negative effect results after physics identity and the other goal congruity measures were in the model. Given the same physics identity and other goals, if you have two women with the same physics identity in a physics bachelor's degree program, the one who is more communal will be less likely to complete the degree. This confirms Diekman et al. (2010) findings that "communal-goal endorsement predicts STEM interest above and beyond [agentic-goal endorsement, and] self-efficacy" (p.1055), and for this reason, Diekman et al. (2017) focus on this issue in particular because "the communal 
dimension particularly deserves attention when explaining the gender gap in STEM pursuits.” (p. 152).

Additionally, Hazari et al. (2010) found: "personal time and people-related motivations are negatively related to physics identity" (p.994). However, these findings alone cannot delineate the kind of experiences female physics majors have with communal-goal endorsement and their short-term physics education plans. Instead, these findings raise more questions such as; do communal women feel like they do not fit in, or that physics will not fulfill their needs? And in turn, are they more likely to consider quitting physics? The qualitative findings of this study contextualize the ways undergraduate female physics majors experience these findings.

The communal category of experience provides considerable depth to these findings. Although all participants in this group had either completed their bachelor's degree at the time of the interview, or articulated clear plans to do so, each of the three participants in this group negotiate their gender incongruity in very different ways. Choosing participants this advanced in their bachelor's degree completion highlighted communal exemplars whose experiences still shed light on how being communal may be incongruent with a physics pathway and three different examples of how this incongruence can be reconciled. However, conducting a similar process with students earlier in their degree programs would have led to a different, but fruitful examination of how communal goals may lead to departure at the bachelor's level.

Take Naomi for example, who articulated clear goals to complete her bachelor's degree in physics, but beyond that she saw herself contributing to society in a more concrete manner by pursuing an MBA for graduate school, rather than a STEM-related 
degree, and she has goals to open a homeless shelter afterwards. Although she never shared an example of a time when she felt her physics degree was incongruent to her communal goals per se, Naomi provides evidence that she has in fact experienced Diekman et al.'s goal congruity processes here:

I was telling somebody my major, and they were like, "What're you going to do with that? The only thing I can think of you doing is teaching." And I began to think like that, "Dang, I'm getting this degree and all I can do is teach with this. I don't have that experience. All I am set up to do is teaching, and that's not what I want to do."...

So I was like, "Dang. Is my degree even worth anything? Do I have to change or anything?" But once I started doing research for myself, I see that I can do anything. I can go the math way, I can go the bio-medical science way, or I could get my MBA to be a manager ... which confirms this for me. . . If I want to teach, I can teach. I can do whatever. . . .I can do both.

As she experienced the perceived incongruity of her bachelor's degree and her communal goal-endorsement, she questioned her goals and whether or not to complete her bachelor's degree in physics. Naomi decided to stay, but the way communal goals negatively predict bachelor's degree persistence indicates that others with communal goals may not make the same decision. And as Naomi's example demonstrates, she has decided to complete her physics degree, but her long-term goals may take her in another direction.

In examples from the other two participants in the communal group who graduated with their physics bachelor's degree at the time of the interview, they had found ways to reconcile the incongruity of their gender goals and their physics goals. For Gabriella, she chose to pursue a medical physics graduate degree in order to apply her physics background in a way that helps people tangibly, and to be able "to see it 
immediately." However, exposure to medical physics at the undergraduate level may not be possible for most undergraduate students as most physics departments focus on more traditional fields such as condensed matter physics and high energy. Therefore students must seek out this exposure as Gabriella did when she shadowed someone in the medical physics field.

Marion also articulated examples that presented the process by which she reconciled the incongruity of her highly communal goals and her physics goals. Marion's communal-goal endorsement and physics identity are highly integrated which influences her persistence beyond the bachelor's degree in a meaningful way. She experiences highly communal roles and goals within her program, resulting in her decision to pursue graduate school in physics because of the high level of support she experiences in the classroom, in the lab, and through the dinners hosted by the female physics professors twice per semester. Not all physics students have opportunities to witness or experience communal research in physics during their undergraduate career. Thus, they may not realize these opportunities exist for them through a physics degree (Danielsson, 2009).

Finally, exploring the way the qualitative findings further explain physics identity as the most significant indicator of self-reported plans to persist across all levels of measurement reveals another interpretive perspective of the variation in the ways participants experience this relationship. The examples of the way the participants experience this relationship confirms many similar findings (e.g., Hazari et al., 2010; see also Carlone \& Johnson, 2007), including the important role the recognition dimension of identity plays in facilitating individuals' goals. 
One of the easiest ways to see the kind of experiences female physics majors have with physics identity as it relates to their plans to persist is when these are seamlessly integrated, as it is for those in the assured category of experience. Take for example Stella's description of her motivation to continue in her major and furthermore to make plans in astrophysics in the future:

Internally what tells me I'm making a good choice, I guess doing well in astronomy course versus the physics course that I take reinforces to me that I am doing a good job, I am understanding this. This is good, I can be successful here. Being happy and content with the material that I'm learning tells me that astrophysics is the place to be and the thing to study.

Stella's well-developed physics identity facilitates her persistence plans and goals through each of her identity dimensions including early interest, high competence and performance beliefs, and feeling accepted in the physics community as a foundational form of recognition.

Conversely, it is helpful to see how someone who has trouble articulating their physics identity may therefore experience less exposure to recognition, lower competence and performance beliefs, and therefore a lower level of physics identity development, which can influence their ability to set goals. Dahlia, a member of the reflective category of experience, had trouble recalling ever feeling recognized in her major or an experience when she felt confident in her performance beliefs. Therefore, at the time of the interview for this study, graduation with her bachelor's degree in physics placed her in a holding pattern: "The thing is, I didn't apply to grad school during my senior year, so I don't have the same closure [as peers], because I'd kind of been deciding whether I wanted to go to grad school at all." We discussed this pause in her goals further and beyond the connection between her lack of goals and her physics identity development, 
she cited doubts about her ability to be "happy" and "productive" (possibly alluding to declining interest and performance beliefs) unless she found the right program where she would "fit in." So here for Dahlia, not only is identity and persistence tied, but her communal goals are also influencing her ability to set future goals in physics. Dahlia's disrupted physics identity and disrupted communal-goal endorsement in turn, disrupt her persistence plans.

Echoing the sentiments of Hazari et al. (2010), "there is truth to the claim that the physics culture promotes "physics for the sake of physics"” (p. 994), Stephanie's identity and persistence are seamlessly integrated congruently as exemplified by her role as ambassador for her field:

I'm trying to be openly geeky about my major. I feel like as a Physicists one of the best qualities I feel like across the board that we have is that if you don't love what you're doing, get out. Anyone in a Physics class, loves that stuff. They have so much passion about it and they're so happy to be there that I want to share that with everyone else. I will talk to anyone.

Stella, Dahlia, and Stephanie, in their own way, are all able to articulate clear examples of the ways physics identity can predict plans to persist in influential ways.

\section{Mixed Methods Discussion Summary}

These findings contribute not only to this study, but also to the research discourse related to increasing the participation of underrepresented groups in physics overall. For example, in 2010, Hazari et al. stated: "Others who have additional motivations [beyond intrinsic knowledge-based motivations], like socio-economic concerns, will need to have a passion for physics above and beyond the norm in order to disregard such concerns and opt into physics" (p.994). This has never been truer based on these findings, which appropriately contextualize, complicate, and at times problematize the experience of 
being a woman majoring in physics today. Nearly all participants in the qualitative phase of this study cited passion and "grit" in some form or another as integral to their ability to persist in their major despite numerous internal and external barriers. Therefore, I would add to the example of "socio-economic concerns" to a long list of motivating factors women majoring in physics are also considering: assumptions about the "ideal" scientist and related stereotype threats; communal goal (in)congruity affordance concerns; agentic role (in)congruity and concerns; managing knowledge of, and personal experiences with sexism, genderism, and discrimination; and last, but certainly not least, navigating physics cultures that might include all of the above. However challenging it may be for students to navigate these varied and complex social roles and goals, it may be equally challenging for higher education faculty and administrators to implement interventions, curriculum, academic and career advising practices, and teaching practices that effectively recruit more women into physics or engineering majors in college, and furthermore to retain them. These implications for practice will be explored in the appropriately titled sections following the limitations of this study.

\section{Limitations}

Limitations of this study include those inherent in designing both quantitative and qualitative studies, including utilizing the phenomenographic approach, as well as those inherent in an explanatory mixed methods design. Additionally, the use of the binary gender identities of male and female is another limitation explored here.

The quantitative phase of the study utilized CUWiP data collected by APS, which is considered representative, but is not a random sample of undergraduate women in physics because the respondents are solely those who applied to attend CUWiP 2016. 
Perhaps this biases the sample because I may be missing isolated women who did not know about the conferences, or those who were not encouraged to apply. This same issue may also contribute to the reasons why all of the qualitative participants turned out to be persisters at the bachelor's level. And finally, a limitation to the quantitative phase of the study is the difficulty in measuring complex constructs such as identity and goal congruity survey items. The use of a mixed methods research design in this study mitigates this concern somewhat, but regardless it is noteworthy.

The qualitative design of the second phase of this study was bound by certain limitations, such that the number of women participating in physics programs in the U.S. is small, and the number of students typically attending each program is even smaller. Despite intentional efforts to guarantee confidentiality to the 18 qualitative participants, such as assigning a pseudonym, and masking identifying information in quotes when necessary, some participants were more guarded than others in the types of examples and details they shared. For example, a number of participants shared that they had not personally experienced discrimination, but nearly all qualitative participants knew of another female - either another student at their university or individuals they knew through CUWiP experiences - who had. It is not possible to know whether or not some of these examples reflected the participant's own experiences.

A limitation to the qualitative phase of the study was that the interviews were conducted by phone and by Skype, rather than in person. This was necessary because during that phase of the study, participants were located around the world either for work, school, or research. Nevertheless, this interview format can feel more transactional and less personal making the data collection process less fruitful (King \& Horrocks, 2010; 
Seidman, 2013; Winzenburg, 2011). With this limitation in mind, every effort was made to make the interview process feel personal (e.g., personally addressed email invitations via mail merge, scheduling interviews on days and times convenient to the participants, hand-written thank you notes mailed with Starbucks gift cards after the transcripts had been approved by the participants), and an emphasis placed on the interview style encouraging participants to feel comfortable and to build trust. Another limitation specific to the phenomenographic analysis process is that although the categories of experience were saturated through 18 interviews, there may be missing categories because as discussed above, the quantitative sample was not random, therefore women who did not know about or apply to CUWiP were not included in the qualitative sample.

The qualitative phase of the study was also limited by the selection criterion that participants be in their third, fourth, or fifth year of their undergraduate physics degree. Choosing participants this advanced in their bachelor's degree completion highlighted communal exemplars whose experiences still shed light on how being communal may be incongruent with a physics pathway and three different examples of how this incongruence is negotiated. However, conducting a similar process with students earlier in their degree programs would have possibly led to a more fruitful examination of how communal goals may lead to departure at the bachelor's level. This could have provided a more in-depth examination of the quantitative finding that communality negatively predicts bachelor's degree completion.

One final limitation to the qualitative phase of the study was the use of the $\$ 20$ Starbucks gift cards as incentive during the qualitative participant recruitment process. Although this incentive is considered within the limits of acceptable incentives for 
research participation (Dunn, 2002; Slomka, McCurdy, Ratliff, Timpson, \& Williams, 2007), it is critical to identify the use of incentives as a potential limitation. Because any form of incentive can influence participants' willingness to participate, and can also be perceived as possessing potential to influence the way they choose to participate (Dunn, 2002), identifying this limitation expressly is critical. I did receive a response from far more students interested in participating in this study than I needed, and no participant mentioned the gift card as the sole reason for participating; nevertheless, this limitation is worth noting here.

Another limitation to this study was the use of the explanatory mixed methods design and the phenomenographic method of qualitative data analysis, both of which are time-intensive methods. The data collection and analysis phases of this study took nearly two years, which was longer than originally projected during the proposal phase of the study. This length of time makes it difficult to know if the responses participants provided on their CUWiP survey remained an accurate depiction of their views as physics majors and their views of gender roles and goal affordance throughout the study.

The final limitation to this study was the use of the normative binary male and female gender categories. It is important to recognize that individuals with gender identities other than male and female participate in, and contribute to, male-dominated STEM fields and may experience their own set of relationships with the phenomena explored in this study. For this and other reasons explored during Chapter 5, the categories of experience that makeup the outcome space of the qualitative phase of this study should not be considered an exhaustive representation of the qualitatively different 
ways women and people of other genders experience the conceptual framework used to examine the phenomenon under investigation here.

\section{Implications}

As revealed throughout this study, the root and contributing factors to gender disparity in male-dominated STEM fields is complex, as is the development, implementation, and propagation of practices that affect change in this arena. The results of this study generate a number of implications for higher education faculty and administrators to consider when the recruitment and retention of women into physical science fields is the topic in focus. The three factors most salient for consideration here focus on the theoretical implications, the implications for faculty and academic programs, and the implications for practice by student affairs and other university administrators as found to be consistent with the results of this study and the literature.

\section{Theoretical Implications}

The findings of this study were able to address some of the new directions for research in the literature, such as Diekman et al.'s (2017) shifting focus on the qualitatively different communal roles and goals individuals play in their lives, often playing different roles in different goal pathways or spaces they occupy. They also call for additional research integrating the communal and agentic factors to better understand how those who, "are highly agentic and highly communal might be most likely to reach levels of excellence in STEM when communal opportunities are available" (p. 162). This study contributes to better understanding some of the qualitatively different ways undergraduate women navigate, manage, prioritize, integrate, and in some cases reject communality in their roles and goals. It is critical to examine the way integrating the 
agentic and communal dimensions of Diekman et al.'s (2010) gender congruity model influenced the findings of this study significantly, and the implications of these findings on utilizing this theory in future research.

The most salient finding of this study was the division of the agentic dimension of Diekman's theory into extrinsic agentic and intrinsic agentic goals early on in the statistical analysis. The finding aligns with previous findings indicating intrinsic motivation as predictive of STEM interest and success (Hazari et al., 2010; see also Diekman et al., 2017). Integrating intrinsic agency dimensions defined by individualism, independence, self-direction, and demonstrating skill into the analysis of women's identity development, success, and self-reported persistence plans in a male-dominated STEM field further contextualizes the multi-dimensional roles and goals women, and arguably anyone, negotiate and reconcile throughout their decisions to remain in or exit their chosen field. Researchers seeking to further contextualize the gender gap in maledominated fields should consider the lens of intrinsic agency for role it plays as motivation, identity development facilitator, and a bridge between the stereotypic perspectives of communality or extrinsic agency as opposing, mutually exclusive roles.

Further understanding the complexity of how these constructs interact in participants' experiences would also further inform related research. Qualitative data shed light on these theoretical complexities that helps us understand how to make change, such as reframing communality as an advantage in physics, rather than a disadvantage.

\section{Implications for Faculty and Academic Programs}

It was evident throughout the participant interviews that classroom and academic cultures vary widely across the U.S. This is not surprising. However a common thread 
through participants' examples - many of which included personal experience with, or knowledge of others' experiences only once or twice removed - indicates discriminatory and bias-motivated behaviors performed by other students, graduate students, postdoctoral researchers, and faculty in nearly every participant's experience, regardless of academic program culture type.

Faculty and academic affairs administrators are in positions of power with the responsibility to directly influence the culture and behaviors practiced within those cultures. This was further confirmed by the reflective category participants who time and again observed these behaviors with a heightened sense of awareness of the indiscretions at the time, but a consistent practice of ceding power to their professors, their graduate student mentors and supervisors, and their academic program chairs and deans. Some women in physics programs are asking for help and expecting results. These results can come in the form of faculty and administration cross-cultural training, curriculum reform according to gender-inclusive best-practices, and supporting student and faculty participation in programs such as CUWiP.

Perhaps a more granular approach is also available for consideration here. A few participants shared experiences in which they overheard other students discussing the way women and other minorities are taking opportunities from qualified students (e.g., acceptance to academic programs, scholarship dollars, etc.). Faculty and academic administrators should consider receiving the proper training, conducting their own research, empowering their colleagues and themselves to confront this behavior in direct, productive ways. Furthermore, faculty can and should be equipped with the skills to teach and empower other students to confront these behaviors when they observe them. 
Higher education classrooms, labs, and study spaces integrated into faculty spaces are an excellent opportunity to prepare students for professional, respectful behavior they will bring with them to their careers beyond their higher education experiences. Students are paying attention to the level of accountability those who perpetuate behaviors that directly contribute to a "chilly climate" are held to.

Finally, department chairs and deans with the "bird's eye" view of their department's culture, strengths, and challenges have a responsibility to see all of their students as possessing the potential to excel in their major and beyond. Program leaders need to be cognizant of how students with communal and extrinsic agentic goals can be supported and validated in their physics identities beyond encouraging participation in CUWiP. Inherent in this student-driven approach is department and program leadership's ability to create and amend policies and practices within their purview that encourages equitable recruitment and retention of faculty, researchers, and students. Just as students are watching how these practices are implemented by the faculty, students and faculty are also watching how department leadership navigates these issues, influences department culture, and reconciles inequities whenever possible.

\section{Implications for University Administrators}

Finally, the implications for administrators outside of the STEM departments directly influenced by the findings of this study are equally as critical. Scrutiny of the positioning of higher education institutions as inefficient, overly-bureaucratic, ultraliberal indoctrination hubs influences public perception of the value of a college degree while simultaneously serving as justification to redirect funds elsewhere in society. Therefore, with increased focus on retention efforts and dwindling public dollars in 
support of higher education, the pressure to provide the most successful, efficient programs that retain the most students and help them achieve viable careers is as formidable as ever. Student affairs professionals, academic and career advisors, and the highest levels of administrative leadership must consider these pressures while continuing their dedication (or rededicating themselves) to diversity and equality values into the vision, mission, and practices of the institution; as such, they must be cognizant of the issues in fields such as physics so that they can make targeted and effective recommendations to programs. Research-based interventions and support programs for STEM students produced outside of the classroom and academic department can provide immense support to the academic programs so they do not experience the responsibility of closing the gender gap in isolation.

Ensuring that fields dominated by any population have access to all students capable of contributing to the fields significantly in the future is ultimately the responsibility of every administrative level at the university. Physics is not the only male-dominated programs on campuses. Philosophy, economics, computer science, engineering, and mathematics are among some of the other academic fields consistently dominated by White males (Leslie at al., 2015), partially due to the assumptions about brilliance required for these fields, and partially because of the foundations and culture of these fields. Higher education portfolios inclusive of multiple fields of study dominated by any one type of person deserve attention and ongoing discussion with administrators and faculty in those areas to determine whether or not change should be incorporated into future goal setting and reporting cycles. Where change is not immediately possible due to limitations in student populations, understanding the experiences of underrepresented 
populations, such as women in physics, can prompt creative solutions such as increased diversity in guest speakers at campus events, and examining faculty and administrative hiring and promotion practices. Additionally, great opportunities exists for related training for academic and career advisors, who can have individually tailored conversations with women considering entering male-dominated fields, as well as those already in those majors. If academic and career advisors are well-prepared to determine if a student is considering changing her major because of performance challenges versus some of the barriers explored in this study, advisors have the potential to positively impact a student's willingness to develop knowledge, awareness, and skills that may instead give them what they need to persist.

\section{Recommendations for Future Research}

Although research on women in physics exists as a line of inquiry derivative of feminist science studies, the breadth is not exhaustive by any means. This section provides additional recommendations for research to further expand knowledge and understanding of the experience of women who persist in physics and the existing efforts to close the leadership gap in this field.

\section{On Grit}

One of the relatively surprising outcomes of this study was the prominence of grit as integral to participants' willingness and ability to ignore, reconcile, and otherwise navigate internal and external persistence barriers. Participants in the qualitative portion

of the study often cited grit, whether directly or indirectly, as a practice that allowed them to do things like talk themselves out of negative self-efficacy thought cycles, push past visible and invisible sexism, as a form of intrinsic motivation when studying and 
conducting research, and in seeking ways to connect with others despite the acute knowledge that their major challenged that at times; a study examining this nuance could unlock barriers for women and other underrepresented groups interested in STEM fields. It was determined that in order to incorporate as much of the conceptual framework guiding this study into the outcome space of Chapter 5, the topic of grit should be discussed as a sub-finding rather than as a theme or category of experience. However, grit yields many opportunities to consider how students develop awareness and skills related to grit; participants in this study shared many examples of how they make decisions to utilize grit as a useful tool, and the students who were aware that they possessed grit as a tool available to them seemed more likely to apply this tool in academic, research, professional, and social settings than those who could not articulate this.

\section{Incorporating Male and Non-Binary Gender Perspectives}

A study similar to this employing mixed methodology including participants identifying as male as well as participants identifying their gender as non-binary would contextualize these issues further, and provide a more robust perspective of the culture of physics in higher education today. Additionally, expanding the study beyond the U.S. would also offer additional opportunities for comparative understanding of equality and diversity issues in the field, while also increasing opportunities to understand bestpractices and cultural influences that have yielded gender equality. With greater understanding of the way male students understand and articulate the gender gap in their major, more productive training and interventions can be developed to influence the way men can contribute to closing the gap. Incorporating the way gender non-binary students navigate the complex components of their gender and physics identity could refine 
training and development approaches incorporated into efforts to close the gender gap in physics further.

\section{Highly Communal/Highly Agentic Success in STEM}

Related to the findings of this study, and echoing the call for further research on this topic in Diekman et al. (2017), women of color responding to the CUWiP survey questions about communal and agentic goals scored high across these dimensions. This may be because their underrepresented position has required them to have already developed their physics identity and skill sets that facilitate their own success by the time they get to college and choose physics as their major. This position may also require knowledge and skills across the various gender role dimensions in order for them to adapt and be successful across various contexts.

In this same vein, some of the students who were highly communal and highly agentic articulated clearly communicated the way they have incorporated communal goals and roles into their experiences, or found success in academic program cultures that valued collaborative, communal roles visibly. Further research to better understand the way STEM majors and professionals reconcile their highly agentic and highly communal roles and goals could offer additional strategies for educating children and young adults about the ways physics, engineering, and other STEM fields afford time and space for communality, which could positively influence recruitment efforts aimed to increase female participation in STEM overall, and male-dominated STEM fields specifically. Also, further qualitative studies of how non-persisters at the bachelor's degree level navigate their communal and agentic goals may help us to better understand how failures 
to resolve incongruences happen, and how these students understand their departure from the field.

\section{Summary}

This chapter discussed the findings for each of the research questions under examination for this study, as well as the interpretation and discussion of the inferences drawn from the mixed methods approach taken. The limitations, implications for theory, research, and practice, and future research recommendations were presented.

This dissertation examined the physics identity, gender role congruity, perceptions of the "ideal" scientist, and self-reported persistence plans for undergraduate women majoring in the male-dominated academic fields of physics through a sequential explanatory mixed methods design. The quantitative findings presented in Chapter 4 began by splitting the agentic goal dimension of Diekman et al.'s (2010) gender role congruity into extrinsic and intrinsic agentic goals. Then, answers to the quantitative research questions included significant correlations such as physics identity as a significant indicator of persistence plans across a student's career, communal goals as negatively correlated with bachelor's degree completion, and intrinsic agentic goals correlated with graduate school and career plans. It was determined that participants in the qualitative phase experience the phenomenon of being a female in a male-dominated physics major in qualitatively different ways, resulting in five distinct categories of experience, namely: (a) the assured; (b) the solitary; (c) the communal; (d) the reflective; (e) and, the ambassadors. These categories of experience further explain the quantitative findings by delineating the depths of the differences and similarities in participants' experiences with the conceptual framework. 
Among the best examples of the explanatory nature of the qualitative findings is the way the communal category participants framed their future plans in expressly communal terms such as connecting with others, feeling supported by others, and seeking graduate school and career plans that allow them to help others tangibly. Examining the nuanced ways in which their communal roles and goals are shaped by the way they anticipate and experience communal social roles illuminates the negative correlation between communal goals and bachelor's degree completion plans in complex ways. Higher education practitioners and faculty must take stock of the practices and campus cultures that influence or perpetuate gender disparity in physics departments and other departments experiencing population imbalances. The findings of this study serve as additional information aimed at informing faculty and administration policies and practices that encourage positive change resulting in gender equality in male-dominated academic programs such as physics. If additional attention and effort is paid to how physics departments recruit and retain student populations that include more women, the ways women can contribute to this field in the future will likely be significant. 


\section{REFERENCES}

American Association for the Advancement of Science (AAAS). (2005). AAAS board statement on women in science and engineering. Retrieved June 2, 2015 from http://archives.aaas.org/docs/resolutions.php?doc_id=439

American Physical Society. CUWiP 2016 Advertisement. Retrieved May 27, 2015 from http://www.aps.org/programs/women/workshops/upload/2016-CUWIP-Flyer.pdf

Allan, E. J., \& Madden, M. (2003, April). Chilly classrooms for female undergraduate students at a research university: A question of method? Paper presented at the Annual Meeting of the American Educational Research Association, Chicago, Illinois.

Allport, G. W. (1979). The nature of prejudice. New York, NY: Basic Books.

Anfara, V. A., \& Mertz, N. T. (Eds.). (2006). Theoretical frameworks in qualitative research. Thousand Oaks, CA: Sage.

Archer, L., DeWitt, J., Osborne, J., Dillon, J., Willis, B., \& Wong, B. (2012). "Balancing acts": Elementary school girls' negotiations of femininity, achievement, and science. Science Education, 96, 967-989.

Archer, L., DeWitt, J., Osborne, J., Dillon, J., Willis, B., \& Wong, B. (2013). 'Not girly, not sexy, not glamorous': primary school girls' and parents' constructions of science aspirations 1. Pedagogy, Culture \& Society, 21, 171-194.

Akerlind, G. (2012). Variation and commonality in phenomenographic research methods. Higher Education Research \& Development, 13, 115-127.

Auerback, C.F., \& Silverstein, L.B. (2003). Qualitative data: An introduction to coding and analysis. New York: New York University Press.

Baker, D. (2013). What works: Using curriculum and pedagogy to increase girls' interest and participation in science. Theory Into Practice, 52, 14-20.

Bandura, A. (1997). Self-efficacy: The exercise of control. New York, NY: Freeman.

Barman, J. (2017, August 6). Google employee writes internal memo railing against women, diversity; rage ensues. SFist: News, Food, Arts \& Events. Retrieved from http://sfist.com

Barton, A. C., \& Tan, E. (2010). We be burnin'! Agency, identity, and science learning. The Journal of the Learning Sciences, 19, 187-229. 
BBC News (June 11, 2015). Sir Tim Hunt resigns from university role over girls comments. BBC News.com. Retrieved from http://www.bbc.com/news/uk33090022

Beddoes, K., \& Borrego, M. (2011). Feminist theory in three engineering education journals, 1995-2008. Journal of Engineering Education, 100, 281-303.

Belenky, M. F., Clinchy, B. M., Goldberger, N. R., \& Tarule, J. M. (1986). Women's ways of knowing: The development of self, voice, and mind. New York, NY: Basic Books.

Bem, S. L. (1981). Gender schema theory: A cognitive account of sex typing. Psychological Review, 88, 354-364.

Bian, L., Leslie, S. J., \& Cimpian, A. (2017). Gender stereotypes about intellectual ability emerge early and influence children's interests. Science, 355, 389-391.

Brickhouse, N. W. (2001). Embodying science: A feminist perspective on learning. Journal of Research in Science Teaching, 38, 282-295.

Brickhouse, N. W., Lowery, P., \& Schultz, K. (2000). What kind of girl does science? The construction of school science identities. Journal of Research in Science Teaching, 37, 441-458.

Bogdan, R.C., \& Biklen, S.K. (2007). Qualitative research for education: An introduction to theories and methods $\left(5^{\text {th }}\right.$ ed.). Boston, MA: Pearson Education.

Brotman, J. S., \& Moore, F. M. (2008). Girls and science: A review of four themes in the science education literature. Journal of Research in Science Teaching, 45, 9711002 .

Brower, A., \& Inkelas, K.K. (2007). National study of living-learning programs: National report. Sponsored by: NSA, ACUHO-I, ACPA, NASPA. Retrieved from http://undergraduate.uncg.edu/about/forms/2007_NSLLP_Final_Report[1].pdf

Bystydzienski, J. M., \& Brown, A. (2012). "I just want to help people”: Young women's gendered engagement with engineering. Feminist Formations, 24(3), 1-21.

Callahan, J., Pyke, P., Shadle, S., \& Landrum, R. E. (2014, June). Creating a STEM identity: Investment with return. Paper session presented at the $121^{\text {st }}$ ASEE Annual Conference \& Exposition, Indianapolis, IN.

Carlone, H. B. (2004). The cultural production of science in reform-based physics: Girls' access, participation, and resistance. Journal of Research in Science Teaching, $41,392-414$. 
Carlone, H. B., \& Johnson, A. (2007). Understanding the science experiences of successful women of color: Science identity as an analytic lens. Journal of Research in Science Teaching, 44, 1187-1218.

Ceci, S. J., \& Williams, W. M. (2010). The mathematics of sex: How biology and society conspire to limit talented women and girls. New York: Oxford University Press.

Ceci, S. J., \& Williams, W. M. (2011). Understanding current causes of women's underrepresentation in science. Proceedings of the National Academy of Sciences, 108, 3157-3162.

Ceci, S. J., Williams, W. M., \& Barnett, S. M. (2009). Women's underrepresentation in science: Sociocultural and biological considerations. Psychological Bulletin, 135, $218-261$.

Chang, M. J., Eagan, M. K., Lin, M. H., \& Hurtado, S. (2011). Considering the impact of racial stigmas and science identity: Persistence among biomedical and behavioral science aspirants. The Journal of Higher Education, 82, 564-596.

Charmaz, K. (2006). Constructing grounded theory: A practical guide through qualitative analysis. Thousand Oaks, CA: Sage.

Chase, S.E. Narrative inquiry: Multiple lenses, approaches, voices. (2005). In N. Denzin \& Y. Lincoln (Eds.), The Sage Handbook of Qualitative Research ( $3^{\text {rd }}$ ed, pp. 651-679.). Thousand Oaks, CA: Sage.

Chen, X. (2009). Students who study science, technology, engineering, and mathematics (STEM) in postsecondary education. NCES 2009-161. National Center for Education Statistics. Washington, D.C.

Cheryan, S., Master, A., \& Meltzoff, A. N. (2015). Cultural stereotypes as gatekeepers: increasing girls' interest in computer science and engineering by diversifying stereotypes. Frontiers in Psychology, 6(49), 1-8.

Cho, S., Crenshaw, K. W., \& McCall, L. (2013). Toward a field of intersectionality studies: Theory, applications, and praxis. Signs. 38, 785-810.

Clance, R. R., \& Imes, S. (1978). The imposter phenomenon in high achieving women: dynamics and therapeutic intervention. Psychotherapy Theory, Research and Practice, 15(3), 1-9.

Clancy, E. P. (1962). Women and physics. American Journal of Physics, 30, 626-629.

Clandinin, D. J., \& Connelly, F. M. (2000). Narrative inquiry: Experience and story in qualitative research. San Fancisco: Jossey-Bass. 
Clark Blickenstaff, J. (2005). Women and science careers: Leaky pipeline or gender filter?. Gender and Education, 17, 369-386.

Cleaves, A. (2005). The formation of science choices in secondary school. International Journal of Science Education, 27, 471-486.

Charmaz, K. (2006). Constructing grounded theory: A practical guide through qualitative analysis. Thousand Oaks, CA: Sage.

Cobb, P. (2004). Mathematics, literacies, and identity. Reading Research Quarterly, 39, 333-337.

Cole, D. \& Espinoza, A. (2008). Examining the academic success of Latino students in science technology engineering and mathematics (STEM) majors. Journal of College Student Development, 49, 285-300.

Collins, P.H. (2009). "Foreword: Emerging intersections - Building knowledge and transforming institutions." In B. T. Dill and R. E. Zambrana (Eds.), Emerging intersections: Race, class, and gender in theory, policy, and practice. New Brunswick, NJ: Rutgers University Press.

Conger, K. (2017, August 5) Exclusive: Here's the full 10-page anti-diversity screed circulating internally at Google [updated]. Gizmodo. Retrieved from http://gizmodo.com/

Connelly, F. M., \& Clandinin, D. J. (1990). Stories of experience and narrative inquiry. Educational Researcher, 19(5), 2-14.

Crenshaw, K. (1991). Mapping the margins: Intersectionality, identity politics, and violence against women of color. Stanford Law Review, 1241-1299.

Creswell, J. W. (2007). Qualitative inquiry \& research design: Choosing among five approaches (2nd ed.). Thousand Oaks, CA: Sage.

Creswell, J.W. (2014). Research design: Qualitative, quantitative, and mixed methods approaches $\left(4^{\text {th }}\right.$ ed.). Los Angeles, CA: Sage.

Creswell, J.W. (2015). A concise introduction to mixed methods research. Los Angeles, CA: Sage.

Danielsson, A. T. (2009). Doing physics--doing gender: An exploration of physics students' identity constitution in the context of laboratory work (Doctoral thesis, Uppsala University, Uppsala Sweden). Retrieved from https://books.google.com/ books?hl=en\&lr=\&id=E6DLc3pjuYC\&oi=fnd\&pg=PP4\&ots=04o32 V24en\&sig= 2XveygbqUwL9q8_xTuY1dpoj7s\#v=onepage\&q\&f=false 
De Beauvoir, S. (2012). The second sex. New York, NY: Vintage Books.

Denzin, N. K. (1997). Interpretive ethnography: Ethnographic practices for the $21^{\text {st }}$ century. Thousand Oaks, CA: Sage.

Denzin, N.K., \& Lincoln, Y.S. (2003). The landscape of qualitative research ( $\left.2^{\text {nd }} \mathrm{ed}.\right)$. Thousand Oaks, CA: Sage.

Diekman, A. B., Brown, E. R., Johnston, A. M., \& Clark, E. K. (2010). Seeking congruity between goals and roles: A new look at why women opt out of science, technology, engineering, and mathematics careers. Psychological Science, 21, 1051-1057.

Diekman, A. B., Clark, E. K., Johnston, A. M., Brown, E. R., \& Steinberg, M. (2011). Malleability in communal goals and beliefs influences attraction to stem careers: Evidence for a goal congruity perspective. Journal of personality and social psychology, 101, 902-918.

Diekman, A. B., \& Eagly, A. H. (2000). Stereotypes as dynamic constructs: Women and men of the past, present, and future. Personality and Social Psychology, 26, 11711188.

Diekman, A. B., \& Goodfriend, W. (2006). Rolling with the changes: A role congruity perspective on gender norms. Psychology of Women Quarterly, 30, 369-383.

Diekman, A. B., \& Steinberg, M. (2013). Navigating social roles in pursuit of important goals: A communal goal congruity account of STEM pursuits. Social and Personality Psychology Compass, 7, 487-501.

Diekman, A. B., Steinberg, M., Brown, E. R., Belanger, A. L., \& Clark, E. K. (2017). A goal congruity model of role entry, engagement, and exit: Understanding communal goal processes in STEM gender gaps. Personality and Social Psychology Review, 21, 142-175.

Dunn, C. M., \& Chadwick, G. L. (2002). Protecting study volunteers in research $\left(2^{\text {nd }}\right.$ ed.). Boston, MA: Centerwatch.

Dyer, S. K. (2004). Under the microscope: A decade of gender equity projects in the sciences. American Association of University Women Educational Foundation, 1111 Sixteenth St. NW, Washington, DC 20036.

Eagly, A. H., \& Diekman, A. B. (2005). What is the problem? Prejudice as an attitude-incontext. In J. F. Dovidio, P. Glick, \& L. A. Rudman (Eds.), On the nature of prejudice: Fifty years after Allport (pp. 19-35). Malden, MA: Blackwell. 
Eagly, A. H., \& Karau, S. J. (2002). Role congruity theory of prejudice toward female leaders. Psychology Review, 109, 573-598.

Eagly, A. H., Wood, W., \& Diekman, A. B. (2000). Social role theory of sex differences and similarities: A current appraisal. In T. Eckes \& H. M. Trautner (Eds.), The developmental social psychology of gender (pp. 123-174). Mahwah, NJ: Lawrence Erlbaum Associates.

Etzkowitz, H., Kemelgor, C., \& Uzzi, B. (2000). Athena unbound: The advancement of women in science and technology. Cambridge University Press.

Epstein, D. (2006). So that's why they're leaving. Inside Higher Education. Retrieved from https://www.insidehighered.com/news/2006/07/26/scipipeline

Espinosa, L. L. (2011). Pipelines and pathways: Women of color in undergraduate STEM majors and the college experiences that contribute to persistence. Harvard Educational Review, 81, 209-240.

Farenga, S. J., \& Joyce, B. A. (1999). Intentions of young students to enroll in science courses in the future: An examination of gender differences. Science Education, 83, 55-75.

Fassinger, R. E., \& Asay, P. A. (2006). Career counseling for women in science, technology, engineering, and mathematics (STEM) fields. In W. B. Walsh \& M. J. Heppener (Eds.), Handbook of career counseling for women $\left(2^{\text {nd }}\right.$ ed., pp. 427452). Mahwah, NJ: Lawrence Erlbaum.

Faulkner, W. (2001). The technology question in feminism: A view from feminist technology studies. Women's Studies International Forum, 24, 79-95.

Faulkner, W. (2007). 'Nuts and bolts and people': Gender-troubled engineering identities. Social Studies of Science, 37, 331-356.

Feltman, R. (2016, January 13). Astronomy's snowballing sexual harassment scandal picks up even more cases. The Washington Post. Retrieved from https://www.washingtonpost.com/news/speaking-ofscience/wp/2016/01/13/astronomys-snowballing-sexual-harassment-scandalpicks-up-even-more-cases/

Gardiner, I. (2013, August 5). NexGeneGirls: Inspiring a new generation of women scientists. Boys \& Girls Clubs of San Francisco. Retrieved from http://www.kidsclub.org/nexgenegirls/

Gee, J.P. (2000). Identity as an analytic lens for research in education. Review of Research in Education, 25, 99-125. 
Ghorayshi, A. (2015, October 9). Famous Berkeley astronomer violated sexual harassment policies over many years. BuzzFeed News. Retrieved from http://www.buzzfeed.com/azeenghorayshi/famous-astronomer-allegedly-sexuallyharassed-students

Glaser, B. G. (1965). The constant comparative method of qualitative analysis. Social Problems, 12, 436-445.

Gilligan, C. (1982/1993). In a different voice: Psychological theory and women's development. Cambridge, MA: Harvard University Press. (Original work published in 1982).

Goldenberg, S. (2005). Why women are poor at science, by Harvard president. The Guardian. Retrieved from http://www.theguardian.com/science/2005/jan/18/educationsgendergap.genderiss ues

Gonsalves, A. J. (2014). "Physics and the girly girl-there is a contradiction somewhere": Doctoral students' positioning around discourses of gender and competence in physics. Cultural Studies of Science Education, 9, 503-521.

Götschel, H. (2014). No space for girliness in physics: understanding and overcoming the masculinity of physics. Cultural Studies of Science Education,9, 531-537.

Griffith, A. L. (2010). Persistence of women and minorities in STEM field majors: Is it the school that matters? Economics of Education Review, 29, 911-922.

Hall, J. A., \& Carter, J. D. (1999). Gender-stereotype accuracy as an individual difference. Journal of Personality and Social Psychology, 77, 350-359.

Hall, R. M., \& Sandler, B. R. (1982). The classroom climate: A chilly climate for women? Washington, D. C.: Association of American Colleges.

Hazari, Z., Cass, C. \& Beattie, C. (2015). Obscuring power structures in the physics classroom: Positioning, student engagement, and physics identity development. Journal of Research in Science Teaching, 52, 735-762.

Hazari, Z., Sadler, P. M., \& Sonnert, G. (2013). The science identity of college students: exploring the intersection of gender, race, and ethnicity. Journal of College Science Teaching, 42, 82-91.

Hazari, Z., Sonnert, G., Sadler, P.M., \& Shanahan, M-C. (2010). Connecting high school physics experiences, outcome expectations, physics identity, and physics career choice: a gender study. Journal of Research in Science Teaching, 47, 978-1003. 
Hazari, Z., Tai, R.H., \& Sadler, P.M., (2007). Gender differences in introductory university physics performance: The influence of high school physics preparation and affective factors. Science Education, 91, 847- 876.

Hendley, A., \& Charles, M. (2015). Gender segregation in higher education. Emerging trends in the social and behavioral sciences: An interdisciplinary, searchable, and linkable resource.

Howell, D. C. (2007). Statistical methods for psychology. (6 ${ }^{\text {th }}$ ed.). Belmont, CA: Thomson.

Hughes, R. M. (2010). Keeping university women in STEM fields. International Journal of Gender, Science and Technology 2, 417-436.

Jones, J. (2010). Closing the gender gap. Civil Engineering Magazine Archive, 80(07), 60-63.

Jones, M. G., Howe, A., Rua, M. J. (2000). Gender differences in students' experiences, interests, and attitudes towards science and students. Science Education, 84, 180-192.

Jones, S. R., \& Wijeyesinghe, C. L. (2011). The promises and challenges of teaching from an intersectional perspective: Core components and applied strategies. New Directions for Teaching and Learning, 2011(125), 11-20.

Johnson, A. C. (2007). Unintended consequences: How science professors discourage women of color. Science Education, 91, 805-821.

Johnson, D. R. (2012). Campus racial climate perceptions and overall sense of belonging among racially diverse women in STEM majors. Journal of College Student Development, 53, 336-346.

Johnston, A. M., \& Diekman, A. B. (2015). Pursuing desires rather than duties? The motivational content of gender stereotypes. Sex Roles, 73(1-2), 16-28.

Jorgenson, J. (2002). Engineering selves: Negotiating gender and identity in technical work. Management Communication Quarterly 15, 350-380.

Joyce, B. A., \& Farenga, S. J. (1999). Informal science experience, attitudes, future interest in science, and gender of high-ability students: An exploratory study. School Science and Mathematics, 99, 431-437.

Kane, J. M. (2012). Young African American children constructing academic and disciplinary identities in an urban science classroom. Science Education, 96, 457487. 
Kenway, J., \& Gough, A. (1998). Gender and science education in schools: A review "with attitude." Studies in Science Education, 31, 1-30.

Knorr-Cetina, K. (1999). Epistemic cultures: How the sciences make knowledge. Cambridge, MA: Harvard University Press.

King, N., \& Horrocks, C. (2010). Interviews in qualitative research. Thousand Oaks, CA: Sage.

Kilson, M. (1976). The status of women in higher education. Signs, 1(4), 935-942.

Leslie, S.-J., Cimpian, A., Meyer, M., \& Freeland, E. (2015). Expectations of brilliance underlie gender distributions across academic disciplines. Science, 347(6219), 262-265.

MacQueen, K. M., McLellan-Lemal, E., Bartholow, K., \& Milstein, B. (2008). Teambased codebook development: Structure, process, and agreement. In G. Guest \& K. M. MacQueen (Eds.), Handbook for team-based qualitative research (pp. 119$135)$.

Martin, B. R., \& Irvine, J. (1982). Women in science - The astronomical brain drain. Women's Studies International Forum, 5, 41-68.

Marton, F. (1981). Phenomenography - describing conceptions of the world around us. Instructional Science, 10, 177-200.

Marton, F. (1986). Phenomenography - a research approach to investigating different understandings of reality. Journal of Thought, 21(3), 28-49.

Marton, F., \& Booth, S. (1997). Learning and awareness. New York, NY: Routledge.

Mcall, L. (2005). The complexity of intersectionality. Signs. 30, 1771-1800.

McCarthy, J. L. \& Wolfe, D. (1975). Doctorates granted to women and minority group members. Science, 189(4026), 856-859.

McPherson, W., \& Fuselier-Thompson, D. R. (2013). Minority women in STEM: A valuable resource in the global economy. Journal of the International Association for the Study of the Global Achievement Gap, 1(Book 5), 45-58.

Merriam, S. B. (2014). Qualitative research: A guide to design and implementation. San Francisco, CA: Jossey-Bass.

Miles, M. B., \& Huberman, A. M. (1994). Qualitative data analysis $\left(2^{\text {nd }}\right.$ ed.). Thousand Oaks, CA: Sage. 
Miller, D. I., Eagly, A. H., \& Linn, M. C. (2015). Women's representation in science predicts national gender-science stereotypes: Evidence from 66 nations. Journal of Educational Psychology, 107, 631-644.

Morse, J. M., \& Niehaus, L. (2009). Mixed methods design: Principles and procedures. Walnut Creek, CA: Sage.

Moulton Belec, H. (2015). Princess or geek: What attracts women to science? Outlook. $109,18-21$.

Nasir, N. (2002). Identity, goals, and learning: Mathematics in cultural practice. Mathematical Thinking and Learning, 4, 211-245.

National Science Board. (2016). Science and Engineering Indicators 2016. Arlington, VA: National Science Foundation (NSB-2016-1).

National Science Foundation. (2007). Women, minorities, and persons with disabilities in science and engineering. National Science Foundation, Division of Science Resources Statistics, Arlington, VA: NSF07-315.

National Science Foundation. (2014). Chapter 2: Higher education in science and engineering. Science and Education Indicators 2014. NSF. Retrieved May 23, 2015 from http://www.nsf.gov/statistics/seind14/index.cfm/chapter-2/c2h.htm

National Science Foundation. (2015). Women, minorities, and persons with disabilities in science and engineering: 2015. National Center for Science and Engineering Statistics \& Directorate for Social, Behavioral and Economic Sciences. Retrieved May 23, 2015 from http://www.nsf.gov/statistics/2015/nsf15311/digest/nsf15311digest.pdf

National Science Foundation. (2015). American Physical Society (APS) IPEDS completion survey data. Retrieved July 29, 2017 from https://www.aps.org/programs/education/statistics/

Nicholson, S. E., \& Pasque, P. A. (2011). An introduction to feminism and feminist perspectives in higher education and student affairs. In P. A. Pasque, \& S. E. Nicholson (Eds.), Empowering Women in Higher Education and Student Affairs: Theory, Research, Narratives, and Practice from Feminist Perspectives (pp. 314). Sterling, Virginia: Stylus Publishing.

Niskode-Dossett, Pasque, P. A., \& Nicholson, S. E. (2011). Envisioning a new future with feminist voices: Research and practice from feminist perspectives. In P. A. Pasque, \& S. E. Nicholson (Eds.), Empowering Women in Higher Education and Student Affairs: Theory, Research, Narratives, and Practice from Feminist Perspectives (pp. 3-14). Sterling, Virginia: Stylus Publishing. 
Obama, B. (2009). President Barak Obama addresses the $146^{\text {th }}$ Annual Meeting of the National Academy of Sciences. Proceedings of the National Academy of Sciences, 106(24), 9439-9543.

Obama, B. (2013, February). Women in STEM [White House Office of Science and Technology Policy]. Retrieved from https://www.whitehouse.gov/administration/eop/ostp/women

Oh, S. S. \& Lewis, G. B. (2011). Stemming inequality? Employment and pay of female and minority scientists and engineers. Social Science Journal, 48, 397-403.

Osborne, J. W., Costello, A. B., \& Kellow, J. T. (2008). Best practices in exploratory factor analysis. In J. W. Osborne (Ed.), Best practices in quantitative methods (pp. 86-99). Los Angeles, CA: Sage.

Parsons, E. C. (1997). Black high school females' images of the scientist: Expression of culture. Journal of Research in Science Teaching, 34, 745-768.

Peng, S. S., \& Jaffe, J. (1979). Women who enter male-dominated fields of study in higher education. American Educational Research Journal, 16, 285-293.

Perna, L., Lundy-Wagner, V., Drezner, N. D., Gasman, M., Yoon, S., Bose, E., \& Gary, S. (2009). The contribution of HBCUs to the preparation of African American women for STEM careers: A case study. Research in Higher Education, 50, 1-23.

Potvin, G., Hazari, Z., \& Lock, R. M. (2014). Exposure to underrepresentation discussion: The impacts on women's attitudes and identities. Retrieved from The comPADRE Pathway on June 2, 2015 http://www.compadre.org/per/perc/2014/files/PERC2014UnderrepresentationExp erimentResubmission2.pdf

Rahm, J. (2007). Urban youths' identity projects and figured worlds: Case studies of youths' hybridization in science practices at the margin. Chicago, IL: The Chicago Springer Forum, Science Education in an Age of Globalization.

Rosenbaum, E. (2013, November 20). GoldieBlox ad perpetuates the fallacy of the pink. Ms. Magazine. Retrieved from: http://msmagazine.com/blog/2013/11/20/goldieblox-ad-perpetuates-the-fallacyof-the-pink/

Rosenthal, L., London, B., Levy, S. R., \& Lobel, M. (2011). The roles of perceived identity compatibility and social support for women in a single-sex STEM program at a co-educational university. Sex Roles, 65, 725-736. 
Rubin, H.J., \& Rubin, I.S. (2012). Qualitative interviewing: The art of hearing data ( $3^{\text {rd }}$ ed.). Los Angeles, CA: Sage.

Sader, J. (2011). The influence of gender: A conceptual model from women doctoral students in computer science. In P. A. Pasque, \& S. E. Nicholson (Eds.), Empowering women in higher education and student affairs: Theory, research, narratives, and practice from feminist perspectives (pp. 3-14). Sterling, Virginia: Stylus Publishing.

Sadler, P. M., Sonnert, G., Hazari, Z. \& Tai, R. (2012). Stability and volatility of STEM career interest in high school: A gender study. Science Education, 96, 411-427.

Salndaña, J. (2013). The coding manual for qualitative researchers $\left(2^{\text {nd }}\right.$ ed.). Los Angeles, CA: Sage.

Salter, D. W. (2003). Exploring the "chilly classroom" phenomenon as interactions between psychological and environmental types. Journal of College Student Development, 44, 110-121.

Salto, L. M., Riggs, M. L., Delgado De Leon, D., Casiano, C. A., \& De Leon, M. (2014). Underrepresented Minority High School and College Students Report STEMPipeline Sustaining Gains After Participating in the Loma Linda University Summer Health Disparities Research Program. PLoS ONE, 9(9), e108497. http://doi.org/10.1371/journal.pone.0108497

Sandler, B. R., Silverberg, L. A. \& Hall, R. M. (1996). The chilly classroom climate: A guide to improve the education of women. Washington, DC: National Association for Women in Education.

Sansone, C., \& Harackiewicz, J. M. (2000). Intrinsic and extrinsic motivation: The search for optimal motivation and performance. Academic Press: San Diego.

Sax, L. (1994). Retaining tomorrow's scientists: Exploring the factors that keep male and female college students interested in science careers. Journal of Women and Minorities in Science and Engineering, 1, 45-61.

Seidman, I. (2013). Interviewing as qualitative research: A guide for researchers in education and the social sciences. Teachers College Press: New York.

Shanahan, M.-C. (2007) Playing the role of science student: Exploring the meaning of the science student role and its relationship to students' identification in science. Doctoral dissertation, Ontario Institute for Studies in Education, University of Toronto. 
Shanahan, M.-C., \& Nieswandt, M. (2010). Science student role: Evidence of social structural norms specific to school science. Journal of Research in Science Teaching, 48, 367-395.

Shapiro, C. A., \& Sax, L. J. (2011). Major selection and persistence for women in STEM. New Directions for Institutional Research, 152, 5-18.

Slomka, J., McCurdy, S., Ratliff, E. A., Timpson, S., \& Williams, M. L. (2007). Perceptions of financial payment for research participation among AfricanAmerican drug users in HIV studies. Journal of General Internal Medicine, 22, 1403-1409.

Solomon, J. (1997). Girls' science education: Choice, solidarity and culture. International Journal of Science Education, 19, 407-417.

Soldner, M., Rowan-Kenyon, H., Inkelas, K. K., Garvey, J., \& Robbins, C. (2012). Supporting students' intentions to persist in STEM disciplines: The role of livinglearning programs among other social cognitive factors. The Journal of Higher Education, 83, p. 311-336.

Steele, J., James, J. B., \& Chait Barnett, R. (2002). Learning in a man's world: Examining the perceptions of undergraduate women in male-dominated academic areas. Psychology of Women Quarterly, 26, 46-50.

Svensson, L. (1997). Theoretical foundations of phenomenography. Higher Education \& Development 16, 159-171.

Szelenyi, K., Denson, N., \& Inkelas, K. K. (2013). Women in STEM majors and professional outcome expectations: The role of living-learning programs and other college environments. Research in Higher Education, 54, 851-873.

Tabachnick, B. G., \& Fidell, L. S. (2007). Using multivariate statistics (fith ed.). Boson, MA: Pearson.

Tashakkori, A., \& Teddlie, C. (1998). Mixed methodology: Combining qualitative and quantitative approaches (Vol. 46). Sage.

Tashakkori, A., \& Teddlie, C. (2003). Handbook of mixed methods in social \& behavioral research. Thousand Oaks, California: Sage.

Tong, R. (2009). Feminist thought: A more comprehensive introduction. Boulder, CO: West View Press.

Tonso, K. L. (2006). Student engineers and engineer identity: Campus engineer identities as figured world. Cultural Studies of Science Education, 1, 237-307. 
Toven-Lindsey, B., Levis-Fitzgerald, M., Barber, P. H., \& Hasson, T. (2015). Increasing persistence in undergraduate science majors: A model for institutional support of underrepresented students. CBE Life Sciences Education, 14(2), ar12. http://doi.org/10.1187/cbe.14-05-0082

Tracy, S. (2010). Qualitative quality: Eight "big-tent" criteria for excellent qualitative research. Qualitative Inquiry, 16(10), 837-851. Retrieved September 28, 2014 from: http://qix.sagepub.com/content/16/10/837.short

Trahar, S. (2009). Beyond the story itself: Narrative inquiry and autoethnography in intercultural research in higher education. Forum: Qualitative Social Research, 10(1), Art. 30-Art. 30.

University of Oklahoma. Center for Institutional Data Exchange and Analysis, \& Smith, T. Y. (2000). 1999-2000 SMET Retention Report: The Retention and Graduation Rates of 1992-98 Entering Science, Mathematics, Engineering and Technology Majors in 119 Colleges and Universities. University of Oklahoma.

Urry, M. (2003). Speeding up the long slow path to change. APS News, 12(2). Retrieved from https://cswa.aas.org/status/2003/JANUARY2003/SpeedingUpChange.html

Valian, V. (1999). Why so slow? The advancement of women. Cambridge, MA: Massachusetts Institute of Technology.

Winzenburg, S. (2011, March 2). How Skype is changing the interview process. The Chronicle of Higher Education. Retrieved from https://www.chronicle.com/article/How-Skype-Is-Changing-the/126529

Zeldin, A.L., \& Pajares, F. (2000). Against the odds: Self-efficacy beliefs of women in mathematical, scientific, and technological careers. American Educational Research Journal 37, 215-246. 


\section{APPENDICES}

\section{Appendix A: CUWiP IRB Approval Letter}

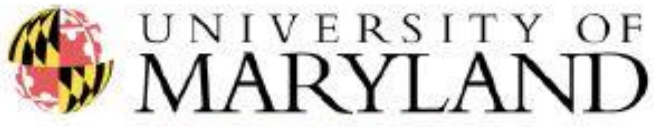

DNSTITUTIONAL RFVIEW BOARD
1204 Marie Mount Hall Colleze Part, MD 20742-5125 TEL 301.405 .4212 FAX 301.314 .1475 $\mathrm{irb} / \mathrm{a}=\mathrm{m}$ odu

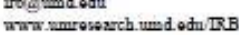

DATE:

TO:

FROM:

PROJECT TITLE:

REFERENCE \#:

SUBMISSION TYPE:

ACTION:

APPROVAL DATE:

EXPIRATION DATE: REVIEW TYPE:

REVIEW CATEGORY:
September 30,2015

Renee Michelle Goertzen, PhD

University of Maryland College Park (UMCP) IRB

[505475-10] Conferences for Undergraduate Women in Physics

Amendment/Modification

APPROVED

September 30,2015

September 11, 2016

Expedited Review

Expedited review category \# 7

Thank you for your submission of Amendment/Modification materials for this project. The University of Maryland College Park (UMCP) IRB has APPROVED your submission. This approval is based on an appropriate risk/benefit ratio and a project design wherein the risks have been minimized. All research must be conducted in accordance with this approved submission.

Prior to submission to the IRB Office, this project received scientific review from the departmental IRB Liaison.

This submission has received Expedited Review based on the applicable federal regulations.

This project has been determined to be a Minimal Risk project. Based on the risks, this project requires continuing review by this committee on an annual basis. Please use the appropriate forms for this procedure. Your documentation for continuing review must be received with sufficient time for review and continued approval before the expiration date of September 11, 2016.

Please remember that informed consent is a process beginning with a description of the project and insurance of participant understanding followed by a signed consent form. Informed consent must continue throughout the project via a dialogue between the researcher and research participant. Unless a consent waiver or alteration has been approved, Federal regulations require that each participant receives a copy of the consent document.

Please note that any revision to previously approved materials must be approved by this committee prior to initiation. Please use the appropriate revision forms for this procedure.

All UNANTICIPATED PROBLEMS involving risks to subjects or others (UPIRSOs) and SERIOUS and UNEXPECTED adverse events must be reported promptly to this office. Please use the appropriate reporting forms for this procedure. All FDA and sponsor reporting requirements should also be followed.

All NON-COMPLIANCE issues or COMPLAINTS regarding this project must be reported promptly to this office. 
Please note that all research records must be retained for a minimum of seven years after the completion of the project.

If you have any questions, please contact the IRB Office at 301-405-4212 or irb@umd.edu. Please include your project title and reference number in all correspondence with this committee.

This letter has been electronically signed in accordance with all applicable regulations, and a copy is retained within University of Maryland College Park (UMCP) IRB's records. 
Appendix B: CUWiP 2016 Event Advertisement

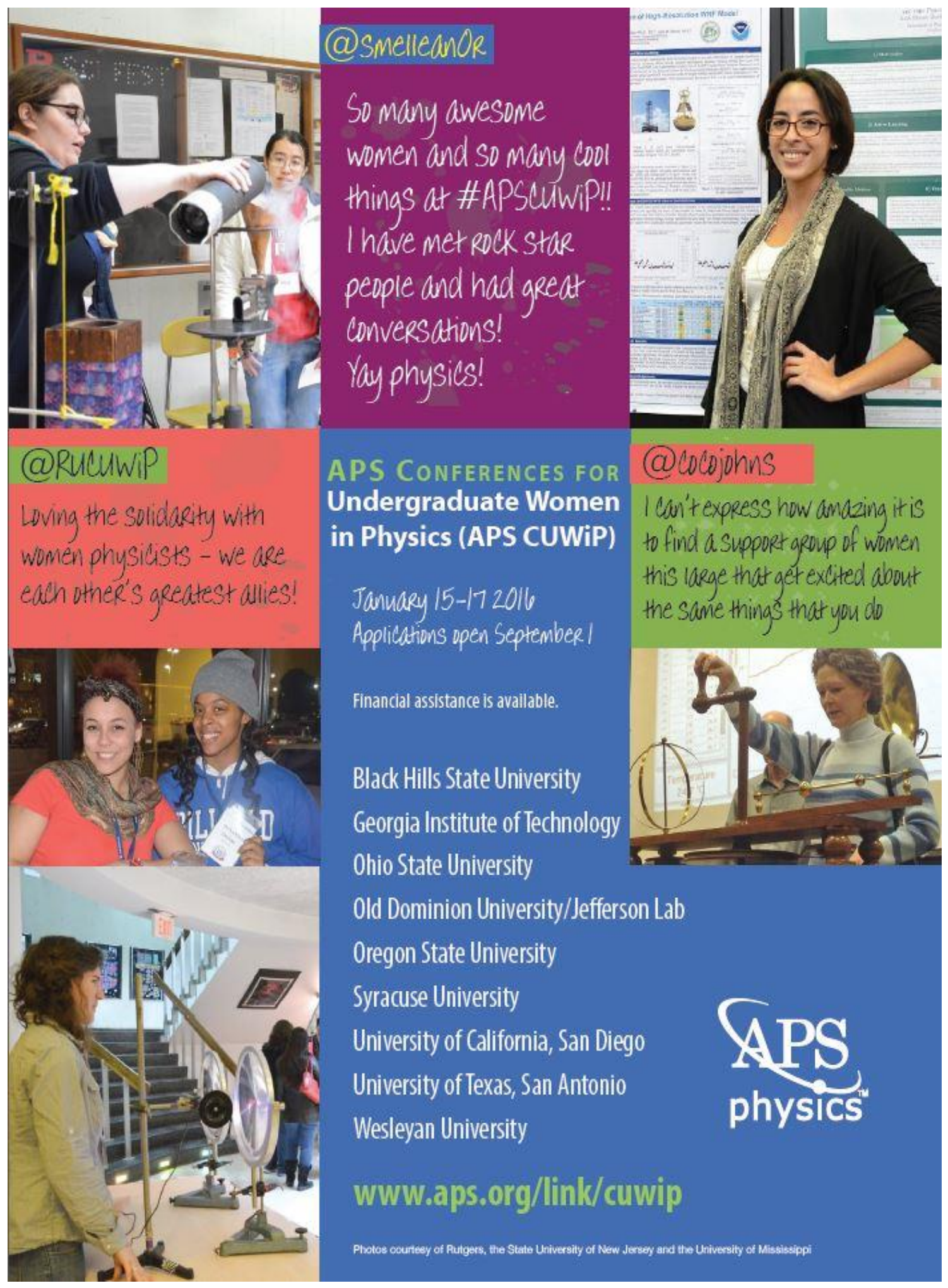


Appendix C: 2015-2016 CUWiP Survey

\section{CUWiP:}

\section{Conferences for Undergraduate Women in Physics Registration Survey}

Researchers at the American Physical Society and Florida International University are interested in your career plans, attitudes, and prior experiences with respect to physics. By filling out this questionnaire you will help us find ways to improve undergraduate physics education and the CUWiP experience for future students. You will be contacted after the CUWiP and will be asked

to take an additional survey. Make your best estimate for each item and answer as many questions as possible. Thank you for your help.

- You must be 18 years or older to participate.

- The survey will take approximately 15 minutes to complete.

- Participation is voluntary. You may withdraw at any time.

- Your participation has no adverse effects.

- If you have any questions or concerns, please contact Renee Michelle Goertzen at the American Physical Society at 301-209-3299 or goertzen@aps.org.

- You may contact the University of Maryland Institutional Review Board at 301-405-4212 or irb@umd.edu if you have any questions regarding your rights as a research participant.

Confidentiality: Your name and any other identifying information will NOT be included in any reports, published or unpublished, arising from this study. 


\section{ABOUT YOUR CAREER PLANS:}

1. To what extent would you consider pursuing the following careers with a background in physics:

\begin{tabular}{|c|c|c|c|c|c|}
\hline & Not at all 0 & 1 & 2 & 3 & 4 Very much so \\
\hline Professor & 0 & 0 & 0 & 0 & 0 \\
\hline Industry scientist & 0 & $\mathrm{O}$ & $\mathrm{O}$ & $\mathrm{O}$ & $\mathrm{O}$ \\
\hline Research/lab scientist & 0 & $\mathrm{O}$ & $\mathrm{O}$ & $\mathrm{O}$ & 0 \\
\hline Engineer & 0 & $\mathrm{O}$ & 0 & 0 & 0 \\
\hline High school teacher & o & $\mathrm{O}$ & 0 & O & 0 \\
\hline Professional (e.g. business, finance, law, medicine) & o & $\mathrm{O}$ & 0 & 0 & 0 \\
\hline Other related profession (e.g. science writer, policy) & o & $\mathrm{O}$ & 0 & $\mathrm{O}$ & 0 \\
\hline
\end{tabular}

2. Do you plan to attend graduate school in physics?

Not at all $0 \begin{array}{lllll} & 1 & 2 & 3 & 4\end{array}$ Very much so

3. Do you intend to complete a bachelors degree in physics?

Not at all $0 \begin{array}{lllll} & 1 & 2 & 3 & 4\end{array}$ Very much so

\section{Do you believe that physics careers allow the fulfillment of the following goals?}

\begin{tabular}{|c|c|c|c|c|}
\hline & Not at all 01 & 2 & 3 & 4 Very much so \\
\hline Serving community & OC & 0 & 0 & 0 \\
\hline Working with people & $\mathrm{OC}$ & $\mathrm{O}$ & $\mathrm{O}$ & $\mathrm{O}$ \\
\hline Altruism (selflessness, self-sacrifice) & 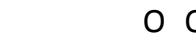 & 0 & 0 & 0 \\
\hline Helping others & O c & 0 & 0 & 0 \\
\hline Connecting with others & 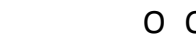 & $\mathrm{O}$ & 0 & 0 \\
\hline Serving humanity & 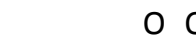 & 0 & $\mathrm{O}$ & 0 \\
\hline Attending to others & $\mathrm{OC}$ & 0 & $\mathrm{O}$ & 0 \\
\hline Caring for others & OC & 0 & $\mathrm{O}$ & 0 \\
\hline Spirituality & $\mathrm{OC}$ & $\mathrm{O}$ & $\mathrm{O}$ & 0 \\
\hline Intimacy & 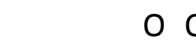 & 0 & $\mathrm{O}$ & 0 \\
\hline Power & $\mathrm{OC}$ & $\mathrm{O}$ & 0 & $\mathrm{O}$ \\
\hline Recognition & $\mathrm{OC}$ & 0 & $\mathrm{o}$ & $\mathrm{O}$ \\
\hline Achievement & O C & 0 & 0 & 0 \\
\hline Status & 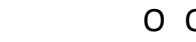 & 0 & 0 & 0 \\
\hline Focus on the self & 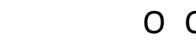 & $\mathrm{O}$ & 0 & 0 \\
\hline Success & 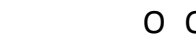 & 0 & 0 & 0 \\
\hline Financial reward & 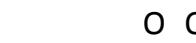 & 0 & $\mathrm{O}$ & 0 \\
\hline Self-direction & $\mathrm{OC}$ & $\mathrm{O}$ & 0 & 0 \\
\hline Mastery (command of knowledge/skills) & $\mathrm{OC}$ & $\mathrm{O}$ & $\mathrm{O}$ & 0 \\
\hline Self-promotion & $\mathrm{OC}$ & $\mathrm{O}$ & $\mathrm{O}$ & 0 \\
\hline Independence & $\mathrm{OC}$ & $\mathrm{O}$ & $\mathrm{O}$ & 0 \\
\hline Individualism & $\mathrm{OC}$ & 0 & $\mathrm{O}$ & 0 \\
\hline Demonstrating skill & $\mathrm{OC}$ & 0 & $\mathrm{o}$ & $\mathrm{O}$ \\
\hline Competition & Oc & 0 & 0 & $\mathrm{O}$ \\
\hline
\end{tabular}




\section{ABOUT YOUR PHYSICS ATTITUDES AND EXPERIENCES:}

For Questions 5 and 6, think about yourself as compared to an exemplary physics student you know/knew.

5. If applicable, to what extent do you believe the following people...

\begin{tabular}{|c|c|c|c|c|c|}
\hline & Not at all 0 & 1 & 2 & 3 & 4 Very much so \\
\hline \multicolumn{6}{|c|}{...see you as an exemplary physics student? } \\
\hline Yourself & $\mathrm{O}$ & 0 & $\mathrm{O}$ & 0 & $\mathrm{O}$ \\
\hline Your HS physics teacher(s) & $\mathrm{O}$ & 0 & $\mathrm{O}$ & $\mathrm{O}$ & $\mathrm{O}$ \\
\hline Other physics undergraduates & $\mathrm{O}$ & 0 & $\mathrm{O}$ & $\mathrm{O}$ & $\mathrm{O}$ \\
\hline Your physics professors/faculty & $\mathrm{O}$ & $\mathrm{O}$ & $\mathrm{O}$ & $\mathrm{O}$ & $\mathrm{O}$ \\
\hline \multicolumn{6}{|c|}{$\begin{array}{l}\text {...see another physics student you know as an exemplary } \\
\text { physics student? }\end{array}$} \\
\hline Yourself & $\mathrm{O}$ & 0 & $\mathrm{O}$ & $\mathrm{O}$ & $\mathrm{O}$ \\
\hline Your HS physics teacher(s) & $\mathrm{O}$ & 0 & $\mathrm{O}$ & $\mathrm{O}$ & $\mathrm{O}$ \\
\hline Other physics undergraduates & $\mathrm{O}$ & 0 & $\mathrm{O}$ & $\mathrm{O}$ & $\mathrm{O}$ \\
\hline Your physics professors/faculty & $\mathrm{O}$ & 0 & $\mathrm{O}$ & $\mathrm{O}$ & $\mathrm{O}$ \\
\hline
\end{tabular}

6. To what extent do you believe the following people...

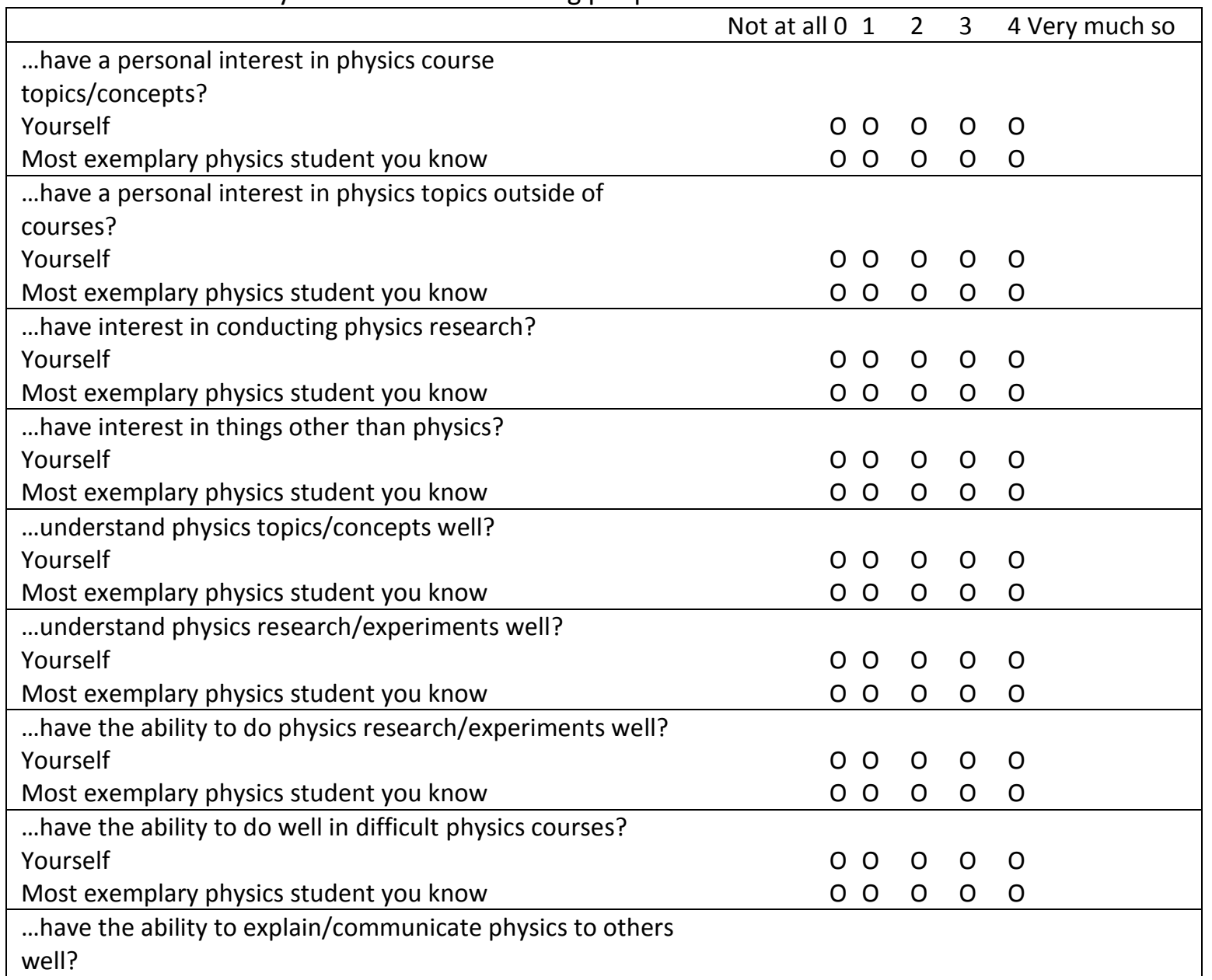


Yourself

Most exemplary physics student you know

$\begin{array}{lllll}0 & 0 & 0 & 0 & 0\end{array}$

$\begin{array}{lllll}0 & 0 & 0 & 0 & 0\end{array}$

7. To what extent do you believe your successes are due to...

\begin{tabular}{|c|c|c|c|c|c|}
\hline & Not at all 0 & 1 & 2 & 3 & 4 Very much so \\
\hline ...your ability & $\mathrm{O}$ & $\mathrm{O}$ & $\mathrm{O}$ & $\mathrm{O}$ & $\mathrm{O}$ \\
\hline ...your hard work & $\mathrm{O}$ & $\mathrm{O}$ & $\mathrm{O}$ & $\mathrm{O}$ & $\mathrm{O}$ \\
\hline ...good luck & $\mathrm{O}$ & $\mathrm{O}$ & $\mathrm{O}$ & $\mathrm{O}$ & $\mathrm{O}$ \\
\hline ...others' perceptions of you & $\mathrm{O}$ & O & 0 & $\mathrm{O}$ & 0 \\
\hline ...willingness to take advantage of opportunities & $\mathrm{O}$ & $\mathrm{O}$ & $\mathrm{O}$ & $\mathrm{O}$ & $\mathrm{O}$ \\
\hline ...proactively seeking out opportunities & $\mathrm{O}$ & 0 & 0 & 0 & 0 \\
\hline
\end{tabular}

8. With respect to a physics community, to what extent do you...

\begin{tabular}{|c|c|c|c|c|}
\hline & $\begin{array}{r}\text { Not at all } 1 \\
0\end{array}$ & 2 & 3 & 4 Very much so \\
\hline ...see yourself as a physicist & $\mathrm{OC}$ & $\mathrm{O}$ & $\mathrm{O}$ & 0 \\
\hline ...feel like you are part of the community & 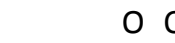 & 0 & 0 & 0 \\
\hline ...feel different from others in the community & 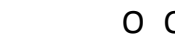 & $\mathrm{O}$ & 0 & 0 \\
\hline ...feel valued and respected & 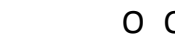 & 0 & $\mathrm{O}$ & $\mathrm{O}$ \\
\hline ...feel alone or isolated & O C & $\mathrm{O}$ & 0 & 0 \\
\hline ...feel you can share your thoughts/ideas & O C & $\mathrm{O}$ & $\mathrm{O}$ & 0 \\
\hline ...feel you can be heard & O C & $\mathrm{O}$ & $\mathrm{O}$ & 0 \\
\hline ...feel inadequate as a member & $\mathrm{OC}$ & 0 & $\mathrm{O}$ & 0 \\
\hline $\begin{array}{l}\text {...feel that others (students, faculty, etc.) are } \\
\text { accessible/available to help you }\end{array}$ & O C & $\mathrm{O}$ & $\mathrm{O}$ & 0 \\
\hline ...feel you can help others & $\mathrm{OC}$ & 0 & $\mathrm{O}$ & 0 \\
\hline
\end{tabular}

9. Have you previously attended any of the following types of physics conferences/events? Mark all that apply

O APS regional meeting O APS national meeting O Undergraduate research conference O Research-specific conference O Previous CUWiP O Other

10. Is there a Women in Physics (WiP) group at your university/institution?

O No O Yes

11. What level of involvement, if any, have you had as part of a Women in Physics (WiP) group at your university/institution? Mark all that apply

O No involvement O Attended meetings/events O Organized meetings/events

O Started a group O Encouraged others to join O Shared CUWiP

experiences/lessons

12. Other than representation (i.e. numbers participating), do you feel that there are serious gender issues in physics?

Not at all $0 \begin{array}{lllll}0 & 2 & 3 & 4 & \text { Very much so }\end{array}$

13. Do you believe there should be special events specifically for women in physics?

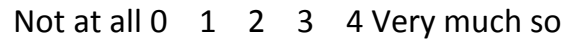




\section{ABOUT YOUR ACADEMIC BACKGROUND}

14. What year are you in college?

O First year O Second year O Third year O Fourth year O Fifth year O Graduate Student O Faculty

15. Which of the following physics courses (or equivalent) have you taken and completed in college? Mark all that apply

O Intro Physics I O Classical Mechanics (not intro) O Electromagnetism I O Quantum Mechanics I O Intro Physics II O Thermodynamics (Stat. Mech.) O Electromagnetism II O Quantum Mechanics II O Modern Physics

16. What is your approximate average GPA ( $4=A, 3=B, 2=C, 1=D, 0=F)$ in these physics courses please enter a number (decimals allowed)?

17. Which of the following mathematics courses (or equivalent) have you taken and completed in college? Mark all that apply

O Pre-calculus O Calculus I O Calculus II O Calculus III O Differential Equations O Linear Algebra

18. What is your approximate average GPA ( $4=A, 3=B, 2=C, 1=D, 0=F)$ in these mathematics courses - please enter a number (decimals allowed)?

\section{ABOUT YOU}

19. How important are each of the following kinds of goals to you personally?

\begin{tabular}{|c|c|c|c|c|}
\hline & Not at all 01 & 2 & 3 & 4 Very much so \\
\hline Serving community & 00 & $\mathrm{O}$ & $\mathrm{O}$ & $\mathrm{O}$ \\
\hline Working with people & 00 & $\mathrm{O}$ & $\mathrm{O}$ & $\mathrm{O}$ \\
\hline Altruism (selflessness, self-sacrifice) & 00 & $\mathrm{O}$ & $\mathrm{O}$ & $\mathrm{O}$ \\
\hline Helping others & 00 & $\mathrm{O}$ & $\mathrm{O}$ & $\mathrm{O}$ \\
\hline Connecting with others & 00 & $\mathrm{O}$ & $\mathrm{O}$ & $\mathrm{O}$ \\
\hline Serving humanity & 00 & $\mathrm{O}$ & $\mathrm{O}$ & $\mathrm{O}$ \\
\hline Attending to others & 00 & $\mathrm{O}$ & $\mathrm{O}$ & $\mathrm{O}$ \\
\hline Caring for others & $\mathrm{O} O$ & $\mathrm{O}$ & $\mathrm{O}$ & $\mathrm{O}$ \\
\hline Spirituality & 00 & $\mathrm{O}$ & $\mathrm{O}$ & $\mathrm{O}$ \\
\hline Intimacy & 00 & $\mathrm{O}$ & $\mathrm{O}$ & $\mathrm{O}$ \\
\hline Power & 00 & $\mathrm{O}$ & $\mathrm{O}$ & $\mathrm{O}$ \\
\hline Recognition & 00 & $\mathrm{O}$ & $\mathrm{O}$ & $\mathrm{O}$ \\
\hline Achievement & 00 & $\mathrm{O}$ & $\mathrm{O}$ & $\mathrm{O}$ \\
\hline Status & 00 & $\mathrm{O}$ & $\mathrm{O}$ & $\mathrm{O}$ \\
\hline Focus on the self & 00 & $\mathrm{O}$ & $\mathrm{O}$ & $\mathrm{O}$ \\
\hline Success & 00 & $\mathrm{O}$ & $\mathrm{O}$ & $\mathrm{O}$ \\
\hline Financial reward & 00 & $\mathrm{O}$ & $\mathrm{O}$ & $\mathrm{O}$ \\
\hline Self-direction & 00 & $\mathrm{O}$ & $\mathrm{O}$ & $\mathrm{O}$ \\
\hline Mastery (command of knowledge/skills) & 00 & $\mathrm{O}$ & $\mathrm{O}$ & $\mathrm{O}$ \\
\hline Self-promotion & 00 & $\mathrm{O}$ & $\mathrm{O}$ & $\mathrm{O}$ \\
\hline Independence & 00 & $\mathrm{O}$ & $\mathrm{O}$ & $\mathrm{O}$ \\
\hline
\end{tabular}


Individualism

Demonstrating skill

Competition $\begin{array}{lllll}0 & 0 & 0 & 0 & 0\end{array}$

$\begin{array}{lllll}0 & 0 & 0 & 0 & 0\end{array}$

$\begin{array}{lllll}0 & 0 & 0 & 0 & 0\end{array}$

20. Are you Female or Male?

O Female O Male O Other:

21. Are you Hispanic or Latino(a)?

O No OYes

22. With which racial group(s) do you identify? (For multi-racial, mark all that apply)

O Black O White O Asian O Native American or Alaskan Native O Native Hawaiian or Pacific Islander

O Other:

23. What is the highest level of education for your parents/guardians?

\begin{tabular}{|c|c|c|c|c|c|c|}
\hline & $\begin{array}{l}\text { Less than High } \\
\text { School Diploma }\end{array}$ & $\begin{array}{c}\text { High School } \\
\text { Diploma/GED }\end{array}$ & $\begin{array}{c}\text { Some College/ } \\
\text { Associate } \\
\text { Degree }\end{array}$ & $\begin{array}{l}\text { Bachelor's } \\
\text { Degree }\end{array}$ & $\begin{array}{l}\text { Master's } \\
\text { Degree or } \\
\text { higher }\end{array}$ & $\begin{array}{c}\text { Not } \\
\text { applicable }\end{array}$ \\
\hline \begin{tabular}{|c|} 
Male \\
parent/guardian
\end{tabular} & 0 & 0 & 0 & 0 & 0 & 0 \\
\hline $\begin{array}{c}\text { Female } \\
\text { parent/guardian }\end{array}$ & $\mathrm{O}$ & 0 & 0 & 0 & 0 & 0 \\
\hline
\end{tabular}

24. What is your home zip code?

25. Describe equity issues you believe exist in physics and explain why you think so. If you believe no equity issues exist, please write "none" and explain why you think so.

26. Listed below are the names of other participants attending the CUWiP conference for which you are registering. Please review this list of participants and place a checkmark by the name of any participants with whom you have previously interacted.

[Name] [checkbox]

[Name] [checkbox]

[Name] [checkbox]

[Etc.] 
[After a name is checked]

\begin{tabular}{|c|c|c|c|c|}
\hline $\begin{array}{l}\text { Know } \\
\text { personally }\end{array}$ & $\begin{array}{l}\text { Have } \\
\text { communicated } \\
\text { by email or }\end{array}$ & $\begin{array}{l}\text { Through Social } \\
\text { media (Facebook, } \\
\text { Twitter, Linked } \\
\text { In, Google+, etc.) }\end{array}$ & $\begin{array}{l}\text { Have spoken at } \\
\text { a professional } \\
\text { conference }\end{array}$ & $\begin{array}{l}\text { Met at } \\
\text { previous } \\
\text { CUWiP }\end{array}$ \\
\hline
\end{tabular}

How do you know

this person?

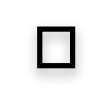

Above the "Submit" button at the bottom of the page:

Completion of this survey indicates that you are at least 18 years of age, that you have read the consent statement at the top of the page, and that you voluntarily agree to participate. If you agree, please complete the survey by clicking "Submit". 
Appendix D: Qualitative Strand Interview Protocol

1. Introduction/building rapport

a. Tell me about yourself

i. Where are you from?

ii. What school do you attend?

iii. What year are you?

iv. What is your major?

v. What do you like to do in your free time?

b. Why did you choose your major?

c. What is it like to be a (physics, engineering, etc.) major?

d. Do people ever make comments when you tell them you are a (physics, engineering, etc.) major? If so, what do they say?

i. Do these comments ever make you think about your major? In what way?

2. Explore the conceptual framework through some of the following questions depending on what is shared during the intro:

\section{Gender Roles}

e. Are you working on a research project currently, or taking a lab? Or focused mainly on coursework?

f. Are you involved in a study group, or a research team? How did you get connected with that team?

g. Reflect on the people you work with in your courses/lab/research, who do you work with? (follow up if clarification is requested: Are they men, women, how old are they, undergrads, etc.?)

h. Describe your teammates and who is working on each set of responsibilities along the way.

i. Or: Walk me through a lab, research, or study team meeting. Who is working on what? How do you describe the dynamics of the group in the meeting? Outside of the meeting?

i. What do you work on in these/this team setting(s)?

j. What do others work on?

k. Think of a time when you were primarily interacting with male students in your physics/engineering classes, and then of a time when you were primarily interacting with female students, how are they similar or different?

1. (if they discuss gendered roles or differences) Do you perceive any difference in the roles men and women play in these groups?

m. OR: Do you ever think of these roles as gendered (i.e., some roles are better suited for the male team members or the female team members)? 
n. Why do you think we don't see women in physics at higher rates? If follow up is appropriate or helpful: Why do we see more women in biology, for example?

o. Do you consider your major to be male-dominated? Why/why not?

p. Do you consider your major to be better suited for male students? Why? Why not?

q. Do you think about yourself as a female in a male-dominated field?

i. If no, why not?

ii. If yes, what do you think about? What example(s) represent the way you think about this?

r. Did you consider any other majors or careers?

s. Do you think that physics is perceived as being able to help people? Do you perceive it that way? Why? Why not?

t. Do you think that physics is perceived as a field that is powerful?

\section{"Ideal" Science Student}

a. Who are the "good" or "successful" students in your STEM classes? How or why do you know this? Describe this/these student(s). (Or, more colloquially: Who are the awesome students in your classes and why/how do you know?)

a. What is it about this person that makes them the best student?

b. Are you a "good" or "successful" student in your STEM classes? Why/Why not?

a. Or, do you consider yourself one of the best students in the class? Why/Why not?

c. How does the professor(s)/instructor(s) treat the students in the class who are successful compared to the students who are not successful?

d. When was the first time you learned about the type of person who is most likely to be good at physics/engineering?

i. Why did you think that? What was the context of the example you've shared?

\section{Physics Identity}

u. Can you share an example of a time when you felt really interested in your major?

i. What were the circumstances?

v. Can you share an example of a time when you felt competent in your major?

i. What were the circumstances?

w. Can you share an example of a time when you were proud of your performance in your major?

i. What were the circumstances?

x. Can you share an example of a time when you felt recognized in your major?

i. What were the circumstances? 
ii. When did you feel really good about yourself in physics?

3. How do they articulate their plans to persist in their chosen field?

y. Do you like your major?

i. Why? Or, why not?

z. Are you thinking about keeping your major until graduation?

i. Why? Or, why not?

aa. If they've already graduated: What's next?

i. Are you planning to attend graduate school for the same subject you're currently studying?

ii. Why? Or, why not?

bb. What's your dream job?

cc. What external factors, if any, influence your decision to keep/change your major before you graduate?

dd. What internal factors, if any, influence your decision to keep/change your major before you graduate?

ee. And, are there any identity factors they privilege when making these plans (e.g., race, ethnicity, nationality, sexual orientation, ability, religious affiliation, science identity, etc.)?

\section{Generalized Perspectives}

ff. What are the types of things that people have said to you along the way that confirmed that you wanted to be a physics/engineering major?

gg. What are the types of things that people have said to you along the way that gave you second thoughts?

4. Final Question: Are there any other examples, stories, or thoughts you'd like to share before we wrap-up? 
Appendix E: Complete List of Codes

\begin{tabular}{|c|c|c|}
\hline \multicolumn{2}{|c|}{ Code System } & $\begin{array}{c}\text { Code } \\
\text { Frequencies }\end{array}$ \\
\hline \multicolumn{2}{|c|}{ Code System } & 2618 \\
\hline \multicolumn{2}{|c|}{ Persistence } & 41 \\
\hline & engineering plans & 9 \\
\hline & Influencing factors for plans to persist & 88 \\
\hline & Career plans & 52 \\
\hline & Graduate School & 52 \\
\hline & Bachelors degree persistence (actual/plans) & 18 \\
\hline \multicolumn{2}{|c|}{ Intrinsic Agentic gender roles/goals } & 2 \\
\hline & self-direction (IA) & 51 \\
\hline & independence (IA) & 55 \\
\hline & individualism (IA) & 60 \\
\hline & demonstrating skill (IA) & 46 \\
\hline \multicolumn{2}{|c|}{ Extrinsic Agentic gender roles/goals } & 5 \\
\hline & power (EA) & 70 \\
\hline & recognition $(\mathrm{EA})$ & 24 \\
\hline & status (EA) & 52 \\
\hline & success $(\mathrm{EA})$ & 38 \\
\hline & financial reward (EA) & 14 \\
\hline & self-promotion (EA) & 50 \\
\hline \multicolumn{2}{|c|}{ Communal gender roles/goals } & 12 \\
\hline & serving community & 10 \\
\hline & working with people & 97 \\
\hline & Altruism (selflessness, self-sacrifice) & 11 \\
\hline & helping others & 63 \\
\hline & connecting with others & 97 \\
\hline & serving humanity & 17 \\
\hline & attending to others & 39 \\
\hline & caring for others & 23 \\
\hline \multicolumn{2}{|c|}{ Other "gendered" reflections } & 123 \\
\hline & "Chilly Climate" & 81 \\
\hline & Times Change/Generation Diff and Gender & 26 \\
\hline \multicolumn{2}{|c|}{ Physics/Science Identity } & 10 \\
\hline & Recognition (identity) & 87 \\
\hline & Interest (identity) & 117 \\
\hline & Competence (identity) & 108 \\
\hline
\end{tabular}




\begin{tabular}{|c|c|}
\hline Performance (identity) & 84 \\
\hline "Ideal" Science Student/Scientist & 102 \\
\hline Recognition ("Ideal") & 24 \\
\hline Interest ("Ideal") & 19 \\
\hline Competence ("Ideal") & 55 \\
\hline Performance ("Ideal") & 41 \\
\hline Other/Misc & 0 \\
\hline Omitting phys/math major with others & 5 \\
\hline Leadership & 39 \\
\hline Imposter Syndrome & 48 \\
\hline GRIT/resilience & 52 \\
\hline Prejudice/discrimination & 93 \\
\hline Physics Culture & 98 \\
\hline Diversity in STEM & 102 \\
\hline Support/Lack of Support & 151 \\
\hline CUWiP Reflections & 11 \\
\hline Mentoring & 34 \\
\hline Harassment & 12 \\
\hline
\end{tabular}


Appendix F: Email Invitation to Participate in the Qualitative Phase of the Study

Dear ,

My name is Bronwen Bares Pelaez, and I am a researcher at Florida International University in Miami, Florida. For a research project we're conducting in association with CUWiP, we're interested in examining women's attitudes and experiences in physics. Our hope is that this study will add to the field of research focused on closing the gender gap persistent in some science, technology, engineering, and math (STEM) fields.

Because of your participation in CUWiP, we would like to invite you to participate in this study regarding your experiences in your major. Participation will consist of an interview over the phone with me, which will require about an hour of your time. The questions will be open-ended, and will be digitally recorded. The recording of the interview will be transcribed and shared with you about one or two weeks after the interview has been completed. You will have the opportunity to review your interview transcripts for accuracy, and submit any corrections if you choose.

All participant information will be kept confidential. By participating in this study, there are no risks beyond that of an individual's daily routine, and there are no specific benefits, although the insights provided by your participation could help women in physics in the future. Additionally, as a thank you for your time we will send you a \$20 Starbucks gift card.

If you would like to participate, please respond to this email as soon as possible to set up a time for the phone interview. I thank you in advance for your willingness to participate in this research study.

Bronwen Bares Pelaez

Doctoral Candidate: FIU Higher Education Administration

Director, Women's Center

Division of Student Affairs

Florida International University

baresb@fiu.edu, 305-348-1506

If you have any concerns regarding this study that you would like to share beyond the researcher listed above, please contact the Principle Investigator, Dr. Zahra Hazari, zhazari@fiu.edu (FIU IRB Approval \#: IRB-16-0249). 
Appendix G: Email Follow up to Qualitative Participants

Dear ,

I hope that this email finds you well! Attached you will find the transcript of our interview a couple weeks ago. As I mentioned when we talked, you are welcome to look it over and send any feedback, clarifications, or additions, however this is not required as part of your participation in the study.

Also, your Starbucks cards are in the mail and should be arriving any day now. Please send me a quick confirmation email when you receive them for my records.

Thank you again for your time and insights, I really appreciate your participation in this study!

Sincerely,

Bronwen

$\overline{\text { Bronwen Bares Pelaez }}$

Doctoral Candidate: Higher Education Administration

Florida International University

School of Education and Human Development

baresb@fiu.edu

305-348-1506 
Appendix H: MAXQDA Display of the Code System Including Subcodes

\begin{tabular}{|c|c|c|}
\hline Code System & 용 & $5 \times$ \\
\hline 咍 & $D$ & \# \\
\hline$\triangle$ Code System & & 2618 \\
\hline$\triangle$ Persistence & 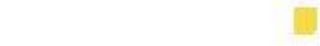 & 41 \\
\hline engineering plans & & 9 \\
\hline Influencing factors for plans to persist & & 88 \\
\hline - Career plans & & 52 \\
\hline Graduate School & & 52 \\
\hline Bachelors degree persistence (actual/plans) & & 18 \\
\hline$\Delta$ Intrinsic Agentic gender roles/goals & in & 2 \\
\hline self-direction (IA) & & 51 \\
\hline independence (IA) & & 55 \\
\hline individualism (IA) & & 60 \\
\hline demonstrating skill (IA) & & 46 \\
\hline$\Delta \quad$ Extrinsic Agentic gender roles/goals & W & 5 \\
\hline power (EA) & & 70 \\
\hline recognition (EA) & & 24 \\
\hline status (EA) & & 52 \\
\hline success (EA) & & 38 \\
\hline financial reward (EA) & & 14 \\
\hline self-promotion (EA) & & 50 \\
\hline$\triangleright \bullet$ Communal gender roles/goals & in & 369 \\
\hline$D$ Other "gendered" reflections & & 230 \\
\hline$D$ Physics/Science Identity & in & 406 \\
\hline$\triangleright \sigma_{-}$"Tdeal" Science Student/Scientist & 勧 & 241 \\
\hline$\triangleright \quad$ Other/Misc & & 645 \\
\hline त Sets & & 0 \\
\hline
\end{tabular}

In this view, the codes "Persistence," "Intrinsic Agentic Gender Roles/Goals," and "Extrinsic Agentic Gender Roles/Goals" are expanded to display their subcodes. 


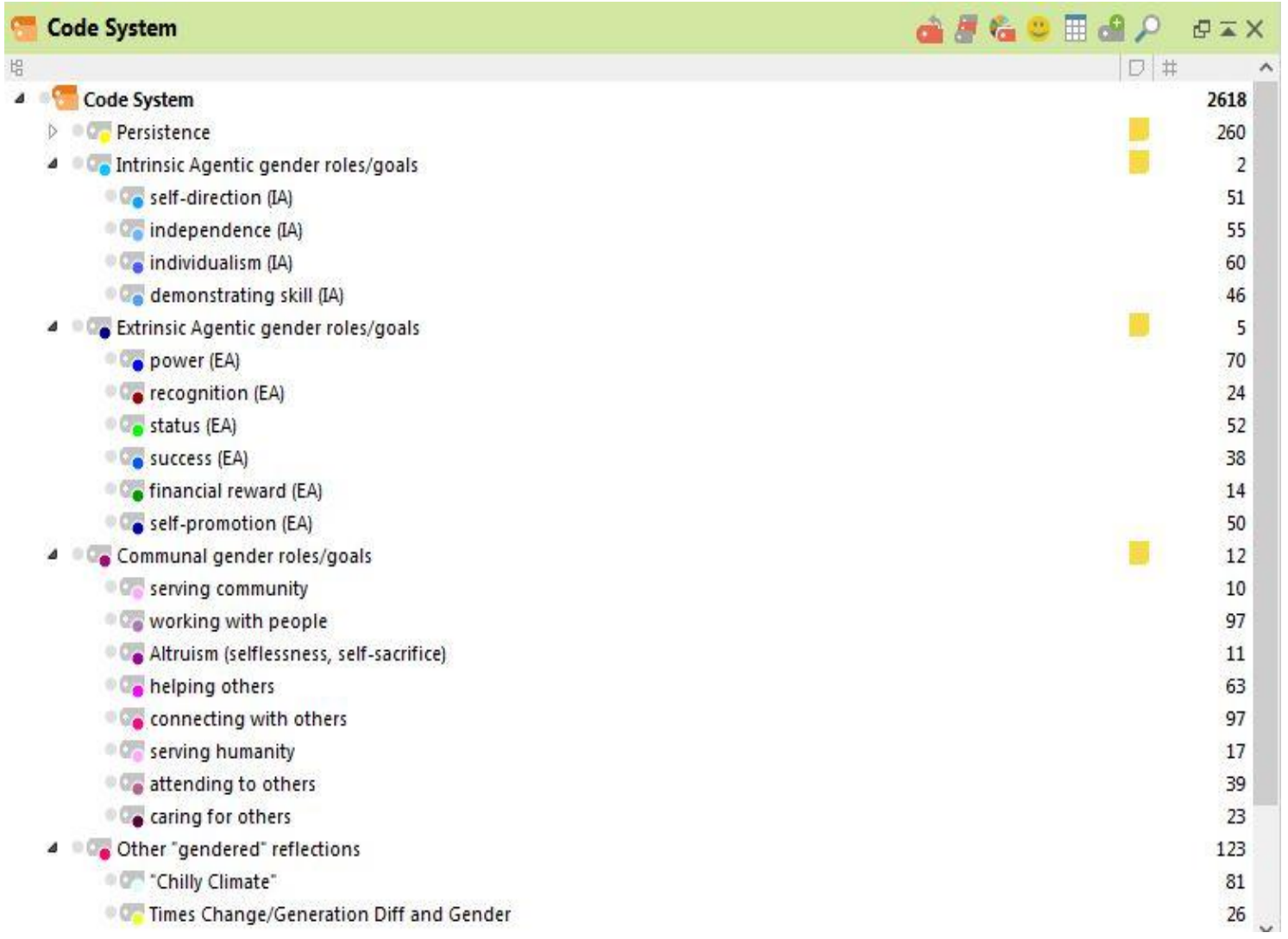

In this view, all gender-related codes are expanded to display their subcodes (i.e., "Intrinsic Agentic Gender Roles/Goals," "Extrinsic Agentic Gender Roles/Goals," "Communal Gender Roles/Goals," and "Other 'Gendered' Reflections" are expanded to display their subcodes). 


\begin{tabular}{|c|c|c|c|}
\hline Co & de System & $\mathrm{C}^{+}$: & $\square=x$ \\
\hline 놈 & & $D \neq$ & \# $\quad$ A \\
\hline$\triangleright$ & Extrinsic Agentic gender roles/goals & 1 & 253 \\
\hline$\triangleright$ & Communal gender roles/goals & in & 369 \\
\hline D & Other "gendered" reflections & & 230 \\
\hline$\Delta$ & oghysics/Science Identity & [1] & 10 \\
\hline & Recognition (identity) & & 87 \\
\hline & Interest (identity) & & 117 \\
\hline & Competence (identity) & & 108 \\
\hline & Performance (identity) & & 84 \\
\hline$\Delta$ & $\sigma_{-}$'Tdeal" Science Student/Scientist & 国 & 102 \\
\hline & $\bullet$ Recognition ('Tdeal) & & 24 \\
\hline & Interest ('Tdeal') & & 19 \\
\hline & Competence ('Tdeal') & & 55 \\
\hline & Performance ('Tdeal') & & 41 \\
\hline$\Delta$ & Other/Misc & & 0 \\
\hline & Omitting phys/math major with others & & 5 \\
\hline & Leadership & II & 39 \\
\hline & - Imposter Syndrome & & 48 \\
\hline & GRT/resilience & 滀 & 52 \\
\hline & Physics Culture & & 98 \\
\hline & Diversity in STEM & & 102 \\
\hline & $\Delta \quad$ Support/Lack of Support & & 151 \\
\hline & CUWiP Reflections & & 11 \\
\hline & Mentoring & & 34 \\
\hline & Harassment & & 12 \\
\hline & Prejudice/discrimination & & 93 \\
\hline
\end{tabular}

In this view, the codes "Physics Identity," "'Ideal' Science Student/Scientist," and "Other/Misc" are expanded to display their subcodes. 
Appendix I: R Code Written to Conduct Quantitative Analysis

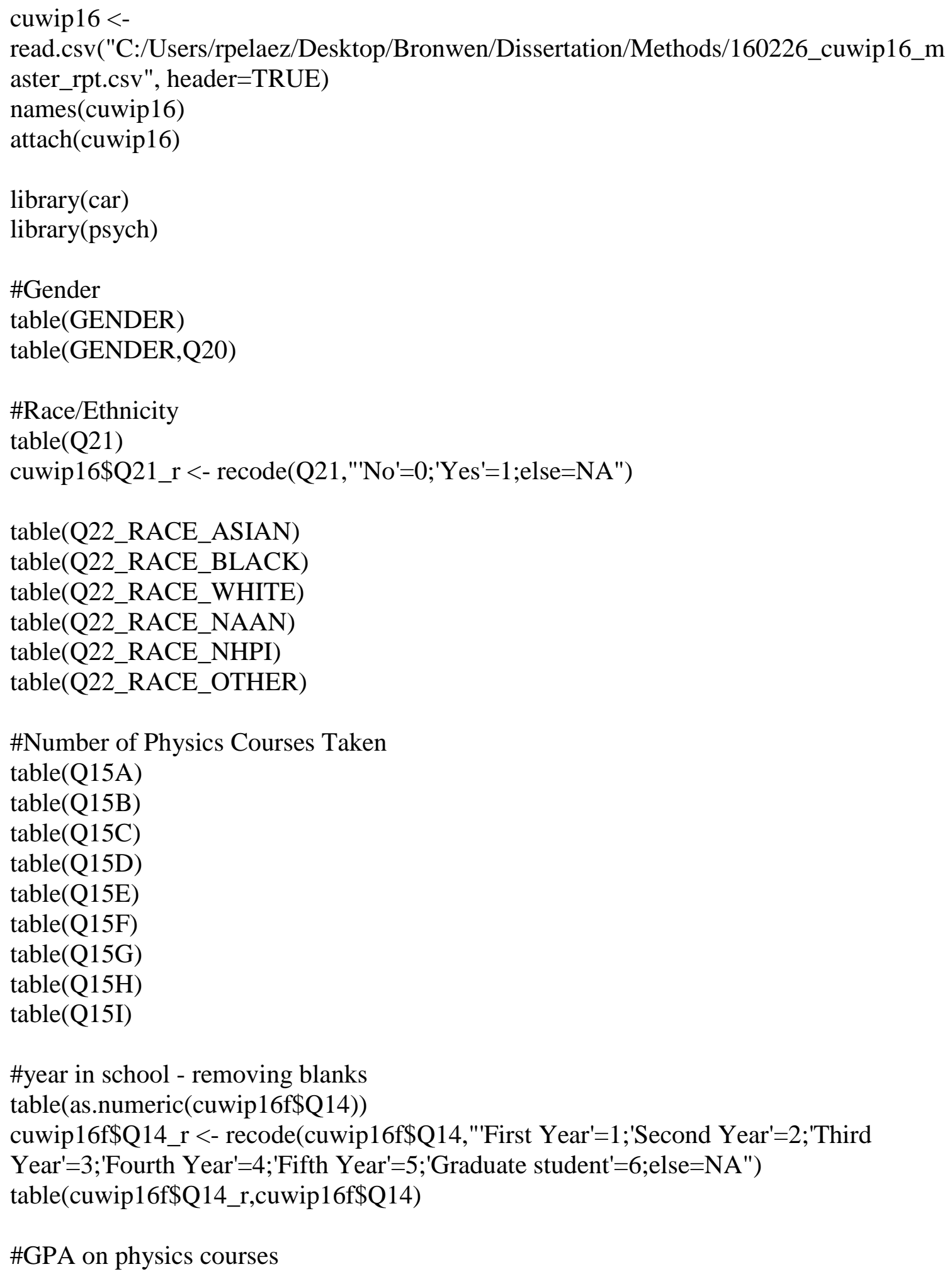


table(cuwip16f\$Q16)

cuwip16f\$Q16_r <- recode(cuwip16f\$Q16,"275=NA")

table(cuwip16f\$Q16_r,cuwip16f\$Q16)

table(cuwip16f\$Q16_r)

describe(cuwip16f\$Q16_r)

\#Create data frame - gender role congruity Q19

attach (cuwip16)

cuwipQ19 = as.data.frame $(\operatorname{cbind}(\mathrm{Q} 19 \mathrm{~A}, \mathrm{Q} 19 \mathrm{~B}, \mathrm{Q} 19 \mathrm{C}, \mathrm{Q} 19 \mathrm{D}, \mathrm{Q} 19 \mathrm{E}, \mathrm{Q} 19 \mathrm{~F}, \mathrm{Q} 19 \mathrm{G}$, Q19H, Q19I, Q19J, Q19K, Q19L, Q19M, Q19N, Q19O, Q19P, Q19Q, Q19R, Q19S, Q19T, Q19U, Q19V, Q19W, Q19X))

cuwipQ19 = na.omit (cuwipQ19)

\#Run Factor Analysis

print (factanal(cuwipQ19, 3, rotation="promax")\$loadings, sort=T, cutoff=0.4)

\#Revised factor analysis

cuwipQ19b = as.data.frame(cbind(Q19A, Q19B, Q19C, Q19D, Q19E, Q19F, Q19G, Q19H, Q19K, Q19L, Q19M, Q19N, Q19O, Q19P, Q19Q, Q19T, Q19X))

cuwipQ19b = na.omit (cuwipQ19b)

print (factanal(cuwipQ19b, 2, rotation="promax")\$loadings, sort=T, cutoff=0.4)

\#Chronbach's alpha overall (need to break into two and run)

alpha(cuwipQ19b)

\#3 factor gender role congruity

cuwipQ19c = as.data.frame (cbind(Q19A, Q19B, Q19C, Q19D, Q19E, Q19F, Q19G, Q19H, Q19K, Q19L, Q19N, Q19P, Q19Q, Q19R, Q19T, Q19U, Q19V, Q19W, Q19X)) cuwipQ19c = na.omit (cuwipQ19c)

print (factanal(cuwipQ19c, 3, rotation="promax")\$loadings, sort=T, cutoff=0.4)

\#Chronbach's alpha overall (alpha for each constuct below scree plot)

alpha(cuwipQ19c)

\#Determine Number of Factors (Scree Plot)

library(nFactors)

ev <- eigen(cor(cuwipQ19c))

ap <- parallel(subject=nrow(cuwipQ19c), var=ncol(cuwipQ19c),rep=100,cent=.05)

$\mathrm{nS}<-\mathrm{nScree}(\mathrm{x}=\mathrm{ev}$ \$values, aparallel=ap\$eigen $\$$ qevpea $)$

plotnScree $(\mathrm{nS})$

\#get packages and include alpha for both items 
\#Create Agentic and Communal Constructs

$\operatorname{attach}($ cuwipQ19c)

names(cuwipQ19c)

alpha(as.data.frame(cbind(Q19A,Q19B,Q19C,Q19D,Q19E,Q19F,Q19G,Q19H)))

alpha(as.data.frame(cbind(Q19K,Q19L,Q19N,Q19P,Q19Q,Q19T,Q19X)))

alpha(as.data.frame(cbind(Q19R,Q19U,Q19V,Q19W)))

$\operatorname{detach}($ cuwipQ4c)

\#Create Communal, Extrinsic Agentic, and Intrinsic Agentic Constructs

cuwip16\$Q19communal <-

(cuwip16\$Q19A+cuwip16\$Q19B+cuwip16\$Q19C+cuwip16\$Q19D+cuwip16\$Q19E+cu

wip16\$Q19F+cuwip16\$Q19G+cuwip16\$Q19H)/8

describe(cuwip16\$Q19communal)

cuwip16\$Q19agentic_extrinsic <-

(cuwip16\$Q19K+cuwip16\$Q19L+cuwip16\$Q19N+cuwip16\$Q19P+cuwip16\$Q19Q+cu

wip16\$Q4T+cuwip16\$Q4X)/7

describe(cuwip16\$Q19agentic_extrinsic)

cuwip16\$Q19agentic_intrinsic <-

(cuwip16\$Q19R+cuwip16\$Q19U+cuwip16\$Q19V+cuwip16\$Q19W)/4

describe(cuwip16\$Q19agentic_intrinsic)

\#Create data frame for factor analysis on the "ideal" scientist items

detach(cuwip16)

attach(cuwip16)

cuwipQ5 <-

as.data.frame(cbind(Q5A,Q5B,Q5C,Q5D,Q5E,Q5F,Q5G,Q5H,Q6A,Q6B,Q6C,Q6D,Q6E ,Q6F,Q6G,Q6H,Q6I,Q6J,Q6K,Q6L,Q6M,Q6N,Q6O,Q6P,Q6Q,Q6R))

cuwipQ5 <- na.omit(cuwipQ5, cuwipQ6)

\#Run Factor analysis for Identity and "Ideal" Science Student

print(factanal(cuwipQ5, 1, rotation="promax")\$loadings, sort=T, cutoff=0.4)

cuwipQ5 <-

as.data.frame(cbind(Q5A,Q5B,Q5C,Q5D,Q6A,Q6C,Q6E,Q6G,Q6I,Q6K,Q6M,Q6O,Q6Q

) )

cuwipQ5 <- na.omit(cuwipQ5, cuwipQ6)

$\operatorname{print}($ factanal(cuwipQ5, 3, rotation="promax")\$loadings, sort=T, cutoff=0.4) 
cuwipQ5 <-

as.data.frame(cbind(Q5E,Q5F,Q5G,Q5H,Q6B,Q6D,Q6F,Q6H,Q6J,Q6L,Q6N,Q6P,Q6R))

cuwipQ5 <- na.omit(cuwipQ5, cuwipQ6)

$\operatorname{print}($ factanal(cuwipQ5, 3, rotation="promax")\$loadings, sort=T, cutoff=0.4)

\#Factor analysis B - self_identity (removed Q5B, Q5G)

cuwipQ5 <-

as.data.frame(cbind(Q5A,Q5C,Q5D,Q6A,Q6C,Q6E,Q6I,Q6K,Q6M,Q6O,Q6Q))

cuwipQ5 <- na.omit(cuwipQ5, cuwipQ6)

$\operatorname{print}($ factanal(cuwipQ5, 3, rotation="promax")\$loadings, sort=T, cutoff=0.4)

\#Factor analysis C - self_identity (removed Q5B, Q6G, Q6I, Q6Q)

cuwipQ5 <- as.data.frame(cbind(Q5A,Q5C,Q5D,Q6A,Q6C,Q6E,Q6K,Q6M,Q6O))

cuwipQ5 <- na.omit(cuwipQ5, cuwipQ6)

$\operatorname{print}($ factanal(cuwipQ5, 3, rotation="promax")\$loadings, sort=T, cutoff=0.4)

\#Factor analysis D - self_identity with I added back in (removed Q5B, Q6G, Q6O, Q6Q)

cuwipQ5 <- as.data.frame(cbind(Q5A,Q5C,Q5D,Q6A,Q6C,Q6E,Q6K,Q6M,Q6I))

cuwipQ5 <- na.omit(cuwipQ5, cuwipQ6)

$\operatorname{print}($ factanal(cuwipQ5, 3, rotation="promax")\$loadings, sort=T, cutoff=0.4)

\#Factor analysis E - "ideal" science student

cuwipQ5 <-

as.data.frame(cbind(Q5E,Q5F,Q5G,Q5H,Q6B,Q6D,Q6F,Q6J,Q6L,Q6N,Q6P))

cuwipQ5 <- na.omit(cuwipQ5, cuwipQ6)

$\operatorname{print}($ factanal(cuwipQ5, 3, rotation="promax")\$loadings, sort=T, cutoff=0.4)

\#Factor analysis C - "ideal" science student (removed Q5F, Q6H, Q6P, Q6R)

cuwipQ5 <- as.data.frame(cbind(Q5E,Q5G,Q5H,Q6B,Q6D,Q6F,Q6J,Q6L,Q6N))

cuwipQ5 <- na.omit(cuwipQ5, cuwipQ6)

$\operatorname{print}($ factanal(cuwipQ5, 3, rotation="promax")\$loadings, sort=T, cutoff=0.4)

\#Chronbach's alpha for identity/self and then ideal science student (reliability)

alpha(as.data.frame(cbind(Q5A,Q5C,Q5D,Q6A,Q6C,Q6E,Q6K,Q6M,Q6I)))

alpha(as.data.frame(cbind(Q5E,Q5G,Q5H,Q6B,Q6D,Q6F,Q6J,Q6L,Q6N)))

\#Creating the constructs for "ideal" science student

cuwip16\$Q5_6idealsci<-(Q5E+Q5G+Q5H+Q6B+Q6D+Q6F+Q6J+Q6L+Q6N)/9 
table(cuwip16\$Q5_6idealsci)

describe(cuwip16\$2_6idealsci)

\#Creating the constructs for science identity

cuwip16\$Q5_6identity<-(Q5A+Q5C+Q5D+Q6A+Q6C+Q6E+Q6K+Q6M+Q6I)/9

table(cuwip16\$Q5_6identity)

describe(cuwip16\$Q5_6identity)

\#create data frame with only females

table(Q20)

table(GENDER)

table(GENDER,Q20)

cuwip16f $<$-subset(cuwip16,Q20=="Female")

\#Run linear regression for relationship between ideal and science identity (RQ1)

model<-lm(Q5_6identity $\sim$ Q5_6idealsci,data=cuwip16f)

summary(model)

plot(cuwip16f\$Q5_6idealsci,cuwip16f\$Q5_6identity)

abline(model)

library(lm.beta)

lm.beta(model)

cor.test(cuwip16f\$Q5_6idealsci,cuwip16f\$Q5_6identity)

\#Run linear regression for relationship between gender role congruity and science identity (RQ2)

model<-lm(Q5_6identity $\sim$ Q19communal,data=cuwip16f)

summary(model)

plot(cuwip16f\$Q19communal,cuwip16f\$Q5_6identity)

abline(model)

lm.beta(model)

cor.test(cuwip16f\$Q19communal,cuwip16f\$Q5_6identity)

model<-lm(Q5_6identity $\sim$ Q19agentic_extrinsic,data=cuwip16f)

summary(model)

plot(cuwip16f\$Q19agentic_extrinsic,cuwip16f\$Q5_6identity)

abline(model)

lm.beta(model)

cor.test(cuwip16f\$Q19agentic_extrinsic,cuwip16f\$Q5_6identity)

model<-lm(Q5_6identity $\sim$ Q19agentic_intrinsic,data=cuwip16f)

summary(model)

plot(cuwip16f\$Q19agentic_intrinsic,cuwip16f\$Q5_6identity)

abline(model)

lm.beta(model)

cor.test(cuwip16f\$Q19agentic_intrinsic,cuwip16f\$Q5_6identity) 
model<-

$\operatorname{lm}(\mathrm{Q} 5$ 6identity Q19communal+Q19agentic_extrinsic+Q19agentic_intrinsic,data=cuwi p16f)

summary(model)

lm.beta(model)

\#multicollinearity for model with more than two factors (less than 5 is good, less than 2 is great, less than 10 is sketchy)

vif(model)

cor.test(cuwip16f\$Q19agentic_extrinsic+cuwip16f\$Q19agentic_intrinsic+cuwip16f\$Q19 communal,cuwip16f\$Q5_6identity)

\#Create persistence outcome Q1ABCD (career intentions)

cuwip16f\$Q1ABCD<-

pmax(cuwip16f\$Q1A,cuwip16f\$Q1B,cuwip16f\$Q1C,cuwip16f\$Q1D)

describe(cuwip16f\$Q1ABCD)

\#Run linear regression for realtionship between gender role, identity, "ideal" sci and persistence as career intentions (RQ3)

model<-

$\operatorname{lm}(\mathrm{Q} 5$ 6identity Q19communal+Q19agentic_extrinsic+Q19agentic_intrinsic+Q5_6ideal sci,data=cuwip16f)

summary(model)

lm.beta(model)

plot(cuwip16f\$Q19communal,cuwip16f\$Q5_6identity)

abline(model)

$\operatorname{vif}($ model $)$

\#Tried GPA and Year in College, but not significant, so removed

\#linear regression for career plans and gender roles, identity, and "ideal" scientist

model<-

$\operatorname{lm}(\mathrm{Q} 1 \mathrm{ABCD}$ Q5_6identity+Q19communal+Q19agentic_extrinsic+Q19agentic_intrinsic +Q5_6idealsci,data=cuwip16f)

summary(model)

vif(model)

$\operatorname{lm}$. beta(model)

plot(cuwip16f\$Q19communal,cuwip16f\$Q1ABCD)

abline(model)

plot(cuwip16f\$Q5_6identity,cuwip16f\$Q1ABCD)

abline(model)

plot(cuwip16f\$Q19agentic_extrinsic,cuwip16f\$Q1ABCD)

abline(model)

plot(cuwip16f\$Q19agentic_intrinsic,cuwip16f\$Q1ABCD)

abline(model) 
plot(cuwip16f\$Q5_6idealsci,cuwip16f\$Q1ABCD)

abline(model)

model<-

$\operatorname{lm}(\mathrm{Q} 1 \mathrm{~A} \sim \mathrm{Q} 5$ _6identity+Q19communal+Q19agentic_extrinsic+Q19agentic_intrinsic+Q5 _6idealsci,data $=$ cuwip16f)

summary(model)

vif(model)

plot(cuwip16f\$Q5_6identity,cuwip16f\$Q1A)

abline(model)

model<-

$\operatorname{lm}(\mathrm{Q} 1 \mathrm{~B} \sim \mathrm{Q} 5$ 6identity+Q19communal+Q19agentic_extrinsic+Q19agentic_intrinsic+Q5_ 6idealsci+Q4agentic*Q19agentic_intrinsic,data=cuwip16f)

summary(model)

vif(model)

plot(cuwip16f\$Q19communal,cuwip16f\$Q5_6identity)

abline(model)

model<-

$\operatorname{lm}(\mathrm{Q} 1 \mathrm{C} \sim \mathrm{Q} 5$ 6identity+Q19communal+Q19agentic_extrinsic+Q19agentic_intrinsic+Q5_ 6idealsci+Q4agentic*Q19agentic_intrinsic,data=cuwip16f)

summary(model)

vif(model)

plot(cuwip16f\$Q19communal,cuwip16f\$Q5_6identity)

abline(model)

model<-

$\operatorname{lm}(\mathrm{Q} 1 \mathrm{D} \sim \mathrm{Q} 5$ _6identity+Q19communal+Q19agentic_extrinsic+Q19agentic_intrinsic+Q5

_6idealsci+Q4agentic*Q19agentic_intrinsic,data=cuwip16f)

summary(model)

vif(model)

plot(cuwip16f\$Q19communal,cuwip16f\$Q5_6identity)

abline(model)

\#Create persistence outcome Q2 (grad school intentions)

\#cuwip16f\$Q2

describe(cuwip16f\$Q2)

\#Linear Regression for Q2 (grad school intentions)

model<-

$\operatorname{lm}\left(\mathrm{Q} 2 \sim \mathrm{Q} 5 \_6 i d e n t i t y+Q 19\right.$ communal+Q19agentic_extrinsic+Q19agentic_intrinsic+Q5_6 idealsci,data=cuwip16f)

summary(model)

vif(model) 
$\operatorname{lm} . \operatorname{beta}(\operatorname{model})$

\#Create persistence outcome Q3 (bachelors completion intentions)

\#cuwip16f\$Q3

describe(cuwip16f\$Q3)

\#Linear Regression for Q3 (bachelor's completion intentions)

model<-

$\operatorname{lm}(\mathrm{Q} 3$ Q5_6identity+Q19communal+Q19agentic_extrinsic+Q19agentic_intrinsic+Q5_6 idealsci,data=cuwip16f)

summary(model)

vif(model)

plot (cuwip16f\$Q3, cuwip16f\$Q19communal)

abline (model)

lm.beta(model) 
VITA

\section{BRONWEN BARES PELAEZ}

1998-2002

2004-2006

2004-2006

2006-2009

2009-2011

2011-2014

2012

2014-present

2012-2017
Bachelor of Arts, Music Performance

with College Park Scholars Program Citation

University of Maryland

College Park, Maryland

Master of Education, Higher Education

Administration and Student Affairs, with ASJA

Mediation Certification

University of South Carolina

Columbia, South Carolina

Graduate Assistant, Office for Sexual Health \&

Violence Prevention

University of South Carolina

Columbia, South Carolina

Coordinator, Center for Leadership \& Service and

Women's Center, Biscayne Bay Campus

Florida International University

Miami, Florida

Assistant Director, Center for Leadership \& Service and Women's Center, Biscayne Bay Campus

Florida International University

Miami, Florida

Associate Director, Women's Center

Florida International University

Miami, Florida

Graduate Certificate, Women's \& Gender Studies

Florida International University

Miami, Florida

Director, Women's Center

Florida International University

Miami, Florida

Doctor of Philosophy Candidate, Higher Education Florida International University

Miami, Florida 